UNIVERSIDAD NACIONAL DE LA PLATA

FACULTAD DE CIENCIAS EXACTAS

DEPARTAMENTO: Quimica

Trabajo de Tesis Doctoral

Montmorillonitas modificadas para la retención de pesticidas poscosecha (imazalil y tiabendazol)

Tesista: Martina Gamba

Director/a: Rosa M. Torres Sánchez

Codirector/a: Gustavo Echeverria

Año: 2017 


\title{
Montmorillonitas modificadas para la retención de pesticidas poscosecha \\ (imazalil y tiabendazol)
}

\section{Martina Gamba}

\author{
Trabajo de tesis doctoral \\ para optar por el grado de \\ DOCTOR DE LA FACULTAD DE CIENCIAS EXACTAS \\ de la Universidad Nacional de La Plata
}

\author{
Directora: \\ Rosa María Torres Sánchez \\ Co-director:
}

Gustavo Echeverría

La Plata, Argentina 


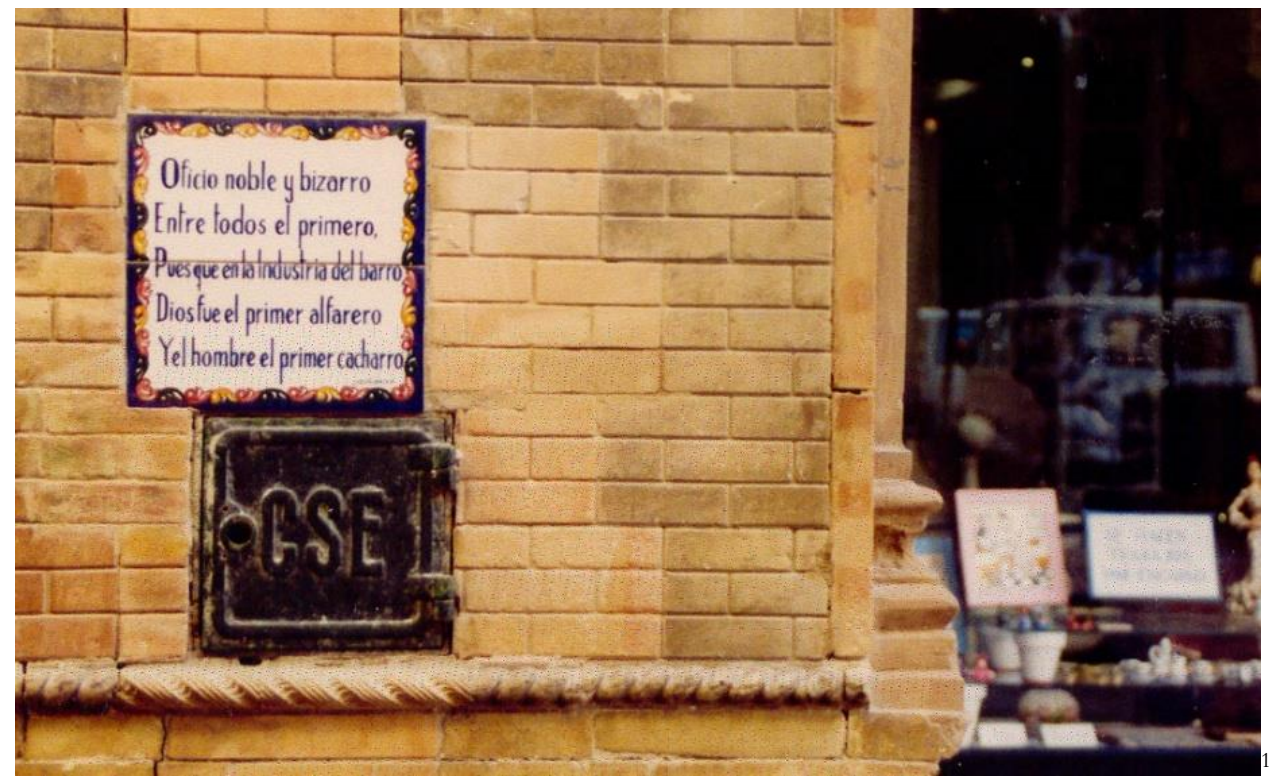

Aunque probablemente estas palabras se refieren al origen del hombre descripto en el Génesis, no sólo las religiones judeo-cristianas conciben el nacimiento de los seres humanos efectuado por dioses creadores que ejercen de alfareros. La alfarería y los mitos creadores se encuentran unidos al origen de la vida por medio de relatos mitológicos de diversas culturas antiguas, donde la idea común en todas ellas es la del Ser supremo creador que amasa la arcilla modeladora del hombre, con la tierra y el agua o incluso con su propia sangre [Sempere, 2006]. ${ }^{2}$

Quizás el origen de la vida vinculado a las arcillas, radique en el vínculo tan estrecho que milenariamente el ser humano ha tenido con estos minerales.

${ }^{1}$ Fotografía tomada por la autora en el Barrio de Triana (Sevilla, España).

${ }^{2} \mathrm{Y}$ aunque lo que la religión y las mitologías proponen acerca del nacimiento del todo pareciera estar alejado de lo que la ciencia plantea al respecto, el origen de la vida vinculado a las arcillas no lo está, o al menos, hay una discusión al respecto. El rol de las arcillas en el desarrollo de la vida fue planteado por vez primera por J. D. Bernal (1949). Las arcillas podrían haber estado involucradas en el ensamblaje de moléculas simples, primer paso en la formación de bio-polímeros como el ARN, en el caldo de cultivo que era la Tierra hace miles de millones de años atrás [Heller-Kallai et. al., 2006]. 
Los asentamientos humanos más primitivos y numerosos se concentraban principalmente en los deltas de los ríos, por la fertilidad de la tierra, por la pesca, etc. En esas zonas, la carencia de rocas consolidadas y la abundancia de arcillas de arrastre, su relativa facilidad de tratamiento, su resistencia e impermeabilidad las convirtieron en un material profusamente utilizado, principalmente para la construcción de viviendas y la fabricación de utensilios.

El trabajo como realización humana consciente más antiguo utilizando arcilla apareció hace más de 25 mil años y tiene forma de mujer: se trata de la Venus de Dolní Věstonice, una escultura de terracota encontrada en la República Checa.

Más curioso y menos difundido aún es que el soporte de la primera literatura del mundo fue hecho en arcilla: la mitología escrita más antigua, los inicios del Derecho, los primeros análisis del mundo y el universo, así como la primera Administración fueron escritas en tablillas de arcilla secadas al sol por los Babilonios en la Mesopotamia.

Milenariamente se utilizaron minerales arcillosos para remover compuestos no deseados de una matriz. Por ejemplo, las "tierras de batán" se utilizaban en el proceso de "batanado" del algodón y de la lana para extraer las grasas, aceites y otras substancias de las fibras formadas en la máquina (batán). Por lo general la arcilla utilizada era la esmectita, cuyo nombre proviene del griego smektikós: que limpia [Domínguez et al., 2013].

La utilización de las arcillas en medicina, está también indicada en textos del árabe Avicena, el anatomista griego Galeno, y el historiador Plinio, quienes atribuían una fuerza extraordinaria a la llamada Tierra de Lemnos [Wellmann, 1999].

En todos estos casos, la utilización de las arcillas fue rudimentaria y sus usos restringidos y locales. Las primeras aplicaciones elaboradas de minerales aparecieron con la fabricación de arcilla cocida, para ladrillería y fabricación de cacharros o utensilios, que posteriormente se perfeccionarían con la aparición de la industria cerámica.

Con la entrada del siglo XX y la Revolución Industrial se desarrollaron nuevos usos de estos minerales, como cargas en pinturas y detergentes, materiales de construcción más refinados, absorbentes y decolorantes, entre otros.

El conocimiento a nivel físico-químico de las arcillas permitió que en los últimos cuarenta años, con la utilización de nuevas técnicas de producción y modificaciones estructurales, se hayan diversificado sus aplicaciones. Hoy son una parte importante de la tecnología industrial, jugando diversos papeles en los procesos de fabricación y siendo constituyentes principales de diversos productos [Richerson, 1992]. 
Entre los diversos minerales arcillosos destacan por la variedad de sus aplicaciones la caolinita, las esmectitas y los minerales fibrosos: sepiolita y paligorskita. Aunque todas ellas poseen las características estructurales comunes a los filosilicatos, tales como la presencia de unidades modulares constituidas por capas tetraédricas y octaédricas ensambladas para formar láminas, presentan diferencias relativas a la organización de estas capas, y en sus composiciones químicas. Como consecuencia de estas diferencias, muestran propiedades diferentes.

En la industria del papel, la caolinita se utiliza como relleno en la hoja o en el recubrimiento de su superficie. En la industria cerámica, este mineral tiene también amplia aplicación, particularmente en la producción de piezas de pasta blanca de alta calidad tales como porcelanas, vajilla, aisladores, sanitarios, materiales refractarios y como soporte de medicamentos en píldoras y pastillas. En los plásticos, el caolín se utiliza ampliamente como carga porque mejora características tales como la obtención de superficies más suaves, acabados más atractivos y una mayor estabilidad dimensional y resistencia al ataque químico. Otra utilidad industrial del caolín, luego de su conversión a zeolitas mediante el tratamiento con hidróxidos de metales alcalinos y alcalinotérreos, es como catalizador del cracking para el refinamiento del petróleo y para remover el agua de las corrientes de gases [González López, 2000].

La paligorskita, en cosméticos actúa como una carga con propiedades tixotrópicas y también es utilizada como agente adhesivo y protector de la piel.

Las esmectitas se utilizan históricamente en las industrias petrolera, fundición, siderúrgica, veterinaria y alimentos balanceados. También, como cargas, estas arcillas pueden ser utilizadas en pinturas, esmaltes, detergentes, plásticos, cementos, adhesivos, pesticidas, etc. En particular, en alimentos balanceados de animales además de su utilización como aglomerantes, ejercen la acción de adsorbentes de bacterias y ciertas enzimas que promueven el crecimiento y la salud del animal [Magnoli et al., 2008]. Otra utilidad amplia y creciente de las bentonitas es como adsorbente de residuos domésticos. En este mercado se utilizan gránulos de esmectita cálcica y esmectita sódica pulverizada, tratadas con desodorantes, para producir camas de animales domésticos. Se aplican también como soportes de insecticidas y fungicidas y como absorbentes de grasas, aceites, aguas u otras sustancias químicas orgánicas no deseables en los suelos de fábricas, garajes, almacenes, etc.

Otros productos, basados en esmectitas funcionalizadas con distintos tratamientos, son los denominados "materiales avanzados", de potencial interés en aplicaciones tales como 
adsorbentes, catalizadores específicos, dispositivos electro-químicos, membranas selectivas y sistemas fotoeléctricos.

El hecho que la reactividad macroscópica de los minerales esté basada en sus características microscópicas hace que, en cada una de estas aplicaciones, el conocimiento de la química coloidal y la interfase sólido/líquido sea de suma importancia.

En ese sentido, el foco de esta tesis estará puesto en aportar conocimiento acerca de los diversos mecanismos físico-químicos por los cuales dos fungicidas utilizados ampliamente en la industria frutícola en nuestro país y en el mundo, interactúan con un tipo de arcilla de la familia de las esmectitas (la montmorillonita, abundante en el norte de la Patagonia) y con productos de su modificación química. Se trata de un estudio preliminar para la evaluación de su uso en el tratamiento de efluentes de la producción agrícola.

Martina Gamba

29 de Marzo de 2017 
Las arcillas son materiales que por su versatilidad y abundancia fueron milenariamente utilizados en diversas aplicaciones. Con el desarrollo del conocimiento científico, se pudo asignar a ciertas características físico-químicas sus propiedades específicas y además introducir modificaciones en su estructura con el objetivo de optimizar su aplicabilidad.

Las principales características de las montmorillonitas son su gran superficie específica y capacidad de intercambio catiónico que las convierte en excelentes adsorbentes de especies catiónicas. Para mejorar su capacidad de adsorción de especies orgánicas (neutras o aniónicas), se postula someterlas a procesos de intercambio catiónico con aminas alquílicas o metales de transición para generar nuevos sitios activos.

En Argentina, la gran actividad agrícola y, en particular, la frutícola, genera grandes volúmenes de efluentes conteniendo distintas moléculas orgánicas que en general son mezclas de fungicidas y detergentes. Antes de su disposición por vertido en aguas naturales, es recomendable tratar estos efluentes para remover posibles contaminantes. En ese sentido, la adsorción es una vía sencilla y económica y el uso de montmorillonitas naturales y modificadas una posibilidad con gran potencialidad.

El objetivo de esta tesis es aportar conocimiento acerca de los diversos mecanismos físico-químicos por los cuales dos fungicidas utilizados ampliamente en la industria frutícola en nuestro país y en el mundo (tiabendazol e imazalil), interactúan con montmorillonita (arcilla abundante en el norte de la Patagonia) y con productos de su modificación química. 
En una primera etapa se compararán las capacidades de adsorción de los fungicidas por parte de la montmorillonita natural y de organo-montmorillonitas (Capítulos 3 y 4). Luego, en una segunda etapa, se evaluará como adsorbente de estos fungicidas -tanto individuales como combinados- una montmorillonita intercambiada con cobre, un metal que también es tóxico a ciertos niveles de concentración y por lo tanto requiere ser removido de efluentes industriales y mineros (Capítulo 5). Se profundizará el estudio sobre el efecto que tiene la presencia del metal en los adsorbentes en la remoción del tiabendazol y se realizará un ensayo preliminar empleando un efluente real de plantas de empaque de frutas de pepita de la provincia de Río Negro (Capítulo 6). Para comprender los procesos se estudiarán variables como el tiempo, $\mathrm{pH}$, la concentración y se caracterizarán los materiales antes y después de los ensayos de adsorción. De esta manera se buscará identificar los sitios activos y el mecanismo involucrado. 


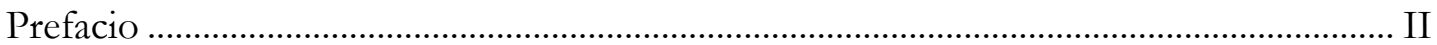

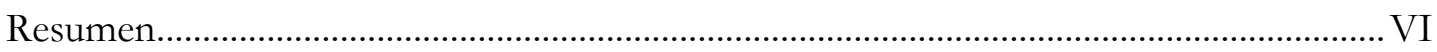

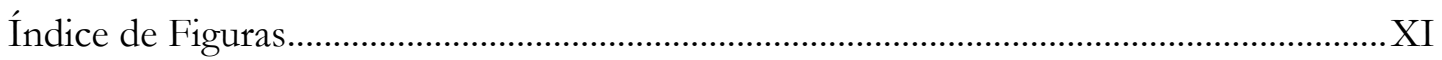

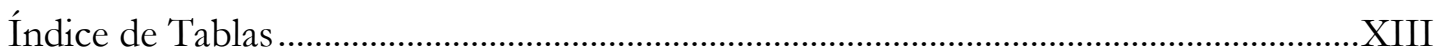

Capítulo 1. Introducción, hipótesis, objetivos y enfoque metodológico............................... 15

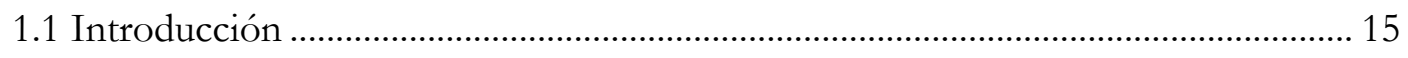

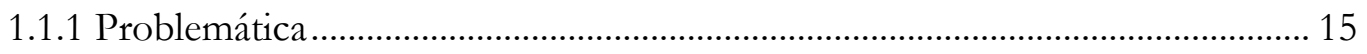

1.1.2 Fungicidas en estudio: Imazalil y Tiabendazol ................................................... 19

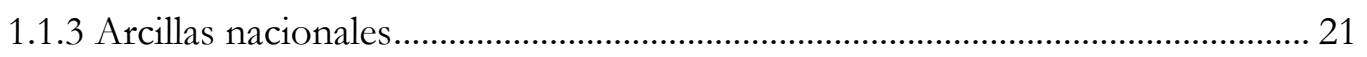

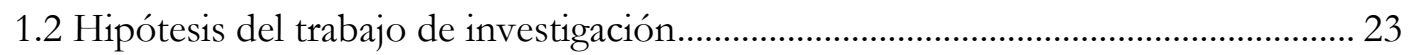

1.3 Objetivos generales de la tesis .............................................................................. 24

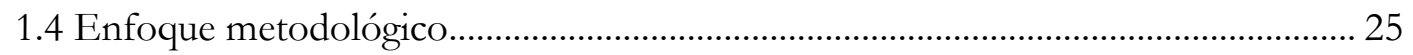

Capítulo 2. Aspectos teóricos. Propiedades físico-químicas de montmorillonitas y

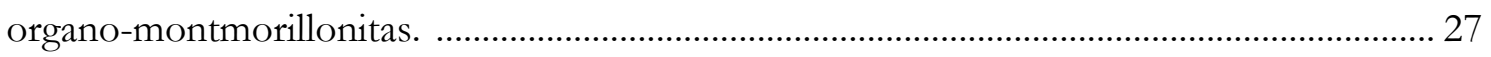

2.1 Estructura y propiedades físico-químicas de las montmorillonitas .......................... 27

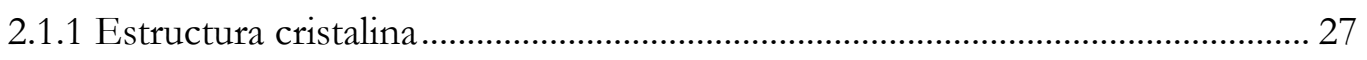

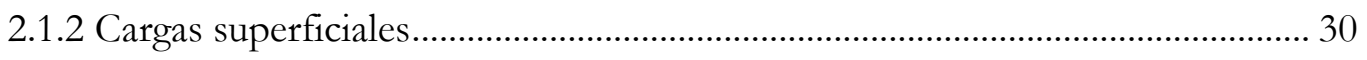

2.2.3 Capacidad de intercambio catiónico .................................................................... 32

2.2.4 Sitios activos en montmorillonitas en la interacción con materia orgánica..... 33

2.2 Obtención, estructura y propiedades físico-químicas de organo-montmorillonitas

Capítulo 3. Adsorción de imazalil en montmorillonita y organo-montmorillonitas. ...... 43

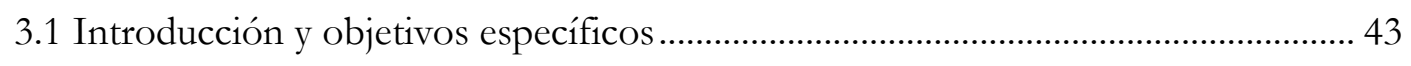

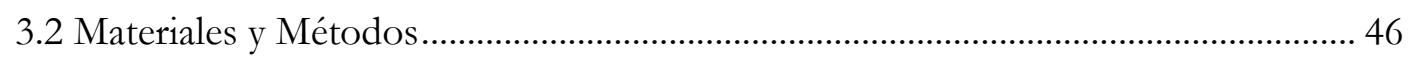

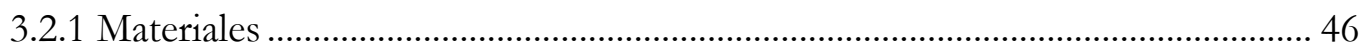

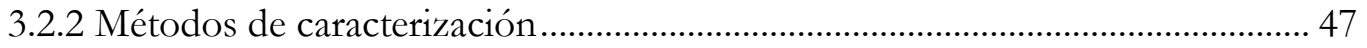




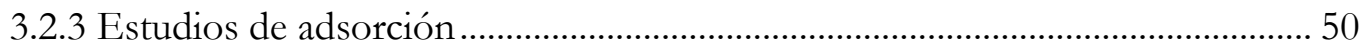

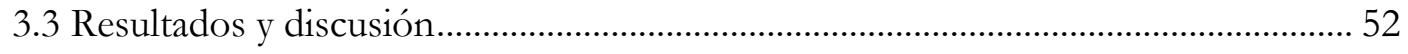

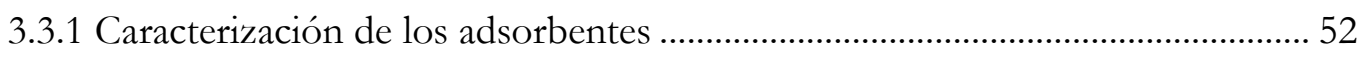

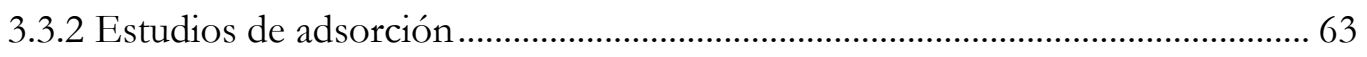

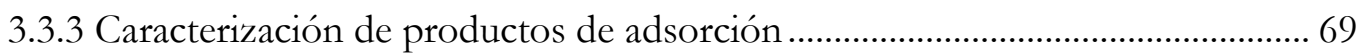

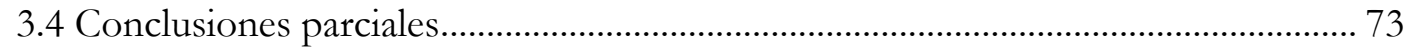

Capítulo 4. Adsorción de tiabendazol en montmorillonitas y organo-montmorillonitas .75

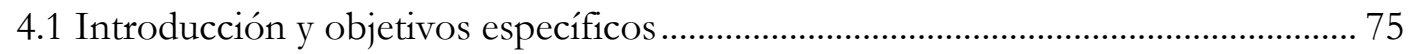

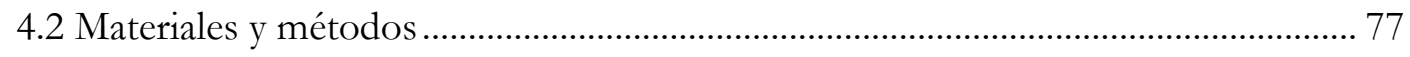

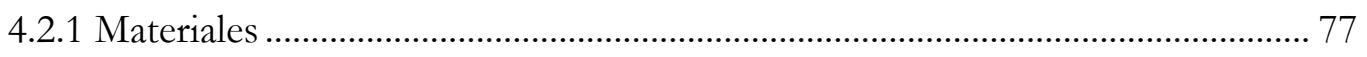

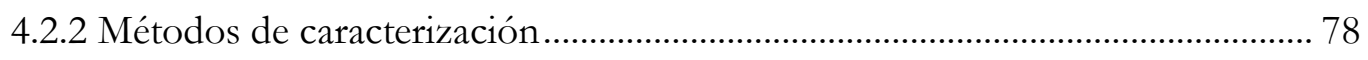

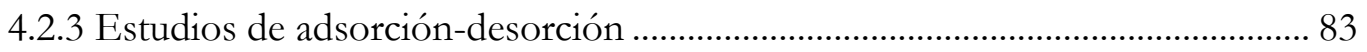

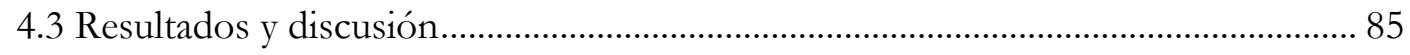

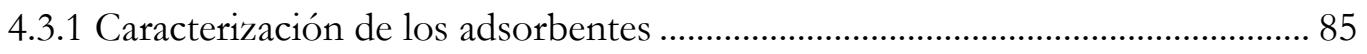

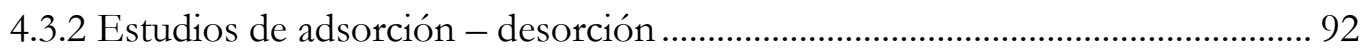

4.3.3 Caracterización de productos de adsorción ........................................................... 95

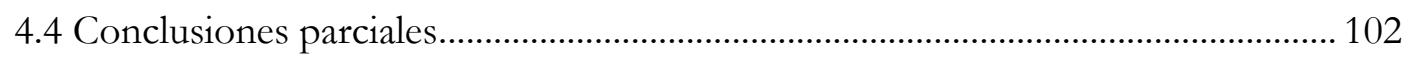

Capítulo 5. Adsorción de imazalil y tiabendazol en montmorillonitas intercambiadas con

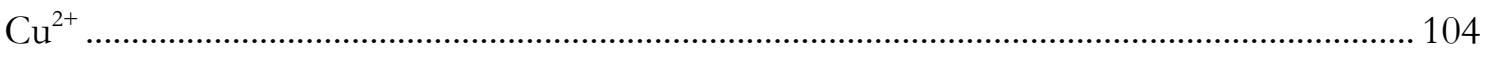

5.1 Introducción y objetivos específicos ......................................................................... 104

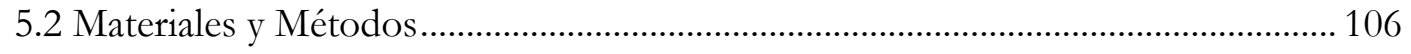

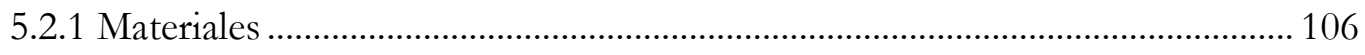

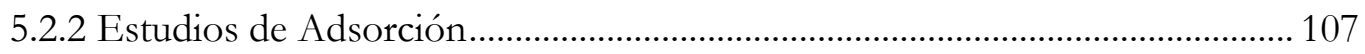

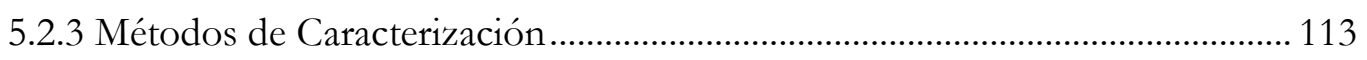

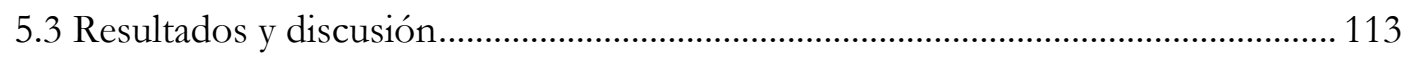

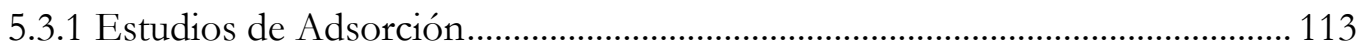

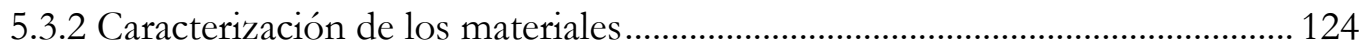


Capítulo 6. Profundización en el estudio del efecto del cobre en la estructura de la montmorillonita y de una organo-montmorillonita para la remoción de tiabendazol. Ensayos con un efluente real.

6.1 Introducción y objetivos específicos.......................................................................... 130

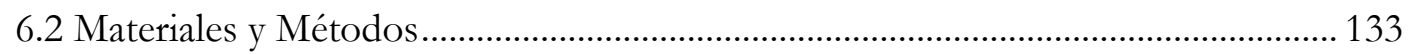

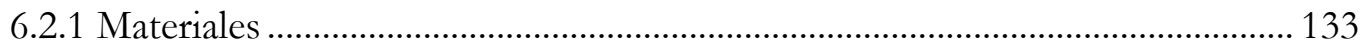

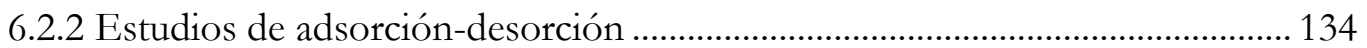

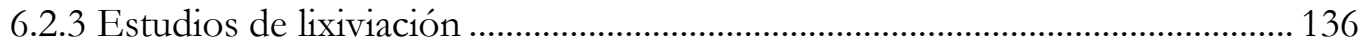

6.2.4 Métodos de caracterización ................................................................................. 136

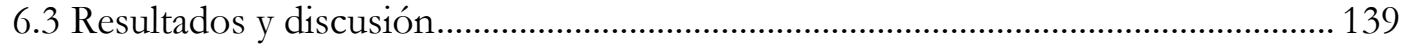

6.3.1 Estudios de adsorción .......................................................................................... 139

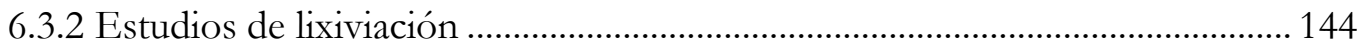

6.3.3 Caracterización de los materiales............................................................................. 146

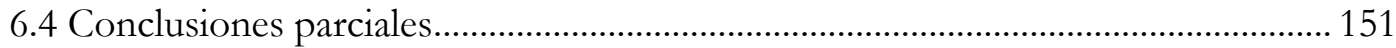

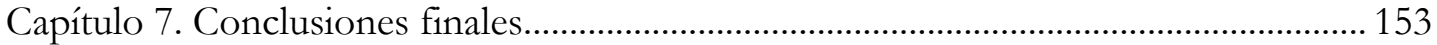

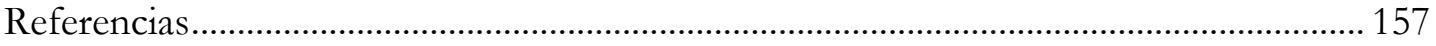

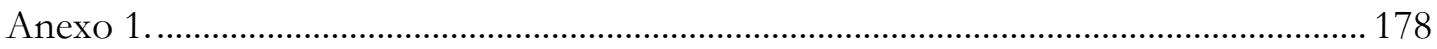

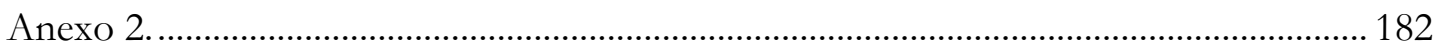


Figura 1.1 Flujo general de la fruta cuando llega al establecimiento de empaque luego de la cosecha 17

Figura 1.2 Equilibrios ácido-base del TBZ 20

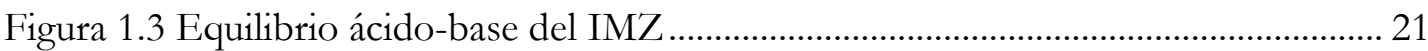

Figura 1.4 Distribución de la demanda nacional de bentonita.............................................. 22

Figura 2.1 Estructura cristalina y composición química de la montmorillonita................ 29

Figura 2.2 Equilibrios ácido-base en los sitios de bordes de la montmorillonita.............. 30

Figura 2.3 Esquema de las láminas curvadas de la montmorillonita transformándose en láminas planas con el agregado de surfactante y empaquetamiento de las cadenas alquílicas

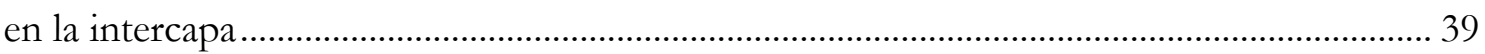

Figura 3.1 Estructura química de ODTMA............................................................................... 46

Figura 3.2 Difractogramas de Mt y OMts.............................................................................. 53

Figura 3.3 Curvas de potencial zeta vs. pH de Mt y OMts .................................................. 54

Figura 3.4 Curvas de análisis térmicos de Mt y ODTMA ………………………………...... 56

Figura 3.5 Curvas de análisis térmicos de OMts ........................................................................ 59

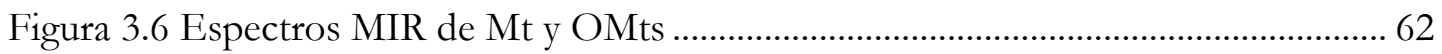

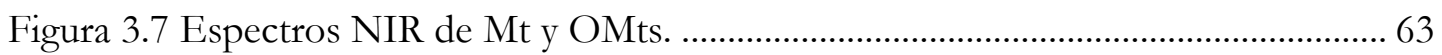

Figura 3.8 Influencia del tiempo en la adsorción de IMZ en Mt y OMt137 ..................... 63

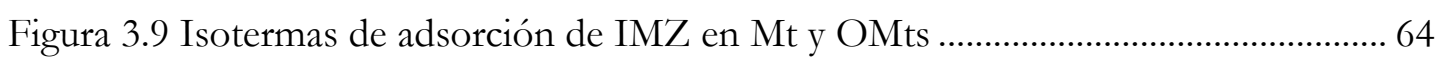

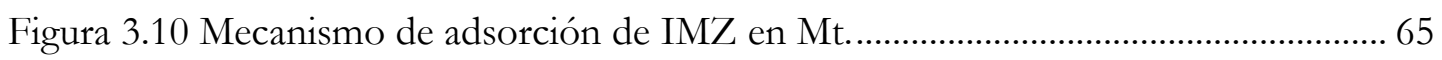

Figura 3.11 Eficiencia de remoción de IMZ vs. pH de Mt y OMts...................................... 68

Figura 3.12 Curvas de análisis térmicos de productos de adsorción de IMZ ..................... 70

Figura 3.13 Espectros MIR de IMZ y productos de adsorción de IMZ .............................. 71

Figura 3.14 Espectros NIR de IMZ y productos de adsorción de IMZ ............................. 73

Figura 4.1 Curvas de análisis térmicos de Wy y OWy........................................................... 85

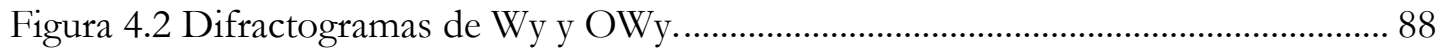

Figura 4.3 Arreglo de ODTMA en la intercapa de OWy .................................................... 89

Figura 4.4 Curvas de potencial zeta vs. $\mathrm{pH}$ de Wy y OWy...................................................... 89

Figura 4.5 Espectros de alta resolución de la banda correspondiente al electrón C1s de

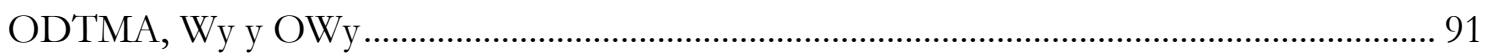

Figura 4.6 Espectro de alta resolución de la banda correspondiente al electrón N1s de

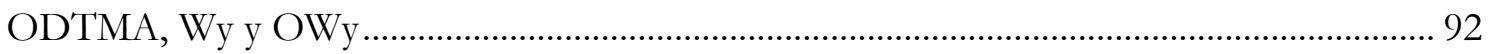


Figura 4.7 Isotermas de adsorción de TBZ en Mt, OMts, Wy y OWy .93

Figura 4.8 Difractogramas de productos de adsorción de TBZ ............................................ 95

Figura 4.9 Curvas de análisis térmicos de TBZ y TBZ-Mt .................................................... 97

Figure 4.10 Arreglo molecular de $\mathrm{TBZH}^{+}$y TBZ-Na ${ }^{+}$en la superficie de Wy ............... 100

Figura 4.11 Arreglo molecular de TBZH ${ }^{+}$y TBZ en la superficie de OWy..................... 101

Figura 5.1 Cinéticas de adsorción de IMZ y TBZ en Mt-Cu, relación sólido/solución = $1 \mathrm{~g} / \mathrm{L}$

Figura 5.2 Cinéticas de adsorción de IMZ y TBZ en Mt-Cu, relación sólido/solución = $0,1 \mathrm{~g} / \mathrm{L}$

Figura 5.3 Isotermas de adsorción de TBZ en Mt y Mt-Cu 118

Figura 5.4 Isotermas de adsorción de IMZ en Mt y Mt-Cu 119

Figura 5.5 Isotermas de adsorción múltiples de IMZ y TBZ en Mt-Cu 122

Figura 5.6 Difractogramas de Mt-Cu y productos de adsorción de TBZ e IMZ 124

Figura 5.7 Curvas de potencial zeta vs. $\mathrm{pH}$ de $\mathrm{Mt}-\mathrm{Cu}$ y productos de adsorción de TBZ e IMZ. ..... 127

Figura 5.8 Curvas de análisis térmicos de $\mathrm{Mt}-\mathrm{Cu}$ y productos de adsorción de TBZ e IMZ. 126

Figura 5.9 Mecanismos de adsorción de TBZ e IMZ en Mt-Cu. 129

Figura 6.1 Maneras de obtener un espectro de resonancia magnética. 137

Figura 6.2 Isotermas de adsorción de TBZ en Mt, Mt-Cu, OMt55, OMt55-Cu a distintas relaciones sólido/solución

Figura 6.3 Eficiencia de remoción de TBZ de Mt, Mt-Cu, OMt55 y OMt55-Cu........... 141

Figura 6.4 Eficiencia de remoción de TBZ proveniente de un efluente real de Mt, Mt$\mathrm{Cu}, \mathrm{OMt} 55$ y OMt55-Cu.

Figura 6.5 Difractogramas de OMt55, OMt55-Cu y TBZ-OMt55-Cu. ........................... 146

Figura 6.6 Curvas de análisis térmicos de OMt55-Cu y TBZ-OMt55-Cu. 147

Figura 6.7 Espectros EPR de Mt-Cu, TBZ-Mt-Cu, OMt55-Cu, TBZ-OMt55-Cu y complejo $\mathrm{Cu}-\mathrm{TBZ}$

Figura A1. Foto de suspensiones acuosas de montmorillonita y organomontmorillonitas

Figura A2. Difractograma y análisis térmicos del complejo Cu-TBZ 178

Figura A3. Foto de suspensiones acuosas de productos de adsorción de IMZ y TBZ en montmorillonita, organo-montmorillonitas con y $\sin \mathrm{Cu}^{2+}$ en su estructura 178 


\section{Indice de Tablas}

Tabla 3.1 Contenido de ODTMA y diámetro aparente de Mt y OMts. 53

Tabla 3.2 Reacciones térmicas, picos DTg y ATD y porcentajes de pérdida de masa de Mt y OMts.

Tabla 3.3 Parámetros obtenidos mediante el ajuste de los modelos de Freundlich y Langmuir a las isotermas de adsorción de IMZ en Mt y OMts..... 64

Tabla 3.4 Espaciado basal, temperatura de descomposición del contenido orgánico y potencial zeta de IMZ y productos de adsorción.

Tabla 4.1 Reacciones térmicas, picos DTg y ATD y porcentajes de pérdida de masa de Wy y OWy 86

Tabla 4.2 Composición elemental de la superficie externa determinada por XPS de ODTMA, Wy y OWy.

Tabla 4.3 Parámetros obtenidos mediante el ajuste de los modelos de Langmuir, Freundlich y Langmuir-Freundlich a las isotermas de adsorción de TBZ en Mt, OMts, Wy y OWy

Tabla 4.4 Cantidad adsorbida por unidad de masa y porcentajes de desorción de TBZ en distintos adsorbentes 94

Tabla 4.5 Reacciones térmicas, picos DTg y ATD y porcentajes de pérdida de masa de productos de adsorción de TBZ 97

Tabla 4.6 Composición elemental de la superficie externa determinada por XPS de TBZ y productos de adsorción. 98

Tabla 4.7 Potencial zeta de productos de adsorción de TBZ .99

Tabla 5.1 Condiciones y nomenclatura de cinéticas de adsorción de TBZ e IMZ en Mt$\mathrm{Cu}$. 107

Tabla 5.2 Condiciones y nomenclatura de isotermas de adsorción de TBZ e IMZ en Mt$\mathrm{Cu}$.

Tabla 5.3 Concentraciones iniciales y cantidades adsorbidas por unidad de masa de TBZ e IMZ y contenido de $\mathrm{Cu}^{2+}$ en productos de adsorción

Tabla 5.4 Parámetros obtenidos mediante el ajuste de distintos modelos matemáticos a las cinéticas de adsorción de TBZ e IMZ en Mt-Cu

Tabla 5.5 Parámetros obtenidos mediante el ajuste de los modelos de Freundlich, Langmuir y Langmuir-Freundlich a las isotermas de adsorción de TBZ en Mt-Cu 
Tabla 5.6 Parámetros obtenidos mediante el ajuste de los modelos de Freundlich, Langmuir y Langmuir-Freundlich a las isotermas de adsorción de IMZ en Mt-Cu 120

Tabla 5.7 Parámetros obtenidos mediante el ajuste de Langmuir-Freundlich a las isotermas de adsorción múltiples de TBZ e IMZ en Mt-Cu..... 122

Tabla 5.8 Reacciones térmicas, picos DTg y ATD y porcentajes de pérdida de masa de $\mathrm{Mt}-\mathrm{Cu}$ y productos de adsorción

Tabla 6.1 Parámetros obtenidos mediante el ajuste de Langmuir-Freundlich a las isotermas de adsorción de TBZ en OMt55-Cu.

Tabla 6.2 Cantidad adsorbida de TBZ por unidad de masa y porcentajes de desorción de distintos productos de adsorción.

Tabla 6.3 Contenido de $\mathrm{Cu}^{2+}$, cantidad adsorbida de TBZ por unidad de masa y porcentajes de lixiviación del metal y de TBZ de distintos productos de adsorción. 145

Tabla 6.4 Reacciones térmicas, picos DTg y ATD y porcentajes de pérdida de masa de OMt55-Cu y TBZ-OMt55-Cu 148

Tabla A1. Número de onda $\left(\mathrm{cm}^{-1}\right)$ y asignación de las bandas del espectro MIR de IMZ, Mt, OMts y productos de adsorción 179

Tabla A2. Número de onda $\left(\mathrm{cm}^{-1}\right)$ y asignación de las bandas observadas en el espectro NIR de IMZ, Mt, OMts y productos de adsorción 181 


\section{Capitulo 1. \\ Introducción, hipótesis, objetivos y enfoque metodológico.}

\subsection{Introducción}

\subsubsection{Problemática}

La producción agropecuaria en nuestro país representa aproximadamente el 32\% del Producto Bruto Interno, el $60 \%$ de las exportaciones y el $90 \%$ de los alimentos que se consumen [Pórfido, 2014]. De las 31 millones de hectáreas destinadas a esta actividad [El Cronista, 2012], más de medio millón se destina al cultivo de frutales a lo largo de todo el territorio nacional.

La producción argentina de frutas tuvo un gran impulso a partir del 2002, debido al cambio macroeconómico producido en el país a partir de la devaluación cambiaria de la moneda, que favoreció al agro en su conjunto y permitió aumentar los saldos exportables [Craviotti et al., 2010]. En 1998 las exportaciones de frutas no superaban las 864.300 Tn mientras que en el año 2014 las mismas aumentaron a casi el doble de dicho valor (1.528.053 Tn). En ese período, la producción alcanzó a superar las 7 millones de Tn repartidas principalmente en uva: 3 millones; cítricos: 2,7 millones; frutas de pepita: 1,5 millones; frutas de carozos: 400.000; olivo: 45.000; perteneciendo el resto de la producción a los cultivos tropicales, frutos secos y fruta fina. Estos cultivos representan en nuestro país una importante fuente de trabajo permanente (350.000 personas), además de mano de obra temporaria (golondrina) y la empleada en los procesos de producción (vino, jugos, aceite, 
etc.) y la participación de 20.000 productores. Asimismo, en muchas regiones, estos cultivos sostienen las economías provinciales [Idigoras, 2014].

El 85\% de la producción de manzana y el $75 \%$ de la de pera se concentran en el Alto Valle (Río Negro y Neuquén) y en el Valle Medio (Río Negro). El resto se genera en el Valle de Uco (Mendoza), 25 de Mayo (La Pampa) y el Valle del Tulum (San Juan), entre otros puntos [CAFI, 2014]. Por otro lado, la producción de naranjas, mandarinas, pomelos y limones -que le ha permitido al país alcanzar el primer lugar como productor de limón y exportador de jugo de limón y ubicarse en la novena posición entre los productores mundiales de cítricos- abarca 131.000 hectáreas situadas principalmente en el noroeste y litoral argentinos (provincias de Jujuy, Salta, Tucumán, Catamarca, Misiones, Corrientes y Entre Ríos) [SENASA, 2014].

La cadena productiva tanto de frutas cítricas como de pepitas (peras y manzanas) consiste en las siguientes etapas: siembra y cultivo, cosecha, poscosecha y empaque.

Luego de la cosecha, la fruta es transportada en cajones (bin) hasta las plantas empacadoras, donde se realizan las labores de poscosecha y empaque. Cuando la fruta llega a la planta, puede ser procesada directamente "en caliente", y/o almacenada en cámaras frigoríficas para su posterior procesamiento (previo lavado y tratamiento con productos fitosanitarios) (Figura 1.1). Ya sea embalada o en bines, el almacenamiento frigorífico puede prolongarse desde unos pocos días hasta un año.

El proceso en la línea de empaque (Figura 1.1) se inicia en el volcado, que se realiza tanto en agua conteniendo hipoclorito de sodio $(200 \mathrm{mg} / \mathrm{L})$ como en seco. En la actualidad, el sistema más utilizado es la hidro-inmersión, la cual minimiza los golpes y permite trabajar con volúmenes de fruta elevados, aumentando el rendimiento global del resto del proceso. En la etapa de preclasificación se realiza una primera selección, para descartar frutos con defectos importantes de calidad. A continuación, en el módulo de lavado la fruta se lava con detergente, se enjuaga con agua y se trata con productos fitosanitarios. En el caso particular de las manzanas, en este sector se realiza también el presecado y la aplicación de cera artificial (encerado). En el sector de secado se aplica aire forzado con el objetivo de acelerar el secado de la fruta, para que ingrese a las siguientes secciones en óptimas condiciones para su manipuleo y empaque. Durante la operación de clasificación se selecciona la fruta para su comercialización en diferentes grados de calidad. La fruta seleccionada por calidad se separa en la siguiente sección por su tamaño, mediante la determinación del volumen o del peso. Finalmente, la fruta seleccionada según su grado de calidad y tamaño, es embalada en los diferentes tipos de envases comerciales, enfriada y 
almacenada normalmente entre -1 y $0{ }^{\circ} \mathrm{C}$ en cámaras frigoríficas hasta su despacho final [Colodner, 2011].

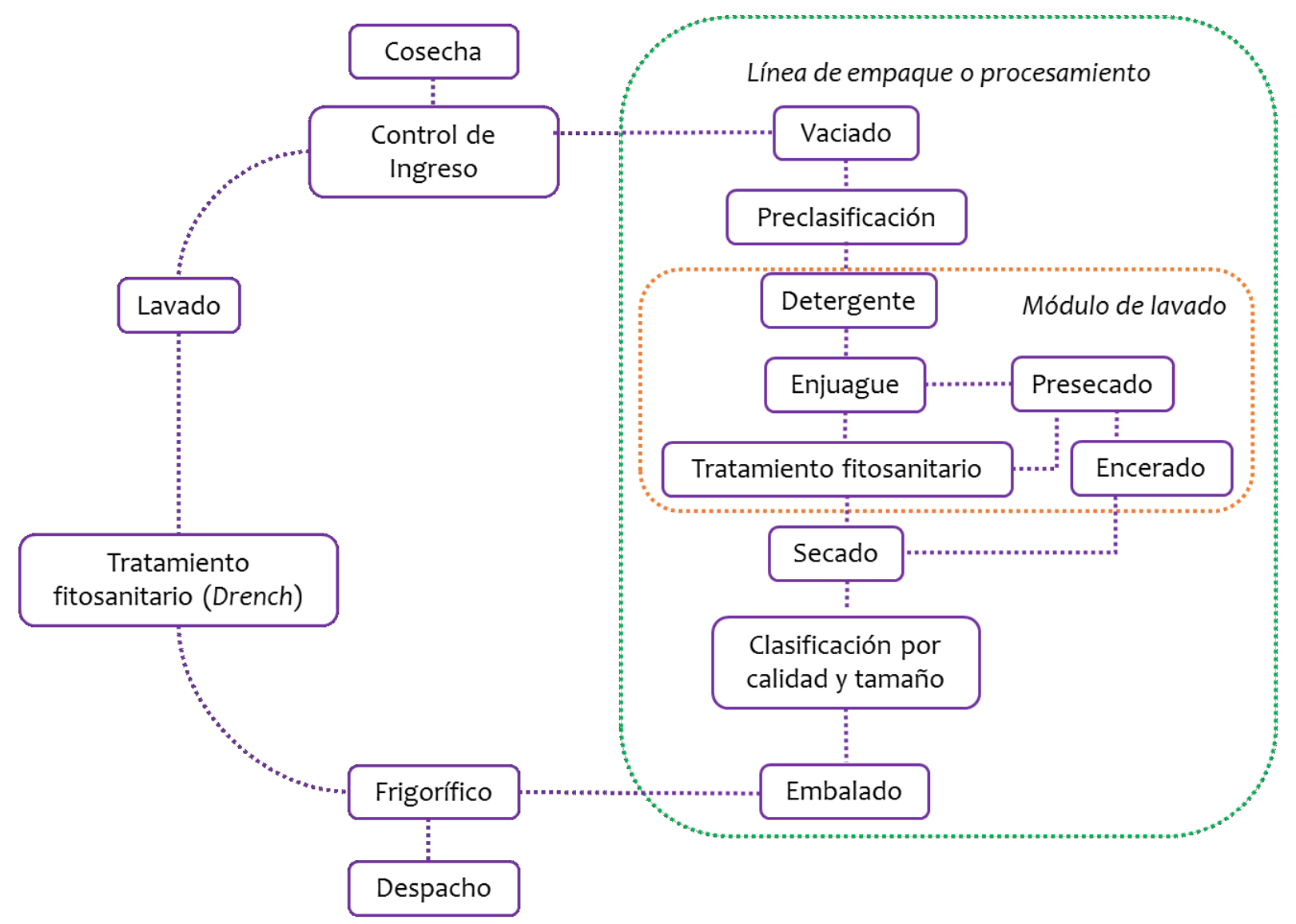

Figura 1.1 Flujo general de la fruta cuando llega al establecimiento de empaque luego de la cosecha. Fuente: [Colodner, 2011].

En las plantas empacadoras, hay por lo tanto, dos instancias donde puede realizarse la aplicación de productos fitosanitarios: mediante drench antes del ingreso a la cámara frigorífica o durante el procesamiento en la línea de empaque (Figura 1.1.).

En el drench la fruta cosechada y dispuesta dentro de los cajones bin se somete a duchas con productos antiescaldantes y fungicidas para el control de podredumbres durante el almacenamiento en la cámara frigorífica. La ducha puede aplicarse a un camión completo, a cargas de bines de un remolque con fruta o a unos pocos bines por vez circulando a través de una cinta transportadora [Janisiewicz et al., 2005]. Este método permite tratar una gran cantidad de fruta en un período de tiempo relativamente breve. Entre sus principales desventajas radica el hecho de que requiere un volumen elevado de caldo que, aunque sea recirculado para tratar bines adicionales, su disposición final representa un considerable problema [Rosenberg, 2010]. 
En la línea de empaque, la aplicación de productos fitosanitarios se realiza en el módulo de lavado utilizando sistemas de duchas o de pulverización con boquillas. Este último es el sistema de aplicación actualmente más utilizado en la región del Alto Valle de Río Negro [Colodner, 2011].

Todas estas tecnologías poscosecha tienen por objetivo disminuir las pérdidas que existen entre la cosecha y el consumo. Pequeñas mermas en la calidad durante el procesamiento y almacenamiento pueden representar importantes pérdidas económicas, ya que es en esta etapa donde se acumulan los mayores costos de producción [Colodner, 2011].

Las aguas residuales conteniendo restos de fungicidas y detergentes provenientes tanto del módulo de lavado como del drench, actualmente van a parar a piletas de decantación descubiertas donde permanecen un tiempo determinado. Su disposición final representa un problema considerable [Rosenberg, 2010]; puede transformarse en una fuente puntual de contaminación de los recursos naturales de agua [Hameed et al., 2009] y constituir un deterioro persistente, amenazante y perjudicial para la supervivencia de la flora y la fauna de los ecosistemas acuáticos [Foo et al., 2010]. En ese sentido, el Departamento Provincial de Aguas de Río Negro ha impuesto importantes restricciones legales a los establecimientos de empaque de frutas respecto a la disposición final de estos efluentes [Resolución 335/02].

Es importante destacar que el Consejo Federal de Ciencia y Tecnología (COFECyT) dependiente del Ministerio de Ciencia, Tecnología e Innovación Productiva de la Nación definió como una de las "debilidades cuya superación implica un desafío científicotecnológico" la existencia de "escasas investigaciones sobre el impacto de residuos de plaguicidas en la fruta fresca y en el medio ambiente” [COFECýT, 2008]. Más aún, en el informe del Ministerio de Salud de la Nación, Pórfido [2014] declara que "existe déficit en el diagnóstico y notificación de intoxicaciones por plaguicidas en nuestro país y, cuando esta información existe, resulta difícil de comparar pues su recolección se realiza de modo no uniforme”. A modo de ejemplo, en una investigación epidemiológica realizada en la provincia de Jujuy, Altamirano et al. [2004] hallaron que el $25 \%$ de la población de trabajadores rurales estudiada, tuvo al menos una intoxicación aguda generada por plaguicidas y aproximadamente la mitad de ellos (49,5\%) no acudieron para su tratamiento a servicios de salud. Esta investigación introduce como problema sanitario el subregistro de las intoxicaciones, panorama que dificulta la implementación de planes preventivos, una situación que se extiende a nivel nacional [Souza Casadinho, 2008a, 2009]. 
Ningún segmento de la población está completamente protegido de la exposición a los plaguicidas, en un país donde la actividad agrícola es una de las principales economías. Si bien para la gran masa poblacional la exposición es baja, los efectos tóxicos resultantes de una exposición prolongada suelen ser difíciles de distinguir, y en general, pasan inadvertidos. Los efectos biológicos resultantes de estos bajos niveles de exposición son, en general, difíciles de ser asociados a un plaguicida en particular y, muchas veces, la sintomatología que presentan enmascara la verdadera causa de la afectación, confundiéndola con un sinnúmero de otras causas probables.

Por todo lo antes expuesto, resulta fundamental que el Estado y las empresas arbitren los medios necesarios para reducir los riesgos de contaminación del suelo, el aire y el agua con plaguicidas y así evitar la exposición de los seres humanos, flora y fauna a compuestos potencialmente tóxicos.

\subsubsection{Fungicidas en estudio: Imazalily Tiabendazol}

Entre los fungicidas poscosecha más utilizados en las plantas empacadoras de cítricos y frutas de pepita se encuentran el Tiabendazol (TBZ) y el Imazalil (IMZ).

El TBZ, al igual que otros fungicidas derivados de bencimidazoles, apareció en el mercado a partir de la década del 1960 [Bovi Mitre et al., 2004]. Es un fungicida sistémico que actúa inhibiendo la mitosis celular [INTA, 2016]. Actualmente, lo comercializa la empresa Sygenta Agro S.A., en forma de suspensión concentrada bajo el nombre de TECTO ${ }^{\circledR} 50$ SC, con una concentración de principio activo de 48,5\% p/v, aspecto lechoso y muy baja solubilidad. Se aplica en forma de suspensión para combatir, en cítricos, el moho azul, moho verde, melanosis y podredumbre del extremo del pedúnculo; en manzanas, sarna del manzano y oídio del manzano; en peras, moho azul, moho gris y antracnosis según la hoja de seguridad del producto comercial. En la etiqueta del mismo se recomienda utilizar 100-1000 $\mathrm{cm}^{3} / \mathrm{hL}$ de agua en el tratamiento fitosanitario de cítricos; 34$67 \mathrm{~cm}^{3} / \mathrm{hL}$ en el tratamiento de manzanas y $100-170 \mathrm{~cm}^{3} / \mathrm{hL}$ en el de peras.

Por su parte, el IMZ es fungicida sistémico, derivado del imidazol, que fue introducido por la Janssen Pharmacentica en 1972 [Bovi Mitre et al., 2004]. Actúa inhibiendo la síntesis del ergosterol, altera la función de la membrana e inhibe la esporulación [INTA, 2016]. Actualmente lo comercializa la empresa Magan Argentina S.A. en forma de concentrado emulsionable, bajo el nombre de XEDREL 50 ( , con una concentración de principio activo de 47,2\% p/p. El concentrado presenta una coloración variable de amarillenta a parda, muy baja solubilidad en agua y alta solubilidad en solventes orgánicos. Se aplica en 
forma de suspensión. La etiqueta del producto comercial recomienda utilizar dosis de 50 $200 \mathrm{~cm}^{3} / \mathrm{hL}$ de agua para combatir el moho azul, moho verde, podredumbre de los frutos, podredumbre de los citrus y podredumbre interna que afectan a los cítricos y 57-100 $\mathrm{cm}^{3} / \mathrm{hL}$ de agua para combatir el moho azul y podredumbre gris de los frutales de pepita.

Aunque la toxicidad del TBZ en humanos es baja, se ha demostrado que provoca intoxicación en los animales debido a trastornos en hígado e intestino [Tada et al., 1992; 2001; Price et al., 2004; Groh et al., 2012]. Por otro lado, el IMZ es clasificado como "Clase III - Poco peligroso" por la Cámara de la Industria Argentina de Fertilizantes y Agroquímicos [CIAFA, Manual Fitosanitario] y como "probablemente carcinógeno en humanos" por la Agencia de Protección Ambiental de EE.UU. [Masiá et al., 2014]. En nuestro país se han encontrado en limones restos de IMZ excediendo en un 10\% los límites máximos de residuos (LMR) fijados por el Servicio Nacional de Sanidad y Calidad Agroalimentaria (SENASA) [Hjorth et al., 2011]. Sin embargo, a nivel nacional no tenemos datos de toxicidad manifiesta en seres humanos con estos fungicidas.

El cuanto a las propiedades físico-químicas, el TBZ (de nombre IUPAC 2-(tiazol-4-il) bencimidazol) es un compuesto orgánico cuya estructura está conformada por dos anillos heterocíclicos aromáticos unidos entre sí, uno de bencimidazol y uno de tiazol (Figura 1.2). A temperatura ambiente es un sólido blanco cristalino, presenta punto de fusión de $298^{\circ} \mathrm{C}$ y masa molecular de 201,3 g/mol. El TBZ es poco soluble en solventes orgánicos no polares como el heptano y es soluble en solventes orgánicos polares como el metanol y la acetona. En agua, su solubilidad es fuertemente dependiente del $\mathrm{pH}$ : a $\mathrm{pH} 4$ presenta una solubilidad de $160 \mathrm{mg} / \mathrm{L}$ mientras que a $7<\mathrm{pH}<10$ ésta disminuye a $30 \mathrm{mg} / \mathrm{L}$ (a $20{ }^{\circ} \mathrm{C}$ ). La variación de la solubilidad con el $\mathrm{pH}$, está directamente relacionada con los equilibrios ácido-base que el compuesto experimenta en agua [European Commission, 2001] (Figura $1.2)$.

$\left(\mathrm{TBZH}_{2}{ }^{2+}\right)$<smiles></smiles>

$\mathrm{H}$<smiles>c1ccc2[nH+]c(-c3cscn3)[nH]c2c1</smiles>

$\mathrm{H}$

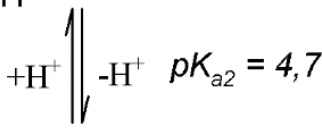

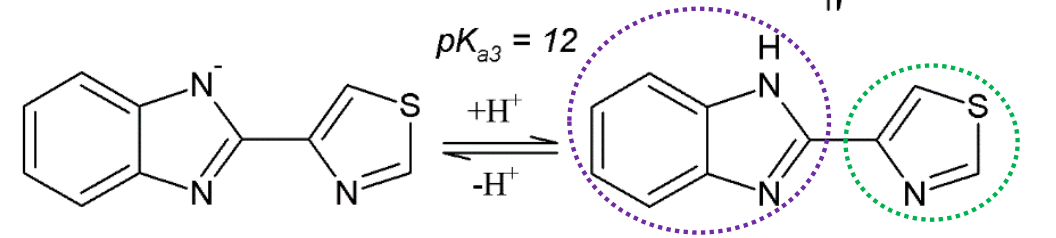

(TBZ)

Figura 1.2 Equilibrios ácido-base de TBZ. ( $\cdots)$ Anillo bencimidazol, ( $\cdots$ ) anillo tiazol. 
Como puede observarse en la Figura 1.2, las diferentes especies químicas asociadas a éste se generan por la protonación-deprotonación de los átomos de nitrógeno presentes en la molécula [Aharonson y Kafkafi, 1975a; European Commission, 2001]. Las especies más importantes en las condiciones de trabajo $(5<\mathrm{pH}<7$ ) son la neutra (TBZ) y la monoprotonada $\left(\mathrm{TBZH}^{+}\right)$.

El IMZ (de nombre IUPAC (RS)-1-( $\beta$-aliloxy-2,4-diclorofeniletil) imidazol) es una base débil con $\mathrm{p} K_{a}=6,49$. Su basicidad radica en la presencia del anillo imidazol, con un átomo de nitrógeno disponible para protonarse (Figura 1.3). A temperatura ambiente es un sólido blanco cristalino, presenta punto de fusión a $51,5^{\circ} \mathrm{C}$ y masa molecular de $297,2 \mathrm{~g} / \mathrm{mol}$. Se trata de un compuesto muy soluble en solventes orgánicos y solubilidad en agua de 184 $\mathrm{mg} / \mathrm{L}$.

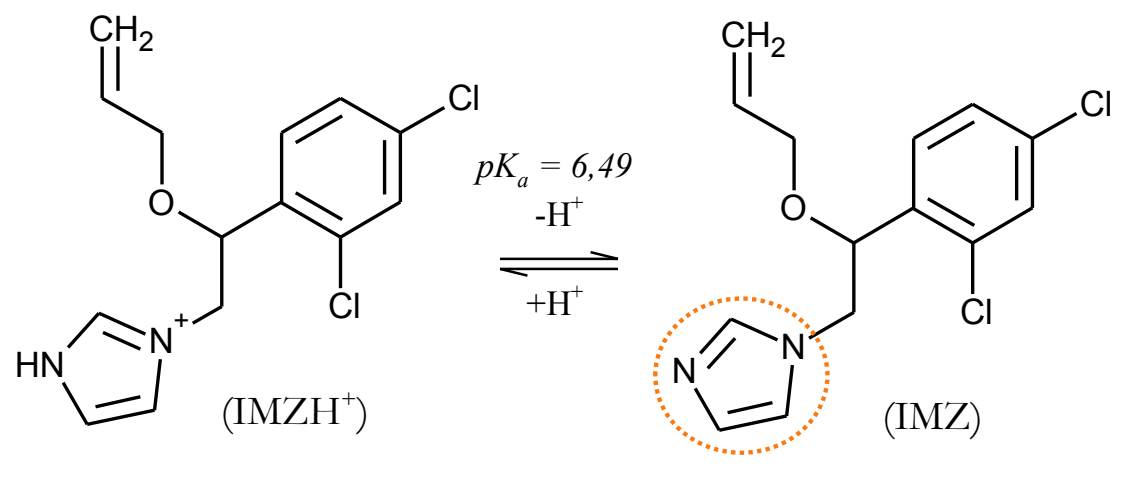

Figura 1.3 Equilibrio ácido-base de IMZ. (․) Anillo imidazol.

\subsubsection{Arcillas nacionales}

En nuestro país encontramos arcillas en diversos yacimientos de las provincias de Buenos Aires, Córdoba, Chubut, Entre Ríos, Jujuy, La Rioja, Mendoza, Misiones, Neuquén, Río Negro, Salta, San Juan, San Luis, Santiago del Estero y Tucumán. Según el tipo de arcillas, sus propiedades y calidad, se emplean en la industria del cemento portland, industria de la cerámica roja o blanca, fabricación de revestimientos cerámicos para piso o de ladrillos refractarios, entre otras aplicaciones [Zhou et al., 2014; 2016].

Los depósitos más importantes de bentonita (arcilla rica en esmectita) se ubican en el ámbito de la cuenca sedimentaria Neuquina (que abarca parte de las provincias de Neuquén, Río Negro y La Pampa). La producción de Río Negro representa casi la mitad de la producción de bentonita a nivel nacional, mientras que La Pampa ocupa el segundo lugar generando el 20\% de la producción nacional [SEGEMAR, 2004]. 
Según el censo del 2009, la producción total de bentonita en el país fue de 175.886 Tn (en bruto) [INDEC, 2009]. La producción nacional de bentonita atiende satisfactoriamente los requerimientos de la demanda interna, tanto en cantidad como en calidad. Además Argentina exporta bentonita a más de una docena de países, tanto de Sudamérica (Brasil, Chile, Bolivia, Uruguay, Paraguay, Perú, Venezuela y Colombia) como de Centroamérica (Jamaica, Guatemala), Europa (Italia y Suiza) e incluso Asia (Taiwan, Tailandia). Sin embargo, es Brasil el principal destino ( $90 \%$ de las exportaciones) ya que consume entre 40.000 y 50.000 Tn de bentonita argentina por año.

La estructura de la demanda nacional de bentonita se distribuye según el gráfico mostrado en la Figura 1.4. Excepto las industrias cerámicas que consumen el mineral en bruto tal cual es extraído de la mina, normalmente previo al despacho, la bentonita es molida y clasificada por su granulometría en función de su destino final, variando su tamaño entre $840 \mu \mathrm{m}$ en alimentos balanceados hasta $37 \mu \mathrm{m}$ en cosmética.

\begin{tabular}{|c|c|c|}
\hline ه Lodos para perforación & & $60 \%$ \\
\hline Arenas de fundición & & $14 \%$ \\
\hline a Fertilizantes & & $0,5 \%$ \\
\hline ه Alimentos balanceados & & $6 \%$ \\
\hline Industria de Pinturas & & $0,9 \%$ \\
\hline $\begin{array}{c}\text { Industria Médica } \\
\text { Cosmética }\end{array}$ & $\mathrm{y}$ & $0,1 \%$ \\
\hline Industria Vitivinicola & & $0,5 \%$ \\
\hline \Industria de Cerámica & & $12 \%$ \\
\hline$\underset{\text { grasas }}{\square \text { Industria de Aceites }}$ & $y$ & $4 \%$ \\
\hline Otros Usos & & $2 \%$ \\
\hline
\end{tabular}

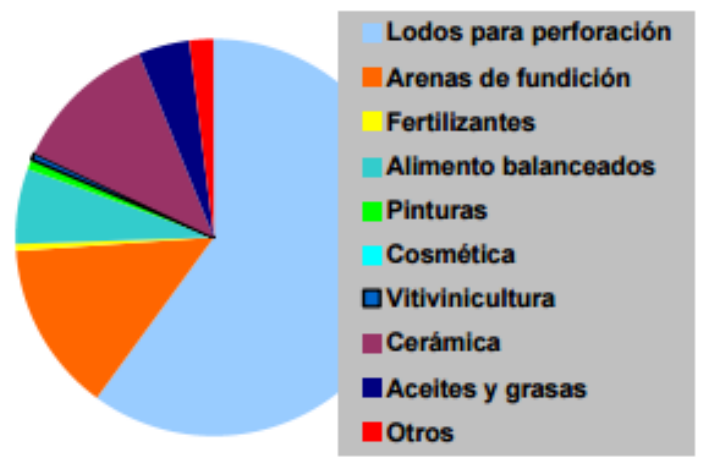

Figura 1.4 Distribución de la demanda nacional de bentonita. Fuente: [Dirección de Minería de San Juan].

En Río Negro, la mayor parte de producción se destina a lodos de perforación utilizados por empresas que operan dicho rubro en Neuquén, Río Negro y Chubut y el resto es exportado al mercado extra-regional de la industria de fundición. En la provincia de San Juan, el mineral extraído del yacimiento más importante se procesa en la Planta del Parque Industrial de Mendoza y su destino es la industria del petróleo, fundición y clarificantes. En Mendoza, con plantas de trituración y molienda, el destino de la producción es la industria petrolera, vitivinícola, clarificante y de fundiciones, tanto de Argentina como de Chile.

La importancia de los yacimientos de bentonita de la cuenca Neuquina radica en que están formados casi exclusivamente por montmorillonitas, acompañadas de impurezas (feldespatos, cuarzo, yeso, trazas de vidrio, zeolitas, etc.). Estos yacimientos se explotan en 
los alrededores del Lago Pellegrini (Río Negro), en el Departamento Puelén (La Pampa) y en la zona de Barda Negra y Cerro Bandera (Neuquén). El área de mayor producción está ubicada en el noroeste de la provincia de Río Negro, cercana a los municipios de Cinco Saltos, Barda del Medio y Comandante Cordero. Los afloramientos circundan al Lago Pellegrini -una cuenca artificial formada por las aguas del Río Neuquén- por el sur, este y noroeste [Iñíguez, 1972].

Excepto los procesos de clasificación por granulometría y molienda, actualmente son pocas las empresas que a nivel industrial agreguen valor a las bentonitas como podría ser la producción de bentonitas orgánicas o modificación química de otra índole.

\subsection{Hipótesis del trabajo de investigación}

El trabajo de investigación de esta tesis se sustentó en las siguientes hipótesis y antecedentes:

1) La adsorción es un proceso reconocido como eficiente en el tratamiento de aguas residuales [Castillo et al., 2000]. Ha sido ampliamente estudiado para la eliminación de contaminantes de efluentes acuosos mediante el uso de varios tipos de materiales porosos, por lo cual podría ser utilizado para la remoción de fungicidas poscosecha de la producción frutícola.

2) Los minerales de arcilla como la montmorillonita presentan propiedades que las postulan como buenos adsorbentes [Jaber et al., 2013]:

(i) presentan carga negativa externa (lo que las hace particularmente útiles para la eliminación de compuestos catiónicos de agua);

(ii) presentan gran área superficial (lo cual les permite anclar y concentrar una alta cantidad de moléculas e iones en su superficie); y

(iii) presentan la capacidad de aceptar diversos grupos funcionales (por ejemplo mediante reacciones de intercambio catiónico, pilareado, etc.) que modifican sus características ampliando sus aplicaciones.

Estas propiedades sumadas al hecho de que son materiales abundantes y económicos en nuestro país, las hace plausibles de ser consideradas para el tratamiento de efluentes de la producción frutícola, donde la adsorción sea el proceso fundamental.

3) La desventaja que presenta este mineral y las arcillas naturales en general es que su tamaño de partícula sumado a su hidrofilicidad -originada por la presencia de cationes interlaminares hidratados- provocan que su separación del agua sea dificultosa y que para tal fin deban usarse técnicas de floculación o centrifugación [Roca Jalil et al., 2014]. En ese 
sentido, las montmorillonitas intercambiadas con cationes orgánicos (organomontmorillonitas) presentan una ventaja, ya que el aumento del tamaño de partícula de alrededor de siete veces [Gamba et al., 2015] y su hidrofobicidad promueven una buena separación de la suspensión. Esto, sumado a los reportes de aumento de la capacidad de adsorción de montmorillonitas intercaladas con aminas alquílicas respecto de la montmorillonita natural [Fatimah y Huda, 2013], plantea la posibilidad de éxito de su uso para la adsorción de los fungicidas tiabendazol (TBZ) e imazalil (IMZ).

4) Los compuestos derivados del bencimidazol y del imidazol forman complejos de coordinación estables con iones metálicos de transición [Rong et al., 1991; Grevy et al., 2002; Ennajih et al., 2012; Holešova et al., 2009], lo que sugiere que la incorporación de un metal de transición en la estructura de la arcilla podría mejorar aún más su rendimiento en la adsorción de TBZ e IMZ. Esto además permitiría utilizar como materiales adsorbentes los sólidos resultantes de la remoción de contaminantes metálicos provenientes de efluentes mineros, industriales, etc.

5) Las propiedades macroscópicas guardan directa relación con la reactividad microscópica. Por lo tanto, un conocimiento profundo y detallado de las interacciones entre el adsorbato y el adsorbente, así como de los mecanismos que gobiernan la adsorción permitirían optimizar el proceso y el diseño del material a utilizar.

\subsection{Objetivos generales de la tesis}

1) Generar conocimiento que pueda aportar a la cadena de valor agregado de bentonitas nacionales.

2) Obtener y caracterizar montmorillonitas modificadas mediante reacciones de intercambio iónico.

3) Evaluar la capacidad de los materiales obtenidos como adsorbentes de dos fungicidas ampliamente utilizados en la producción frutícola.

4) Hacer un estudio detallado de los cambios estructurales generados en los adsorbentes por la adsorción de los fungicidas.

5) Identificar sitios activos involucrados, fuerzas de interacción y mecanismos de adsorción en todos los casos.

6) Generar conocimiento en torno al potencial uso de montmorillonitas nacionales y sus derivados en el tratamiento de efluentes de producción frutícola. 


\subsection{Enfoque metodológico}

Sintéticamente, la metodología de estudio consistió, en una primer etapa, en la obtención de diferentes organo-montmorillonitas a través de reacciones de intercambio catiónico, seguida de su caracterización empleando técnicas que permitieron evidenciar los cambios ocurridos en sus superficies reactivas: difracción de rayos X (DRX) para la superficie interna; medidas de potencial zeta para evidenciar cambios en la carga eléctrica de la superficie externa y de borde; análisis térmicos diferenciales y termogravimetría (ATD/Tg) para evidenciar cambios a nivel general (bulk); además de técnicas complementarias que se utilizaron en casos particulares como simulaciones computacionales, espectroscopía infrarroja, resonancia paramagnética electrónica, etc.

Una vez caracterizados los materiales, se procedió en su evaluación como adsorbentes de los fungicidas seleccionados. Para ello se realizaron experimentos en batch, donde se modificaron las variables tiempo, $\mathrm{pH}$, relación sólido/solución, concentración inicial del fungicida en solución. Se utilizaron modelos matemáticos para ajustar los datos experimentales y así comparar el desempeño de los distintos adsorbentes.

El paso final consistió en la caracterización de los productos de adsorción, esto es, del sólido recuperado tras las distintas adsorciones. La caracterización se realizó empleando las mismas técnicas utilizadas previamente para el material de partida.

En una segunda etapa del estudio, se obtuvieron materiales derivados de la montmorillonita mediante su intercambio catiónico con un metal de transición, el cobre, el cual es también contaminante -a partir de ciertos valores de concentración- y está presente en aguas de escorrentía de las actividades mineras e industriales. El procedimiento seguido fue similar al detallado previamente: se caracterizó el material de partida, luego se realizaron experimentos de adsorción variando los parámetros antes indicados; por último, se realizó la caracterización de los productos obtenidos luego de la adsorción.

La estructura cristalina y propiedades físico-químicas de las montmorillonitas, sus sitios activos y las posibles fuerzas de interacción con la materia orgánica, así como los cambios introducidos en los mismos al intercalarla con aminas orgánicas y los fundamentos de las técnicas que se utilizaron para evidenciarlos (DRX, potencial zeta y ATD/Tg) se describen en el Capítulo 2, ya que son bases teóricas necesarias para entender los resultados experimentales obtenidos. Además de estas técnicas, se recurrió a análisis específicos para obtener información adicional en cada sistema de estudio. Por ejemplo, en el Capítulo 3 para dilucidar los cambios en el entorno químico de los hidroxilos de la capa siloxánica de la montmorillonita y de las organo-montmorillonitas tras la adsorción del imazalil, se utilizó 
espectroscopia de infrarrojo medio y cercano (MIR y NIR, respectivamente); en el Capítulo 4, para conocer en detalle las energías de interacción entre el tiabendazol y la montmorillonita y organo-montmorillonitas se emplearon técnicas de mecánica molecular y espectroscopía de emisión de fotoelectrones (XPS) para conocer la composición química de la superficie externa; y en el Capítulo 6, para comprender el estado de oxidación y los cambios en el entorno electrónico del cobre en las muestras intercambiadas con este metal, así como de los productos de adsorción, se empleó resonancia paramagnética electrónica (EPR).

La caracterización de los materiales previos y posteriores a los ensayos de adsorción permitió evidenciar cambios estructurales promovidos en el adsorbente por la interacción con el fungicida correspondiente, lo cual posibilitó inferir los sitios activos involucrados. Así mismo, otras variables como el $\mathrm{pH}$, fueron medidas antes y después de la adsorción para dilucidar la existencia o no de reacciones ácido-base en el proceso.

En cada capítulo se resumen conclusiones parciales, las cuales aportan a la conclusión general de este trabajo de tesis. 


\section{Capitulo 2. \\ Aspectos teóricos. Propiedades físico-químicas de montmorillonitas y organo-montmorillonitas.}

2.1 Estructura y propiedades físico-químicas de las montmorillonitas

\subsubsection{Estructura cristalina}

Los minerales arcillosos son componentes naturales del suelo. Se clasifican como filosilicatos ya que presentan estructura laminar (phyllon $=$ hoja). Su tamaño es coloidal, distinguiéndose de otros materiales coloidales debido a las formas de partícula (anisométrica y frecuentemente irregulares); una distribución de tamaño de partícula muy ancha; los diferentes tipos de cargas eléctricas que presentan (cargas permanentes en las láminas y cargas variables dependientes del $\mathrm{pH}$ en los bordes); la capacidad de intercambio catiónico; la flexibilidad de las láminas y los distintos modos de agregación [Heller-Kallai et al., 2006].

Los filosilicatos en general, presentan una estructura basada en el apilamiento de planos de iones oxígeno e hidroxilos asociado a $\mathrm{Si}$ y $\mathrm{Al}$ conformando tetraedros y octaedros, respectivamente. Los grupos tetraédricos $\left(\mathrm{SiO}_{4}\right)^{4-}$ se unen compartiendo tres de sus cuatro oxígenos con otros vecinos formando capas, de extensión infinita y fórmula $\left(\mathrm{Si}_{2} \mathrm{O}_{5}\right)^{2-}$ que constituyen la unidad fundamental de los filosilicatos. En ellas, los tetraedros se distribuyen formando hexágonos. Estas capas tetraédricas (o siloxánicas) se unen a otras octaédricas de óxidos de aluminio $\left(\mathrm{Al}_{2} \mathrm{O}_{6}\right)^{6-}$. El plano de unión entre ambas capas está formado por los 
átomos de oxígeno de los tetraedros que no están compartidos con otros tetraedros (oxígenos apicales) y por grupos $\mathrm{OH}^{-}$de la capa octaédrica, de forma que, en este plano queda un grupo $\mathrm{OH}^{-}$en el centro de cada hexágono formado por 6 oxígenos apicales. Una unión similar puede ocurrir en la superficie opuesta de la capa octaédrica. Así, los filosilicatos pueden estar formados por dos capas -tetraédrica y octaédrica- y se denominan bilaminares, 1:1 o T:O; o bien por tres capas -una octaédrica y dos tetraédricasdenominándose trilaminares, 2:1 o T:O:T. A la unidad formada por la unión de una capa octaédrica más una o dos tetraédricas se la denomina lámina.

Las esmectitas son filosilicatos 2:1. Normalmente, $\mathrm{Si}^{4+}, \mathrm{Al}^{3+}$ y $\mathrm{Fe}^{3+}$ ocupan sus sitios tetraédricos. La sustitución de un $\mathrm{Si}^{4+}$ por un catión $\mathrm{R}^{3+}$ en el sitio tetraédrico crea un exceso de carga negativa en los oxígenos basales y en el oxígeno apical. Esto afecta la carga total (basal y de borde) de la lámina 2:1 así como también la carga negativa local en la superficie o base de la lámina. Los sitios octaédricos pueden ocuparse por cationes trivalentes $\left(\mathrm{R}^{3+}\right)$, normalmente $\mathrm{Al}^{3+} \mathrm{o} \mathrm{Fe}^{3+}$ en dos sitios octaédricos, con una vacancia en el tercer sitio $\left[\left(\mathrm{R}^{3+}\right)_{2}(\mathrm{OH})_{6}\right]$; o por cationes divalentes $\left(\mathrm{R}^{2+}\right)$ normalmente $\mathrm{Fe}^{2+}, \mathrm{Mg}^{2+} \mathrm{O} \mathrm{Mn}^{2+}$ en todos los sitios octaédricos $\left[\left(\mathrm{R}^{2+}\right)_{3}(\mathrm{OH})_{6}\right]$. Cuando los cationes mayoritarios en la capa octaédrica son los $\mathrm{R}^{2+}$, se denominan esmectitas trioctaédricas; mientras que cuando los cationes mayoritarios son los $\mathrm{R}^{3+}$, se denominan esmectitas dioctaédricas. La sustitución de $\mathrm{R}^{3+} \mathrm{O} \mathrm{R}^{2+}$ por cationes de menor estado de oxidación y la presencia de vacancias en la capa octaédrica genera un exceso de carga negativa en las láminas.

Las cargas negativas permanentes, originadas por las sustituciones isomórficas que ocurren naturalmente por procesos geológicos en las capas tetraédricas y octaédrica, se balancean en las esmectitas por la presencia en el espacio interlaminar (espacio existente entre dos láminas consecutivas) de cationes inorgánicos. La naturaleza química de estos cationes (radio iónico, entalpía de hidratación, etc.) determinan en parte las propiedades físico-químicas del mineral. Una característica común de estos cationes es su entalpía de hidratación, cuyos valores se encuentran en el rango de -300 a $-1500 \mathrm{~kJ} / \mathrm{mol}$. Como resultado, estos cationes se presentan siempre con una esfera de hidratación completa o parcial, lo que aporta a la hidrofilicidad de estos minerales [Heller-Kallai et al., 2006].

En particular, la montmorillonita es una esmectita dioctaédrica, y sus cationes interlaminares más frecuentes son alcalinos $\left(\mathrm{Na}^{+}\right.$y $\left.\mathrm{K}^{+}\right)$o alcalinotérreos $\left(\mathrm{Mg}^{2+}\right.$ y Ca $\left.{ }^{2+}\right)$. $\mathrm{La}$ Figura 2.1 muestra la estructura cristalina de la montmorillonita y su composición general. 


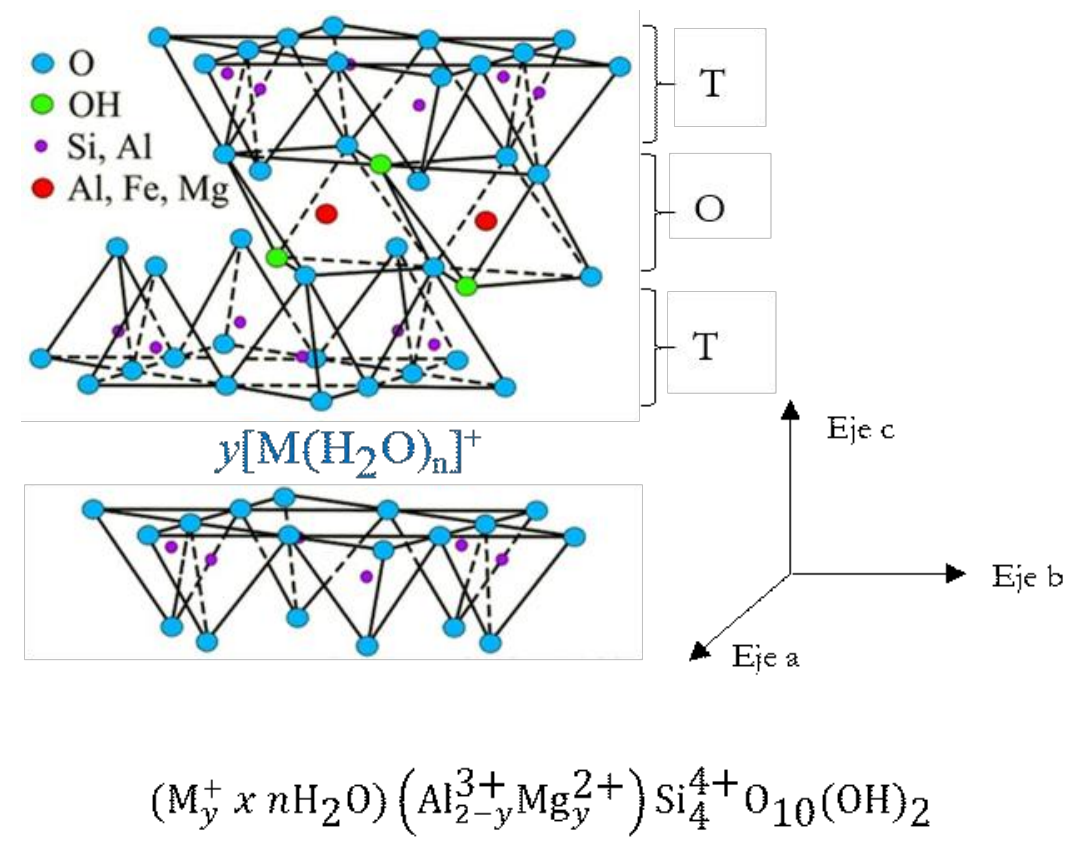

Figura 2.1 Estructura cristalina y composición química de la montmorillonita. T: capa tetraédrica, O: capa octaédrica.

La unidad formada por una lámina más la interlámina o intercapa es la unidad estructural de la montmorillonita. El espaciado basal es la distancia entre láminas a lo largo del eje c (Figura 2.1) y puede ser medido por difracción de rayos X (DRX).

La DRX es el resultado de la interacción de radiación electromagnética (en la región de los rayos X) con los átomos de un sólido, cuando las distancias entre éstos en la estructura cristalina es comparable con la longitud de onda de la radiación. En ciertas direcciones, (ángulos de incidencia, $\theta$ ) los rayos dispersados elásticamente interfieren constructivamente. Estos valores de $\theta$, la longitud de onda de los rayos y el espaciado de la red $\left(\mathrm{d}_{\mathrm{hk}}\right)$ están relacionados por la ecuación de Bragg:

$$
n \lambda=2 d_{h k l} \operatorname{sen} \theta
$$

(Ecuación 2.1)

El difractograma es un gráfico de intensidad de difracción en función del ángulo de Bragg, 2 $\theta$. Dependiendo de las condiciones de interferencia cada plano de átomos produce una serie de $n$ reflexiones, a partir de los cuales puede calcularse la distancia interlaminar empleando la ecuación de Bragg (Ecuación 2.1). Cada compuesto tiene su propio set característico de valores $\mathrm{d}_{\mathrm{hkl}}$ e intensidades, las cuales son utilizadas para su identificación [Lifshin, 1999; Ladd y Palmer,. 2003]. La interpretación cualitativa de un difractograma consiste en la identificación de las especies cristalinas, la cual se realiza comparando dicho difractograma con el de patrones. 
El pico $\mathrm{d}_{001}$ corresponde, en las montmorillonitas, al espaciado basal, es decir, a la distancia existente entre dos láminas T:O:T que es igual a la suma del espesor de una lámina T:O:T más el espacio interlaminar. Es así que esta técnica permite medir el grado de expansión o hinchamiento, lo cual en el caso de las esmectitas es función del catión interlaminar. En este tipo de minerales, la distancia mínima entre láminas es 0,96 nm [Emmerich et al., 2001] y se determina en la montmorillonita deshidratada. En condiciones de humedad y a temperatura ambiente, este valor varía en función del catión interlaminar y de su grado de hidratación. Además, la incorporación de cationes orgánicos en la intercapa, dependiendo de la concentración y de su volumen molecular, provoca el incremento del espaciado basal que se traduce en corrimientos del pico $\mathrm{d}_{001}$ hacia menores ángulos.

La DRX se utilizó a lo largo de este trabajo de investigación con el objetivo de comprobar la intercalación de distintos compuestos en la zona interlaminar de las montmorillonitas, analizando el cambio en la posición del pico de reflexión $\mathrm{d}_{001}$ en los sucesivos experimentos de adsorción.

\subsubsection{Cargas superficiales}

Las montmorillonitas presentan dos tipos de cargas superficiales: las cargas permanentes, debidas a las sustituciones isomórficas y vacancias en las láminas T:O:T y cargas variables dependientes del $\mathrm{pH}$ del medio.

Las cargas permanentes, aparecen como resultado de las sustituciones isomórficas en las capas tetraédricas y octaédricas, y por vacancias en la capa octaédrica -como se explicó en la Sección 2.1.1- y se localizan en las caras interlaminares y externa. Estas cargas estructurales son independientes del $\mathrm{pH}$ y generan un importante campo eléctrico negativo en las superficies basales.

Las cargas variables se originan por la interrupción de la red tridimensional y se sitúan en los bordes de las láminas. El desarrollo de estas cargas se deriva de la adsorción o la disociación de protones y por lo tanto, su signo y densidad dependen del $\mathrm{pH}$ de la dispersión. En un medio ácido, un exceso de protones crea cargas de borde positivas, y su densidad disminuye con el aumento de $\mathrm{pH}$. Las cargas negativas son producidas por la disociación de los grupos silanol (Si-OH) y aluminol (Al-OH) [Heller-Kallai et al., 2006]. Las reacciones ácido-base que ocurren en los bordes se esquematizan en la Figura 2.2

$$
\begin{aligned}
& \equiv \mathrm{SOH}_{2}^{+} \leftrightarrow \equiv \mathrm{SOH}+\mathrm{H}^{+} \\
& \equiv \mathrm{SOH} \leftrightarrow \equiv \mathrm{SO}^{-}+\mathrm{H}^{+}
\end{aligned}
$$

Figura 2.2 Equilibrios ácido-base en los sitios de bordes de la montmorillonita. 
donde $\equiv$ SOH representa los grupos hidroxilo de borde.

Este comportamiento anfótero de los bordes de la montmorillonita, junto a las cargas estructurales en los sitios basales, genera una heterogeneidad de cargas que afectan los modos de asociación de las láminas y su comportamiento coloidal. Es por ello que surgen algunos parámetros de especial interés para caracterizar y comprender el comportamiento de las montmorillonitas en suspensión. Por ejemplo, el punto de cero carga $\left(\mathrm{pH}_{\mathrm{PZC}}\right)$ es el $\mathrm{pH}$ al cual la carga eléctrica neta de la superficie está equilibrada (vale cero). Otro parámetro interesante para entender la reactividad de los bordes es el $\mathrm{pH}$ al cual la carga neta de los mismos es neutra. Este valor se denomina comúnmente punto de cero carga de borde $\left(\mathrm{pH}_{\mathrm{PZC} \text {, borde }}\right)$. A valores de $\mathrm{pH}$ por debajo de $\mathrm{pH}_{\mathrm{PZC} \text {, borde }}$ la carga neta de bordes es positiva porque los grupos cargados positivamente exceden a aquellos cargados negativamente, mientras que a $\mathrm{pH}$ superiores a $\mathrm{pH}_{\mathrm{PZC} \text {, borde }}$ ocurre lo contrario.

Experimentalmente no es posible medir la carga superficial de una partícula, por lo que se recurren a diferentes técnicas que permiten aproximarse a ese valor. Una manera de estimarla es midiendo el potencial zeta de una suspensión. Cuando la montmorillonita se suspende en agua, como cualquier partícula coloidal, desarrolla una red de cargas eléctricas en su superficie. Según el modelo de la doble capa eléctrica, se forma una capa de contraiones (iones de carga opuesta a la de la partícula, positiva) llamada capa de Stern donde el potencial eléctrico decae linealmente con la distancia; fuera de esta capa "fija", en un área nebulosa o capa difusa, hay distintas composiciones de co-iones (iones de carga igual a la de la partícula, negativa) y el decaimiento del potencial eléctrico con la distancia es exponencial. El espesor de esta doble capa depende del tipo y concentración de los iones de la solución [Tschapek, 1989]. El potencial en el plano donde se unen la capa difusa y la de Stern se conoce como potencial zeta y es de especial importancia ya que puede ser medido de manera muy simple, por microelectroforesis, potencial de difusión o potencial de streaming [Lützenkirchen et al., 2014; Li et al., 2016a; 2016b] y otorga información sobre la distribución de los iones en la interfase sólido/líquido, e indirectamente, sobre la carga superficial del mineral.

Las medidas de potencial zeta basadas en la técnica de microelectroforesis, consisten en someter a una suspensión a la acción de un campo eléctrico; como consecuencia, las partículas migran en el campo y su velocidad y sentido están relacionados con su potencial zeta a través de la ecuación de Helmholtz-Smoluchowski [Hunter et al., 1981]. Sin embargo, la relación entre la movilidad electroforética y el potencial zeta es bastante más complicada por diversos motivos, entre ellos la influencia de la conductividad en la 
superficie, el efecto de conductividad del líquido en los poros en el caso de materiales porosos, la geometría no esférica de los minerales de arcilla, etc. En ese sentido, el potencial zeta calculado a partir de la ecuación de Helmholtz-Smoluchowski no indica mucho más que la carga externa de las partículas y provee un valor proporcional a la movilidad electroforética [Heller-Kallai et al., 2006].

$\mathrm{El} \mathrm{pH}$ al cual el potencial zeta es cero se denomina punto isoeléctrico (IEP). En algunos casos dicho valor es igual a $\mathrm{pH}_{\mathrm{PZC}}$ pero cuando existen adsorciones específicas, los valores de IEP y $\mathrm{pH}_{\mathrm{PZC}}$ difieren y para determinarlos se deben utilizar distintas técnicas (determinación del potencial de difusión y movilidad electroforética, respectivamente o potencial de difusión y curvas de titulación potenciométrica) [Escudey et al., 1986; Torres Sánchez et al, 2001].

Las montmorillonitas poseen cargas estructurales que, al exceder en magnitud a las cargas de borde (debido a que el borde representa apenas el 1\% de la superficie total, mientras que las cargas dependientes del pH representan entre el 10 y el $20 \%$ de la carga total de superficie [Anderson y Sposito, 1991]), determinan el comportamiento electrocinético del mineral. Esto explica que el potencial zeta de las montmorillonitas sea negativo en todo el intervalo de $\mathrm{pH}$ y que no pueda determinarse su IEP mediante curvas de potencial zeta. Aun a aquellos valores de $\mathrm{pH}$ a los cuales se esperaría bordes cargados positivamente o en forma neutra ( $\mathrm{pH}$ entre 4 y 5,3 o menores ${ }^{3}$ [Pecini y Avena, 2013]), se observa potencial zeta negativo debido al efecto dominante de las cargas permanentes. El IEP de la montmorillonita medido por potencial de difusión tiene un valor de $\mathrm{pH}$ comprendido entre 3,0 y 4,8 [Lombardi et al., 2003].

Las medidas de potencial zeta se utilizaron a lo largo de este trabajo de investigación con el objetivo de comprobar los cambios a nivel de cargas superficiales tanto por la unión de alquilaminas, como de metales y de fungicidas.

\subsubsection{Capacidad de intercambio catiónico}

La presencia de cationes inorgánicos interlaminares y su posibilidad de intercambio es lo que amplió considerablemente el uso de las montmorillonitas en aplicaciones tecnológicas. La capacidad de intercambio catiónico (CIC) es la magnitud que cuantifica la tendencia de las montmorillonitas de sorber especies catiónicas de una solución, y se relaciona con la carga de capa [Thomas et al., 1999].

\footnotetext{
${ }^{3}$ Los autores determinaron que el $\mathrm{pH}$ PZC, borde de la montmorillonita está entre 4,0 \pm 0,2 y 5,3 \pm 0,2.
} 
Este valor se expresa como equivalentes de carga positiva por unidad de masa de arcilla, por ejemplo en $[\mathrm{mmol} / \mathrm{g}]$. En general, el intercambio entre cationes para compensar la carga negativa de las láminas cumple con las siguientes características:

(i) es reversible;

(ii) el paso limitante de la velocidad es la difusión;

(iii) es estequiométrico; y

(iv) en la mayoría de los casos prevalece la selectividad de un catión respecto de otro [Gast, 1977].

El punto (iv) es lo que permite medir la CIC de un material, ya que para determinarla experimentalmente, es necesario remplazar completamente todos los cationes inorgánicos de intercambio por cationes de referencia que no están presentes en la muestra de mineral. La selectividad por algunos cationes respecto a otros, se debe a diversos factores: esferas de hidratación de los cationes en la superficie y en la solución, interacciones electrostáticas catión-superficie y catión-catión, interacciones entre las moléculas de agua y la superficie, carácter de polarizabilidad de los cationes, factores entrópicos, etc. En general para las montmorillonitas, los cationes divalentes son preferidos respecto a los monovalentes, y los cationes orgánicos son preferidos respecto a los inorgánicos [Narine y Guy, 1981].

Actualmente existen una gran variedad de metodologías para medir la CIC. En todos los casos, se trata como se dijo más arriba, de desplazar los cationes inorgánicos interlaminares con un cation o complejo catiónico de referencia, en un volumen conocido de solución y $\mathrm{pH}$ y determinar analíticamente los cationes "salientes" mediante absorción atómica, espectrofotometría, titulaciones, etc. El valor obtenido depende de la naturaleza de los cationes de intercambio, del tamaño de partícula, de la temperatura, de las condiciones de fase (si se trabaja con una suspensión diluida o concentrada, en fase acuosa o empleando un solvente orgánico, o si se mide en reacciones en estado sólido), etc. Además, como algunos cationes también pueden complejarse con sitios de borde cuya carga depende del pH, es muy importante determinar el pH al cual se la mide [Heller-Kallai et al., 2006].

\subsubsection{Sitios activos en montmorillonitas en la interacción con materia orgánica}

Las montmorillonitas pueden participar de una diversidad muy amplia de reacciones con compuestos orgánicos: las moléculas de agua en su espacio interlaminar pueden ser desplazadas por moléculas orgánicas polares; los cationes interlaminares pueden formar complejos con ligandos orgánicos neutros o ser intercambiados por diversos tipos de cationes orgánicos (iones de alquilamonio, por ejemplo). En cada uno de estos procesos, la 
fuerza impulsora es el desarrollo de interacciones (uniones hidrógeno, interacciones iondipolo, enlaces de coordinación, de van der Waals [Weiss, 1963; Theng, 1974; Lagaly, 1984; Jasmund y Lagaly, 1993; Yariv y Cross, 2002]) entre las moléculas y sitios específicos de la superficie del mineral, denominados sitios activos.

El concepto de sitio activo ha demostrado ser una herramienta muy útil para describir la reactividad de macromoléculas como las enzimas. Luego, este concepto se trasladó para describir la estructura y función de la superficie de los minerales de arcilla y aquí se lo introduce para comprender la complejidad de la química superficial de las montmorillonitas en su interacción con la materia orgánica. El término sitio activo implica un proceso en donde una reacción química de superficie se origina a escala molecular propia de la superficie del mineral. Se definen por su composición química, por el arreglo geométrico de los átomos en la superficie y por su reactividad [Johnston, 1996].

Los cinco sitios activos en las montmorillonitas son los siguientes:

\section{(i) Superficie siloxánica}

En ausencia de sustituciones isomórficas y defectos en las láminas, la superficie siloxánica de un mineral arcilloso está compuesto por átomos de $\mathrm{O}$ involucrados en enlaces covalentes Si-O. En estas condiciones, dicha superficie es neutra e hidrofóbica, por lo tanto no es capaz de formar uniones hidrógeno con moléculas de agua pero sí puede experimentar interacciones van der Waals con moléculas orgánicas poco o no polares. Es así que, en las organo-montmorillonitas donde los cationes inorgánicos interlaminares han sido intercambiados por otros, orgánicos, se tiene un doble aporte al carácter hidrofóbico: uno dado por la presencia de los cationes orgánicos entre las láminas y, el otro, dado por la superficie siloxánica.

(ii) Sitios de carga permanente

Estos sitios de carga negativa permanente se originan en las sustituciones isomórficas que ocurren naturalmente tanto en la capa tetraédrica como en la capa octaédrica de las montmorillonitas. Las sustituciones isomórficas polarizan a los átomos de oxígeno de la superficie promoviendo su interacción vía uniones hidrógeno con moléculas de agua [Heller-Kallai et al., 2006], y electrostáticamente con cationes o moléculas orgánicas polares.

Las cargas negativas en la lámina se distribuyen de diferente manera según la sustitución sea en la capa octaédrica o en la tetraédrica. En el caso de la sustitución de $\mathrm{Al}^{3+}$ por $\mathrm{Mg}^{2+}$ en las capas octaédricas, la distribución de cargas se encuentra deslocalizada sobre una región cercana al plano de los átomos de oxígeno. Si el reemplazo es en la capa tetraédrica 
$\left(\mathrm{Si}^{4+}\right.$ por $\left.\mathrm{Al}^{3+}\right)$, la distribución de cargas resulta más localizada sobre los tres átomos de oxígeno.

El hecho de que los cationes orgánicos como las aminas cuaternarias presenten mayores energías de interacción electrostática que los cationes inorgánicos (debido a la presencia de las esferas de hidratación en los últimos, [Narine y Guy, 1981]) posibilita la intercalación de moléculas orgánicas en la estructura de las montmorillonitas mediante reacciones de intercambio catiónico. De esta manera se pueden obtener organo-montmorillonitas cuyas propiedades difieren a las del material de partida y serán explicadas en la Sección 2.2.

(iii) Sitios de carga variable

Estos sitios se originan en los defectos (bordes rotos, pliegues o agujeros) que ocurren naturalmente en las láminas T:O:T (idealmente infinitas), dejando expuestos los grupos Si$\mathrm{OH}$ y Al-OH. Estos sitios también aportan hidrofilicidad a las montmorillonitas [HellerKallai et al., 2006]. A valores de $\mathrm{pH}$ menores al $\mathrm{pH}_{\mathrm{PZC}}$, borde prevalecen en los bordes los grupos cargados positivamente, lo que se favorece su interacción con ácidos orgánicos u oxianiones. $\mathrm{A} \mathrm{pH}$ mayores al $\mathrm{pH}_{\mathrm{PZC} \text {, borde }}$ prevalecen las cargas neutras y negativas con lo cual la interacción se verá favorecida con especies neutras y catiónicas. La contribución total de estos sitios a la reactividad general de las montmorillonitas no es importante ya que, como se dijo anteriormente, representan sólo el 1\% de la superficie total. Sin embargo, Lagaly y Dekany [2005] encontraron que en reacciones de intercambio catiónico realizadas a $\mathrm{pH}$ neutros o ligeramente ácidos, la adsorción de cationes de alquilamonio en una cantidad mayor a la de la CIC ocurría con liberación de protones y se lo adjudicaron a la participación de los sitios de bordes en la unión con las aminas.

(iv) Cationes metálicos interlaminares y sus esferas de hidratación

Los cationes metálicos presentes en los sitios de carga permanente de la montmorillonita pueden actuar como sitios activos, uniéndose a compuestos orgánicos directamente o a través de uniones de hidrógeno de sus esferas de hidratación. El tipo de unión está determinado principalmente por la dureza o blandura de los cationes según el principio de Ácidos y Bases, Duros y Blandos (ABDB) ${ }^{4}$. Por ejemplo, se observó que

\footnotetext{
${ }^{4}$ Este principio fue presentado por Pearson [1963] para explicar la estabilidad de los complejos ácidobase. Plantea que los ácidos de Lewis pueden ser clasificados como blandos o duros según su polarizabilidad (lo cual depende de su carga y de su radio iónico). En su trabajo, muestra experimentalmente que los ácidos duros se unen preferentemente a bases duras o no-polarizables, mientras que los ácidos blandos se unen fuertemente a bases blandas. Las explicaciones a tales comportamientos se basan en el grado iónico o covalente del enlace ácido-base; la formación de enlaces $\pi$; el fenómeno de correlación electrónica y efectos
} 
aminas alifáticas y aromáticas se coordinan directamente con cationes blandos como $\mathrm{Zn}^{2+}$, $\mathrm{Cd}^{2+}, \mathrm{Cu}^{2+} \mathrm{y} \mathrm{Ag}^{+}$, mientras que forman puentes de agua con cationes duros (iones alcalinos, alcalinos-térreos y $\mathrm{Al}^{3+}$ ) en la intercapa, aunque estos últimos no son complejos estables en solución [Farmer y Mortland, 1966]. También se observó que el vapor de benceno reacciona con $\mathrm{Cu}^{2+}$ en la intercapa de la montmorillonita desplazando una parte del agua de hidratación, a través de la interacción de los electrones $\pi$ del benceno con el catión [Doner y Mortland, 1969; Pinnavaia et al., 1971; 1974; Rupert, 1973; Lagaly, 1984].

Además de la formación de enlaces de coordinación es posible el desarrollo de interacciones electrostáticas entre el catión interlaminar y una molécula orgánica polar. Por ejemplo, la adsorción de metanol en montmorillonitas con $\mathrm{Li}^{+}$y $\mathrm{Ca}^{2+}$ en su intercapa, se desarrolla mediante interacciones ion-dipolo entre las moléculas de alcohol y los cationes interlaminares [Annabi-Bergaya et al., 1981].

Es importante señalar que las moléculas de agua coordinadas a los cationes interlaminares presentan propiedades físico-químicas diferentes a las de las moléculas de agua en solución, y pueden ser considerablemente más ácidas que estas últimas debido a su proximidad al catión. Esto genera que, por ejemplo, la relación de concentraciones de las especies protonada y sin protonar de una base difiera en el espacio interlaminar con dicha relación en solución [Karickhoff y Bailey, 1976]. La protonación en la intercapa también se ve reforzada por la capacidad de la superficie negativa del mineral de arcilla de estabilizar la forma protonada de la base, disminuyendo su potencial químico respecto al de su forma neutra [Feldkamp y White, 1979].

\section{(v) Superficie externa}

Aunque la superficie externa de la montmorillonita no se diferencie de la superficie interlaminar (sitios de carga permanente y superficie siloxánica) en composición química ni distribución geométrica de los átomos, se la introduce aquí como un sitio activo diferente a los de la superficie interlaminar por su reactividad química. Son diversos los ejemplos en la literatura científica que muestran cómo los sitios de superficie externa reaccionan de manera diferente a los sitios de superficie interna, tanto cuando se trata de reacciones de adsorción en fase acuosa, como de adsorción o desorción de gases.

Diversos autores concluyen, en base a isotermas de adsorción, cálculos matemáticos, DRX, etc., que la unión de determinados compuestos orgánicos ocurre en la superficie

de solvatación. Es de naturaleza fenomenológica ya que describe una gran gama de fenómenos químicos pero de un modo cualitativo y no cuantitativo. No es infalible; existen muchas discrepancias aparentes y excepciones [Pearson, 1998]. 
externa e interna en dos etapas diferenciadas. Ya por el 1979, Annabi-Bergaya et al. señalaban que alrededor de la mitad del total de moléculas adsorbidas (metanol e isopropanol) sobre montmorillonitas cálcicas estaban unidas a los microporos formados entre partículas del mineral y no en el espacio interlaminar [Annabi-Bergaya et al., 1979]. Otro ejemplo, es la adsorción del pesticida picloram, de naturaleza aniónica, que se une en primer instancia a los sitios de superficie externa y luego, en una segunda instancia menos favorecida desde el punto de vista energético, ingresa en los sitios interlaminares [MarcoBrown et al., 2014]. En el mismo sentido, muchos autores concluyeron que la adsorción del colorante catiónico azul de metileno ocurre en un primer paso en los sitios de superficie externa y cuando la concentración en este área aumenta lo suficiente se forman aglomerados lo cual induce la migración del compuesto a los sitios interlaminares [Breen, y Loughlin, 1994; Breen y Rock, 1994; Neumann et al., 2002].

La diferencia en la reactividad también se evidencia en los procesos de hidratación y deshidratación de muestras en polvo. El agua unida a la superficie externa de la montmorillonita se desorbe más fácilmente (a menor temperatura) que el agua presente en el espacio interlaminar [Mackenzie, 1970].

\subsection{Obtención, estructura y propiedades físico-químicas de organo- montmorillonitas}

La modificación de la superficie de los minerales de arcilla ha recibido gran atención debido a que permite la creación de nuevos materiales, ampliando sus aplicaciones. Hay diversas vías de modificación de minerales de arcilla como adsorción, intercambio catiónico con cationes orgánicos e inorgánicos, unión en los bordes de aniones orgánicos e inorgánicos, grafting de compuestos orgánicos, reacción con ácidos, pilareado con diferentes tipos de poli-(hidroxo) cationes metálicos, polimerización intra-partícula, deshidroxilación y calcinación, delaminación y re-agregación de montmorillonitas, liofilización, etc. [Bergaya y Lagaly, 2001].

En particular, la modificación de minerales de arcilla por intercambio catiónico con sales de aminas cuaternarias ha generado una importante expansión de sus aplicaciones iniciales [de Paiva et al., 2008], por ejemplo, para su uso como adsorbente selectivo y agente de control reológico como espesantes o gelificantes. Actualmente, una importante aplicación de las organo-arcillas es en nano-compositos poliméricos [Markarian, 2005]. Pero además de los usos generalizados, la facilidad de este tipo de modificación con miras hacia aplicaciones específicas generó un aumento de las investigaciones sobre estos materiales, que queda evidenciado en el número creciente de publicaciones científicas en los últimos años. 
Diversos tipos de compuestos orgánicos han sido investigados en la obtención de organo-arcillas: surfactantes no iónicos como los alcoholes lineales etoxilados, éteres corona, sistemas aromáticos como sales de imidazoles y quinolinas, sales alquilfosfónicas, sales de anilinas, etc., pero las sales de alquilamonio cuaternario son, sin duda, las más utilizadas. Estas últimas, son surfactantes catiónicos que se sintetizan mediante la alquilación de aminas. Son preferidas por sobre las sales de alquilamonio primario en los usos industriales debido a que no experimentan hidrólisis (equilibrio alquilamonio/alquilamina) y consecuentemente la desorción de alquilamina libre se reduce [Favre y Lagaly, 1991].

Entre todos los minerales de arcilla, las esmectitas y especialmente las montmorillonitas han sido muy investigadas en la preparación de organo-arcillas debido a sus excelentes propiedades como gran área superficial, capacidad de intercambio catiónico y de hinchamiento. Dependiendo de la carga de capa de la montmorillonita (densidad de cationes interlaminares y consecuente empaquetamiento de los cationes alquilamonio), del largo de las cadenas alquílicas del surfactante y de su porcentaje intercambiado, las cadenas hidrocarbonadas adoptan distintos arreglos entre las láminas. Se trata de conformaciones en las cuales las cadenas se ubican de forma paralela a éstas ya sea formando mono- o bicapas; o inclinadas respecto a éstas, formando estructuras tipo pseudo-tricapa o parafínicas (Figura 2.3). En particular, la conformación pseudo-tricapa es energéticamente inestable debido a la repulsión electrostática entre las cabezas cargadas y es por ello que las cadenas adoptan arreglos tipos parafina. Además, el arreglo tipo parafina permite que los grupos amonio se ajusten mejor a los sitios de carga permanente [de Paiva et al., 2008]. En este arreglo, el ángulo formado por las cadenas y las láminas siloxánicas de la montmorillonita aumenta cuando aumenta la carga de la capa. Por lo tanto, el aumento del espaciado basal al incrementar el largo de cadena varía con la densidad de carga de la lámina.

La adsorción de moléculas orgánicas en la superficie de la montmorillonita genera la aparición de nuevos sitios activos hidrofóbicos en la arcilla, que las hace más proclives a una adsorción posterior de moléculas orgánicas poco polares [Heller-Kallai et al. 2006; Xi, 2006]. Particularmente, cuando la montmorillonita es modificada por intercalación y fijación en la superficie externa de cationes orgánicos de cadena larga, se forma una fase orgánica que hace a las veces de medio de partición para compuestos orgánicos no-iónicos. Mecanísticamente, el proceso de adsorción en estos casos se propone que ocurra de manera similar a la partición de estos compuestos en el bulk de un solvente orgánico no polar como el hexano o el octanol, excepto porque en las organo-montmorillonitas la fase orgánica de partición está fijada en la superficie del mineral [Kowalska, 1994]. 


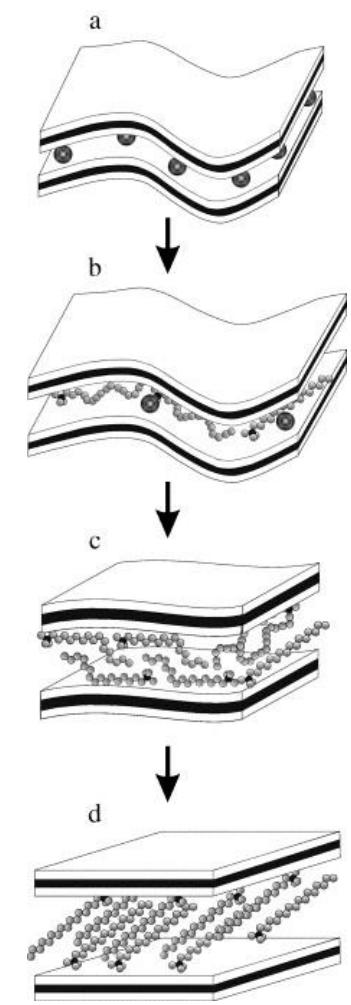

Figura 2.3 Esquema de las láminas curvadas de la montmorillonitas transformándose en láminas planas con el agregado de surfactante y empaquetamiento de las cadenas alquílicas en la intercapa. (a) Montmorillonita sódica, (b) arreglo monocapa, (c) pseudo-tricapa, (d) tipo parafina. Fuente: [He et al., 2006].

Los cambios estructurales producidos por la intercalación de compuestos orgánicos en la montmorillonita, pueden seguirse mediante una multiplicidad de técnicas experimentales, muchas de las cuales se utilizan a lo largo de esta tesis. En particular, la incorporación de compuestos orgánicos y su arreglo en la intercapa pueden inferirse mediante DRX a partir de la posición del pico $\mathrm{d}_{001}$ en los difractogramas; la unión de cationes orgánicos en la superficie externa y bordes de la arcilla puede conocerse mediante curvas de potencial zeta en función del $\mathrm{pH}$; la interacción de la materia orgánica con los sitios activos de la montmorillonita, así como el contenido orgánico en la muestra, pueden estimarse a partir de técnicas de análisis térmicos.

El análisis térmico engloba un conjunto de métodos analíticos que estudian los cambios de una propiedad cuando una muestra es sometida a un programa de temperatura en una atmósfera controlada [Skoog, 2001]. La introducción de compuestos orgánicos en las arcillas conduce a cambios estructurales y de composición. Al ser sometidas a calentamiento o enfriamiento, los procesos de fusión, solidificación, cristalización, oxidación, descomposición, transición de fases, expansión, sinterización, etc., se modifican respecto a la muestra natural, y se evidencian en modificaciones en las curvas de análisis 
térmicos. Es por ello que éstas técnicas se utilizan para identificar organo-arcillas y diferenciarlas de las arcillas puras. Por este motivo, las curvas de análisis térmicos son consideradas por algunos autores como "huellas dactilares" de las arcillas [Dultz, 2005].

Entre las múltiples técnicas de análisis térmico que existen, las que se utilizaron en esta tesis son el análisis térmico diferencial (ATD) y el análisis termogravimétrico (Tg) que se determinaron experimentalmente en forma simultánea.

El ATD consiste en medir la diferencia de temperaturas que se establece entre la muestra y un material de referencia (inerte desde el punto de vista físico, químico y térmico), en función del tiempo o de la temperatura, cuando ambas, muestra y referencia, se someten al mismo programa de temperaturas en una atmósfera controlada. Esta diferencia de temperaturas es originada por reacciones que ocurren en la muestra con liberación o absorción de calor (procesos exotérmicos o endotérmicos, respectivamente). El ATD fue la primera técnica de análisis térmico, que se utilizó para caracterizar y distinguir cualitativamente arcillas naturales ya que cualquier transformación física y/o química se produce con un efecto térmico, lo que provoca una diferencia entre la temperatura de la muestra y la de la referencia. Por lo tanto, los respectivos picos endo- o exotérmicos que se observan en las curvas ATD son una forma muy práctica para identificar estas transformaciones y se han utilizado como una de las principales herramientas para la caracterización cualitativa de arcillas [Dweck, 2008].

$\mathrm{La} \mathrm{Tg}$ es la técnica que permite estudiar las pérdidas de masa de una muestra sometida a un programa de temperaturas en una atmósfera controlada. Los instrumentos modernos determinan en forma simultánea ATD y Tg. Este hecho permite asociar los procesos exoo endotérmicos de las curvas ATD con pérdidas de masa en $\mathrm{Tg}$ y diferenciar estos procesos de aquellos que implican transiciones de fase (sin pérdida de masa asociada). Si además se grafica la derivada primera de la curva $\mathrm{Tg}$ (DTg), se puede obtener la temperatura a la cual comienza la reacción y la temperatura a la cual la variación de masa respecto de la temperatura es máxima (máximo del pico DTg).

Las montmorillonitas presentan en ATD dos picos endotérmicos asociados a pérdidas de masa en $\mathrm{Tg}$ por procesos de deshidratación y picos tipo $\mathrm{S}$ a mayores temperaturas debido a cambios de fase del mineral.

El primer salto en la curva Tg, entre $25-200{ }^{\circ} \mathrm{C}$ aproximadamente (Región I), resulta de la pérdida de moléculas de agua fisisorbidas y unidas a cationes interlaminares. Esto conduce generalmente a dos picos DTg: el primero, a menores temperaturas, corresponde a la desorción de moléculas de agua débilmente unidas a la superficie externa, o poros de 
las arcillas; el segundo, corresponde a moléculas de agua coordinadas a los cationes interlaminares, por lo que la temperatura a la cual este proceso ocurre es mayor y está condicionada por la naturaleza química de los cationes. El porcentaje de masa perdido en toda la etapa depende del contenido de cationes interlaminares hidratados (porcentaje respecto de la CIC) y del grado de hidratación de la arcilla [Mackenzie, 1970].

La segunda etapa de pérdida de masa en la montmorillonita, teóricamente entre 500-750 ${ }^{\circ} \mathrm{C}$ (Región III), corresponde a la pérdida de agua estructural, un proceso denominado deshidroxilación, esto es, la pérdida de grupos $\mathrm{OH}$ de la estructura T:O:T de la arcilla. Algunos autores sostienen que la deshidroxilación de la montmorillonita comienza a los $380{ }^{\circ} \mathrm{C}$. A esta temperatura el proceso es muy lento pero a medida que la temperatura aumenta, la velocidad también crece [Keidar et al., 2015]. El rango de temperaturas de deshidroxilación de una arcilla y su respectiva cinética depende de las características de los cationes interlaminares y de cómo los grupos hidroxilo interactúan con éstos.

Por último, en general entre $750-1000{ }^{\circ} \mathrm{C}$ (Región IV), se observan en ATD curvas S (picos exo-endo térmicos), con pérdidas de masa menores al 0,1\%, asociadas a cambios de fase del mineral (a espinela, cristobalita y mulita) [Hedley et al., 2007].

La intercalación de compuestos orgánicos en las montmorillonitas provoca diversos cambios en las curvas ATD, Tg y DTg.

En la Región I, algunos picos disminuyen su intensidad, desaparecen o cambian su temperatura. Esto se debe a la hidrofobicidad de la muestra (que conduce a una disminución del contenido de agua fisisorbida) y al intercambio de cationes inorgánicos hidratados por cationes orgánicos que no se encuentran coordinados a moléculas de agua.

En la Región II, esquemáticamente entre 200-500 ${ }^{\circ} \mathrm{C}$ aparecen nuevos picos exotérmicos y endotérmicos asociados a la combustión, descomposición, deshidratación, vaporización, fusión y/o sublimación de la materia orgánica. Debido a la interacción con el mineral arcilloso, las propiedades térmicas de los cationes o moléculas orgánicas difieren respecto de las sales orgánicas puras [Dultz et al., 2005]. Es así que la interpretación de los picos ATD y las pérdidas de masa asociadas a la descomposición del contenido orgánico no es simple. Algunos autores relacionan los saltos observados en Tg y las temperaturas de los picos DTg, con la combustión de moléculas orgánicas unidas a las capas siloxánicas de la montmorillonita a través de distintas fuerzas de interacción (electrostática, van der Waals, etc.) [Xi, 2004; Vazquez et al., 2008; Ouellet-Plamodon et al., 2014]. Otros autores, que se basan en resultados de técnicas en las cuales se anexa a los equipos ATD/Tg un analizador de los gases desprendidos (un espectrómetro de masas o un espectrómetro de infrarrojo) 
sostienen que los picos D'Tg y ATD que se observan en organo-montmorillonitas se deben, cuando se trabaja en atmósfera oxidante, a la oxidación de los elementos $\mathrm{H}$ a $\mathrm{H}_{2} \mathrm{O}, \mathrm{C}$ a $\mathrm{CO}_{2}$ y $\mathrm{N}$ a $\mathrm{NO}_{2}$. Estos procesos ocurren a distintas temperaturas y, según la disponibilidad de oxígeno, pueden ir acompañados con la formación de carbón. Este carbón puede sufrir transformaciones de fase con la temperatura y finalmente oxidarse, generando nuevos picos en el ATD, DTg y saltos en la curva Tg [Yariv, 2004; Keidar et al., 2015].

Por último, en la Región III, la interacción de los compuestos orgánicos con los $\mathrm{OH}$ estructurales de las arcillas conduce a cambios en la temperatura del proceso de deshidroxilación y la aparición de nuevos picos exotérmicos vinculados a la última etapa de oxidación de la materia orgánica.

Es importante resaltar que la temperatura a la cual comienza y termina cada uno de los procesos depende de la muestra y de las condiciones del ensayo, por eso las regiones que arriba se definieron son esquemas para interpretar los resultados. En muestras reales cada una de estas regiones a su vez puede presentar diferentes pasos o etapas de pérdida de masa, asociados a diferentes reacciones térmicas. 


\section{Capitulo 3. \\ Adsorción de imazalil \\ en montmorillonita y organo-montmorillonitas.}

\subsection{Introducción y objetivos específicos}

Diferentes organismos en el mundo establecen los límites máximos de residuos (LMR) de plaguicidas en alimentos. En el caso particular del imazalil (IMZ) el SENASA, en su Res. $\mathrm{N}^{\mathrm{o}} 256 / 03$, define que el LMR en cítricos es $5 \mathrm{mg} / \mathrm{Kg}$ y en frutas de pepita $2 \mathrm{mg} / \mathrm{Kg}$ [SENASA, 2003], en concordancia con los límites establecidos por organismos internacionales tales como la Unión Europea [PPDB, 2011], la Organización Mundial de las Naciones Unidas para la Alimentación y la Agricultura (FAO) y la Organización Mundial de la Salud [Codex Alimentarius].

Debido a estas restricciones, muchas investigaciones en la literatura científica nacional e internacional se orientan a desarrollar métodos efectivos de cuantificación y/o eliminación de IMZ de distintos frutos. Por ejemplo, Bovi Mitre et al. [2004] estudiaron la curva de degradación del fungicida en cáscaras de pomelos durante su almacenamiento en frío,

${ }^{5}$ Se agradece especialmente a Jana Madejová y a su grupo de investigación del Instituto de Química Inorgánica de la Academia de Ciencias de Eslovaquia (Bratislava, Eslovaquia) por abrirme las puertas de su laboratorio y de su conocimiento; y por su colaboración en la determinación de la CIC de la montmorillonita, contenido de carbono total y caracterización de muestras a través de las técnicas de FTIR. 
llegando a la conclusión que tras 34 días, la concentración del mismo en cáscara no supera el LMR.

Algunas de las metodologías de extracción reportadas implican un paso de adsorción en una matriz sólida. Por ejemplo, Barreto et al. [2011] caracterizaron y evaluaron el desempeño de un material de sílice mesoporoso MCM-41 como adsorbente en la extracción del pesticida de mango. Sus resultados fueron satisfactorios ya que lograron remover entre el 70 y 100\% de IMZ presente en los frutos, siendo estos valores comparables a aquellos obtenidos empleando sílica gel comercial como adsorbente [Barreto et al., 2011]. Otro ejemplo es el estudio de Ucles et al. [2015] donde se propone el uso de zirconia estabilizada con itria para la etapa de extracción en fase sólida del fungicida de naranjas y peras, obteniendo recuperaciones mayores o iguales al 90\%.

Además de los LMR en alimentos, la Council Directive de la Unión Europea [1998] en relación a la calidad de las aguas destinadas al consumo humano establece que la concentración total de pesticidas no puede superar $0,05 \mathrm{mg} / \mathrm{L}$. Debido a su baja biodegradabilidad, las plantas convencionales de tratamiento de aguas residuales no pueden eliminar sustancias como el IMZ [Santiago et al., 2015]. En ese sentido, una parte importante de la comunidad científica aboca sus estudios al desarrollo de metodologías para la remoción de IMZ de aguas. Entre las técnicas evaluadas se destacan, desde el punto de vista cuantitativo, aquellas que contemplan la degradación por fotocatálisis. Por ejemplo, Hacime et al., [2014] estudiaron la degradación de IMZ empleando métodos avanzados de oxidación ( $\mathrm{UV} / \mathrm{TiO}_{2}, \mathrm{UV} / \mathrm{K}_{2} \mathrm{~S}_{2} \mathrm{O}_{2}$ y UV $/ \mathrm{TiO}_{2} / \mathrm{K}_{2} \mathrm{~S}_{2} \mathrm{O}_{8}$ ) obteniendo resultados favorables a determinadas condiciones de $\mathrm{pH}$ en cada sistema. Por su parte, Santiago et al. [2015] evaluaron la remoción de IMZ de agua destilada y de efluentes sintéticos mediante fotocatálisis en presencia de $\mathrm{TiO}_{2}$ inmovilizado en diferentes soportes. Sus conclusiones fueron que cuando el $\mathrm{TiO}_{2}$ era soportado en borosilicatos y bajo la acción de luz solar se removía eficientemente IMZ a una concentración de $50 \mathrm{mg} / \mathrm{L}$ en efluentes sintéticos. En el trabajo de Jiménez-Tototzintle et al. [2015] contemplaron la remediación de efluentes de la industria agro-alimentaria por técnicas combinadas de biotratamientos seguido de fotocatálisis solar con $\mathrm{TiO}_{2} / \mathrm{H}_{2} \mathrm{O}_{2}$. Como resultados, encontraron que tanto el IMZ como el tiabendazol se removían en un $90 \%$ en presencia de peróxido de hidrógeno. Otros ensayos de degradación fotocatalítica de IMZ en aguas fueron reportados en los últimos años [Hazime et al., 2010; Dunia et al., 2011; Genena et al., 2011; Santiago et al., 2013; Carra et al., 2014]. 
La relación de cantidad de trabajos publicados sobre la eliminación de IMZ de matrices acuosas mediante fotocatálisis y aquellos que contemplan el uso de técnicas de adsorción, es asimétrica, siendo los últimos mucho menos abundantes que los primeros. Entre los trabajos que reportan técnicas de adsorción para la eliminación de IMZ de agua, vale destacar el artículo de Hu et al. [1998], donde utilizaron carbón activado para la adsorción de pesticidas aromáticos, alcanzando cantidades adsorbidas de IMZ de $1000 \mu \mathrm{mol} / \mathrm{g}(0,297$ $\mathrm{mg} / \mathrm{g}$ ). Por su parte, Martin-González et al. [2014] generaron tres carbones activados y compararon su desempeño como adsorbente de IMZ con un biosorbente y carbones activados comerciales. Los estudios cinéticos correspondientes indicaron que el paso determinante de la velocidad era la quimisorción del adsorbato en dichos materiales. La capacidad de adsorción máxima en el equilibrio que encontraron fue $411 \mathrm{mg} / \mathrm{g}$ para el carbón activado comercial en las condiciones: concentración inicial de IMZ $=100 \mathrm{mg} / \mathrm{L}$, $\mathrm{pH}$ inicial $=7 \mathrm{y}$ temperatura $=25^{\circ} \mathrm{C}$. Además, concluyeron que la adsorción de IMZ en estos materiales constituye un proceso favorable y espontáneo desde el punto de vista termodinámico.

No se hallaron a la fecha estudios de adsorción de IMZ en montmorillonitas ni organomontmorillonitas. En ese sentido, el trabajo de este capítulo buscará saldar ese vacío y comparar la eficiencia de remoción del fungicida por parte de una montmorillonita natural proveniente de la provincia de Río Negro y de organo-montmorillonitas obtenidas a partir de ésta y el surfactante catiónico bromuro de octadeciltrimetilamonio con los resultados de adsorción publicados en la literatura científica. Los objetivos específicos de este capítulo son:

(i) obtener organo-montmorillonitas mediante el intercambio catiónico con una amina cuaternaria (bromuro de octadeciltrimetilamonio) en diferentes porcentajes de intercambio respecto de la CIC;

(ii) caracterizar los materiales;

(iii) evaluar su capacidad como adsorbentes de IMZ;

(iv) caracterizar productos de adsorción de imazalil, dilucidar sitios activos involucrados y proponer un mecanismo de adsorción del fungicida en los adsorbentes estudiados;

(v) comparar la capacidad de remoción de los materiales sintetizados en el punto (i) respecto a la de la montmorillonita natural. 


\subsection{Materiales y métodos}

\subsubsection{Materiales}

El adsorbato utilizado fue un producto comercializado como XEDREL 50 ( Magan Argentina S.A., una suspensión acuosa muy insoluble, cuyo ingrediente activo es imazalil presente en un $47,2 \% \mathrm{p} / \mathrm{p}$. Debido a la densidad del formulado, las soluciones de IMZ se prepararon pesándolo y luego disolviéndolo en etanol grado analítico para alcanzar una concentración de $5000 \mathrm{mg} / \mathrm{L}$. Las soluciones más diluidas se prepararon a partir de esta solución madre empleando agua destilada.

Como adsorbentes se utilizaron una montmorillonita patagónica proveniente de la provincia de Río Negro, proporcionada por Castiglioni Pes y Cía (Mt) y organomontmorillonitas obtenidas a partir de ésta (OMts). La Mt fue caracterizada en trabajos previos [Lombardi et al., 2003; Magnoli et al., 2008] donde se determinó su punto isoeléctrico $=2,7$; superficie específica $=34 \mathrm{~m}^{2} / \mathrm{g}$; superficie específica total $=621 \mathrm{~m}^{2} / \mathrm{g}$; fórmula estructural $=\left[\left(\mathrm{Si}_{3,89} \mathrm{Al}_{0,11}\right)\left(\mathrm{Al}_{1,43} \mathrm{Fe}_{0,28}^{3+} \mathrm{Mg}_{0,30}\right) \mathrm{O}_{10}(\mathrm{OH})_{2}\right]-\mathrm{Na}_{0,41}{ }_{041}$. Su evaluación mineralógica, mediante la técnica cuantitativa de Rieltveld, indicó montmorillonita sódica como fase predominante (84\%) con cuarzo y feldespatos como fases minoritarias [Magnoli et al., 2008]. Su capacidad de intercambio catiónico, (CIC) $=0,8250 \pm 0,0007 \mathrm{mmol} / \mathrm{g}$, fue determinada mediante el método del Cu-trietilentetramina [Czímerová et al., 2006].

La obtención de las OMts, se realizó empleando bromuro de octadeciltrimetilamonio (ODTMA) grado analítico; fórmula química $=\left[\mathrm{C}_{18} \mathrm{H}_{37} \mathrm{~N}\left(\mathrm{CH}_{3}\right)_{3} \mathrm{Br}\right](\geq 97 \%)$; masa molecular $=392,5 \mathrm{~g} / \mathrm{mol}$; adquirido en Fluka (Buchs, Suiza). La estructura molecular de ODTMA se muestra en la Figura 3.1.

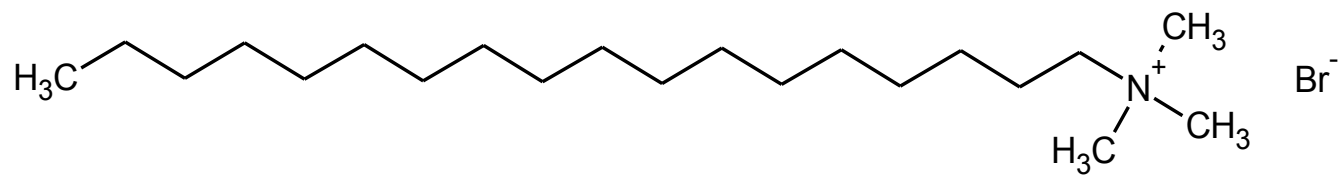

Figura 3.1 Estructura química de ODTMA.

El procedimiento de obtención de las OMts fue el siguiente: una cantidad de ODTMA equivalente al 39, 55, 105, o 211 \%CIC de la Mt se disolvió en $1 \mathrm{~L}$ de agua destilada a 60 ${ }^{\circ} \mathrm{C}$, se añadió lentamente $10 \mathrm{~g}$ de Mt y la suspensión resultante se mantuvo a $60{ }^{\circ} \mathrm{C}$ y en agitación durante $2 \mathrm{~h}$. El producto se centrifugó y lavó con agua destilada la cantidad de veces necesarias para remover el exceso de $\mathrm{Br}^{-}$(determinado mediante la prueba del $\mathrm{AgNO}_{3}$, test de Mohr). El sólido lavado se secó en estufa a $80{ }^{\circ} \mathrm{C}$ durante $72 \mathrm{~h}$. Antes de 
utilizarse, las muestras fueron molidas en mortero de ágata. Éstas se denominaron OMtX, donde X expresa el contenido final de ODTMA como porcentaje del valor de la CIC de la Mt.

\subsubsection{Métodos de caracterización}

En este capítulo se muestran las caracterizaciones de los adsorbentes y productos de adsorción de IMZ (llamados IMZ-Mt o IMZ-OMtX) mediante difracción de rayos X (DRX), potencial zeta, análisis térmico diferencial/termogravimetría (ATD/Tg), espectroscopía infrarroja (FTIR), cuantificación de carbono orgánico total y diámetro aparente (DAp).

\subsubsection{Difracción de rayos $X$}

Se analizaron muestras semiorientadas (en polvo) utilizando un difractómetro Philips PW 1710 operando a $40 \mathrm{kV}$ y $30 \mathrm{~mA}$ con radiación $\mathrm{Cu} \mathrm{K}_{\alpha}$ en el intervalo $2<2 \theta\left(^{\circ}\right)<15$ con un conteo de $10 \mathrm{~s} /$ paso y tamaño de paso de $2 \theta=0,02^{\circ}$.

\subsubsection{Potencial zeta}

Las medidas se llevaron a cabo utilizando un equipo Brookhaven 90Plus/Bi-MAS con la función movilidad electroforética, que convierte directamente los valores de movilidad electroforética en valores de potencial zeta empleando la ecuación de HelmholtzSmoluchowski. Se midió el potencial zeta en función del pH, para lo cual $40 \mathrm{mg}$ de muestra se dispersaron en $40 \mathrm{~mL}$ de una solución $10^{-3} \mathrm{M} \mathrm{KCl}$ (electrolito inerte); la suspensión se agitó magnéticamente y el $\mathrm{pH}$ de la suspensión se ajustó agregando gotas de $\mathrm{HCl}$ o $\mathrm{KOH}$ de diferentes concentraciones hasta alcanzar el equilibrio (10 min).

\subsubsection{Análisis térmicos}

Las mediciones se llevaron a cabo en un equipo NETZSCH STA 409 PC/PG con alúmina como referencia. Las muestras de $50 \mathrm{mg}$ se colocaron en crisoles de alúmina y fueron calentadas desde 30 hasta $1000^{\circ} \mathrm{C}$ a una rampa de $10{ }^{\circ} \mathrm{C} / \mathrm{min}$ en atmósfera de aire. Los intervalos de temperaturas asociados a cada paso de descomposición térmica se determinaron analizando las curvas Tg y DTg. Se tuvieron en cuenta los puntos de inflexión en la curva $\mathrm{Tg}$, y las temperaturas a las cuales iniciaban y terminaban los picos DTg [Keidar et al., 2015]. 


\subsubsection{Espectroscopía infrarroja}

La espectroscopía infrarroja surge como resultado de la interacción de la materia con radiación electromagnética en el rango de números de onda de 4000 a $400 \mathrm{~cm}^{-1}$ (infrarrojo medio o MIR) y de 12821 a $4000 \mathrm{~cm}^{-1}$ (infrarrojo cercano o NIR). Esta interacción provoca la transición de modos vibracionales y rotacionales de grupos funcionales en su estado electrónico basal a un estado excitado [Workman et al., 2008]. En particular, la radiación MIR provoca transiciones en los modos fundamentales de vibración (estiramiento, flexión, tijereteo) mientras que la radiación NIR provoca sobretonos y combinaciones de los modos fundamentales de vibración, especialmente de estiramiento y flexión.

La frecuencia de radiación MIR absorbida depende tanto de la masa atómica como de la longitud y constante de fuerza de los enlaces interatómicos presentes en la estructura de las arcillas [Heller-Kallai et al., 2006]. Es decir que esta espectroscopía puede utilizarse con fines cualitativos para determinar la presencia de grupos funcionales en una muestra y/o dilucidar el desarrollo de interacciones entre éstos (mediante el corrimiento de sus bandas características hacia menores o mayores números de onda en el espectro). En sistemas como el que aquí se estudia, la técnica MIR fue ampliamente utilizada para identificar los grupos $\mathrm{CH}_{2}, \mathrm{CH}_{3}$ y $\mathrm{NH}_{3}$ provenientes de surfactantes orgánicos en organo-arcillas [Madejová et al., 2011]. En general, la técnica empleada para colectar espectros MIR es la de absorción en discos prensados, en la cual la muestra es dispersada en $\mathrm{KBr}$ en una relación menor al 1\%. En la técnica de absorción se asume que se cumple la Ley de Lambert-Beer, es decir que la absorbancia de una determinada vibración molecular es el producto de su coeficiente de absortividad molar, la concentración de moléculas y el espesor de la pastilla en el haz de medición. Por ello, la espectroscopía MIR podría utilizarse también con fines cuantitativos. En este trabajo, se realizaron análisis cualitativos y semi-cuantitativos tomando como referencia la banda del estiramiento Si-O de la montmorillonita, que es la más intensa en el espectro de las muestras.

La espectroscopia NIR provee información muchas veces no disponible en los espectros MIR, ya que es posible el reconocimiento sin ambigüedad de algunos modos vibracionales que solapan con otras bandas de absorción en MIR (por ejemplo los de $\mathrm{NH}_{2}{ }^{+}, \mathrm{NH}^{+}, \mathrm{CH}(\mathrm{Ar})$ y $\left.\mathrm{CH}_{3}-\mathrm{N}\right)$ [Madejová et al., 2011]. Además, las bandas asociadas a los grupos $\mathrm{OH}$ de las láminas T:O:T (primer sobretono y combinaciones) son muy sensibles a cambios en la estructura del mineral, por lo que cualquier cambio químico que afecte el entorno electrónico de estos grupos son identificables en un espectro NIR. En este caso, la técnica de reflectancia difusa (DRIFT) es especialmente apropiada y no requiere la dilución 
de la muestra. En esta espectroscopía, los análisis cuantitativos o semi-cuantitativos son más difíciles, si no imposibles, debido a la naturaleza de banda ancha de los espectros NIR generada en la superposición de diferentes bandas de sobretono y combinaciones que hacen que no se puedan resolver bien las señales individuales como en MIR.

En este trabajo, los espectros infrarrojos en las regiones MIR y NIR se utilizaron para dilucidar la presencia tanto del ODTMA en la estructura del adsorbente, así como la del fungicida en los productos de adsorción y la manera en que éstos interactúan con la montmorillonita.

Los espectros de FTIR se colectaron empleando un espectrofotómetro Nicolet 6700 de Thermo Scientific. Para medir los espectros MIR y NIR, se utilizaron las técnicas de disco prensado (relación muestra $/ \mathrm{KBr}=1 \mathrm{mg} / 200 \mathrm{mg}$ ) y la técnica de reflectancia difusa, respectivamente. Se obtuvieron los espectros mediante la acumulación de 64 barridos y una resolución de $4 \mathrm{~cm}^{-1}$. El tratamiento de los resultados se llevó a cabo utilizando el paquete de software Thermo Scientific OMNIC, el cual permitió identificar el número de onda de los máximos de los picos y normalizar los espectros respecto de la banda de estiramiento del enlace Si-O para el análisis semi-cuantitativo.

\subsubsection{Carbono orgánico total}

La cuantificación de carbono orgánico total permite conocer el contenido exacto de carbono elemental en un sólido. Se realiza en un analizador elemental que consiste en una cámara de combustión y un detector de infrarrojo. La muestra es colocada dentro de la cámara de combustión a una temperatura de $1000{ }^{\circ} \mathrm{C}$ en presencia de oxígeno, proceso que provoca la liberación de $\mathrm{CO}_{2}$ proveniente de todo el carbono presente en la misma. $\mathrm{El} \mathrm{CO}_{2}$ generado es cuantificado con un detector de infrarrojo.

Las muestras se analizaron en un equipo EMIA 320 V2 AC, HORIBA Jobin Yvon por triplicado. El equipo indicó como resultado un porcentaje de carbono en peso $(\% \mathrm{C})$, a partir del cual se calculó el contenido de ODTMA de los adsorbentes (medidos como porcentajes del valor de la CIC, \%CIC) mediante la Ecuación 3.1:

$$
\text { Contenido de ODTMA }[\% C I C]=\frac{(\% C) \times 1000 \frac{\mathrm{mmol}}{\mathrm{mol}}}{252 \frac{\mathrm{g}(\mathrm{C})}{\operatorname{mol}(\text { ODTMA })} \times 0,825 \frac{\mathrm{mmol}}{\mathrm{g}}}
$$

Los valores que se presentan en la Sección 3.3.1 son el valor promedio de tres muestras.

\subsubsection{Diámetro aparente}

Cuando se hace pasar un haz de luz a través de una suspensión coloidal, éste sufre una dispersión causada por la diferencia en el índice de refracción entre el medio líquido y el 
material sólido. Se forma de esta manera el llamado haz de Tyndall que es proporcional al tamaño de las partículas. Debido a que el método supone partículas esféricas, en el caso de las arcillas (laminares) la determinación otorga un valor de diámetro aparente de la esfera equivalente (DAp), a partir de un cálculo estadístico que el equipo realiza en cada barrido.

En este trabajo se determinó el DAp de los adsorbentes ya que el cambio en la naturaleza eléctrica de la superficie externa de la montmorillonita con el agregado de surfactante permite la formación de aglomerados, y en consecuencia se ve modificada su capacidad de coagulación.

El equipo utilizado fue el mismo que para determinar potencial zeta, pero con la función tamaño de partícula, operando a $\lambda=635 \mathrm{~nm}$ y empleando láser de estado sólido a $15 \mathrm{~mW}$, ángulo de dispersión $=90^{\circ}$ y temperatura $=25^{\circ} \mathrm{C}$. Las determinaciones en este caso se realizaron suspendiendo la muestra en $[\mathrm{KCl}]=10^{-3} \mathrm{M}(1 \% \mathrm{p} / \mathrm{p})$, al $\mathrm{pH}$ natural.

\subsubsection{Estudios de adsorción}

Los estudios de adsorción implican poner en contacto una solución de adsorbato con el adsorbente y luego de transcurrido un dado tiempo de agitación, separar el sólido de la solución y determinar la concentración final del adsorbato en el sobrenadante, mediante alguna técnica de cuantificación. En general, se construyen curvas en las cuales se grafica cantidad adsorbida por unidad de masa en función de algún parámetro (por ejemplo tiempo, concentración del adsorbato en el sobrenadante, $\mathrm{pH}$, fuerza iónica, etc.).

En este trabajo, para separar el sólido de la solución, se centrifugaron las suspensiones a $14.000 \mathrm{rpm}$ durante $15 \mathrm{~min}$. La concentración de IMZ en los sobrenadantes se determinó mediante cromatografía líquida de alta resolución (HPLC) acoplado con un detector UVvisible $(\lambda=220$ y $272 \mathrm{~nm})$. Se usó un equipo Shimadzu con columna C18 (4,6 mm x 250 $\mathrm{mm} ; 4,6 \mu \mathrm{m})$. La fase móvil fue metanol/agua (80/20) fluyendo a $1 \mathrm{~mL} / \mathrm{min}$ y el volumen inyectado $=20 \mu \mathrm{L}$. El intervalo lineal fue $2,5-800 \mathrm{mg} / \mathrm{L}\left(\mathrm{R}^{2}=0,999\right)$ y los límites de detección (LD) y de cuantificación (LC) fueron $\mathrm{LD}=0,117 \mathrm{mg} / \mathrm{L}$ y $\mathrm{LC}=0,321 \mathrm{mg} / \mathrm{L}$ [Suarez Pérez et al., 2009].

Los estudios de la variación de la cantidad adsorbida por unidad de masa $\left(q_{t}[\mathrm{mg} / \mathrm{g}]\right)$ en función del tiempo, se denominan cinéticas de adsorción. $q_{t}$ se calcula según la Ecuación 3.2:

$$
q_{t}=\frac{C_{i}-C_{f}(t)}{m}
$$


donde $C_{i}[\mathrm{mg} / \mathrm{L}]$ y $C_{f}(t)[\mathrm{mg} / \mathrm{L}]$ son las concentraciones inicial $(t=0)$ y final $(t=t)$ de IMZ, y $m[\mathrm{~g} / \mathrm{L}]$ es la relación sólido/solución utilizada. En este trabajo se realizaron cinéticas de adsorción de IMZ en Mt y OMt137 con el objetivo de determinar el tiempo necesario para alcanzar el equilibrio $\left(t_{e q}\right)$. La concentración inicial de fungicida fue $C_{i}=600 \mathrm{mg} / \mathrm{L} \mathrm{y} \mathrm{la}$ relación sólido/solución $=1 \mathrm{mg} / \mathrm{mL}$.

En las isotermas de adsorción, el parámetro que se varía es la concentración inicial de fungicida manteniendo la temperatura fija. Los datos experimentales se expresan graficando la cantidad adsorbida por unidad de masa en el equilibrio ( $Q[\mathrm{mg} / \mathrm{g}]$ ) en función de $C_{f} Q$ se calcula a partir de la Ecuación 3.3:

$$
Q=\frac{C_{i}-C_{f}}{m}
$$

donde $C_{f}$ no depende de $t$, sino que es la concentración final de IMZ determinada a $t=t_{e q}$.

El ajuste de ecuaciones matemáticas a las curvas de $\mathcal{Q}$ vs. $C_{f}$ permite comparar el desempeño de distintos adsorbentes y la afinidad de éstos por un adsorbato. Para hacerlo, es necesario encontrar un modelo que describa correctamente los datos experimentales. Las isotermas de adsorción han sido descritas en muchas formas matemáticas, algunas basadas en un modelo físico de adsorción; otras empíricas, siendo las más comunes las de Langmuir y Freundlich.

La ecuación de Langmuir

$$
Q=\frac{Q_{\max } \cdot k \cdot C_{f}}{\left(1+k \cdot C_{f}\right)}
$$

asume una adsorción monocapa sobre una superficie con un número finito de sitios energéticamente equivalentes y sin interacción entre las moléculas adsorbidas. En la Ecuación 3.4, $Q_{\max }[\mathrm{mg} / \mathrm{g}]$ es la cantidad máxima adsorbida y $k[\mathrm{~L} / \mathrm{mg}]$ es la constante de Langmuir, que se relaciona con la energía de interacción adsorbente-adsorbato.

La ecuación de Freundlich

$$
Q=K_{f} \cdot C_{f}^{1 / n}
$$

es una ecuación empírica basada en la adsorción monocapa sobre una superficie con sitios energéticamente heterogéneos. Supone interacciones entre las moléculas adsorbidas y también asume que existe un número infinito de sitios de reacción desocupados; sin embargo, no muestra una capacidad de adsorción máxima como es el caso de Langmuir. En la Ecuación 3.5, $K_{f}[\mathrm{~L} / \mathrm{g}]$ indica la afinidad del adsorbente por el adsorbato, y $1 / n$ (adimensional) indica cómo varía la adsorción en función de la concentración. 
En este capítulo, se evaluaron estos dos modelos para describir los datos experimentales y analizar el comportamiento de los sistemas en las condiciones estudiadas, comparar el rendimiento y afinidad de los distintos adsorbentes por el fungicida.

Las isotermas se realizaron en tubos de plástico empleando un tiempo de contacto de 24 h a $25{ }^{\circ} \mathrm{C}$ bajo agitación constante. Se realizaron isotermas tipo batch, empleando una relación sólido/solución $=1 \mathrm{~g} / \mathrm{L}$ y un intervalo de concentraciones de IMZ entre 10-600 $\mathrm{mg} / \mathrm{L}$. Los sólidos correspondientes al punto de máxima concentración inicial de IMZ en las isotermas $\left(C_{i}=600 \mathrm{mg} / \mathrm{L}\right)$, luego de separarse de la suspensión mediante centrifugación, se lavaron con agua destilada, se secaron en estufa a $60{ }^{\circ} \mathrm{C}$, se denominaron anteponiendo IMZ- al nombre del adsorbente y se caracterizaron, según lo descripto en la Sección 3.2.2.

La influencia del pH en la adsorción se estudió de distintas formas. En las isotermas, se decidió dejar evolucionar naturalmente el $\mathrm{pH}$ de las suspensiones, sin el agregado de ácido ni de base y se lo midió al comienzo $(t=0)$ y al final $\left(t_{\text {eq }}=24 \mathrm{~h}\right)$, ya que, teniendo en cuenta el carácter básico del fungicida, esto otorgaría información acerca de reacciones ácido-base involucradas en el proceso de adsorción. Se observó que el $\mathrm{pH}$ aumentó con la concentración de equilibrio de IMZ y con el paso del tiempo entre 6,8 y 8,5 para Mt y entre 6,8 y 7,5 para las OMts. Además, se realizó la isoterma de adsorción de Mt manteniendo un $\mathrm{pH}$ constante e igual a 7, ajustándolo con pequeñas alícuotas de $\mathrm{HCl}$ o $\mathrm{KOH}$ de diferentes concentraciones. Por último, se construyeron curvas de eficiencia de remoción ( $E[\%])$ en función del $\mathrm{pH}$ para las muestras Mt, OMt39 y OMt137. La eficiencia de remoción

$$
E=\frac{C_{i}-C_{f}}{C_{i}} \times 100 \%
$$

corresponde al porcentaje de IMZ que se remueve de la fase acuosa durante la adsorción. Se calculó E para los puntos de $C_{i}=600 \mathrm{mg} / \mathrm{L}$, usando una relación sólido/solución $=1$ $\mathrm{g} / \mathrm{L}$ a $25{ }^{\circ} \mathrm{C}$ con agitación durante $24 \mathrm{~h}$.

\subsection{Resultados y discusión}

\subsubsection{Caracterización de los adsorbentes}

En la Tabla 3.1 se muestra el contenido de ODTMA de los adsorbentes (porcentajes respecto de la $\mathrm{CIC}$ ) y su diámetro aparente, mientras que en las Figuras 3.2 y 3.3 , se muestran los difractogramas y curvas de potencial zeta de los mismos, respectivamente. 
Tabla 3.1 Contenido de ODTMA y diámetro aparente de Mt y OMts.

\begin{tabular}{|c|c|c|c|}
\hline Muestra & $\begin{array}{c}\text { ODTMA agregado } \\
{[\% \mathrm{CIC}]}\end{array}$ & $\begin{array}{c}\text { ODTMA adsorbido } \\
{[\% \mathrm{CIC}]}\end{array}$ & $\begin{array}{c}\text { DAp } \\
{[\mathrm{nm}]}\end{array}$ \\
\hline $\mathrm{Mt}$ & n.c. & n.c. & $674 \pm 51$ \\
OMt39 & 39 & 38 & $1730 \pm 32$ \\
OMt55 & 55 & 54 & $2399 \pm 38$ \\
OMt75 & 105 & 74 & $2802 \pm 53$ \\
OMt137 & 211 & 137 & $8351 \pm 46$ \\
\hline
\end{tabular}

n.c.: no correponde
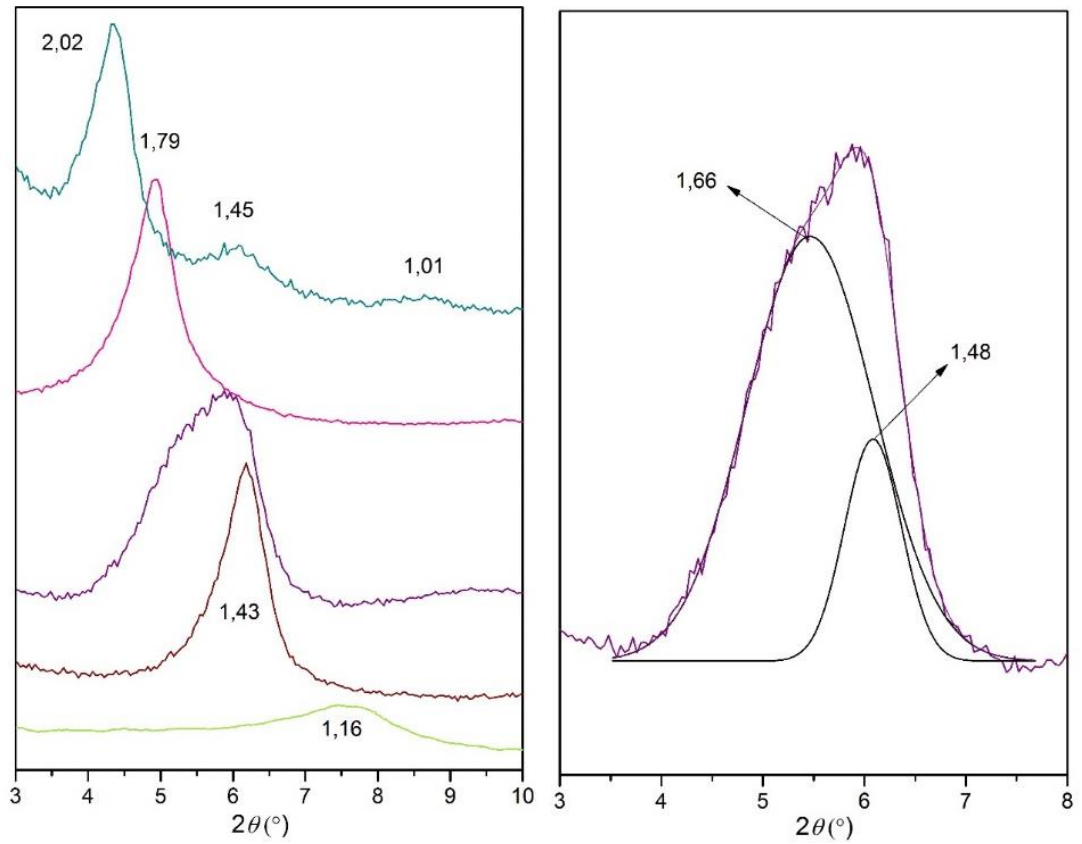

Figura 3.2 Izquierda: difractogramas de $(-) \mathrm{Mt},(-)$ OMt39, (-) OMt55, (-) OMt75 y (-) OMt137. Derecha: descomposición matemática del pico do01 de OMt55. Los valores corresponden al espaciado basal $[\mathrm{nm}]$.

El análisis de carbono total indicó que para las muestras OMt39 y OMt55, todo el ODTMA añadido se unió a la superficie de la montmorillonita, mientras que cuando el agregado de ODTMA fue $105 \%$ CIC, sólo un 75 \%CIC quedó retenido en el sólido. Por último, cuando la cantidad de surfactante superó el $200 \%$ CIC, apenas un 137 \%CIC quedó en la estructura de la organo-montmorillonita (muestra OMt137).

Xu y Boyd [1995] propusieron un modelo para explicar la adsorción de una amina cuaternaria con una cadena de 16 átomos de carbono (hexadeciltrimetil amonio, HDTMA) en una esméctica sódica. Demostraron que, cuando el porcentaje de surfactante en la 
organo-montmorillonita respecto de la CIC era menor al 75\%, la adsorción del mismo ocurría a través del intercambio catiónico con los cationes interlaminares (interacciones electrostáticas entre los cationes orgánicos y la superficie negativa de la montmorillonita); mientras que cuando el contenido de surfactante excedía ese valor, a las interacciones electrostáticas HDTMA-esmectita se le sumaban interacciones tipo van der Waals entre las cadenas hidrocarbonadas del surfactante.

En las muestras estudiadas, se observó que cuando el contenido de ODTMA fue menor a $75 \%$ CIC, la adsorción del surfactante ocurrió principalmente en los sitios de carga permanente de la Mt, es decir, la fuerza impulsora de la adsorción fue el intercambio catiónico. Esto se tradujo en un incremento del espaciado basal de 1,16 nm en Mt hasta $1,79 \mathrm{~nm}$ en OMt75 (Figura 3.2). La muestra OMt39 presentó un espaciado basal de 1,43 $\mathrm{nm}$, asignado a la disposición tipo monocapa del surfactante [Brindley y Moll, 1965]. Para la muestra OMt55, se observó un ensanchamiento del pico cuya deconvolución matemática indicó dos espaciados (1,66 y 1,48 nm) que fueron atribuidos a la presencia de dos tipos de arreglo en la intercapa del surfactante (monocapa y bicapa, respectivamente). El pico de reflexión observado para la muestra OMt75 indicó un espaciado de 1,79 nm, que se asignó a la disposición lateral del surfactante en forma de bicapa.

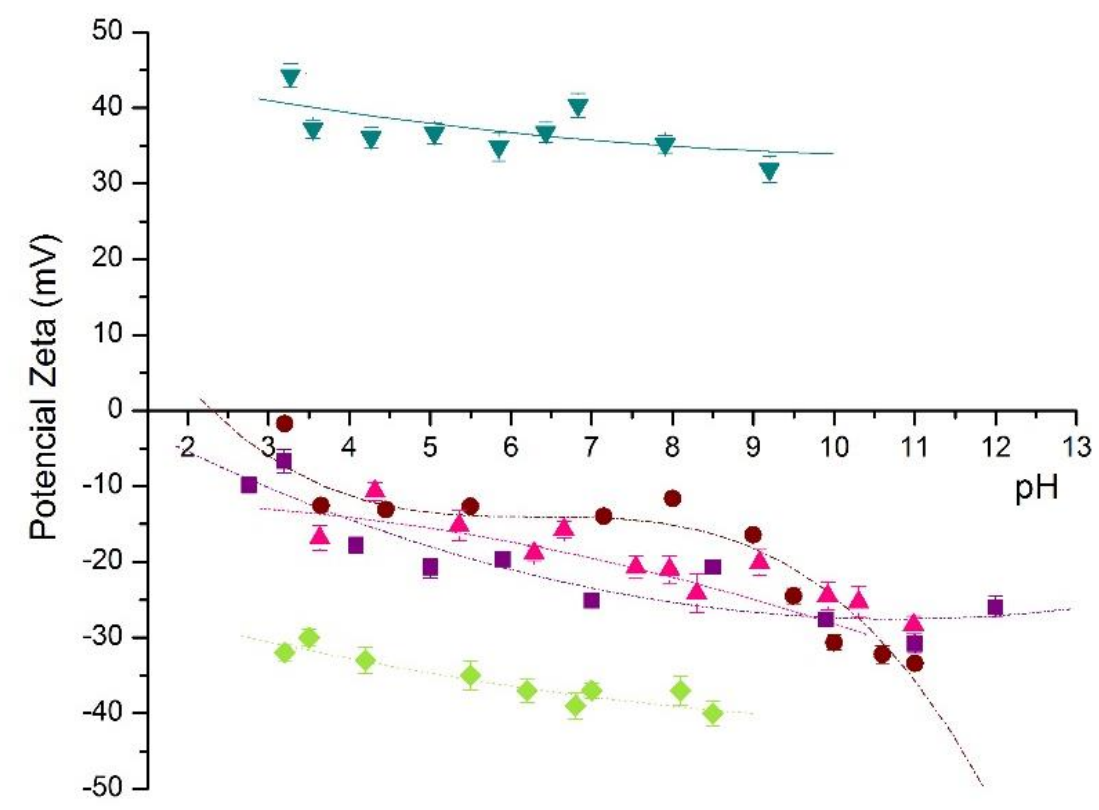

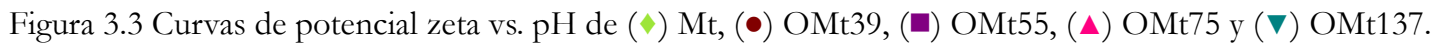
Las curvas son regresiones lineales utilizando una ecuación polinomial, con el objetivo de facilitar el seguimiento de los puntos correspondientes a cada muestra. 
Sin embargo, el cambio en la carga superficial de los adsorbentes, no presentó una tendencia tan clara. Como puede verse en la Figura 3.3, el incremento de contenido de ODTMA hasta $75 \%$ CIC provocó una disminución de aproximadamente $20 \mathrm{mV}$ de la carga superficial negativa respecto a la observada en $\mathrm{Mt}$ (alrededor de $-30 \mathrm{mV}$ en todo el intervalo de $\mathrm{pH}$ estudiado, originado en las sustituciones isomórficas y en que las cargas de borde dependientes del $\mathrm{pH}$ corresponden a menos de un 1\% de la carga total, como se explicó en la Sección 2.1.2) pero no hubo diferencias significativas entre las muestras. Esto sugirió que sólo una pequeña cantidad de ODTMA se distribuyó en los sitios de superficie externa de las muestras OMt39, OMt55 y OMt75.

El tamaño de los agregados, medidos como DAp (Tabla 3.1), incrementó su valor en cuatro veces para OMt39, OMt55 y OMt75 respecto de Mt. Probablemente, la exposición de las colas alquílicas hidrofóbicas a la fase acuosa desestabiliza el complejo ODTMAmontmorillonita y como consecuencia, se forman agregados más voluminosos en los cuales interactúan las cadenas alquílicas de ODTMA presentes en la superficie externa de las organo-montmorillonitas, disminuyendo así el contacto entre las cadenas hidrofóbicas y el agua.

Los estudios de DRX y potencial zeta de la muestra OMt137 (Figuras 3.2 y 3.3), mostraron que el ODTMA en este caso, se distribuyó tanto en los sitios de carga permanente como en los sitios de superficie externa. Por DRX, se observó la formación de un sistema interlaminar heterogéneo, con tres espaciados basales: 2,02; 1,45 y 1,01 nm. El primero, se asignó a un arreglo tipo parafina o de pseudo-tricapa de las cadenas alquílicas de ODTMA [Zhu et al., 2003]; el segundo se atribuyó a un arreglo tipo monocapa de las mismas; mientras que el último, con valor menor al espaciado de la Mt, pudo haberse originado en el colapso de algunas láminas debido a la remoción de los cationes inorgánicos y sus respectivas esferas de hidratación por la entrada del surfactante durante la intercalación [Baldassari et al., 2006; Bianchi et al., 2013]. Esta muestra presentó, a diferencia de las analizadas anteriormente, carga superficial positiva en todo el intervalo de $\mathrm{pH}$ estudiado (Figura 3.3), indicando que cuando el contenido de ODTMA supera el 100 \%CIC, el exceso de ODTMA cubre los sitios de superficie externa y los sitios de borde de la montmorillonita [Pecini y Avena, 2013]. El DAp de esta muestra, 8350 nm, duplicó el valor de las OMts con menor contenido de ODTMA. Este valor tan alto de tamaño de partícula indicó que se desarrollan fuerzas de van der Waals atractivas entre las cadenas alquílicas de la superficie externa, con lo cual se forman aglomerados que minimizan el contacto de estas colas hidrofóbicas con el agua. Energéticamente, estas fuerzas son 
mayores que las fuerzas electrostáticas repulsivas de los cationes que provocarían la delaminación de la OMt y un aumento de la dispersión de las partículas en la suspensión y, consecuentemente, un incremento en su turbidez [Xu y Boyd, 1995]. En el Anexo 1 (Figura A1), se muestra la coagulación en agua de organo-montmorillonitas con diferentes surfactantes, donde se refleja la diferencia de comportamiento respecto a Mt.

En las Figura 3.4 se muestran las curvas ATD, Tg y DTg obtenidas para Mt y el surfactante puro, mientras que las temperaturas que definen las regiones y pasos de descomposición térmica de todas las muestras, junto a las pérdidas de masa registradas por Tg y los picos DTg y ATD se organizan en la Tabla 3.2.

Tal como se describió en el Capítulo 2, la montmorillonita presentó en ATD en la Región I $\left(25-145{ }^{\circ} \mathrm{C}\right)$ un importante pico endotérmico a $77{ }^{\circ} \mathrm{C}$ correspondiente a la desorción de agua fisisorbida, asociado a un pico DTg a $74{ }^{\circ} \mathrm{C}$. En este intervalo se vio además un pico endotérmico pequeño a $\operatorname{los} 112{ }^{\circ} \mathrm{C}$, con un pico DTg asociado a los 108 ${ }^{\circ} \mathrm{C}$. En conjunto, ambos procesos condujeron a la pérdida de un $11,5 \%$ de la masa inicial de la muestra.

El pico DTg asociado al proceso de deshidroxilación comenzó a los $485^{\circ} \mathrm{C}$ y terminó a los $758{ }^{\circ} \mathrm{C}$ para esta muestra (Región III), donde también se registró un pico endotérmico en ATD a los $658^{\circ} \mathrm{C}$ con una pérdida de masa de 3,3\%.

Aunque en la Región II no se observaron picos ATD ni DTg se registró en Tg una pérdida de masa del 0,5\%, evidenciando que la deshidratación continúa en esta región.
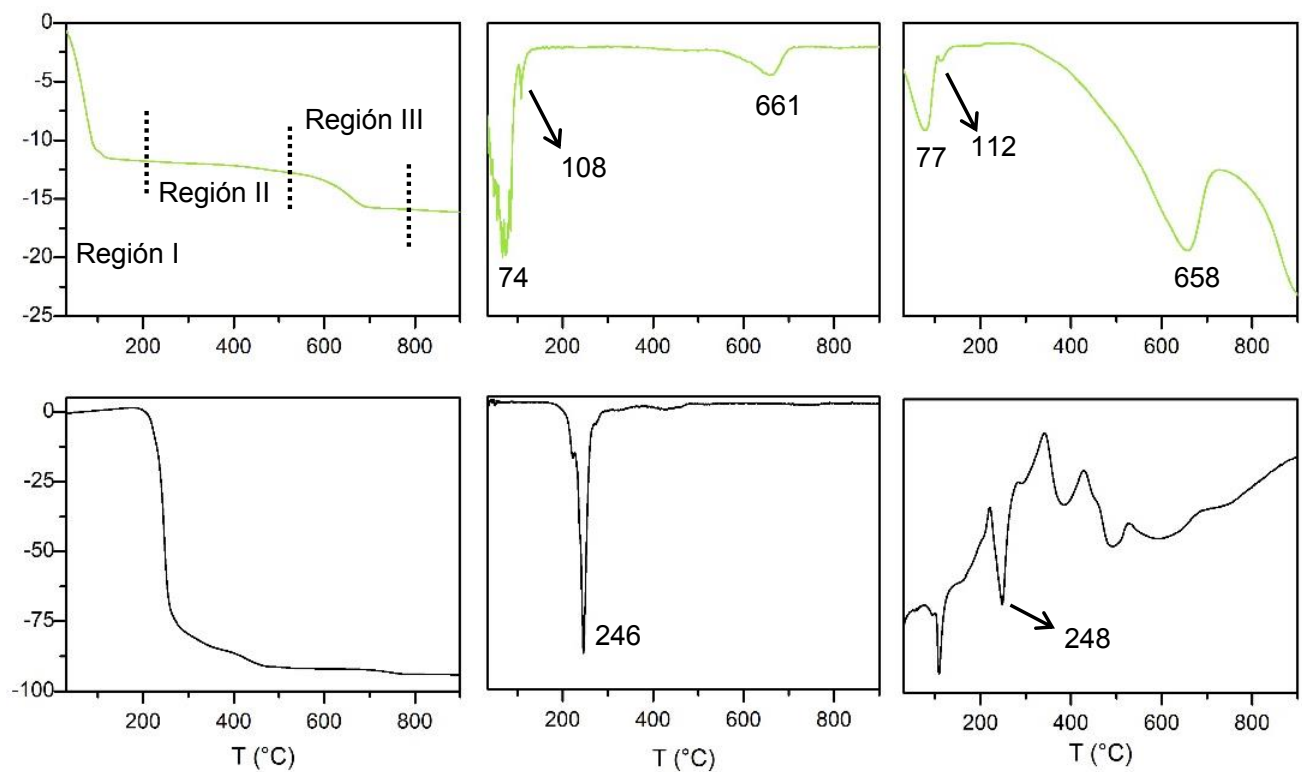

Figura 3.4 Curvas de análisis térmicos. De izquierda a derecha: Tg, DTg y ATD de $(-)$ Mt y (-) ODTMA. 
El surfactante puro (Figura 3.4), se descompuso en distintas etapas como puede evidenciarse de la curva de ATD, consistente en diversos picos endo- y exotérmicos. El salto más importante de pérdida de masa condujo a un pico DTg centrado a $246{ }^{\circ} \mathrm{C}$. Este pico se correspondió con un pico ATD endotérmico a los $248{ }^{\circ} \mathrm{C}$. Este proceso conllevó a la pérdida del $81 \%$ de la masa, aunque la descomposición continúo a temperaturas mayores (nótese que a los $600{ }^{\circ} \mathrm{C}$ la pérdida de masa es del 92\%). El pico endotérmico se asignó a la transición de fase de estado sólido a líquido, seguida de reacciones de oxidación y posterior pirólisis de los productos (procesos exotérmicos) [Keidar et al., 2015].

El comportamiento térmico de las organo-montmorillonitas fue diferente tanto al de la Mt como al del ODTMA, confirmando la interacción entre el mineral y la amina. Dentro de las Regiones I, III y particularmente de la Región II, pueden observarse distintos picos en el ATD y en el DTg correspondientes a distintos pasos de descomposición, consecuencia de distintas reacciones térmicas (Figura 3.5, Tabla 3.2).

En la Región I, la disminución de porcentaje de pérdida de masa desde OMt39 a OMt137 (menor en todos los casos a Mt) se debe a la disminución del contenido de agua atribuido al incremento de hidrofobicidad de las muestras (Tabla 3.2) y a la disminución de cationes $\mathrm{Na}^{+}$interlaminares con sus correspondientes esferas de hidratación, al aumentar el contenido de ODTMA. El pico DTg asociado al último proceso no se vio en las muestras, mientras que el pico DTg asociado a la deshidratación de agua fisisorbida disminuyó su temperatura indicando la mayor labilidad de las moléculas de agua con el incremento del contenido de ODTMA en las muestras.

Al igual que en el surfactante puro, la descomposición del contenido orgánico en las organo-montmorillonitas (Región II, Figura 3.5, Tabla 3.2) ocurrió en varias etapas. Sin embargo, es importante notar varias diferencias. En todas las muestras, el pico más importante en el DTg en esta región se ubicó a temperaturas más altas que el pico más importante de descomposición del surfactante puro $\left(246{ }^{\circ} \mathrm{C}\right.$, Tabla 3.2). Esto evidencia la interacción entre el ODTMA y la capa siloxánica de la Mt [Hedley et al., 2007]. Al mismo tiempo, esta temperatura disminuyó al incrementar el contenido orgánico. Por ejemplo, el pico DTg de la muestra OMt137 se ubicó $20{ }^{\circ} \mathrm{C}$ más abajo que en OMt75. Esto puede explicarse porque hasta 80\% CIC de intercambio, la interacción entre la amina y la Mt es electrostática, mientras que a porcentajes mayores (como OMt137), aparecen algunas moléculas que se unen como par iónico a la superficie de la muestra a través de interacciones van der Waals entre las cadenas alquílicas, explicando la menor energía -y en consecuencia, menor temperatura- que se necesita para descomponerlas. 
Otra diferencia importante es que el pico ATD endotérmico observado en el surfactante puro no se observa en las muestras intercambiadas. Por el contrario, en todas las organomontmorillonitas, el pico DTg estuvo asociado a picos exotérmicos en el ATD en la Región II. Esto pone de manifiesto que la transición de fase no ocurre cuando la amina se encuentra unida a la montmorillonita y otras reacciones térmicas son las que tienen lugar.

Keidar et al. [2015] diferencia tres pasos de combustión de la materia orgánica cuando se trabaja en atmósfera oxidante (Tabla 3.2). El primer paso corresponde al rango de temperatura $250-380{ }^{\circ} \mathrm{C}$, donde ocurre la oxidación de los átomos de hidrógeno de la materia orgánica a agua (que se desprende en forma gaseosa) y los átomos de $\mathrm{C}$ y $\mathrm{N}$ empiezan a oxidarse. Si la cantidad de materia orgánica es baja en relación a la disponibilidad de oxígeno, la oxidación de $\mathrm{H}, \mathrm{C}$ y $\mathrm{N}$ se completa a relativamente bajas temperaturas; mientras que si la cantidad de compuestos orgánicos es alta, el oxígeno disponible puede no ser suficiente para completar la combustión y por lo tanto además de $\mathrm{H}_{2} \mathrm{O}, \mathrm{CO}_{2}$ y $\mathrm{NO}_{2}$ se formará carbón no-volátil, intercalado entre las láminas de arcilla. Durante el segundo paso de oxidación $\left(380-520^{\circ} \mathrm{C}\right)$, el carbón estable a bajas temperaturas se oxida a $\mathrm{CO}_{2}$ y $\mathrm{NO}_{2}$, generando picos en DTg y ATD y puede dar lugar a otro tipo de carbón, estable a altas temperaturas donde los átomos de carbono penetran en las cavidades hexagonales de la capa siloxánica. La temperatura a la cual comienza este paso depende, entre otras cosas, de la composición, tamaño y forma de la molécula original; y del enlace entre la arcilla y el compuesto orgánico. A temperaturas superiores a $520{ }^{\circ} \mathrm{C}$, ocurre el tercer paso de oxidación de materia orgánica, donde el carbón intercalado se oxida gradualmente a $\mathrm{CO}_{2}$ y $\mathrm{NO}_{2}$, observándose picos alrededor de $750{ }^{\circ} \mathrm{C}$ en DTg y pérdidas de masa hasta $900{ }^{\circ} \mathrm{C}$, los cuales se superponen con el pico de deshidroxilación de la arcilla. El desarrollo de este tercer paso de oxidación depende de que el carbón penetre o no en las cavidades ditrigonales de las láminas siloxánicas, y su aparición como pico exotérmico en ATD también depende del contenido orgánico en la muestra. Si el contenido es bajo, el pico endotérmico de deshidroxilación se superpone y no se puede distinguir el pico asociado a la oxidación del carbón.

La muestra OMt39 no presentó picos a temperaturas mayores a $380{ }^{\circ} \mathrm{C}$ (Figura 3.5). Sin embargo, el hecho de que la pérdida de masa haya duplicado la pérdida de masa de Mt en la Región III (Tabla 3.2) significa que, aun habiendo un bajo contenido de ODTMA, la oxidación en el primer paso ocurre con formación de carbón que se termina de oxidar en dicha región. A medida que el contenido de ODTMA aumentó, además de crecer el porcentaje de pérdida de masa, en las Regiones II y III aparecieron nuevas componentes en 

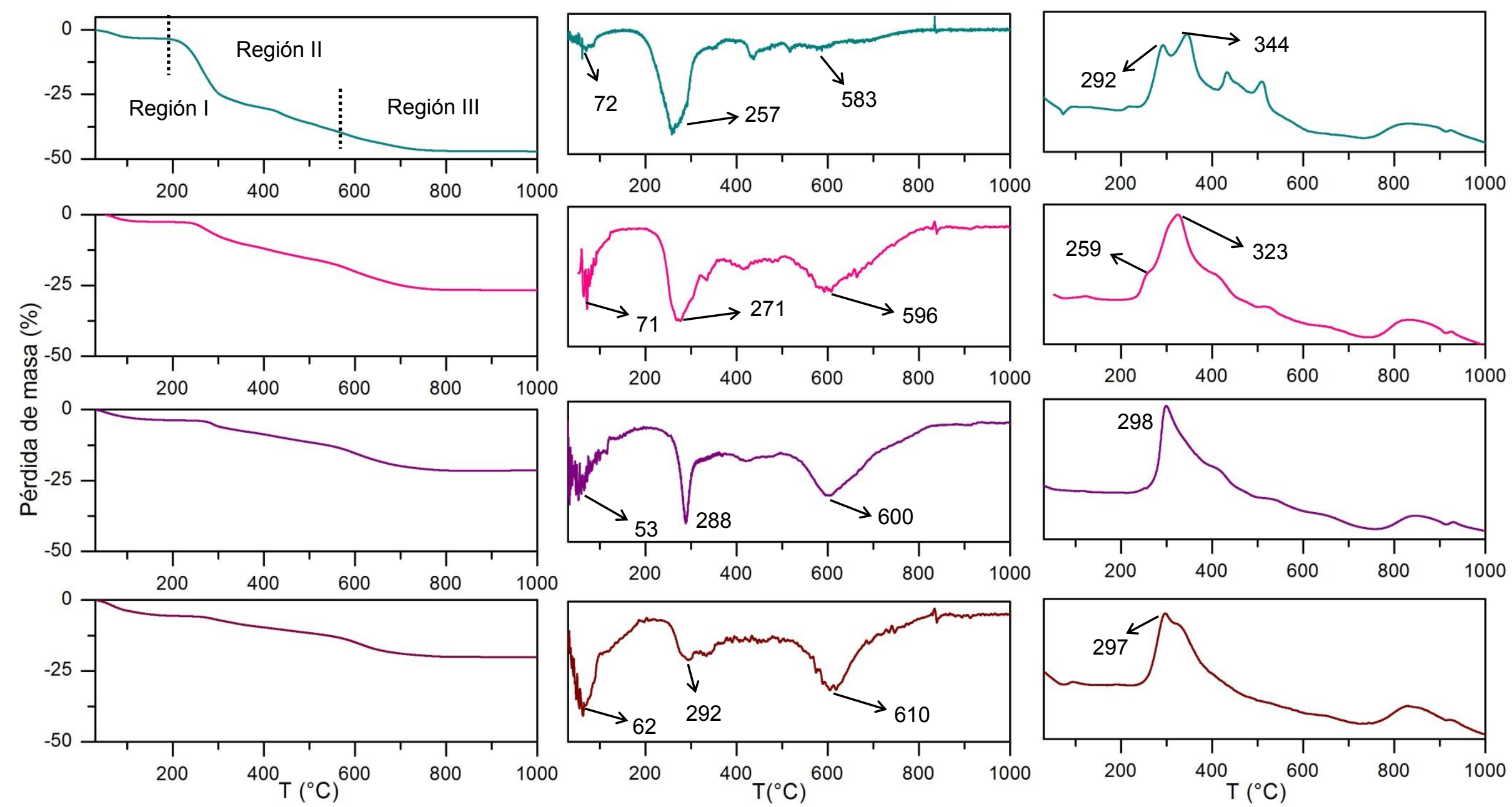

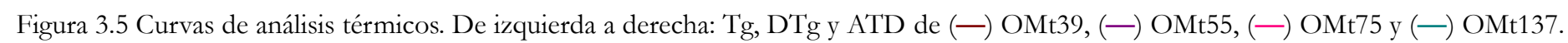


Tabla 3.2 Reacciones térmicas, picos DTg y ATD $\left[{ }^{\circ} \mathrm{C}\right]$ y porcentajes de pérdida de masa [\%] de Mt y OMts.

\begin{tabular}{|c|c|c|c|c|c|c|c|}
\hline & & & Mt & OMt39 & OMt55 & $\mathrm{OMt} 75$ & OMt137 \\
\hline \multirow[t]{6}{*}{ Región I } & \multicolumn{2}{|c|}{ Intervalo de $\mathrm{T}$} & $30-145$ & $30-187$ & $30-208$ & $30-192$ & $30-147$ \\
\hline & \multirow[t]{2}{*}{ Desorción de agua fisisorbida } & DTg & 74 & 62 & 53 & 71 & 72 \\
\hline & & ATD (endo) & 77 & 74 & n.d. & 79 & 73 \\
\hline & \multirow[t]{2}{*}{ Deshidratación de cationes interlaminares } & DTg & 107 & n.d. & n.d. & n.d. & n.c. \\
\hline & & ATD (endo) & 112 & n.d. & n.d. & n.d. & n.c. \\
\hline & Pérdida de masa total & $\operatorname{Tg}$ & 11,5 & 5,4 & 3,7 & 2,6 & 3,2 \\
\hline \multirow[t]{7}{*}{ Región II } & \multicolumn{2}{|c|}{ Intervalo de $\mathrm{T}$} & n.c. & $241-380$ & 208-379 & $192-366$ & $147-384$ \\
\hline & \multirow[t]{2}{*}{ 1er Paso de oxidación de contenido orgánico. } & DTg & n.c. & $292 ; 332$ & 288 & $271 ; 334$ & $257 ; 349$ \\
\hline & & ATD (exo) & n.c. & $297 ; 324$ & 298 & 259 (h); 323 & $292 ; 344$ \\
\hline & \multicolumn{2}{|c|}{ Intervalo de $\mathrm{T}$} & n.c. & n.d. & $379-513$ & $366-505$ & $384-542$ \\
\hline & \multirow[t]{2}{*}{ 2do Paso de oxidación de contenido orgánico. } & DTg & n.c. & n.d. & 419; $464(h)$ & $414 ; 484$ & $437 ; 516$ \\
\hline & & ATD (exo) & n.c. & n.d. & 411 & $407 ; 470(\mathrm{~h})$ & $432 ; 509$ \\
\hline & Pérdida de masa total & $\operatorname{Tg}$ & 0,5 & 6,4 & 8,1 & 12,4 & 35,2 \\
\hline \multirow[t]{6}{*}{ Región III } & \multicolumn{2}{|c|}{ Intervalo de $\mathrm{T}$} & $345-758$ & $523-841$ & $513-862$ & $505-830$ & $542-817$ \\
\hline & \multirow[t]{2}{*}{ Deshidroxilación } & DTg & 661 & 610 & 600 & 596 & 583 \\
\hline & & ATD (endo) & 658 & 609 & n.d. & n.d. & 608 \\
\hline & 3er Paso de oxidación de contenido orgánico & DTg & n.c. & n.d. & n.d. & $664(\mathrm{~h})$ & 676 \\
\hline & (Oxidación de restos carbónicos) & ATD (exo) & n.c. & n.d. & n.d. & 656 & 671 \\
\hline & Pérdida de masa total & $\operatorname{Tg}$ & 3,3 & 7,9 & 9,7 & 10,9 & 10,4 \\
\hline
\end{tabular}

n.c.: no corresponde; n.d.: no determinado; (h): hombro 
las curvas DTg y ATD a temperaturas mayores a $380{ }^{\circ} \mathrm{C}$. Se tratan de procesos exotérmicos probablemente vinculados con el desarrollo de distintos tipos de carbones descriptos por Keidar et al. [2015]. Otra evidencia que apoya este modelo es el hecho de que en la Región III el pico de deshidroxilación se corrió hacia menores temperaturas (Tabla 3.2) lo cual ocurre cuando el carbón penetra en las cavidades ditrigonales de la Mt [Keidar et al., 2015].

El espectro MIR de la Mt se muestra en la Figura 3.6, donde se observan las bandas de vibración de estiramiento $(\nu)$ y flexión $(\delta)$ características de sus grupos estructurales: $\downarrow \mathrm{OH}$ a $3630 \mathrm{~cm}^{-1}$; $\nu \mathrm{SiO}$ a $1045 \mathrm{~cm}^{-1} ; \delta \mathrm{AlAlOH}$ a $918 \mathrm{~cm}^{-1} ; \delta \mathrm{AlMgOH}$ a $844 \mathrm{~cm}^{-1} ; \delta \mathrm{AlOSi}$ a 525 $\mathrm{cm}^{-1}$; $\delta$ SiOSi a $460 \mathrm{~cm}^{-1}$. Las organo-montmorillonitas presentaron en las regiones del espectro MIR mostradas en las Figuras 3.6, bandas de estiramiento y flexión propias de los grupos C-H de ODTMA. Las bandas de absorción alrededor de 2919 y $2850 \mathrm{~cm}^{-1}$ corresponden al estiramiento antisimétrico y simétrico de los grupos $\mathrm{CH}_{2}$, respectivamente. La intensidad de estas bandas aumentó con el aumento de contenido de ODTMA desde OMt39 a OMt137, mientras que su posición (número de onda) disminuyó desde 2927 y $2855 \mathrm{~cm}^{-1}$ para OMt39, hasta 2919 y $2850 \mathrm{~cm}^{-1}$ para OMt137, debido al aumento en la densidad del empaquetamiento de las cadenas alquílicas en la zona interlaminar y consecuente adopción de un arreglo más ordenado [Osman et al., 2004; Jiang et al., 2009]. La aparición de las bandas vibracionales de flexión simétrico $\left(\delta_{\mathrm{s}}\right)$ y antisimétrico $\left(\delta_{\mathrm{as}}\right)$ de las cadenas alquílicas $\left(\delta_{\mathrm{as}} \mathrm{CH}_{3}-\mathrm{N}\right.$ a $1488 \mathrm{~cm}^{-1} ; \delta_{\mathrm{as}} \mathrm{CH}_{2}$ a $1470 \mathrm{~cm}-1 ; \delta_{\mathrm{as}} \mathrm{CH}$ a $1417 \mathrm{~cm}^{-1}$ y $\delta_{\mathrm{s}} \mathrm{CH}_{3}$ a $1383 \mathrm{~cm}^{-1}$ ) también confirmaron la intercalación de los cationes ODTMA en las muestras. La banda alrededor de $1625 \mathrm{~cm}^{-1}$ corresponde a los modos vibracionales de flexión de los grupos $\mathrm{OH}$ de moléculas de agua de las esferas de hidratación de los cationes inorgánicos interlaminares; su intensidad disminuyó a medida que el contenido de ODTMA aumentó en las muestras [Tong et al., 2010]. En la Tabla A1 en el Anexo 1 se detalla la asignación de todas las bandas presentes en los espectros de las muestras correspondientes.

Los espectros NIR de todos los adsorbentes se muestran en la Figura 3.7, mientras que los modos vibracionales a los cuales se asignan las bandas se resumen en la Tabla A2 en el Anexo 1. Todas las muestras presentaron bandas intensas a $\sim 7080$ y $5250 \mathrm{~cm}^{-1}$. La primera fue asignada a contribuciones superpuestas del primer sobretono del estiramiento de grupos hidroxilos estructurales y de moléculas de agua fisisorbidas a la superficie siloxánica $(2 \imath \mathrm{OH})$; mientras que la segunda fue atribuida a $(v+\delta) \mathrm{H}_{2} \mathrm{O}$ [Bishop et al., 1994; Madejová y Komadel, 2001]. El hombro cerca de $6800 \mathrm{~cm}^{-1}$, mejor resuelto para la Mt que para las 
OMts, se atribuyó al primer sobretono del estiramiento $\mathrm{OH}$ de moléculas de agua fuertemente unidas a las capas siloxánicas a través de uniones hidrógeno [Madejová et al., 2011]. La entrada en la zona interlaminar de cationes ODTMA produjo la aparición de nuevas bandas asociadas a los sobretonos de los estiramientos $\mathrm{C}-\mathrm{H}$ en $\mathrm{CH}_{2}$ y $\mathrm{CH}_{3}-\mathrm{N}$ $\left(6200-5300 \mathrm{~cm}^{-1}\right)$ y combinaciones estiramiento/flexión $\left(4800-4000 \mathrm{~cm}^{-1}\right)$.
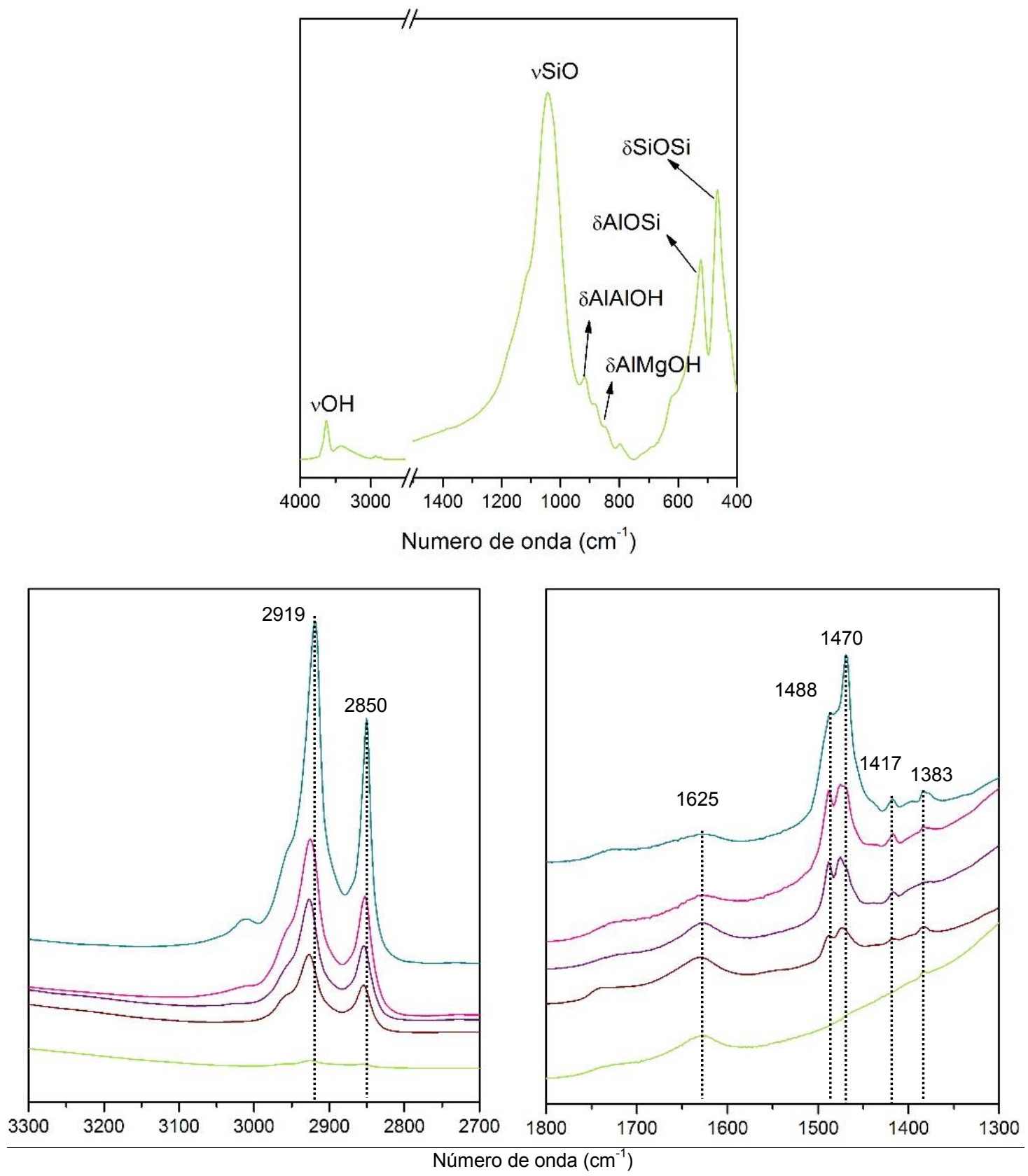

Figura 3.6 Espectros MIR de $(-)$ Mt, $(\longrightarrow)$ OMt39, ( $(-)$ OMt55, ( $(-)$ OMt75 y $(-)$ OMt137. 


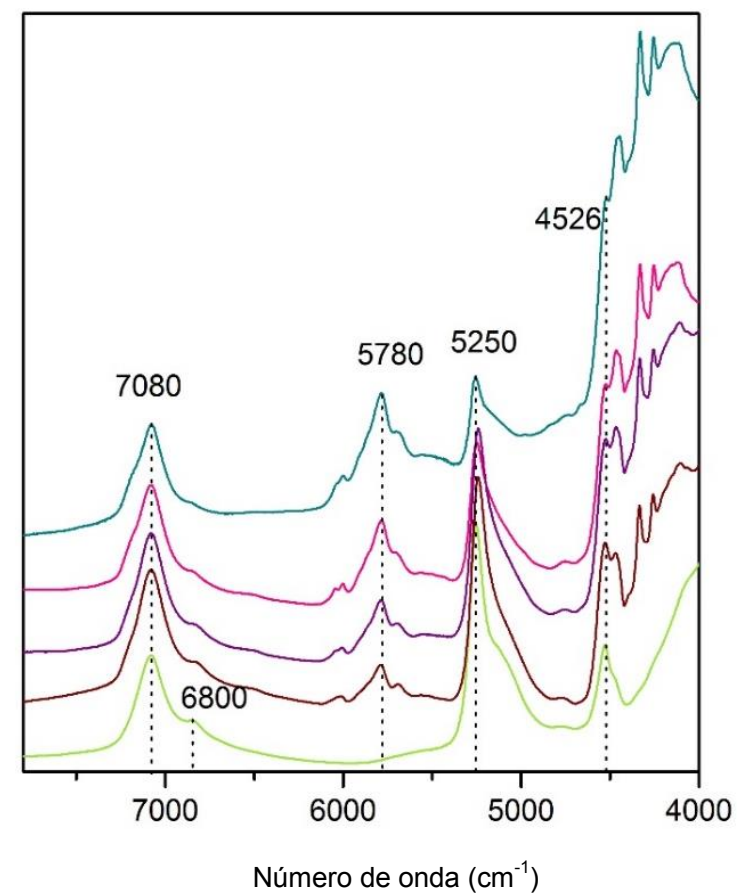

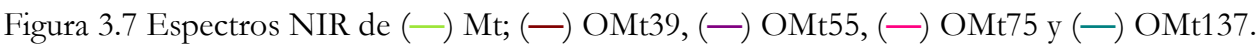

\subsubsection{Estudios de adsorción}

Se construyeron gráficos de adsorción de IMZ en función del tiempo para definir los tiempos necesarios para alcanzar el equilibrio. Los gráficos $q_{t}$ vs. $t$ para Mt y OMt137 se muestran en la Figura 3.8, donde se infiere que la concentración se mantiene prácticamente constante desde los 60 min para Mt y desde los 360 min para OMt137. Sin embargo, considerando que las plantas empacadoras descargan efluentes cada 24 h (1440 min), este último fue el tiempo elegido como tiempo de contacto para ambas muestras

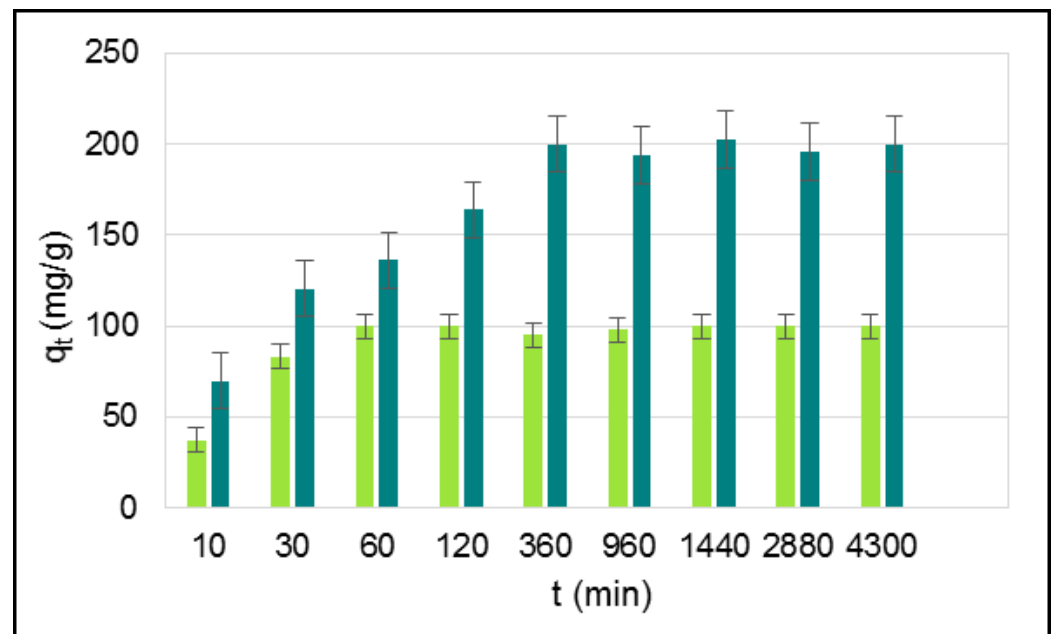

Figura 3.8 Influencia del tiempo en la adsorción de IMZ $\left(C_{i}=600 \mathrm{mg} / \mathrm{L}\right)$ en ( () Mt y (匹) OMt137. 
Las isotermas de adsorción obtenidas para todas las muestras, junto a los correspondientes ajustes de Freundlich y Langmuir, se muestran en la Figura 3.9. Se compararon las isotermas de IMZ en Mt con y sin ajuste de $\mathrm{pH}$ a 7, mientras que las isotermas de IMZ en las OMts se obtuvieron sin ajuste del mismo.

El coeficiente de correlación $\mathrm{R}^{2}$, junto a los parámetros de los modelos de Freundlich y Langmuir, se muestran en la Tabla 3.4. De acuerdo a $\mathrm{R}^{2}$, los datos experimentales pueden ser correctamente descriptos mediante ambos modelos.
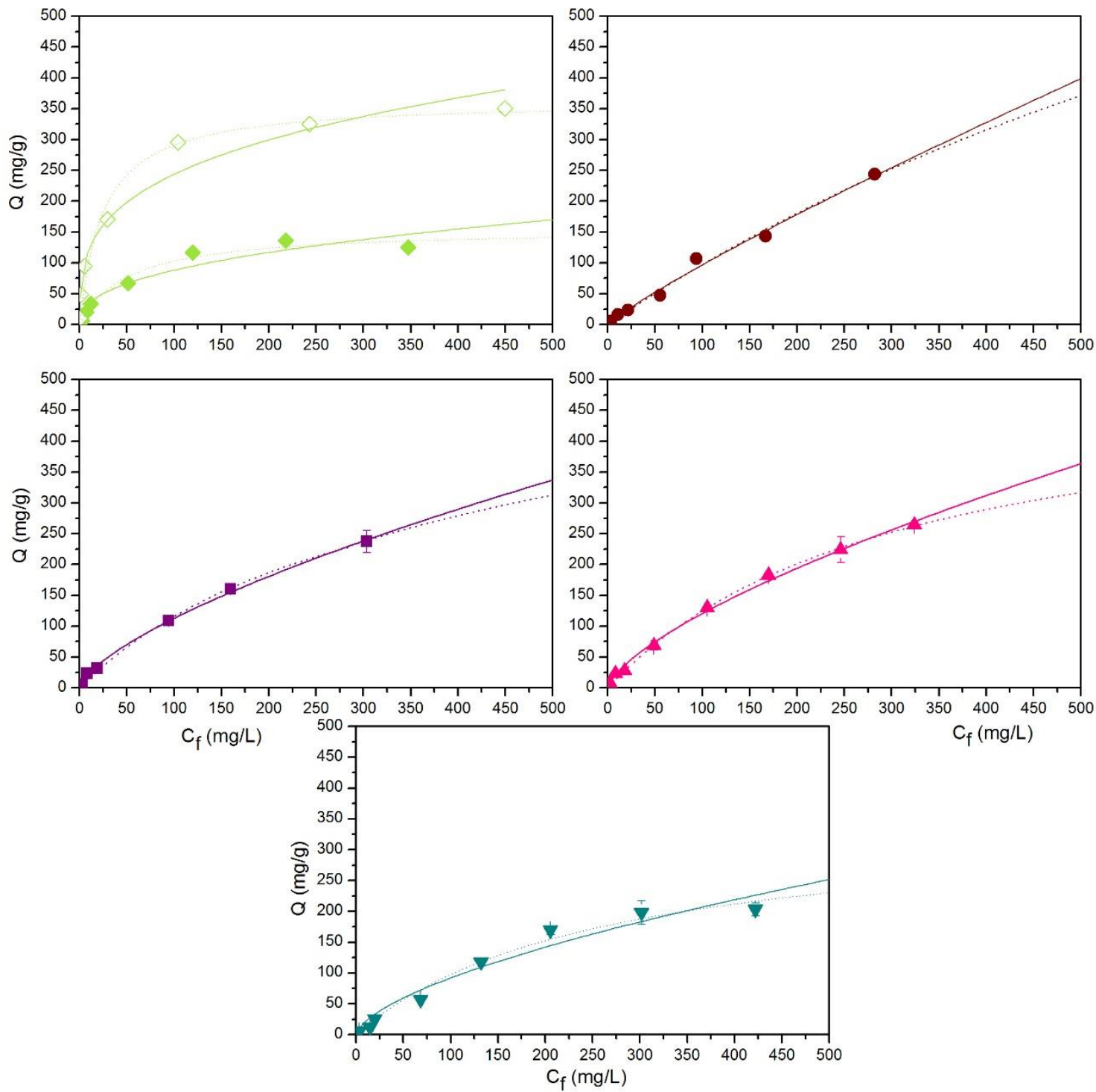

Figura 3.9 Isotermas de adsorción de IMZ en $(\diamond)$ Mt sin ajuste de $\mathrm{pH},(\diamond)$ Mt con pH = 7, (•) OMt39, OMt55, ( $\mathbf{\Delta})$ OMt75 y ( $\mathbf{\nabla})$ OMt137. Las líneas sólidas corresponden a Langmuir y las punteadas a Freundlich.

Si se analizan con profundidad los resultados (Figura 3.9, Tabla 3.3), es posible diferenciar dos comportamientos en las isotermas. Cuando $C_{f}$ fue menor a $50 \mathrm{mg} / \mathrm{L}$, la muestra Mt presentó mejor desempeño como adsorbente (mayores Q) que se reflejó en una 
constante de afinidad más grande respecto al de las muestras OMts. En contraste, para el intervalo de $C_{f}$ entre $50-600 \mathrm{mg} / \mathrm{L}$, las muestras OMts presentaron mejor desempeño que la $\mathrm{Mt}$ sin ajuste de $\mathrm{pH}$, alcanzando mayores valores de $Q_{\max }$ (no hay que perder de vista que $Q_{\max }$ es una extrapolación ya que no siempre las muestras alcanzaron el plateau que identifica la saturación de los sitios de adsorción).

Tabla 3.3 Parámetros obtenidos mediante el ajuste de Freundlich y Langmuir a las isotermas de adsorción de IMZ en Mt y OMts.

\begin{tabular}{|l|c|c|c|c|c|c|}
\hline & \multicolumn{3}{|c|}{ Freundlich } & \multicolumn{3}{c|}{ Langmuir } \\
\hline Isoterma & $K_{F}[\mathrm{~L} / \mathrm{g}]$ & $1 / n$ & $\mathrm{R}^{2}$ & $Q_{\max }[\mathrm{mg} / \mathrm{g}]$ & $k\left(10^{-3}\right)[\mathrm{L} / \mathrm{mg}]$ & $\mathrm{R}^{2}$ \\
\hline Mt & $14 \pm 2$ & $0,40 \pm 0,01$ & 0,925 & $(15 \pm 1) \cdot 10^{1}$ & $20 \pm 5$ & 0,976 \\
Mt $(\mathrm{pH}=7)$ & $62 \pm 5$ & $0,30 \pm 0,04$ & 0,959 & $(36 \pm 5) \cdot 10^{1}$ & $40 \pm 1$ & 0,978 \\
OMt39 & $1,7 \pm 0,8$ & $0,88 \pm 0,06$ & 0,990 & $(13 \pm 3) \cdot 10^{2}$ & $0,84 \pm 0,5$ & 0,983 \\
OMt55 & $4,9 \pm 0,5$ & $0,68 \pm 0,03$ & 0,998 & $(60 \pm 1) \cdot 10^{1}$ & $2,2 \pm 0,8$ & 0,982 \\
OMt75 & $5,1 \pm 0,7$ & $0,69 \pm 0,07$ & 0,991 & $(51 \pm 2) \cdot 10^{1}$ & $3,2 \pm 0,3$ & 0,998 \\
OMt137 & $5,1 \pm 0,9$ & $0,40 \pm 0,05$ & 0,960 & $(35 \pm 2) \cdot 10^{1}$ & $3,9 \pm 0,8$ & 0,991 \\
\hline
\end{tabular}

Socias-Viciana et al. [1998] encontraron una dependencia similar de la concentración en la adsorción del herbicida prometrone en la esmectita Wyoming de Clays Minerals Repository (Clay Minerals Society, Minnesota). Sus resultados fueron explicados en términos de la hidrofobicidad y carácter de base débil del herbicida [Hermosin et al., 1992], según un mecanismo que también es válido para explicar la adsorción de IMZ:

$$
\begin{aligned}
& \equiv \mathrm{X}^{-} \mathrm{M}^{+}\left(\mathrm{H}_{2} \mathrm{O}\right)\left(\mathrm{OH}^{-}\right)\left(\mathrm{H}^{+}\right)+\mathrm{IMZ} \\
& \stackrel{(1)}{\leftrightarrow} \equiv \mathrm{X}^{-} \mathrm{M}^{+}\left(\mathrm{H}_{2} \mathrm{O}\right)\left(\mathrm{OH}^{-}\right)\left(\mathrm{H}^{+}\right) \mathrm{IMZ} \\
& \stackrel{(2)}{\leftrightarrow} \equiv \mathrm{X}^{-} \mathrm{IMZH}^{+}+\mathrm{M}\left(\mathrm{H}_{2} \mathrm{O}\right)^{+}+\mathrm{OH}^{-}
\end{aligned}
$$

Figura 3.10 Mecanismo de adsorción de IMZ en Mt.

donde $\equiv \mathrm{X}^{-}$representa los sitios de carga permanente y $\mathrm{M}^{+}$representa un catión inorgánico intercambiable.

En un primer paso, la molécula neutra IMZ es adsorbida en la capa siloxánica hidrofóbica de Mt (Reacción 1, Figura 3.10). En un segundo paso, una molécula de agua de la esfera de hidratación de $\mathrm{M}^{+}$, polarizada, promueve la protonación del anillo imidazol 
(grupo $=\mathrm{N}-\mathrm{H})$ y su interacción electrostática con los sitios de carga permanente de la $\mathrm{Mt}$ (Reacción 2, Figura 3.10). El resultado es el intercambio catiónico entre $\mathrm{M}^{+}$e $\mathrm{IMZH}^{+}$en los sitios de carga permanente con liberación de $\mathrm{OH}^{-}$, lo cual explica el aumento de pH observado en las isotermas de adsorción de IMZ, cuando no se ajusta el pH externamente. Este mecanismo tendría lugar también en las muestras OMt39, OMt55 y OM75 a $C_{f}$ menores a $50 \mathrm{mg} / \mathrm{L}$ y permite explicar el menor valor de $Q$ obtenido para estas muestras respecto al de Mt (Figura 3.9) debido a que en este intervalo de concentraciones la presencia de $\mathrm{ODTMA}^{+}$en los sitios de carga permanente, disminuye la cantidad de cationes $\mathrm{Na}^{+}$y sus respectivas esferas de hidratación (como se identificó en la Sección 3.3.1), disminuyendo a su vez el número de sitios disponibles para la protonación del IMZ en la superficie del sólido. Por el contrario, para la muestra OMt137 este mecanismo no es válido, ya que el contenido de ODTMA supera el $100 \%$ CIC, y por lo tanto en esta muestra no existen cationes $\mathrm{Na}^{+}$en los sitios de carga permanente, inhabilitando la reacción representada en la Figura 3.10. Por lo tanto, otro mecanismo gobierna la adsorción de IMZ en esta muestra, así como también la adsorción de IMZ a $C_{f}$ mayores a $50 \mathrm{mg} / \mathrm{L}$ en las OMts con menor contenido de ODTMA. En el intervalo de $C_{f}$ de 50 a $600 \mathrm{mg} / \mathrm{L}$, la suma de IMZ más ODTMA en las muestras, supera el 100 \%CIC (Figura 3.9), por lo que otros sitios, además de los de carga permanente, deben participar en la interacción con el fungicida.

Si los sitios activos de adsorción de IMZ en las OMts, como se propuso en la hipótesis y en la Sección 2.2 de este trabajo de investigación, fueran las cadenas alquílicas de ODTMA, se esperaría que la adsorción del compuesto se incrementara con el aumento de la fase orgánica. Sin embargo, experimentalmente ocurrió lo contrario (Figura 3.9, Tabla 3.3). En las OMts, el parámetro $Q_{\max }$ aunque aumentó en OMt39 respecto a Mt, luego disminuyó con el aumento de surfactante desde la muestra OMt39 hasta OMt137. Una interpretación de este comportamiento fue propuesto por Jaynes y Boyd [1991], quienes sugirieron que los cationes orgánicos intercalados no estaban directamente involucrados en la adsorción de compuestos orgánicos presentes en agua por parte de las organo-montmorillonitas sino que éstos actuaban como "pilares" manteniendo las láminas separadas para la entrada del adsorbato. Más aún, concluyeron que la capa siloxánica de la montmorillonita (hidrofóbica) era el sitio activo en la adsorción del compuesto. Teniendo en cuenta estas interpretaciones y los resultados aquí obtenidos, se propone que a concentraciones altas de IMZ y de ODTMA, los sitios activos para la unión del fungicida sean la superficie siloxánica, donde las moléculas de IMZ en su forma neutra se unen a través de interacciones van der Waals. 
Las cadenas ODTMA no estarían involucradas directamente en la adsorción del fungicida sino más bien actuarían manteniendo las láminas separadas. Ello explicaría el aumento del valor de $Q$ en las OMts a concentraciones elevadas de IMZ, y su disminución al incrementar el porcentaje de ODTMA. El aumento del contenido de ODTMA genera un mayor recubrimiento de la superficie siloxánica y los sitios activos disponibles para la interacción con el fungicida disminuyen [Jaynes y Boyd, 1991]. Un comportamiento similar reportaron Socias-Viciana et al. [1998] quienes observaron un compromiso entre el espesor de la intercapa y el espacio interlaminar disponible para la sorción del herbicida prometrone en dos organo-montmorillonitas diferentes. De acuerdo con estas observaciones y conforme a los mecanismos descriptos anteriormente, parecería que la presencia de ODTMA es necesaria para cambiar el entorno de la Mt (de hidrofílico a hidrofóbico) y mantener la intercapa abierta pero no actuaría como sitio activo en la adsorción de IMZ.

Resumiendo hasta aquí, se propone que el mecanismo de adsorción de IMZ en Mt (sin ajuste del $\mathrm{pH}$ ) y en OMt39, OMt55 y OMt75 (a concentraciones bajas de IMZ), sea el intercambio catiónico entre $\mathrm{IMZH}^{+}$y $\mathrm{Na}^{+}$en los sitios de carga permanente según el esquema descripto en la Figura 3.10. Para las OMts se propone que, a cantidades adsorbidas que superan el $100 \%$ CIC, y especialmente para la muestra OMt137, tenga lugar otro mecanismo que se suma al anterior, esto es, la penetración de IMZ en forma neutra en las galerías hidrofóbicas formadas por las aminas intercaladas en la intercapa.

En línea con este razonamiento, se esperaría que la adsorción de IMZ en Mt ocurriera hasta alcanzar el $100 \%$ CIC. La ausencia de este comportamiento cuando el pH de la isoterma no es regulado externamente $\left(Q_{\max }=155 \mathrm{mg} / \mathrm{g}\right.$ corresponde $\left.63 \% \mathrm{CIC}\right)$ no puede comprenderse si no se tiene en cuenta que, además de la reacción ácido-base del IMZ (Figura 1.2), existen otros equilibrios ácido-base en el sistema (reacciones que pueden experimentar los sitios de bordes de Mt, Sección 2.1.2). Indudablemente, en las condiciones de trabajo el sistema deja de evolucionar hacia productos sin que los sitios se saturen cuando todos los procesos alcanzan el equilibrio.

Sin embargo, cuando el $\mathrm{pH}$ se mantiene en un valor neutral a lo largo de todo el intervalo de concentraciones de IMZ (Figura 3.9, Tabla 3.3), se observó un aumento de $Q_{\max }$ a $363 \mathrm{mg} / \mathrm{g}$ en $\mathrm{Mt}$, el cual es 2,34 veces más grande que el obtenido por la misma muestra sin ajuste de $\mathrm{pH}$ y corresponde al $148 \% \mathrm{CIC}$. El hecho de que el porcentaje adsorbido exceda el 100 \% CIC indica que, aparte del intercambio catiónico en los sitios de carga permanente, otro mecanismo y otros sitios activos de la Mt deben estar involucrados en la adsorción del fungicida. 
Para dilucidar este efecto, se realizaron curvas de porcentaje de remoción $(E)$ en función del pH (Figura 3.11), donde se observa (y se confirma) que la remoción de IMZ por parte de la Mt es fuertemente dependiente del $\mathrm{pH}$. Teniendo en cuenta que los sitios de carga permanente no dependen del $\mathrm{pH}$, esto indica que los sitios de borde participan en la adsorción del fungicida y explican que la cantidad adsorbida supere el $100 \%$ CIC.

La eficiencia de la remoción disminuye cuando el $\mathrm{pH}$ es menor a 4. En esas condiciones todo el fungicida se encuentra como $\mathrm{IMZH}^{+}$, y los sitios de borde se encuentran mayoritariamente con carga positiva [Pecini y Avena, 2013], por lo tanto la diminución de $E$ al disminuir el $\mathrm{pH}$ por debajo del $\mathrm{pH}_{\mathrm{PZC}, \text { borde }}$ se puede comprender por la repulsión electrostática entre los sitios y el adsorbato. A valores de $\mathrm{pH}$ superiores a $\mathrm{pH}_{\mathrm{PZC}, \text { borde }}$ e inferiores a 7,5, los sitios de borde se encuentran mayoritariamente con carga negativa; hasta $\mathrm{pH}=6,5$ la especie $\mathrm{IMZH}^{+}$es mayoritaria respecto a IMZ, con lo cual la adsorción en estos sitios está favorecida. Además, la concentración de $\mathrm{H}^{+}$es suficiente para desplazar el equilibrio de la Figura 3.10 hacia la derecha, por el principio de Le Chatellier, por lo que la adsorción en los sitios de carga permanente también es posible. A valores de $\mathrm{pH}$ básicos y superiores a 7,5, E vuelve a disminuir; la concentración de $\mathrm{OH}^{-}$aumenta desplazando el equilibrio en la Figura 3.10 hacia la izquierda. El IMZ está mayoritariamente en su forma neutra y los bordes con carga negativa, con lo cual la adsorción en estos sitios tampoco se ve favorecida.

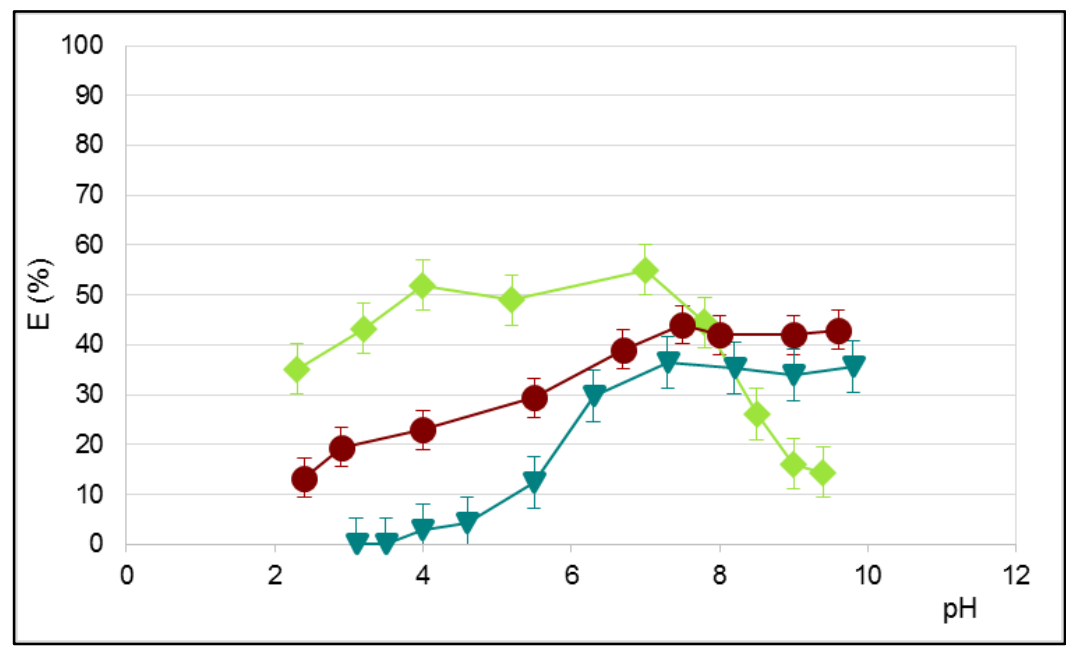

Figura 3.11 Eficiencia de remoción de IMZ $\left(C_{i}=600 \mathrm{mg} / \mathrm{L}\right)$ vs. pH de $(\bullet) \mathrm{Mt},(\bullet)$ OMt39 y $(\boldsymbol{\nabla})$ OMt137.

Las curvas de $E$ vs. $\mathrm{pH}$ para las muestras con menor y mayor contenido de ODTMA (OMt39 y OMt137) evidenciaron que la adsorción está favorecida a valores de $\mathrm{pH}$ neutros y básicos, a diferencia de la Mt natural que mostró que su pH óptimo de adsorción fue el 
neutro y/o ligeramente ácido. Este comportamiento es coherente con el mecanismo propuesto: a $\mathrm{pH}$ básicos, la forma neutra del fungicida se adsorbe principalmente en la superficie hidrofóbica de las OMts. Además, la caída de $E$ a $\mathrm{pH}$ menor a $\mathrm{p} K_{a}(\mathrm{IMZ})=6,5$ probablemente se deba a la repulsión electrostática entre $\mathrm{IMZH}^{+}$y $\mathrm{ODTMA}^{+}$que podría disminuir la capacidad adsorbente de las organo-montmorillonitas. Este efecto sería más pronunciado en la muestra OMt137 que en la OMt39, ya que la primera presenta carga externa positiva en todo el intervalo de $\mathrm{pH}$.

\subsubsection{Caracterización de productos de adsorción}

Los difractogramas de rayos $\mathrm{X}$ de los productos de adsorción con IMZ (Tabla 3.4) evidenciaron un incremento en el espaciado basal comparados con las muestras iniciales, señalando la importancia de los sitios interlaminares (sitios de carga permanente y superficie siloxánica) en la adsorción del mismo. El espaciado basal de Mt aumentó 0,66 $\mathrm{nm}$; mientras que las muestras OMts evidenciaron aumentos desde 0,32 nm (IMZ-OMt39) hasta 1,48 nm (IMZ-OMt137) (Tabla 3.4, Figura 3.2), indicando el ingreso del IMZ en el espaciado interlaminar. Los distintos valores de $\mathrm{d}_{001}$ se podrían atribuir a distintos contenidos y/o configuraciones de IMZ en la zona interlaminar.

Tabla 3.4 Espaciado basal, temperatura de descomposición del contenido orgánico (DTg) y potencial zeta $(\mathrm{pH}=7)$ de IMZ y productos de adsorción $\left(C_{i}=600 \mathrm{mg} / \mathrm{L}\right)$.

\begin{tabular}{|c|c|c|c|c|}
\hline Muestra & $\begin{array}{c}\text { Espaciado } \\
\text { basal }[\mathrm{nm}]\end{array}$ & $\begin{array}{c}\text { Potencial zeta } \\
{[\mathrm{mV}]}\end{array}$ & $\begin{array}{c}\text { Descomposición } \\
\text { de ODTMA }\left[{ }^{\circ} \mathrm{C}\right]\end{array}$ & $\begin{array}{c}\text { Descomposición } \\
\text { de IMZ }\left[{ }^{\circ} \mathrm{C}\right]\end{array}$ \\
\hline IMZ & n.c. & n.c. & n.c. & 285 \\
IMZ-Mt & 1,82 & $-18,4 \pm 0,61$ & n.c. & 289 \\
IMZ-OMt39 & 1,75 & $-17,49 \pm 0,57$ & 265 & 306 \\
IMZ-OMt55 & 3,22 & $-28,09 \pm 0,72$ & 263 & 311 \\
IMZ-OMt75 & 3,13 & $-19,20 \pm 0,83$ & 262 & 311 \\
IMZ-OMt137 & 3,50 & $19,07 \pm 0,98$ & 259 & 311 \\
\hline
\end{tabular}

n.c.: no corresponde

Las medidas de potencial zeta indicaron cambios en la carga externa de los adsorbentes luego de la adsorción de IMZ, lo cual permitió inferir que los sitios de superficie externa también estuvieron involucrados en la adsorción del fungicida. Para Mt, se observó una disminución de la carga negativa (Tabla 3.4, Figura 3.3). La muestra IMZ-OMt137, mostró 
por el contrario, una disminución en la carga positiva desde +40 a $+19,7 \mathrm{mV}$ (Tabla 3.4, Figura 3.3). Probablemente, el contenido de ODTMA superior al valor de la CIC, permite la formación de micelas mixtas IMZ-ODTMA [Zhang et al., 2012]. Esto provocaría un cambio en el arreglo de los cationes en la superficie externa (a mayores distancias de la lámina siloxánica) y explicaría también la menor capacidad de adsorción de esta muestra respecto de las restantes OMts. Para las muestras OMt39, OMt55 y OMt75, la adsorción de IMZ produjo disminuciones menores de la carga negativa respecto de la muestra Mt, que también podrían estar asignadas a la formación de micelas mixtas IMZ-ODTMA en la superficie externa.

Los productos de adsorción presentaron en DTg un pico nuevo y bien diferenciado (Figura 3.12) correspondiente a la oxidación de IMZ. Este pico se ubicó a $289^{\circ} \mathrm{C}$ en IMZ-Mt, y alrededor de $311^{\circ} \mathrm{C}$ en las muestras IMZ-OMts (Tabla 3.4), es decir, en todos los casos a temperaturas más elevadas que la del fungicida puro $\left(285^{\circ} \mathrm{C}\right.$, Figura 3.12). Esto evidenció la interacción entre el IMZ y las capas siloxánicas de Mt. Al mismo tiempo, la adsorción de IMZ en las OMts, disminuyó la energía de interacción entre ODTMA y la superficie de la montmorillonita, corriendo el pico DTg hacia $262{ }^{\circ} \mathrm{C}$ aproximadamente para todas las muestras. Como indicaron Zhang et al. [2012], la co-adsorción de un surfactante catiónico y una molécula neutra en un mineral de arcilla puede llevar a una reducción de la estabilidad térmica de las organo-montmorillonitas, y esta reducción es mayor cuanto mayor es el contenido de surfactante.
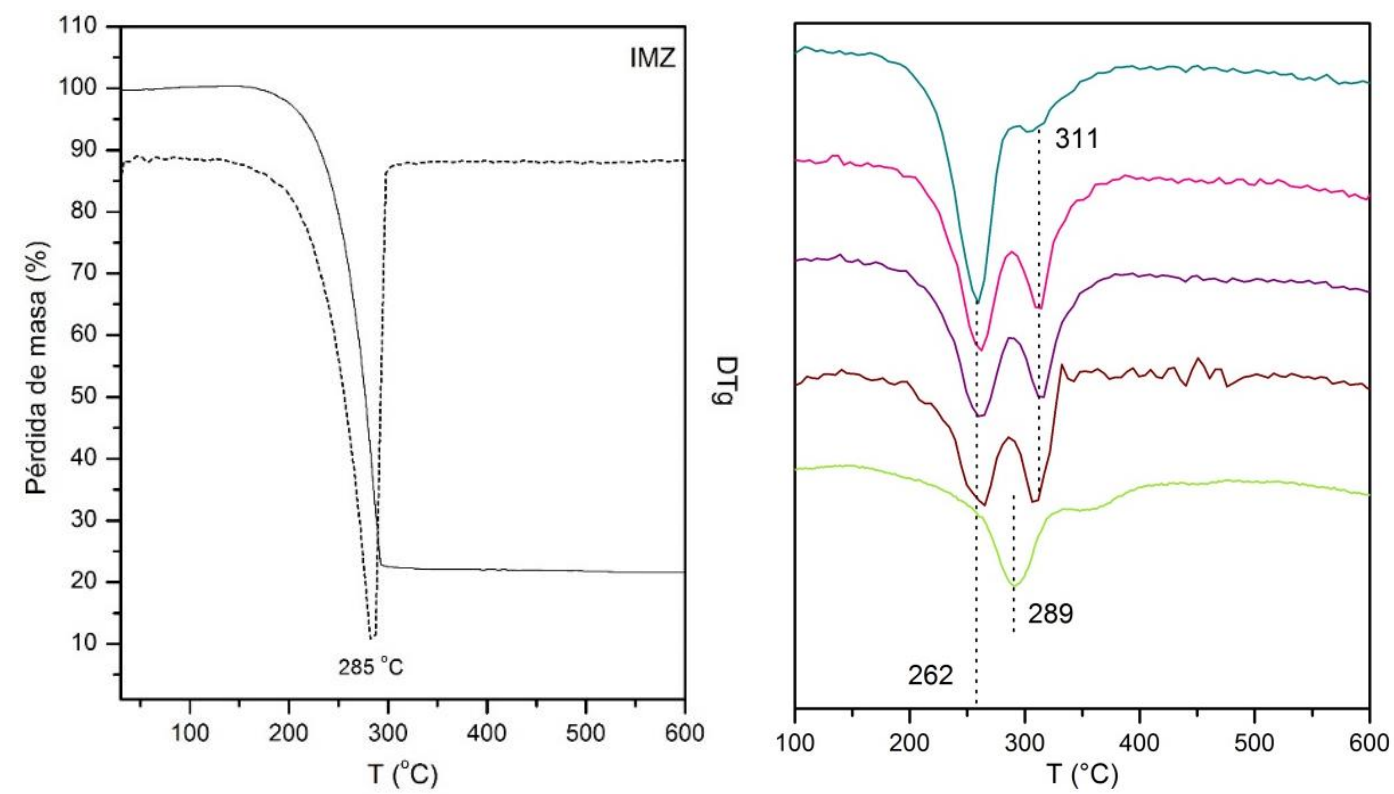

Figura 3.12 Curvas de análisis térmicos. Izquierda: Tg y DTg de IMZ. Derecha: DTg de $(-)$ IMZ-Mt, $(-)$ IMZ-OMt39, (-) IMZ-OMt55, (-) IMZ-OMt75 y (-) IMZ-OMt137. 
Los espectros MIR y NIR de los productos de adsorción permitieron dilucidar los cambios estructurales que provocó la adsorción del fungicida y confirmó el mecanismo propuesto anteriormente. El espectro MIR del IMZ puro se puede dividir en tres regiones espectrales (Figura 3.13). En la primera, entre 3600-2600 $\mathrm{cm}^{-1}$, ocurren las transiciones vibracionales del estiramiento $\mathrm{C}-\mathrm{H}$ alifáticos, vinílicos y aromáticos. En la segunda, entre 1800-1380 $\mathrm{cm}^{-1}$ ocurren las transiciones vibracionales del estiramiento C-C vinílicos y aromáticos. En la última entre 1380-500 $\mathrm{cm}^{-1}$, aparecen las vibraciones de deformación de los grupos C-H (Tabla A1). En el espectro NIR (Figura 3.14), las bandas en la región entre 6200-5700 $\mathrm{cm}^{-1}$ corresponden al primer sobretono del estiramiento C-H de los grupos alifáticos, vinílicos y aromáticos, mientras que la combinación de modos vibracionales de estiramiento C-H y C-C aromáticos ocurren en la zona $4600-4400 \mathrm{~cm}^{-1}$. Las bandas por debajo de $4400 \mathrm{~cm}^{-1}$ se asignaron a la combinación de modos de vibraciones de los enlaces C-H (Tabla A2).

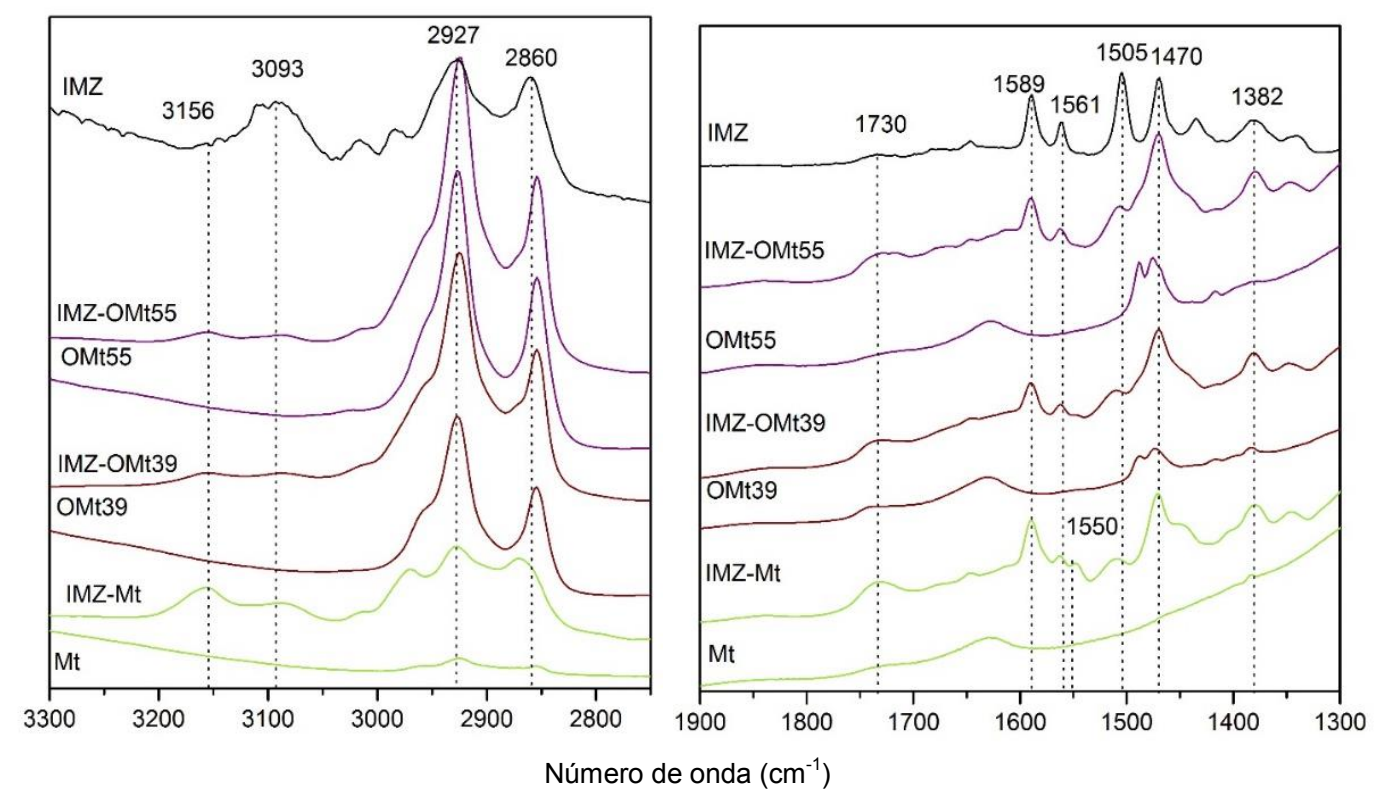

Figura 3.13 Espectros MIR de IMZ y productos de adsorción indicados.

Luego de la adsorción de fungicida en Mt, el espectro del material se vio modificado por la aparición de bandas características del fungicida (Figura 3.13). Los modos vibracionales a 3089; 2928; 2871; 1590; 1471 y $1380 \mathrm{~cm}^{-1}$ en IMZ-Mt se asignaron al IMZ (Tabla A1). La protonación del grupo imidazol que se propuso para explicar la adsorción del fungicida en la muestra Mt, se confirmó por la aparición de nuevas bandas situadas a 3155 y $1550 \mathrm{~cm}^{-1}$ en el espectro IMZ-Mt (Figura 3.13). Estas bandas, características de la protonación de piridinas, evidencian los sitios ácidos en minerales de arcilla [Yariv, 2001], y corresponden a 
los modos vibracionales de estiramiento y deformación del grupo $\mathrm{NH}^{+}$, respectivamente. Una disminución significativa de la intensidad de los picos $v \mathrm{NH}^{+}$y $\delta \mathrm{NH}^{+}$en los espectros MIR de las OMts podría generarse por la disminución del contenido de agua de las organomontmorillonitas respecto al de la $\mathrm{Mt}$, en concordancia con los datos de las isotermas de adsorción y el mecanismo propuesto para explicarlo. Más aún, la banda intensa $\mathrm{C}=\mathrm{C}$ aromático (Ar) cerca de $1560 \mathrm{~cm}^{-1}$ posiblemente se superpone con el pico correspondiente a $\delta \mathrm{NH}^{+}$. La banda a $6200 \mathrm{~cm}^{-1}$ en el espectro NIR, mejor definida en IMZ-Mt que en IMZ-OMts (Figura 3.14), se asignó al primer sobretono de $v \mathrm{NH}^{+}$y también confirmó la protonación del anillo imidazol en las muestras.

A pesar de que la asignación de las bandas MIR de IMZ en las organo-montmorillonitas no es trivial debido a la superposición con las vibraciones del ODTMA, los picos alrededor de 3093; 1589; 1561; $1505 \mathrm{~cm}^{-1}$ (propias del fungicida puro, Tabla A1) confirmaron la adsorción del IMZ en las OMts (Figura 3.13). No se observaron diferencias entre las formas ni las posiciones de las bandas del ODTMA antes y después de la adsorción, confirmando que el surfactante no estuvo involucrado directamente en la interacción con el fungicida. Por el contrario, se observaron, además de la aparición de las bandas correspondientes a la protonación del $\mathrm{N}$, cambios en la forma de la banda a $7080 \mathrm{~cm}^{-1}$ para todos los adsorbentes. El IMZ no presenta bandas en esta zona; sin embargo, se observó la aparición de un hombro en la banda a $7170 \mathrm{~cm}^{-1}$, correspondiente a $2 \vee \mathrm{OH}$, en todos los productos de adsorción, que se asignó al cambio en el entorno químico de los grupos estructurales $\mathrm{OH}$ de los adsorbentes [Madejová et al., 2011]. Estos resultados confirmaron que los sitios activos de adsorción del fungicida fueron los sitios de carga permanente y la superficie siloxánica, mientras que el ODTMA actuó manteniendo las láminas separadas y permitiendo la interacción entre el fungicida y las hojas tetraédricas. La posición e intensidad relativa de la banda $(\nu+\delta) \mathrm{H}_{2} \mathrm{O}\left(5246 \mathrm{~cm}^{-1}\right)$ también se modificó luego de la adsorción del IMZ (Figura 3.14, Tabla A2). En todos los casos esta banda se corrió hacia mayores números de onda indicando uniones hidrógeno de carácter más débil entre las moléculas de agua y la superficie de la Mt. Más aún, la intensidad de esta banda disminuyó significativamente luego de la adsorción del fungicida, indicando el aumento de la hidrofobicidad de los productos de adsorción respecto de los adsorbentes [Madejová et al., 2011]. 

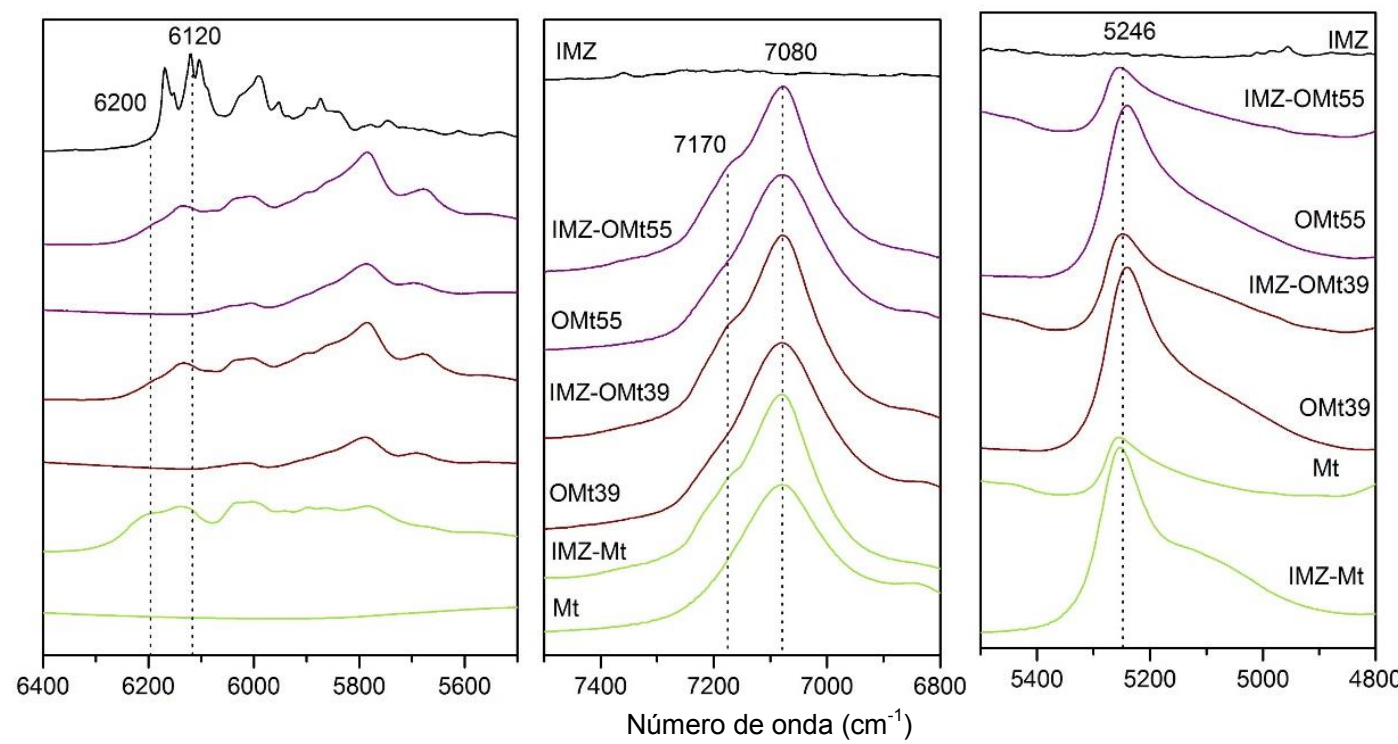

Figura 3.14 Espectros NIR de IMZ y productos de adsorción indicados.

La adsorción de IMZ en la superficie de la Mt también modificó la posición del primer sobretono de C-H del grupo vinilo. Mientras que para el IMZ esta banda se observó a 6120 $\mathrm{cm}^{-1}$, en el espectro NIR de las muestras IMZ-Mt e IMZ-OMts se observó un corrimiento a $6130 \mathrm{~cm}^{-1}$ (Figura 3.14), probablemente como resultado de la interacción entre el grupo vinilo y los adsorbentes.

Por último, la aparición de una nueva banda a $\sim 1730 \mathrm{~cm}^{-1}$ en el espectro de todos los productos de adsorción (Figura 3.13) indicaría la existencia de otro proceso. Como el único grupo funcional que absorbe radiación a esa frecuencia es el grupo carbonilo, es probable que algunas moléculas de IMZ hayan sufrido una transformación a su derivado (imazalil etanol, [Yoshioka et al., 2004]) y una posterior oxidación del grupo $\mathrm{OH}$ a carbonilo.

\subsection{Conclusiones parciales}

En este capítulo se obtuvieron y caracterizaron diferentes organo-montmorillonitas empleando como catión de intercambio una amina cuaternaria, con una cadena alquílica de 18 carbonos. A través de las técnicas utilizadas, pudo establecerse que la amina se unió a los sitios de carga permanente cuando su porcentaje fue menor al $100 \% \mathrm{CIC}$, mientras que sumados a estos sitios también participaron los de superficie externa, cuando el porcentaje de intercambio superó dicho valor. El arreglo del surfactante en la intercapa así como el tamaño de los agregados dependió del contenido de ODTMA. El tamaño de partícula medido como diámetro aparente creció un orden de magnitud en las organo- 
montmorillonitas respecto a la montmorillonita, lo cual supone una mayor facilidad de separación del sólido de la suspensión en las OMts respecto a la Mt.

Se evaluó la capacidad de adsorción de IMZ de las organo-montmorillonitas así como de la montmorillonita. Los materiales presentaron un desempeño comparable y mejor que los carbones activados sintetizados por Martin-González et al. [2014].

A partir de las isotermas de adsorción, de curvas de eficiencia de remoción vs. $\mathrm{pH}, \mathrm{y}$ de la caracterización de los productos de adsorción, pudo determinarse que el mecanismo de adsorción, así como los sitios activos involucrados, dependen de las condiciones tanto de $\mathrm{pH}$, como de contenido de ODTMA en las muestras y de la concentración de IMZ.

En Mt, los sitios activos de unión del IMZ fueron principalmente los de carga permanente, aunque los de superficie externa y de borde también estuvieron involucrados. En particular, estos últimos explican la adsorción del fungicida a porcentajes mayores al $100 \% \mathrm{CIC}$, cuando se realiza la isoterma ajustando el pH a 7. El mecanismo de adsorción identificado consiste en la protonación del fungicida en la superficie del material, y su unión electrostática con la lámina siloxánica, de carga negativa. Este mecanismo corresponde a un intercambio catiónico, donde como productos se liberan $\mathrm{Na}^{+}$y $\mathrm{OH}^{-}$al medio acuoso, explicando el incremento de $\mathrm{pH}$ encontrado durante la adsorción.

Respecto a la adsorción de IMZ en organo-montmorillonitas, se identificó que cuando la suma de cantidad adsorbida de IMZ y ODTMA es menor al $100 \%$ CIC, los sitios de adsorción son, principalmente, los sitios de carga permanente, donde el $\mathrm{IMZH}^{+}$se intercambia por el $\mathrm{Na}^{+}$remanente en el sólido. Cuando el contenido de IMZ y/o de ODTMA supera el 100 \%CIC, otro mecanismo tiene lugar. En este caso intervienen, además de los sitios de carga permanente, la superficie siloxánica que alberga a la forma neutra del fungicida, mediante interacciones de van der Waals. Porcentajes bajos de ODTMA actúan manteniendo las capas separadas y favorecen la entrada del fungicida en la zona interlaminar, mientras que cantidades elevadas de ODTMA recubren la superficie siloxánica, y por lo tanto actúan disminuyendo el número de sitios disponibles para la unión de IMZ. Este mecanismo explica que la cantidad adsorbida por unidad de masa disminuya con el aumento de ODTMA. Se pudo evidenciar que las cadenas alquílicas no estuvieron involucradas como sitios activos. Es decir que la hipótesis planteada acerca de que la modificación de la Mt con cadenas alquílicas generaría un incremento en la remoción de compuestos orgánicos por el mecanismo de partición organofílica, no se cumplió para el IMZ. 


\section{Capitulo 4. \\ Adsorción de tiabendazol en montmorillonitas y organo-montmorillonitas.}

\subsection{Introducción y objetivos específicos}

La adsorción de tiabendazol (TBZ) en montmorillonitas ha sido reportada en diversas publicaciones desde el año 1975, donde Aharonson y Kafkafi [1975b] estudiaron la remoción de fungicidas derivados del bencimidazol en una montmorillonita de referencia internacional (Wyoming) homoionizada con $\mathrm{Na}^{+} \mathrm{y} \mathrm{Ca}^{2+}$ y sobre una caolinita. Estos autores estudiaron la dependencia de la adsorción con el pH y con la fuerza iónica. El aumento en la remoción del TBZ en dichos materiales, al disminuir el $\mathrm{pH}$ de 5 a 2, se relacionó con la interacción de la superficie de la montmorillonita con la especie protonada del compuesto $\left(\mathrm{TBZH}^{+}\right.$, Figura 1.2). Este resultado lo explicaban aduciendo que, en presencia de mayor concentración de iones protones, el TBZ estaría mayoritariamente con carga positiva favoreciendo la interacción electrostática con el sólido. Entre pH 5 y 7,8 la curva de adsorción vs. pH experimentaba un aumento, alcanzando la máxima cantidad adsorbida

\footnotetext{
${ }^{6}$ Se agradece especialmente a Petr Kovář y Miroslav Pospíšil, de la Facultad de Matemática y Física de la Charles University en Praga (República Checa) por recibirme hospitalariamente en su oficina, y por su colaboración en este capítulo en lo que respecta a simulaciones moleculares; y a José Luis "Pupi" Borioni, amigo querido, del Instituto de Investigaciones en Físico-Química de Córdoba (Universidad Nacional de Córdoba-CONICET) por la lectura final del capítulo y sus valiosos aportes.
} 
por unidad de masa a $\mathrm{pH}=7,8(21,5 \mathrm{mg} / \mathrm{g})$ y disminuyendo hacia valores de $\mathrm{pH}$ más básicos.

Años más tarde, Draoui et al. [1998] realizaron un estudio termodinámico de la adsorción de pesticidas disueltos en etanol en componentes de suelos (entre ellos la montmorillonita de Wyoming), mostrando que el comportamiento de las isotermas eran principalmente del tipo Langmuir y sugiriendo que el TBZ se intercalaba entre las láminas de la montmorillonita.

Un trabajo más completo para comprender los sitios y mecanismos de unión de TBZ en montmorillonita fue realizado por Lombardi et al. [2003; 2006]. En dichos trabajos se estudió la influencia de los parámetros: tiempo, $\mathrm{pH}$, fuerza iónica, relación sólido/solución y se caracterizó el producto de adsorción de TBZ en la montmorillonita provista por Castiglioni Pes y Cia (Mt). Los parámetros de trabajo que resultaron óptimos, tanto para maximizar el desempeño de los adsorbentes como para que el procedimiento fuera operativamente sencillo en la aplicación industrial, fueron: tiempo de contacto $=24 \mathrm{~h}$; relación sólido/solución $=1 \mathrm{~g} / \mathrm{L} ; \mathrm{pH}$ y fuerza iónica sin ajuste. La equivalencia entre los cationes $\mathrm{Na}^{+}$removidos y las moléculas de TBZ adsorbidas; la aparición en el espectro infrarrojo del producto de adsorción de una señal asociada al grupo N-H del bencimidazol (protonado); la comparación de las isotermas de adsorción de TBZ con las de colorantes catiónicos; y la ausencia de desorción aún luego de 30 días fueron evidencias concluyentes que llevaron a los autores a explicar la adsorción a través de la siguiente ecuación química:

$$
\equiv \mathrm{X}^{-} \mathrm{Na}^{+}+\mathrm{TBZ}+\mathrm{H}_{2} \mathrm{O} \rightarrow \equiv \mathrm{X}^{-}\left(\mathrm{TBZH}^{+}\right)+\mathrm{Na}^{+}+\mathrm{OH}^{-}
$$

donde $\equiv \mathrm{X}^{-}$representa los sitios de carga permanente. Los autores sugirieron un intercambio catiónico entre el $\mathrm{TBZH}^{+}$y el $\mathrm{Na}^{+}$presente inicialmente en la intercapa y propusieron como mecanismo la co-adsorción de TBZ y $\mathrm{H}^{+}$en los sitios de carga permanente, lo cual explicaba el aumento de $\mathrm{pH}$ observado durante los experimentos de adsorción. Los estudios de DRX, potencial zeta y XPS además, permitieron identificar los sitios activos, siendo éstos los de carga permanente (donde el TBZ se disponía en forma plana, paralela a las láminas T:O:T) y los de superficie externa. Se hace notar que en el trabajo de Lombardi et al. [2003] se alcanzaron cantidades adsorbidas superiores a 1000 $\mathrm{mg} / \mathrm{g}$, pero allí se utilizaron soluciones concentradas de TBZ con un porcentaje importante de etanol.

En 2013, Roca Jalil et al., reportaron la adsorción de TBZ comercial, disuelto en soluciones acuosas, en otra montmorillonita proveniente del Alto Valle de Río Negro y la 
compararon con montmorillonitas modificadas por pilareado con hidróxidos de aluminio. En líneas generales, estos autores observaron que las montmorillonitas modificadas presentaron capacidades de adsorción considerablemente mayores a la de la arcilla natural en todos los casos (expresado en el parámetro de Langmuir, $Q_{\max }$ de 11,91 mg/g a 63,87 $\mathrm{mg} / \mathrm{g}$ ) y sugirieron que ello se debía a la existencia de un mecanismo de adsorción diferente al de intercambio catiónico. Otra ventaja que resaltaron de los materiales pilareados fue su hidrofobicidad, propiedad que evitó la formación de suspensiones estables y en consecuencia, promovió la sedimentación al eliminar la agitación del sistema (a diferencia de la montmorillonita natural que forma suspensiones estables inclusive luego de $24 \mathrm{~h}$, Figura A1). Este hecho, les permitió a los autores proponer estos adsorbentes para su aplicación en los tratamientos de efluentes reales, con el consecuente costo de preparación de los mismos.

Teniendo en cuenta estos antecedentes, en este capítulo se buscó comparar el desempeño de dos montmorillonitas, una nacional y una internacional, y organomontmorillonitas obtenidas a partir de las primeras. Se plantearon como objetivos específicos:

(i) evaluar la adsorción de TBZ en dos montmorillonitas (una nacional y una de referencia a nivel internacional) y organo-montmorillonitas obtenidas a partir de éstas (con bromuro de octadeciltrimetilamonio en dos porcentajes distintos respecto de la CIC);

(ii) caracterizar productos de adsorción de tiabendazol, dilucidar sitios activos involucrados y proponer un mecanismo de adsorción del fungicida en los adsorbentes estudiados.

\subsection{Materiales y métodos}

\subsubsection{Materiales}

El adsorbato utilizado fue tiabendazol en grado estándar provisto por Sigma-Aldrich. Se preparó una solución acuosa de concentración $=25 \mathrm{mg} / \mathrm{L}$ y todas las soluciones se prepararon en agua destilada a partir de esta solución madre.

Como adsorbentes se evaluaron las muestras Mt, OMt55 y OMt137 cuyas caracterizaciones se presentaron en el Capítulo 3. La capacidad de adsorción de éstas se compararon con las de una montmorillonita de referencia internacional, Wyoming $\mathrm{Na}$ montmorillonite (Wy), (suministrada por Source Clays Repository of The Clay Minerals 
Society (Columbia, MO) y utilizada sin tratamiento previo) y con las de una organomontmorillonita obtenida a partir de ésta y bromuro de octadeciltrimetilamonio (OWy).

La Wy fue caracterizada previamente en [Marco-Brown, 2011] donde se determinó su punto isoeléctrico $=3,2$; superficie específica $=27 \mathrm{~m}^{2} / \mathrm{g}$; superficie específica total $=632$ $\mathrm{m}^{2} / \mathrm{g}$ y fórmula estructural $=\left[\mathrm{Si}_{3,94} \mathrm{Al}_{0,06}\right]\left(\mathrm{Al}_{1,56} \mathrm{Fe}^{3+}{ }_{0,18} \mathrm{Mg}_{0,26}\right) \mathrm{O}_{10}(\mathrm{OH})_{2} \mathrm{M}_{0,32}^{+}$. La mineralogía indicó montmorillonita sódica como fase predominante (98\%) con cuarzo y feldespato como fases minoritarias. Su capacidad de intercambio catiónico (CIC) es $0,92 \mathrm{mmol} / \mathrm{g}$ determinada por el método de azul de metileno [Tabak et al., 2006].

La OWy se obtuvo llevando a cabo el procedimiento que se describió en la Sección 3.2.1. Se empleó una cantidad de ODTMA equivalente a $211 \% \mathrm{CIC}$ de Wy, para obtener una muestra con contenido similar de surfactante al presente en OMt137.

\subsubsection{Métodos de caracterización}

En este capítulo se presentan las caracterizaciones de Wy y OWy y productos de adsorción con TBZ (que se denominaron anteponiendo TBZ- al nombre del adsorbente) mediante DRX, potencial zeta y análisis térmicos en las condiciones indicadas en la Sección 3.2.2. Mediante análisis térmicos se realizó una estimación del contenido de amina en la muestra OWy. Además, las muestras Wy, TBZ-Wy, OWy y TBZ-OWy fueron caracterizadas mediante espectroscopía de fotoelectrones (XPS) y simulaciones computacionales.

\subsubsection{Espectroscopía de fotoelectrones (XPS)}

La espectroscopia de fotoelectrones (XPS) determina la energía de unión de los electrones más cercanos al núcleo de un átomo, mediante su excitación por rayos $\mathrm{X}$ y posterior evaluación de su decaimiento, lo que permite caracterizar el estado de oxidación de un átomo presente en un sólido.

La técnica XPS utiliza como fuentes de excitación las líneas $\mathrm{K} \alpha_{1,2}$ de $\mathrm{Mg}$ o $\mathrm{Al}$ que tienen energías de 1253,6 y $1486 \mathrm{eV}$, respectivamente. La energía cinética de los fotoelectrones, una vez que salen de la superficie de la muestra, se mide por varias técnicas siendo la más común la de cambio de dirección de los electrones mediante analizadores electrostáticos o magnéticos. Estos analizadores electrostáticos permiten medir la energía cinética que el equipo transforma en energía de enlace.

Como solamente los electrones que han interaccionando en la superficie de la muestra pueden alcanzar el detector para ser analizados, la técnica XPS es superficial (informa sobre tres o cuatro capas de átomos de la superficie de la muestra). Es por ello que en este trabajo 
se utilizó esta técnica con el objetivo de caracterizar la superficie externa de las muestras Wy, OWy y sus productos de adsorción, tanto cualitativamente -mediante la identificación de los elementos presentes antes y después de la adsorción (en concentraciones mayores al 0,1\%)- como también mediante el análisis semi-cuantitativo de la composición elemental de la superficie (error $< \pm 10 \%)$.

Los análisis XPS se realizaron en un espectrómetro Multithecnich Specs con Rayos-X duales con fuente de $\mathrm{Mg} / \mathrm{Al}$ y XR50, modelo Phoibos 150, analizador hemiesférico, con analizador modo de transmisión fija (FAT). Se obtuvieron los espectros de alta resolución de la señal $1 s$ de $\mathrm{Na}, \mathrm{O}, \mathrm{N}$ y $\mathrm{C} ; 2 p$ de $\mathrm{Fe}, \mathrm{Br}, \mathrm{Si}$ y Al y $2 s$ del Mg. Los espectros fueron obtenidos con un paso de energía de $30 \mathrm{eV}$ y ánodo de $\mathrm{Al}$ operando a $100 \mathrm{~W}$. Las muestras fueron soportadas en una cinta de cobre y sometidas a ultra-alto vacío $\left(2.10^{-6} \mathrm{~Pa}\right)$ durante $12 \mathrm{~h}$ previas al análisis. Para minimizar los efectos de carga superficial, se trabajó con energía de ignición Flood Gun 1eV. La componente C- $(\mathrm{C}, \mathrm{H})$ del pico C1s se fijó a $285 \mathrm{eV}$ para ajustar la escala de energía de enlace. Las fracciones molares se calcularon usando las áreas de los picos normalizadas en base a los parámetros de adquisición, luego de substraer el background. Factores de sensibilidad, camino libre medio y factores de transmisión fueron provistos por el fabricante.

\subsubsection{Simulaciones computacionales}

El modelado molecular es un conjunto de técnicas basadas en aplicaciones informáticas que permiten la construcción de modelos moleculares tridimensionales, análisis de las posibles conformaciones con sus energías y obtención de propiedades mediante Mecánica Molecular (MM) y/o Métodos Cuánticos (MC).

En particular, la MM es una herramienta valiosa que emplea las leyes de la física clásica para obtener propiedades de los sistemas moleculares de interés. Además, representa una alternativa a los MC debido a que resolver la ecuación de Schrödinger para sistemas grandes -de varios cientos de átomos- implica un costo computacional considerable.

En los cálculos de MM, los átomos del sistema son representados como esferas rígidas cargadas interactuantes en las cuales los electrones no son considerados explícitamente. La ecuación matemática que describe las tensiones a las cuales están sometidos dichos átomos, más el conjunto de tipo de átomos y parámetros que ajustan las ecuaciones a la información experimental, se denomina campo de fuerza.

La expresión matemática del campo de fuerza define la energía potencial del sistema, la cual varía con las posiciones de los átomos. Esta expresión se construye como la sumatoria de términos asociados a energías de interacción entre pares de átomos enlazados y no 
enlazados. Los términos enlazantes incluyen contribuciones debidas a los enlaces covalentes, ángulos de enlace y ángulos de torsión. Los términos no enlazantes se definen por un término de atracción-repulsión del tipo Lennard-Jones para las fuerzas de van der Waals y un término coulómbico para las interacciones electrostáticas. El conjunto de tipo de átomos en un campo de fuerza define diferentes características y desempeño de un elemento dependiendo de su entorno químico. Es decir, un mismo elemento es tratado de manera diferente según su hibridización, carga y a qué átomos se encuentra unido. Por último, los parámetros que ajustan las ecuaciones y tipos de átomos a la información experimental, se encuentran definidos por las constantes de fuerza, parámetros de van der Waals y cargas atómicas. Estos parámetros son empleados en las ecuaciones para relacionar características atómicas y estructurales con las componentes de la energía potencial.

Entre las técnicas basadas en la MM, la Dinámica Molecular (DM) permite generar configuraciones sucesivas de un sistema integrando las leyes del movimiento de Newton. Las ecuaciones de Newton del movimiento para un sistema de $N$ partículas interactuando a través de un potencial $U\left(r_{i}\right)$, con $i=1, \ldots, N$ pueden ser formuladas en ecuaciones diferenciales de segundo orden del tipo

$$
F_{i}=m_{i} \frac{\partial^{2} r_{i}}{\partial t^{2}}
$$

donde $r_{i}$ representa las coordenadas de la partícula y $F_{i}$ la fuerza. Ésta, depende de la energía potencial según

$$
F_{i}=-\frac{\partial U\left(r_{i}\right)}{\partial r_{i}}
$$

Estas ecuaciones deben ser integradas usando algún método de diferenciación finita a partir de potenciales continuos, que sólo involucran interacción de a pares. La integración de las ecuaciones de diferenciación finita se realiza en diferenciales de tiempo, $\delta t$. Éstos, se denominan pasos de integración y presentan un valor fijo a lo largo de todo el tiempo de simulación.

La fuerza total sobre un átomo en un tiempo tes calculada como la sumatoria de todas las fuerzas que actúan sobre éste (Ecuación 4.3). A partir de la fuerza, se puede conocer la aceleración de las partículas, la cual es combinada con las posiciones y velocidades en el tiempo $t$ para calcular las posiciones y velocidades en el tiempo $t+\delta t$. Es importante tener en cuenta que la fuerza se asume como constante durante el paso de integración. Las fuerzas sobre las partículas en sus posiciones en el tiempo $t+\delta t$ son nuevamente determinadas comenzando con el ciclo una vez más. Como resultado, se genera una 
trayectoria que especifica cómo las posiciones y las velocidades de las partículas varían con el tiempo. Se trata de un método determinista, es decir, las posiciones de los átomos se suceden en la escala temporal y las interacciones entre las partículas son descriptas por el campo de fuerza. Existen distintos campos de fuerzas aplicados a la DM que, por lo general, se combina junto a un paquete de programas que incluyen un integrador de las ecuaciones de movimiento y herramientas de análisis.

Otras especificaciones que se deben tener en cuenta al encarar la simulación de un sistema son las condiciones de contorno. La condición más simple es que dicho sistema esté aislado. Sin embargo, esta condición -aunque puede ser parcialmente válida para sistemas gaseosos- produce efectos generalmente indeseados en sistemas moleculares sólidos o líquidos, ya que átomos en contacto con el vacío estarán afectados por fuerzas diferentes a las que existen en el sistema en las condiciones reales, produciendo una distorsión de la superficie de energía potencial. Por esta razón, se introducen condiciones periódicas de contorno; esto significa que el sistema está determinado por una celda unidad que se replica periódicamente en el espacio. Tanto la celda unidad como sus imágenes son consideradas en el cálculo.

Si la interacción se extiende sin límites es posible que una partícula sea afectada dos veces por otra, la "real" y su imagen. Para evitar esta situación artificial, se establece un radio de corte $(\mathrm{RC})$ para el potencial, que limita el alcance de la interacción. Las interacciones tipo van der Waals de a pares que están a una distancia mayor al RC son consideradas nulas y solo se consideran aquellas con las imágenes más cercanas. Esto disminuye el costo computacional lo cual es necesario para sistemas moleculares de muchos átomos, ya que la cantidad de términos no covalentes que deben ser evaluados dependen del cuadrado del número de átomos del sistema. Sin embargo, para las interacciones electrostáticas, esto no es posible porque son interacciones de muy largo alcance, incluso involucrando interacciones con átomos de la celda y sus imágenes. Por lo tanto, para este tipo de interacción se requieren metodologías eficientes para su cálculo, por ejemplo el método de sumatoria de Ewald o campo de reacción, y valores (o funciones) de corte elegidos cuidadosamente [Leach, 2001].

Además del campo de fuerzas, el RC y el método de cálculo de energías electrostáticas, en la DM se requiere elegir el tipo de ensamble, esto es, un conjunto ideal de configuraciones de sistema en el mismo estado termodinámico pero con diferentes estados dinámicos de las partículas que lo constituyen. Esta colección de configuraciones 
representa una muestra apropiada de todos los estados microscópicos y, por lo tanto, sus propiedades promedio son las del sistema promedio ideal.

Distintas restricciones macroscópicas derivan en diferentes tipos de ensambles, con características particulares. En general, se definen cuatro ensambles termodinámicos importantes:

(i) ensamble micro canónico $(\mathrm{N}, \mathrm{V}, \mathrm{E})$ : el sistema no intercambia energía ni materia con el ambiente. Su número de partículas, volumen y energía permanecen constantes;

(ii) ensamble canónico $(\mathrm{N}, \mathrm{V}, \mathrm{T})$ : el sistema intercambia energía térmica con los alrededores, pero no materia. El número de partículas, volumen y temperatura son constantes;

(iii) ensamble gran canónico $(\mu, \mathrm{V}, \mathrm{T})$ : el sistema intercambia materia y energía con el ambiente. Su potencial químico, volumen y temperatura son constantes en el tiempo; $y$

(iv) ensamble isotérmico-isobárico $(\mathrm{N}, \mathrm{T}, \mathrm{P})$ : el sistema intercambia energía térmica con los alrededores, pero no materia. Su número de partículas, temperatura y presión son constantes.

Las simulaciones computacionales de este capítulo se realizaron empleando el paquete LAMMPS [Plimpton, 1995], para estudiar los siguientes sistemas:

1) La estructura de la intercapa de Wy intercalada con ODTMA. Se construyó una celda $5 a \times 3 b \times 1 c$ con una carga total $=-12$ el. La composición de la lámina fue $\left[\left(\mathrm{Si}_{117} \mathrm{Al}_{3}\right)\left(\mathrm{Al}_{51} \mathrm{Mg}_{9}\right) \mathrm{O}_{300}(\mathrm{OH})_{60}\right]^{-12}$, el grupo espacial C2/m [Tsipursky y Drits, 1984] y los parámetro de red empleados los siguientes: $a=0,521 \mathrm{~nm} ; b=0,902 \mathrm{~nm} \mathrm{y} c=0,197 \mathrm{~nm}$. El valor del parámetro $c$, correspondiente al espaciado basal, fue tomado de los datos obtenidos por DRX, siendo su valor igual antes y después de la adsorción de TBZ. En el modelo empleado se ubicaron 12 cationes ODTMA en el espacio interlaminar. Por lo general, una simulación está conformada por una etapa de preparación, que involucra, entre otras cosas, un relajamiento del sistema a partir de optimizaciones de geometría. En el presente estudio, el primer paso consistió en una minimización de energía de la conformación inicial del modelo utilizando el campo de fuerzas paff-interface [Heinz et al., 2013]. Luego se realizó una DM en un ensamble isotérmico-isobárico (N, T, P) con presión externa de $10^{5} \mathrm{~Pa}$ y temperatura de $298 \mathrm{~K}$. Todas las posiciones atómicas en los modelos pudieron variar durante la dinámica. El paso de integración $(\delta t)$ fue 1 fs y en total se realizaron $1.10^{6}$ pasos (1 ns). Las configuraciones se colectaron cada 1000 pasos (10 ps). La 
energía electrostática se calculó empleando el método particle-particle-particle mesh (PPPM) [Leach, 2001] con una exactitud de $10^{-6} \mathrm{kcal} / \mathrm{mol}$, la energía de van der Waals fue determinada empleando el potencial de Lennard-Jones con un RC de 0,12 nm.

2) El arreglo de cationes ODTMA en la superficie externa de Wy y el arreglo de TBZ en la superficie de Wy y OWy. Se creó una caja de simulación con dimensiones de $5 a \mathrm{x} 3 b \mathrm{x}$ $1 c$, con $c=10 \mathrm{~nm}$ en el caso de OWy y TBZ-Wy. La caja de simulación consistió en dos capas T:O:T (cada una con carga de -12 el., una de ella se tomó como superficie externa con seis cationes $\mathrm{Na}^{+}$para compensar la carga) con un espacio interlaminar conteniendo las correspondientes especies (moléculas de agua y $\mathrm{Na}^{+}$en caso de $\mathrm{Wy}_{\mathrm{y}} \mathrm{TBZ}$ y cationes ODTMA en el caso de OWy). La geometría inicial del espacio interlaminar correspondió a aquellos modelos que se optimizaron en el punto 1. Los cálculos fueron desarrollados en tres etapas. En la primera, la caja de simulación contenía sólo capas T:O:T con su espacio interlaminar conteniendo las especies correspondientes $\left(\mathrm{Na}^{+}\right.$en el caso de Wy, ODTMA en el caso de $\mathrm{OWy}$ ) y moléculas de agua que llenaban el resto del volumen (densidad del agua en la caja de simulación $=1000 \mathrm{~kg} / \mathrm{m}^{3}$ ). Luego de optimizar la geometría de esos modelos, en una segunda etapa, una parte de las moléculas de agua y cationes $\mathrm{Na}^{+}$de la superficie externa fueron reemplazadas por un número estimado de moléculas de surfactante (6 cationes ODTMA y 3 pares iónicos ODTMA-Br para alcanzar el $150 \% \mathrm{CIC}$ ). En los modelos, se colocaron las cadenas hidrocarbonadas de forma perpendicular a la capa externa de la $\mathrm{Wy}$, con las cabezas cargadas cerca de la lámina T:O:T y se optimizaron estos modelos. En una tercera etapa, se evaluó un set de modelos donde la molécula de TBZ se situó en los sistemas optimizados en la etapa previa, con distintas orientaciones respecto de las láminas y de las cadenas hidrocarbonadas. Las simulaciones se realizaron empleando la molécula neutra y la molécula protonada. La geometría de estos modelos fue optimizada en un ensamble canónico $(\mathrm{N}, \mathrm{V}, \mathrm{T})$ con todas las posiciones atómicas variables. El paso de integración $(\delta t)$ fue 1 fs y se llevaron a cabo entre $1.10^{6}$ y $2.10^{6}$ pasos (entre 1 y 2 ns). Las configuraciones del sistema se colectaron cada 10 ps. La energía electrostática se calculó utilizando el método PPPM con una exactitud de $10^{-6} \mathrm{kcal} / \mathrm{mol}$ y la energía van der Waals fue calculada empleando el potencial de Lennard-Jones con un RC de 0,12 nm.

\subsubsection{Estudios de adsorción-desorción}

Se determinaron isotermas de adsorción de TBZ en las muestras seleccionadas. Las isotermas se realizaron en batch empleando tubos de plástico, relación sólido/solución $=1$ $\mathrm{g} / \mathrm{L}$, intervalo de concentraciones de TBZ de 0,5-25 mg/L, tiempo de contacto de $24 \mathrm{~h}$ a 
$25{ }^{\circ} \mathrm{C}$ en agitación constante, sin ajustes de $\mathrm{pH}$. El tiempo y las condiciones se eligieron teniendo en cuenta los estudios reportados en la bibliografía y resumidos en la introducción de este capítulo [Lombardi, 2004; Roca Jalil et al., 2013; 2014]. A $t=0$, el pH fue 6,5 en todas las muestras; mientras que a $t_{e q}=24 \mathrm{~h}$, el $\mathrm{pH}$ fue 7,5 para Mt; 7 para OMt55, OMt137 y OWy y 8 para Wy.

Luego del tiempo de equilibrio, las suspensiones se centrifugaron a $14.000 \mathrm{rpm}$ durante 15 min. La concentración de TBZ en los sobrenadantes se determinó mediante cromatografía líquida de alta resolución (HPLC) acoplado con un detector UV-visible $(\lambda=$ $298 \mathrm{~nm})$ y fluorescencia $\left(\lambda_{e x}=300 \mathrm{~nm} ; \lambda_{e m}=350 \mathrm{~nm}\right)$. Se usó un equipo Shimadzu con columna C18 (4,6 mm x $250 \mathrm{~mm}, 4,6 \mu \mathrm{m})$. La fase móvil fue metanol/agua (70/30) fluyendo a $1 \mathrm{~mL} / \mathrm{min}$. El volumen inyectado fue de $10 \mu \mathrm{L}$. Los límites de detección (LD) y de cuantificación $(\mathrm{LC})$ fueron $\mathrm{LD}=0,0037 \mathrm{mg} / \mathrm{L}$ y $\mathrm{LC}=0,0045 \mathrm{mg} / \mathrm{L}$ para $\mathrm{UV}$, y $\mathrm{LD}=$ 0,00086 mg/L y LC = 0,00052 mg/L para FL [Suárez Pérez et al., 2009]. La cantidad adsorbida de TBZ en el equilibrio $(Q)$ se determinó empleando la Ecuación 3.3.

En todas las isotermas, se ajustaron a los datos experimentales las ecuaciones de Langmuir y Freundlich (Sección 3.2.3). Además, se evaluó el modelo matemático Langmuir-Freundlich (LF), una ecuación de tres parámetros que fue propuesta para evitar la imposibilidad encontrada en Freundlich de alcanzar un plateau a concentraciones finales altas. El modelo LF está representado por

$$
Q=\frac{Q_{\max , S}\left(K_{S} C_{f}\right)^{\frac{1}{n}}}{1+\left(K_{S} C_{f}\right)^{\frac{1}{n}}}
$$

donde $K_{S}[\mathrm{~L} / \mathrm{mg}]$ es la constante de afinidad, $Q_{\max , S}[\mathrm{mg} / \mathrm{g}]$ es la máxima capacidad de adsorción del adsorbente y $1 / n$ (adimensional) es el parámetro de Freundlich que caracteriza la heterogeneidad de la superficie [Kinniburgh, 1986]. La Ecuación 4.2 se reduce a la expresión de Langmuir cuando $1 / n=1$, es decir, cuando la superficie es energéticamente homogénea. Mientras más se aleje este parámetro de la unidad, mayor es la heterogeneidad de la superficie [Guimarães et al., 2016].

Se realizaron estudios de desorción sobre las muestras TBZ-Mt, TBZ-OMt55, TBZOMt137, TBZ-Wy y TBZ-OWy, para obtener información acerca de la fuerza de unión entre el fungicida y el adsorbente. Para ello, las suspensiones obtenidas con la solución más concentrada de TBZ $\left(C_{i}=25 \mathrm{mg} / \mathrm{L}\right)$ se centrifugaron luego del tiempo de equilibrio, y posteriormente se reemplazó la mitad del volumen del sobrenadante por agua, se re- 
equilibraron las nuevas suspensiones por $24 \mathrm{~h}$, y se repitió todo el procedimiento dos veces más.

\subsection{Resultados y discusión}

\subsubsection{Caracterización de los adsorbentes}

Las curvas Tg, DTg y ATD de Wy y OWy se muestran en la Figura 4.1; mientras que la Tabla 4.1 presenta las regiones, pasos de descomposición térmica, pérdidas de masa y temperaturas de los picos DTg y ATD.
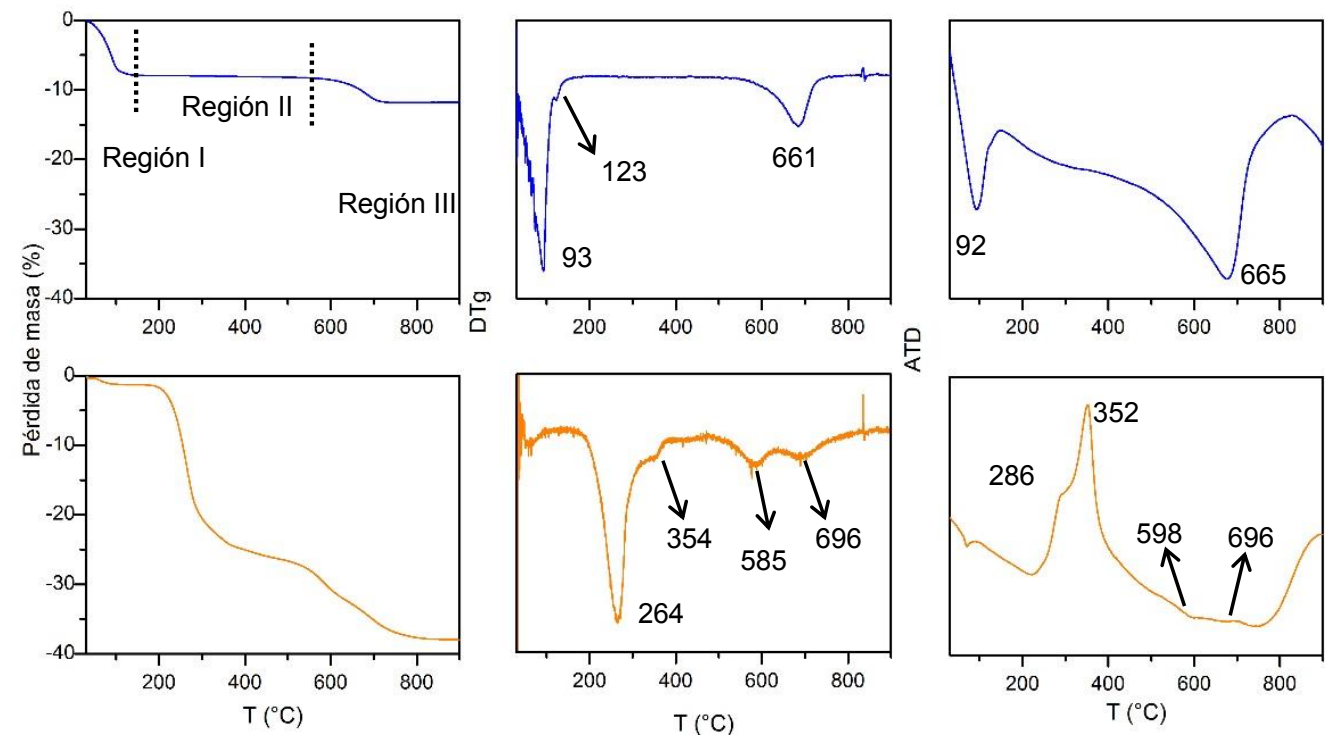

Figura 4.1 Curvas de análisis térmicos. De izquierda a derecha: Tg, DTg y ATD de (一) Wy y ( - OWy.

Wy presentó dos importantes etapas de pérdida de masa en $\mathrm{Tg}$, con picos ATD endotérmicos asociados a la deshidratación de la muestra. En la Región I (25-170 $\left.{ }^{\circ} \mathrm{C}\right)$ un importante pico endotérmico a $92{ }^{\circ} \mathrm{C}$ correspondió a la desorción de agua fisisorbida, y estuvo asociado a un pico DTg a $93{ }^{\circ} \mathrm{C}$. En este intervalo se encontró también un pico DTg a $123{ }^{\circ} \mathrm{C}$ producido por la deshidratación de los cationes interlaminares. En conjunto, ambos procesos condujeron a la pérdida de un 7,9\% de la masa inicial de la muestra.

El intercambio con ODTMA provocó el corrimiento de los picos DTg y ATD correspondientes a la desorción de agua fisisorbida hacia menores temperaturas (Tabla 4.1, Figura 4.1), indicando que el agua residual se unió débilmente a la superficie hidrofóbica de la muestra $\mathrm{OWy}$, ya que probablemente las moléculas de agua se encuentran en los poros entre las cadenas hidrofóbicas y no directamente unida a los grupos hidroxilos de la arcilla [Hedley et al., 2007]. Estas observaciones confirmaron la presencia de moléculas orgánicas en la superficie externa de las muestras intercambiadas, que más adelante en esta sección se 
analizará también mediante XPS. Además, y al igual que las organo-montmorillonitas obtenidas en el Capítulo 3, el pico asociado a la deshidratación de los cationes interlaminares está ausente en OWy, debido al intercambio de los cationes inorgánicos interlaminares por la amina cuaternaria. Más aún, el porcentaje de pérdida de masa en la Región I fue menor en la muestra OWy (1,3\%), confirmando el incremento de hidrofobicidad debido a la presencia de las cadenas alquílicas de ODTMA.

Tabla 4.1 Reacciones térmicas, picos DTg y ATD $\left[{ }^{\circ} \mathrm{C}\right]$ y porcentajes de pérdida de masa $[\%]$ de Wy y OWy.

\begin{tabular}{|c|c|c|c|c|}
\hline & & & Wy & OWy \\
\hline \multirow[t]{6}{*}{ Región I } & \multicolumn{2}{|c|}{ Intervalo de $\mathrm{T}$} & $30-170$ & $30-115$ \\
\hline & Desorción de agua fisisorbida & DTg & 93 & 61 \\
\hline & & ATD (endo) & 92 & 72 \\
\hline & $\begin{array}{l}\text { Deshidratación de cationes } \\
\text { interlaminares }\end{array}$ & DTg & 123 & n.d. \\
\hline & & ATD (endo) & n.d. & n.d. \\
\hline & Pérdida de masa total & $\mathrm{Tg}$ & 7,9 & 1,3 \\
\hline \multirow[t]{7}{*}{ Región II } & \multicolumn{2}{|c|}{ Intervalo de $\mathrm{T}$} & n.c. & $160-381$ \\
\hline & \multirow[t]{2}{*}{$\begin{array}{l}\text { 1er Paso de oxidación de contenido } \\
\text { orgánico. }\end{array}$} & DTg & n.c. & $264 ; 354(h)$ \\
\hline & & ATD (exo) & n.c. & 286 (h); 352 \\
\hline & \multicolumn{2}{|c|}{ Intervalo de $\mathrm{T}$} & n.c. & $381-474$ \\
\hline & $\begin{array}{l}\text { 2do Paso de oxidación de contenido } \\
\text { orgánico. }\end{array}$ & & n.c. & 428 (h) \\
\hline & & ATD (exo) & n.c. & n.d. \\
\hline & Pérdida de masa total & $\mathrm{Tg}$ & 0,3 & 28,0 \\
\hline \multirow[t]{6}{*}{ Región III } & \multicolumn{2}{|c|}{ Intervalo de $\mathrm{T}$} & $528-767$ & $474-851$ \\
\hline & Deshidroxilación & DTg & 661 & 585 \\
\hline & & ATD (endo) & 665 & 598 \\
\hline & Oxidación de restos carbónicos & DTg & n.c. & 696 \\
\hline & & ATD (exo) & n.c. & 696 \\
\hline & Pérdida de masa total & $\mathrm{Tg}$ & 3,6 & 11,6 \\
\hline
\end{tabular}

n.c.: no corresponde; n.d.: no determinado; (h): hombro

En la Región II, Wy no presentó picos ATD ni DTg aunque registró en Tg una pérdida de masa del 0,3\%. La muestra OWy, en cambio, mostró dos pasos de oxidación en esta región. El primer paso de oxidación ocurrió entre $160-381{ }^{\circ} \mathrm{C}$ con el pico DTg más 
importante centrado a $264^{\circ} \mathrm{C}$. Éste se asoció a la oxidación de $\mathrm{H}$ de las cadenas alquílicas a $\mathrm{H}_{2} \mathrm{O}$, con formación de carbón [Keidar et al., 2015]. El segundo paso de oxidación, ocurrió entre $381-474{ }^{\circ} \mathrm{C}$, con un pico DTg (menor) a $428{ }^{\circ} \mathrm{C}$ y sin picos ATD. En este intervalo de temperaturas ocurrió la combustión del carbón estable a bajas temperaturas a $\mathrm{CO}_{2}$ y $\mathrm{NO}_{2}$ y la formación de carbón estable a altas temperaturas. La pérdida de masa total en la Región II fue de 28,0\%.

A partir de este porcentaje, se calculó el contenido de ODTMA en la muestra OWy según la Ecuación 4.5:

$$
X=\frac{\left(W \times 10^{5}\right)}{\operatorname{CIC} x(M-y) \times(100-W)}
$$

donde $X$ es el contenido de surfactante de la muestra [meq/g]; $M$ la masa molecular del surfactante $[\mathrm{g} / \mathrm{mol}]$; $W$ el porcentaje de pérdida de masa en la Región II [\%]; CIC es la capacidad de intercambio catiónico [meq/100 g]; $y$ puede tomar dos valores: 0 (cero) si todos los iones $\mathrm{Br}^{-}$permanecen o $\mathrm{M}(\mathrm{Br})=79,9 \mathrm{~g} / \mathrm{mol}$ (masa atómica del $\mathrm{Br}$ ) si no hay iones $\mathrm{Br}^{-}$presentes en la muestra. $\mathrm{El}$ hecho de que $y$ no pueda tomar valores intermedios permite estimar valores teóricos máximos y mínimos de ODTMA en la muestra, y no un valor exacto [Boeva et al., 2013]. Este cálculo teórico indicó que el contenido de ODTMA en la muestra OWy estuvo entre el 115 y el $140 \%$ CIC.

En la Región III ocurren tanto la deshidroxilación como el último paso de oxidación de la materia orgánica (combustión de carbón estable a altas temperaturas). La deshidroxilación de $\mathrm{W}_{y}$, condujo a la pérdida de 3,6\% de masa y a la aparición de picos ATD $\left(665^{\circ} \mathrm{C}\right.$, endotérmico) y DTg $\left(661^{\circ} \mathrm{C}\right)$. La incorporación de ODTMA produjo una disminución de la temperatura del pico endotérmico hacia $585{ }^{\circ} \mathrm{C}$ y la aparición de un nuevo pico, exotérmico, a $696{ }^{\circ} \mathrm{C}$ asociado al último paso de oxidación del contenido orgánico. La pérdida de masa total en OWy en la Región III fue de 11,6\%.

La Figura 4.2 muestra los difractogramas de Wy y OWy. La muestra Wy presentó un pico de reflexión $\mathrm{d}_{001}$ correspondiente a un espaciado basal de 1,26 nm, similar al encontrado para la muestra Mt en el Capítulo 3. Este espaciado basal fue asignado a la esfera de hidratación de los cationes $\mathrm{Na}^{+}$presentes en la intercapa. El intercambio de éstos con ODTMA provocó el corrimiento del pico $\mathrm{d}_{001}$ hacia menores ángulos, lo cual correspondió a un espaciado basal de 1,99 nm. Xi et al. [2007] concluyeron que un espaciado basal de 1,90 nm de una octadeciltrimetilamonio-montmorillonita correspondía a un arreglo bicapa de las cadenas alquílicas, mientras que un espaciado basal de 2,1 nm con un hombro a 1,95 $\mathrm{nm}$ reflejaba una transición del arreglo bicapa al arreglo tipo parafina. 
Estas asunciones se basaron en el cálculo del tamaño molecular de una molécula de ODTMA (a partir de los radios de van der Waals, distancias de enlace covalente y ángulo diedro) y considerando el espesor de una lámina T:O:T de montmorillonita igual a 0,97 nm. Teniendo esto en cuenta, el espaciado basal aquí encontrado, se atribuyó a una transición entre un arreglo tipo bicapa y un arreglo tipo parafina.

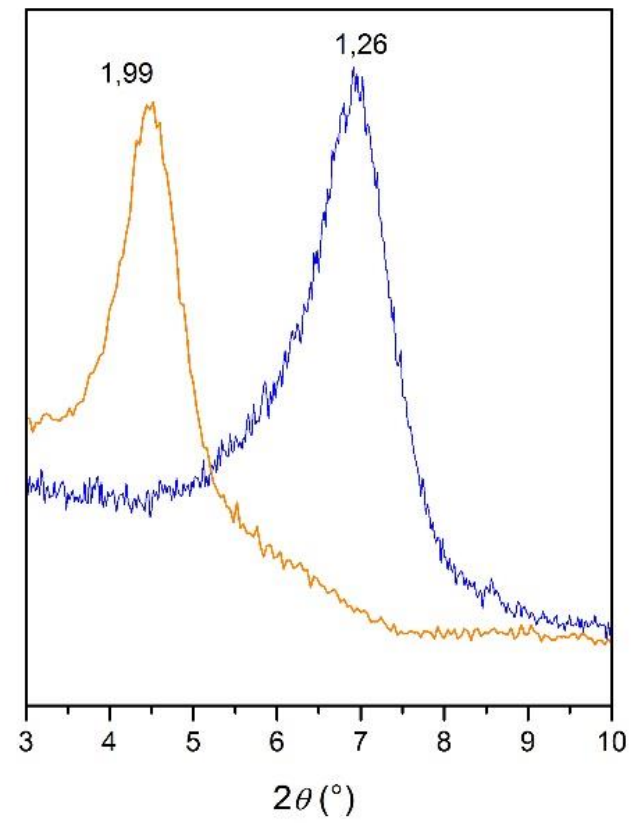

Figura 4.2 Difractogramas con el espaciado basal indicado (en nm) de (-) Wy y (-) OWy.

La simulación computacional de la intercapa de OWy empleando un espaciado basal de 1,99 nm y 12 cationes ODTMA condujo a un empaquetamiento denso de las cadenas, que adoptaron una orientación paralela a las lámina T:O:T (Figura 4.3). Los cationes ODTMA saturaron el espacio interlaminar, sin dejar espacios libres (como puede apreciarse en la Figura 4.3 (abajo) donde se representan los radios de van der Waals de los átomos). La $\mathrm{DM}$, en la cual se permitió que el parámetro $c$ modifique su valor, condujo a un espaciado basal en un rango de 1,90 $\mathrm{nm}$ a 2,10 $\mathrm{nm}$. El primero correspondió a un arreglo bicapa (Figura 4.3 arriba, izquierda). En el segundo, el arreglo inicial tipo bicapa fue parcialmente interrumpido (Figura 4.3 arriba, derecha); las cabezas cargadas de los surfactantes se colocaron cercanas a las láminas negativas de la arcilla. Esa distorsión de las cadenas ODTMA formaron un arreglo "pseudo-tricapa" de acuerdo a lo determinado por Xi et al. [2007].

Las curvas de potencial zeta vs. $\mathrm{pH}$ de las muestras Wy y OWy (Figura 4.4) son similares a las de las muestra Mt y OMt137 (Figuras 3.2 y 3.3). OWy presentó carga superficial positiva en todo el intervalo de pH estudiado, indicando que el ODTMA se unió 
tanto en los sitios de carga permanente como en los sitios de superficie externa de la montmorillonita. Esto es coherente con la estimación de contenido orgánico en la muestra.
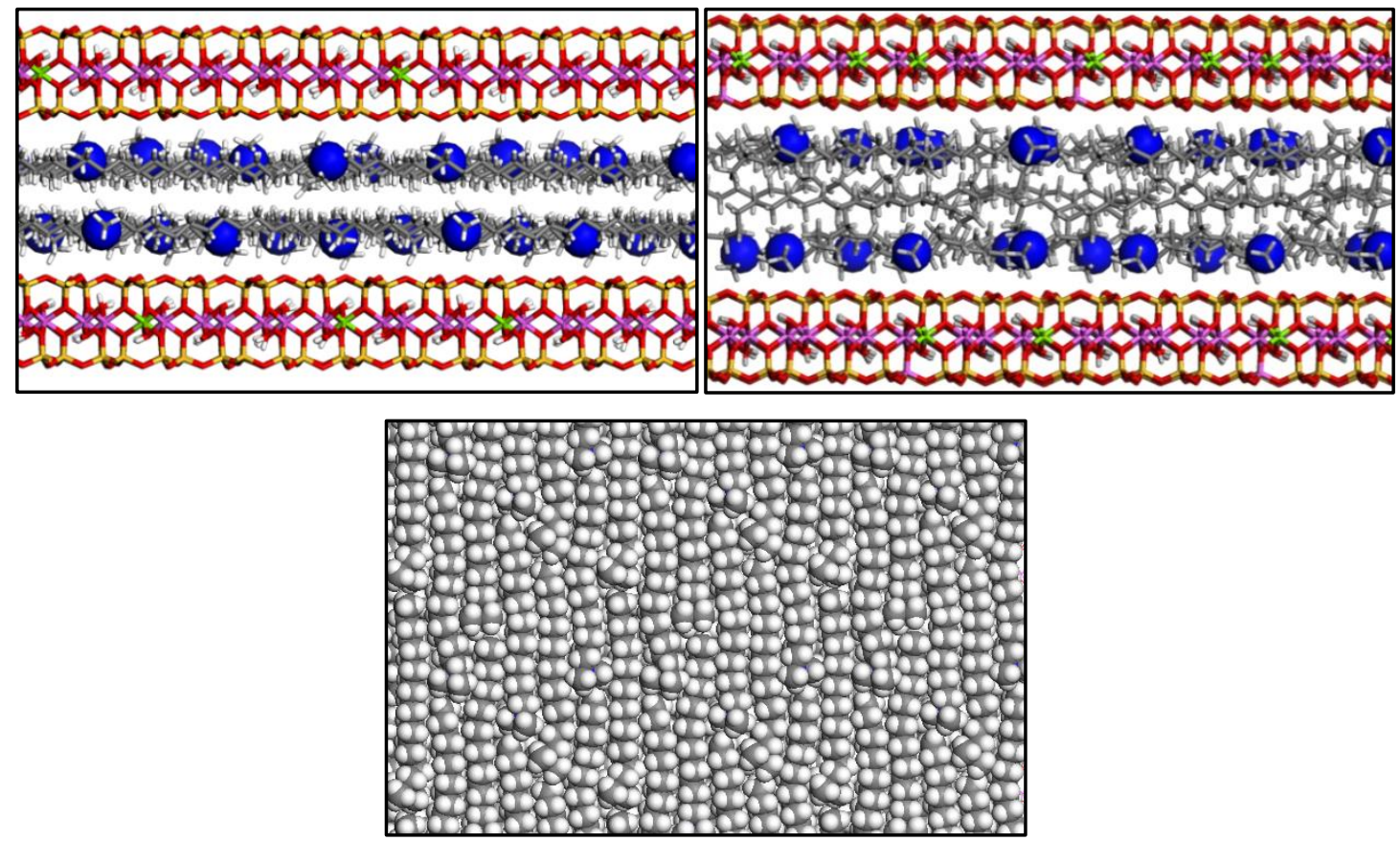

Figura 4.3 Arreglo de ODTMA en la intercapa de OWy. Arriba, izquierda: bilaminar (0,19 nm). Arriba, derecha: pseudo tricapa $(0,21 \mathrm{~nm})$. Abajo: empaquetamiento de las cadenas desde un punto de vista cenital. Las esferas representan los radios de van der Waals.

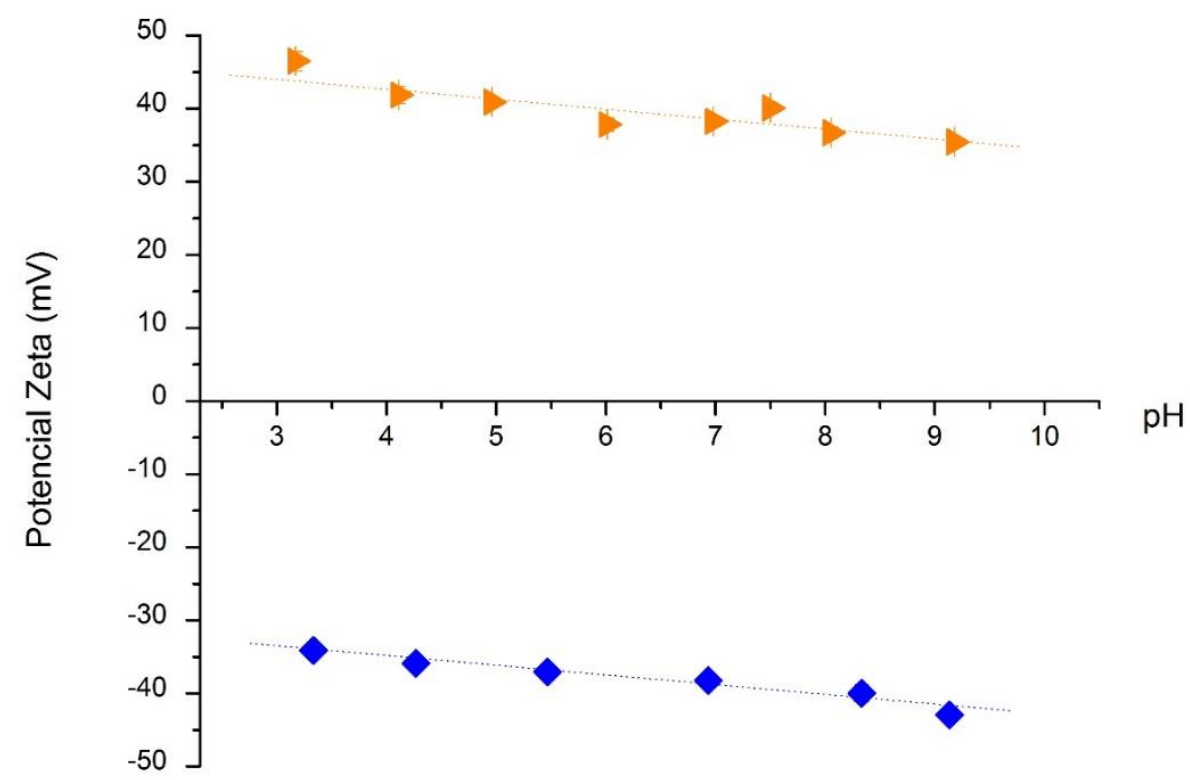

Figura 4.4 Curvas de potencial zeta vs. pH de $(\downarrow)$ Wy y $(\triangleright)$ OWy. Las curvas son regresiones lineales utilizando una ecuación polinomial, con el objetivo de facilitar el seguimiento de los puntos correspondientes a cada muestra. 
La composición elemental en la superficie externa de estas muestras fue determinada mediante XPS (Tabla 4.2). En estos análisis el área de la señal correspondiente a un elemento está relacionada con el contenido de dicho elemento en la superficie de la muestra y éste se suele expresar como porcentaje molar del elemento respecto a la totalidad de los elementos analizados. La descomposición matemática de la señal de XPS de un elemento permite determinar su especiación en superficie. En este sentido, el área de los picos obtenidos mediante la descomposición matemática está relacionada con el contenido de la especie respectiva en superficie.

Tabla 4.2 Composición elemental de la superficie externa determinada por XPS (fracciones molares, \%).

\begin{tabular}{|c|c|c|c|}
\hline & ODTMA & Wy & OWy \\
\hline $\mathrm{Na} 1 s$ & - & 1,3 & - \\
$\mathrm{Si} 2 p$ & - & 17,9 & 9,2 \\
$\mathrm{O}_{\text {TOTAL }}$ & 5,9 & 49,6 & 28,9 \\
$\mathrm{C} 1 s(285 \mathrm{eV})$ & 54.2 & 15,9 & 43,1 \\
$\mathrm{C} 1 s(286,6 \mathrm{eV})$ & 30,4 & 3,6 & 8,7 \\
$\mathrm{C} 1 s(289 \mathrm{eV})$ & 2,0 & 2,0 & 2,2 \\
$\mathrm{C}_{\text {TOTAL }}$ & 86,6 & 21,5 & 54,0 \\
$\mathrm{Br} 3 d$ & 2,4 & - & 0,7 \\
$\mathrm{Br} 3 d / \mathrm{C}_{\text {TOTAL }}$ & 0,028 & - & 0,013 \\
$\mathrm{Br} 3 d / \mathrm{Si} 2 p$ & - & - & 0,076 \\
$\mathrm{~N} 1 s(399 \mathrm{eV})$ & 0,6 & - & 0,4 \\
$\mathrm{~N} 1 s(402 \mathrm{eV})$ & 2,6 & - & 1,6 \\
$\mathrm{~N} 1 s(402 \mathrm{eV}) /(399 \mathrm{eV})$ & 4,3 & - & 4,0 \\
$\mathrm{~N}_{\text {TOTAL }}$ & 3,2 & - & 2,0 \\
$\mathrm{Al}_{2} p$ & - & 7,0 & 3,5 \\
$\mathrm{Mg}_{\text {KLL }}$ & 1,9 & 2,7 & 1,6 \\
\hline
\end{tabular}

La presencia de cationes $\mathrm{Na}^{+}$en la muestra Wy (Tabla 4.2) fue confirmada por la presencia del pico a 1073,2 eV correspondiente al electrón $1 s$ de este elemento. La ausencia de este pico en la muestra OWy evidenció que el catión $\mathrm{Na}^{+}$fue totalmente intercambiado por el catión orgánico en la superficie externa, y nuevamente, es coherente con los 
resultados del cálculo de Boeva que indicaron que el surfactante se intercambió en un porcentaje mayor al $100 \%$ CIC.

Las bandas asignadas a $\mathrm{Si} 2 p$ y $\mathrm{O} 1 s$, constituyentes de la estructura del aluminosilicato [Seyama y Soma, 1985], se corrieron hacia menores energías de enlace desde 103,4 a 102,6 eV y desde 532,7 a 531,8 eV, cuando se intercaló OTDMA. La disminución de las energías de enlace de $\mathrm{Si} 2 p$ y O1s estuvieron de acuerdo con los resultados obtenidos por He et al. [2007] para una hexadeciltrimetilamonio-montmorillonita, reflejando el cambio en el entorno, hidrofílico en $\mathrm{W}_{y}$ a hidrofóbico en $\mathrm{OW}_{\mathrm{y}}$, por el apantallamiento del núcleo ${ }^{29} \mathrm{Si}$ [Breakwell et al., 1995].

El espectro de alta resolución de la región del pico C1s para las muestras presentó componentes a 285; 286,6 y $289 \mathrm{eV}$, correspondientes a los enlaces $\mathrm{C}-\mathrm{C}, \mathrm{C}-\mathrm{N}$ o $\mathrm{C}-\mathrm{O}$ y COOR, respectivamente (Figura 4.5). El incremento de $\mathrm{C}_{\text {TOTAL }}$ en fracciones molares en la muestra OWy respecto a Wy (Tabla 4.2), fue otra evidencia de la presencia de las cadenas alquílicas del surfactante en la superficie de la organo-montmorillonita. La banda asignada a COOR en Wy y en OWy se mantuvo prácticamente constante debido a que su origen es la contaminación de la muestra durante el experimento (notar que el porcentaje es similar en Wy y OWy (Tabla 4.2) y que el ODTMA no presenta este tipo de grupos funcionales) [Briggs y Beamson, 1992].
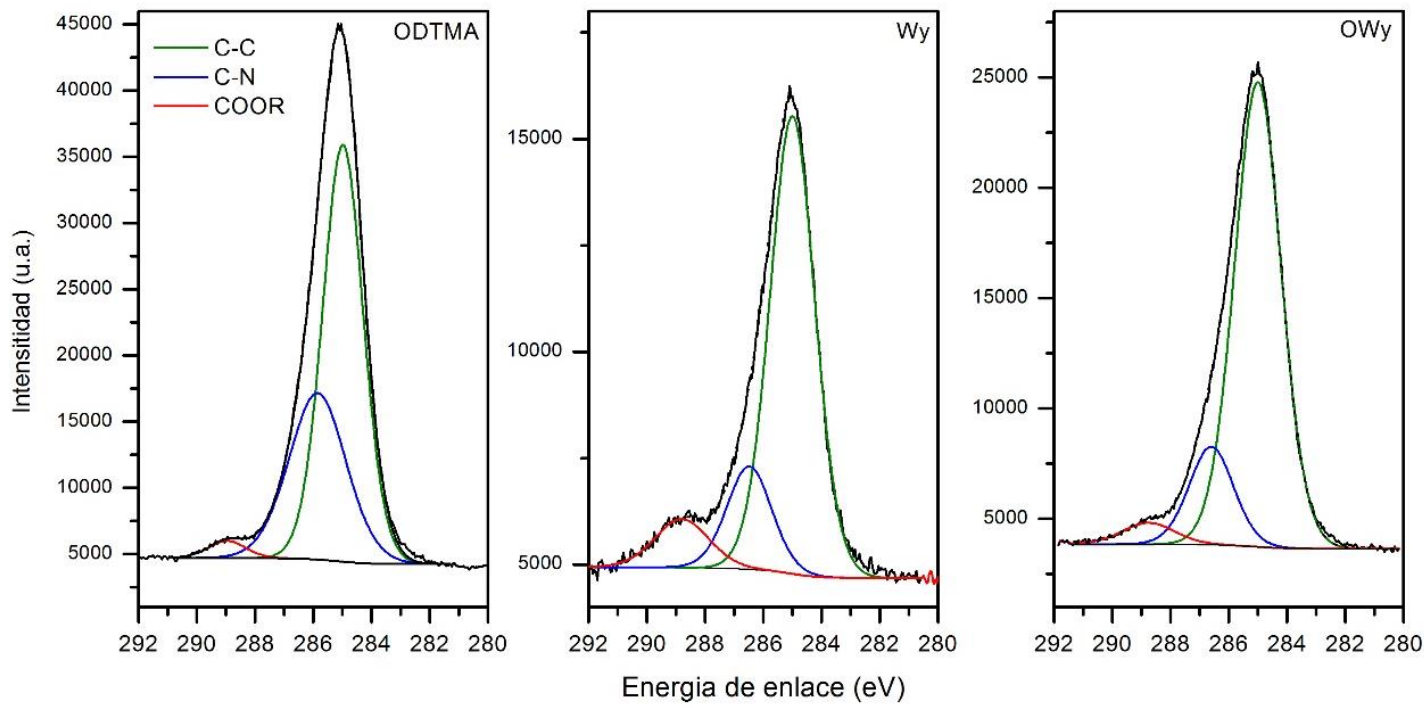

Figura 4.5 Espectros de alta resolución de la banda correspondiente al electrón C1s de las muestras indicadas.

Otro dato importante es la presencia de $\mathrm{Br}^{-}$en la muestra OWy. Mientras que ODTMA presenta una relación $\mathrm{Br} 3 d / \mathrm{C}_{\mathrm{TOTAL}}=0,028$ (siendo 0,020 menor a la relación estequiométrica debido al carbono proveniente de la contaminación) la muestra OWy 
presentó una relación $\mathrm{Br} 3 d / \mathrm{C}_{\text {TOTAL }}=0,013$, correspondiendo al 46,8\% del valor de ODTMA puro. Esto sugiere que sólo algunas moléculas ODTMA-Br son adsorbidas como pares iónicos, mediante fuerzas de van der Waals (o interacciones hidrofóbicas) en la superficie externa de la Mt [Lee et al., 2005] y se corresponde con los resultados de potencial zeta y con la estimación teórica del contenido de ODTMA. Este resultado podría extrapolarse a la muestra OMt137, que presenta similar contenido orgánico, espaciado basal y carga superficial. El anión $\mathrm{Br}^{-}$neutraliza (en parte) las cargas positivas externas en el sólido, pero cuando la muestra se suspende, el anión se ubica en la capa de difusión en la doble capa eléctrica y por ello no es detectado en los experimentos de microelectroforesis.

En las muestras, ODTMA y OWy el pico N1s se descompuso en dos componentes (Figura 4.6, Tabla 4.2) localizadas a 399,7 y 402,4 eV. Estos picos se atribuyeron a átomos unidos a través de diferentes energías de enlace (uniones electrostáticas fuertes y uniones de van der Waals débiles, respectivamente) apoyando la interpretación de párrafos previos [Naranjo et al., 2013].

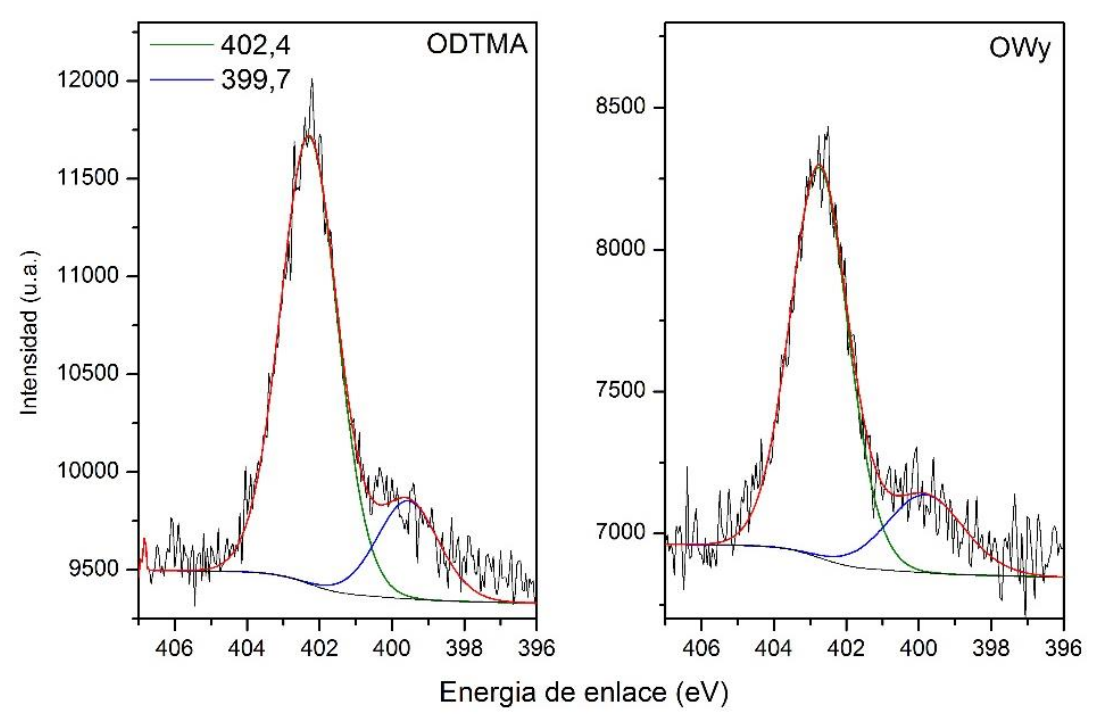

Figura 4.6 Espectros de alta resolución de la banda correspondiente al electrón N1s de las muestras indicadas.

\subsubsection{Estudios de adsorción-desorción}

Las isotermas de adsorción experimentales, junto a los correspondientes ajustes de LF, se muestran en la Figura 4.5. El coeficiente de correlación $\mathrm{R}^{2}$, junto a los parámetros de los modelos de Freundlich, Langmuir y LF, se resumen en la Tabla 4.3.

El valor de $\mathrm{R}^{2}$ obtenido para la adsorción de TBZ en Mt indica que Freundlich no describe bien los datos experimentales. Por otro lado, las isotermas de adsorción del 
fungicida en las muestras con mayor contenido orgánico (OMt137 y OWy) presentaron mejores valores de $\mathrm{R}^{2}$ cuando se ajustó Langmuir, pero los parámetros presentaron errores asociados importantes y hasta más grandes que su valor absoluto. Esto evidencia que estos sistemas no cumplen las hipótesis planteadas por los modelos y para poder comparar el desempeño de los adsorbentes fue necesario buscar otra ecuación matemática. En ese sentido, el modelo LF mostró un buen ajuste a los datos experimentales y fue el seleccionado para analizar los resultados.
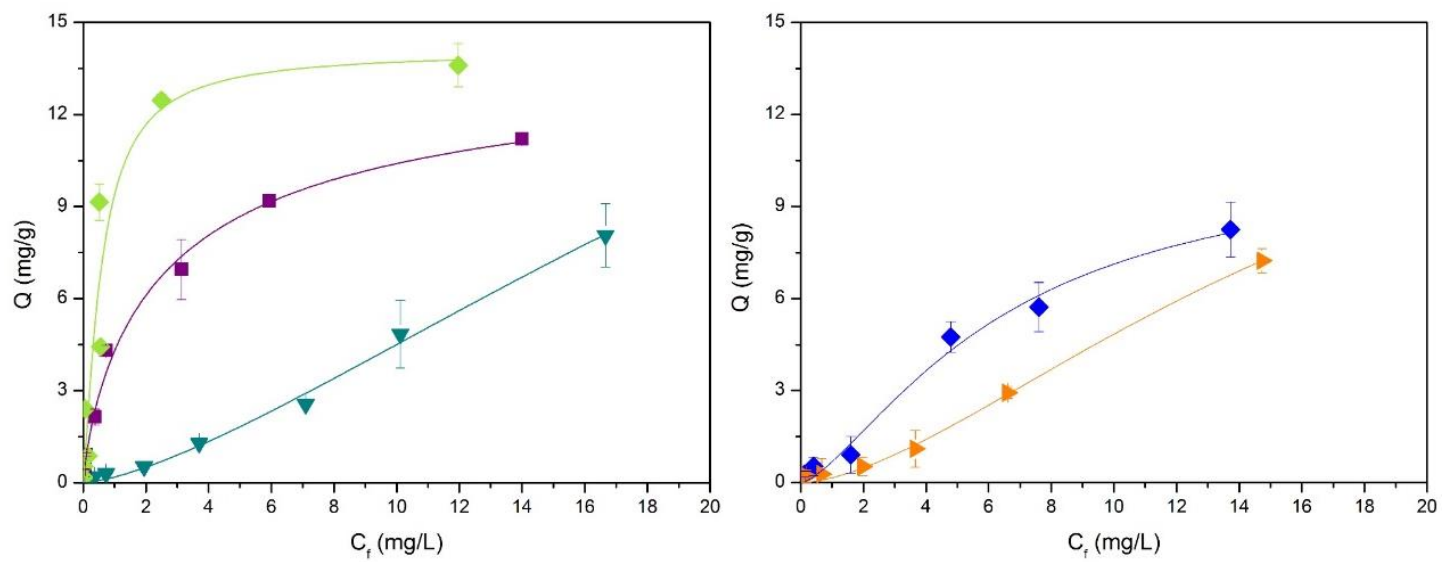

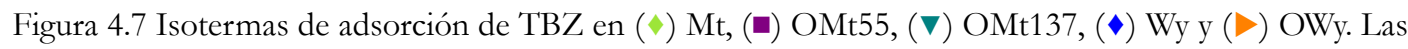
líneas sólidas corresponden al ajuste de Langmuir-Freundlich.

Tabla 4.3 Parámetros obtenidos mediante el ajuste de los modelos indicados a las isotermas de adsorción de TBZ en Mt, OMts, Wy y OWy.

\begin{tabular}{|c|c|c|c|c|c|c|}
\hline & & Mt & OMt55 & OMt137 & Wy & OWy \\
\hline \multirow{3}{*}{ Freundlich } & $K_{f}[\mathrm{~L} / \mathrm{mg}]$ & $7 \pm 1$ & $3,9 \pm 0,4$ & $0,27 \pm 0,04$ & $1,3 \pm 0,3$ & $0,27 \pm 0,04$ \\
\hline & $1 / n$ & $0,31 \pm 0,09$ & $0,42 \pm 0,05$ & $1,21 \pm 0,06$ & $0,7 \pm 0,1$ & $1,22 \pm 0,06$ \\
\hline & $\mathrm{R}^{2}$ & 0,789 & 0,958 & 0,993 & 0,965 & 0,995 \\
\hline \multirow{3}{*}{ Langmuir } & $Q_{\max }[\mathrm{mg} / \mathrm{g}]$ & $15 \pm 2$ & $12,1 \pm 0,6$ & $1.10^{5} \pm 6.10^{6}$ & $17 \pm 4$ & $2.10^{5} \pm 9.10^{6}$ \\
\hline & $k[\mathrm{~L} / \mathrm{mg}]$ & $1,5 \pm 0,5$ & $0,6 \pm 0,1$ & $3.10^{-5} \pm 9.10^{-3}$ & $0,07 \pm 0,03$ & $3.10^{-5} \pm 1.10^{-2}$ \\
\hline & $\mathrm{R}^{2}$ & 0,921 & 0,988 & 0,980 & 0,979 & 0,977 \\
\hline \multirow{4}{*}{$\mathrm{LF}$} & $Q_{\max , S}[\mathrm{mg} / \mathrm{g}]$ & $14 \pm 2$ & $14 \pm 2$ & $20 \pm 5$ & $10 \pm 3$ & $17 \pm 3$ \\
\hline & $K_{s}[\mathrm{~L} / \mathrm{mg}]$ & $1,7 \pm 0,8$ & $0,3 \pm 0,1$ & $0,03 \pm 0,03$ & $0,16 \pm 0,06$ & $0,06 \pm 0,06$ \\
\hline & $1 / n$ & $1,2 \pm 0,7$ & $0,8 \pm 0,1$ & $1,5 \pm 0,2$ & $1,5 \pm 0,4$ & $1,6 \pm 0,3$ \\
\hline & $\mathrm{R}^{2}$ & 0,920 & 0,989 & 0,994 & 0,980 & 0,995 \\
\hline
\end{tabular}

A partir de la Figura 4.7 puede verse que las organo-montmorillonitas no mejoraron la adsorción del fungicida respecto a la montmorillonita natural, en el intervalo de concentraciones de TBZ y en las condiciones de trabajo. Sin embargo, el valor de $Q_{\max , S}$ es igual en OMt55 y mayor en OMt137 que en Mt, mientras que el de OWy fue mayor al de 
Wy. Esto es resultado de la extrapolación matemática, ya que ninguna muestra -excepto Mt- alcanzó un plateau de adsorción, sugiriendo que a concentraciones más altas de fungicida en equilibrio $\left(C_{f}\right)$, los adsorbentes podrían aumentar la cantidad adsorbida por unidad de masa $(Q)$. Como esta hipótesis no puede confirmarse debido a que la máxima concentración inicial de TBZ que puede ensayarse está limitada por la solubilidad del mismo, en el Capítulo 6 se estudiará el efecto en la capacidad de remoción de Mt y OMt55 cuando se disminuye la relación sólido/solución.

La constante de afinidad $K_{s}$ disminuyó en un orden de magnitud cuando la Mt fue intercambiada con ODTMA al 55 \%CIC, y disminuyó dos órdenes de magnitud cuando fue intercambiada en un 137 \%CIC. De forma análoga, OWy mostró una constante de afinidad un orden de magnitud menor a Wy. Esto evidenció que el recubrimiento de la superficie siloxánica con cadenas alquílicas no favoreció la adsorción de TBZ. Teniendo en cuenta la naturaleza básica del TBZ, y que los sitios activos de adsorción en la Mt son los sitios de carga permanente, es esperable que una disminución en el número de sitios disponibles debido a su ocupación por cationes ODTMA, generen la disminución del valor de $Q$ que se observa en las isotermas. A medida que el contenido de catión orgánico aumentó, el número de sitios disponibles para TBZH ${ }^{+}$disminuyó. De hecho, en la muestra OWy la dinámica molecular indicó que no hay sitios de carga permanente ni sitios hidrofóbicos en la intercapa disponibles para la unión de TBZ. Esto explica que tanto esta muestra como la OMt137, presenten los valores más bajos de $\mathcal{Q}$ en todo el intervalo de $C_{f}$ estudiado. Es necesario, entonces, indagar a qué sitios se une el TBZ en estas muestras.

Para evaluar la fuerza de la interacción entre el fungicida y el sólido se llevaron a cabo experimentos de desorción. En la Tabla 4.4 se muestran la cantidad adsorbida de TBZ por unidad de masa $(Q)$ y el porcentaje que se desorbe luego de tres días respecto de esa cantidad.

Tabla 4.4 Cantidad adsorbida por unidad de masa y porcentajes de desorción de TBZ.

\begin{tabular}{|c|c|c|}
\hline Muestra & $\mathcal{Q}[\mathrm{mg} / \mathrm{g}]$ & Desorción [\%] \\
\hline TBZ-Wy & $8,2 \pm 0,9$ & 0 \\
TBZ-OWy & $7,2 \pm 0,4$ & $82 \pm 2$ \\
TBZ-Mt & $13,6 \pm 0,7$ & 0 \\
TBZ-OMt55 & $11,2 \pm 0,1$ & 0 \\
TBZ-OMt137 & $8 \pm 1$ & $81 \pm 3$ \\
\hline
\end{tabular}


No se registró desorción de TBZ en las montmorillonitas naturales (Mt y Wy) ni tampoco en la organo-montmorillonita con ODTMA al 55 \%CIC. Esto se justifica por el desarrollo de interacciones electrostáticas entre el sólido y la especie $\mathrm{TBZH}^{+}$, lo cual confirma que los sitios activos de adsorción son los de carga permanente en estas muestras. Hay que notar que esto es válido para OMt55, ya que en esta muestra hay más del 40 $\%$ CIC de sitios de carga permanente ocupados por $\mathrm{Na}^{+}$y el fungicida se encuentra adsorbido en apenas un 6,7\%CIC. En cambio, cuando el contenido de ODTMA supera el $100 \%$ CIC la desorción aumenta a más del 80\% respecto al fungicida inicial adsorbido. Es decir que son otro tipo de fuerzas las que están involucradas en la interacción entre el adsorbato y estos sólidos.

\subsubsection{Caracterización de productos de adsorción}

Los difractogramas de los productos de adsorción TBZ-Mt, TBZ-OMt55, TBZ-Wy y TBZ-OWy están representados en la Figura 4.8.

Por un lado, la intercalación de TBZ en Wy y Mt provocó un espaciamiento basal de alrededor 1,5 $\mathrm{nm}$ en ambas muestras, correspondiente a un arreglo del TBZ paralelo a las láminas siloxánicas, de acuerdo a lo descripto por Lombardi et al. [2003; 2006]. El aumento en el espaciado basal en las montmorillonitas naturales confirma que los sitios de carga permanente están involucrados en la adsorción del fungicida en estos materiales.

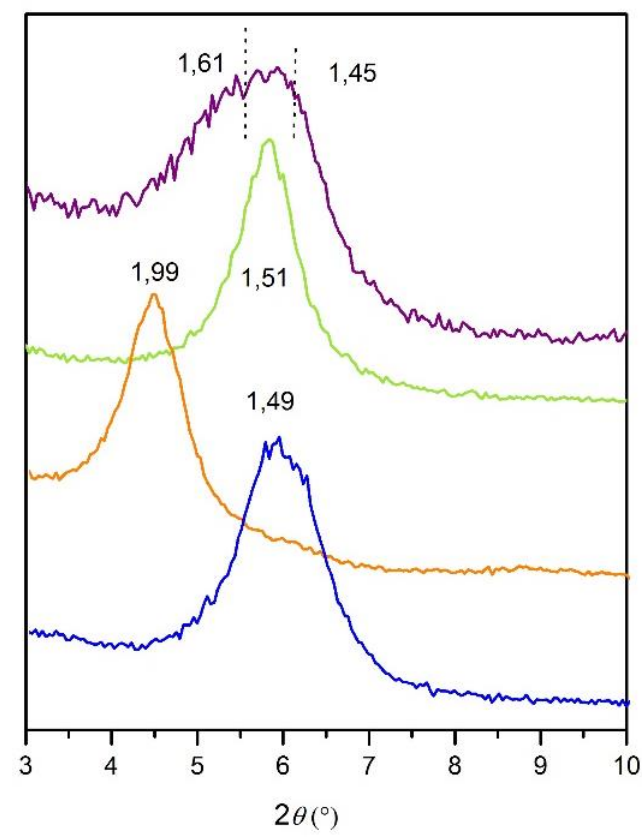

Figura 4.8 Difractogramas de los productos de adsorción y espaciado basal (en nm) de $(-)$ TBZ-Mt, $(-)$ TBZ-OMt55, (一) TBZ-OMt137, (-) TBZ-Wy y (-) TBZ-OWy. 
Por otro lado, la adsorción de TBZ no produjo un incremento del espaciado basal en las organo-montmorillonitas. La muestra TBZ-OWy presentó un pico $\mathrm{d}_{001}$ correspondiente a 1,99 nm -igual que el adsorbente inicial (Figura 4.2)- lo cual es coherente con la idea de que el TBZ no ingresa a los sitios interlaminares y que son otros sitios activos a los cuales se une. La deconvolución del pico $\mathrm{d}_{001}$ de TBZ-OMt55 condujo a dos componentes correspondientes a espaciados basales de 1,61 y 1,45 nm, similares a aquellos encontrados para OMt55 (Figura 3.2). En este caso, frente a los datos de desorción encontrados que evidenciaron fuertes interacciones entre el $\mathrm{TBZH}^{+}$y la superficie negativa del sólido, se propone que en este caso, las láminas están lo suficientemente separadas para que el TBZ penetre en las galerías interlaminares, sin generar un aumento del espaciado.

La Tabla 4.5 presentan los datos de Tg, DTg y ATD de los productos de adsorción. A pesar de que la adsorción del fungicida no generó picos tan claramente resueltos como en el caso de la adsorción de IMZ, analizado en el Capítulo 3 -probablemente debido a la baja cantidad adsorbida de TBZ-, se observaron cambios en las temperaturas de los picos DTg y ATD así como también cambios en los porcentajes de pérdida de masa en las curvas Tg que permitieron inferir la adsorción del mismo y que se analizan a continuación.

La adsorción de TBZ en las montmorillonitas naturales (Wy y Mt) provocó dos efectos en la Región I (Figura 4.9, Tabla 4.5): por un lado disminuyó la temperatura del primer proceso de deshidratación (desorción de agua fisisorbida) dando cuenta de la presencia de TBZ en la superficie externa de las muestras. Por otro lado, aparecieron nuevos picos ATD y DTg asociados a la deshidratación de agua unida a cationes interlaminares. Este fenómeno pudo deberse a cambios en el agua interlaminar, por ejemplo, la formación de uniones hidrógeno entre $\mathrm{TBZH}^{+}$y moléculas de agua unidas a los cationes inorgánicos remanentes en la intercapa [Wicklein et al., 2010].

En la Región II se registraron en Tg incrementos en la pérdida de masa de 0,3\% a 1,3\% en Wy y TBZ-Wy, respectivamente, y de $0,5 \%$ a $1,2 \%$ en Mt y TBZ-Mt, respectivamente (Tabla 4.5). Además, en esta última muestra pudo detectarse un pico DTg a $337{ }^{\circ} \mathrm{C}$ asociado a un pico exotérmico en el ATD a $322{ }^{\circ} \mathrm{C}$ (Figura 4.9). El TBZ se descompone entre 200 y $305{ }^{\circ} \mathrm{C}$, con un pico DTg centrado a $297{ }^{\circ} \mathrm{C}$ (Figura 4.9). Por lo tanto, el incremento en la pérdida de masa en la Región II, así como los nuevos picos DTg y ATD que aparecieron, se asociaron a la oxidación de TBZ unido a las capas siloxánicas de las montmorillonitas [Maltas y Ozmen, 2015]. La ausencia de estos picos, aun de baja intensidad en la muestra TBZ-Wy, se explicaron por la baja cantidad adsorbida de TBZ en esta muestra. 
Tabla 4.5 Reacciones térmicas, picos DTg y ATD $\left[{ }^{\circ} \mathrm{C}\right]$ y porcentajes de pérdida de masa [\%] de productos de adsorción de TBZ.

\begin{tabular}{|c|c|c|c|c|c|c|}
\hline & & & TBZ-Wy & TBZ-OWy & TBZ-Mt & TBZ-OMt55 \\
\hline \multirow{6}{*}{$\begin{array}{l}\text { Región } \\
\text { I }\end{array}$} & \multicolumn{2}{|c|}{ Intervalo de $\mathrm{T}$} & $30-194$ & $30-107$ & $30-156$ & $30-171$ \\
\hline & \multirow{2}{*}{$\begin{array}{l}\text { Desorción de agua } \\
\text { fisisorbida }\end{array}$} & DTg & 76 & 57 & 53 & 41 \\
\hline & & ATD (endo) & 80 & 67 & 71 & n.d. \\
\hline & \multirow{2}{*}{$\begin{array}{l}\text { Deshidratación de } \\
\text { cationes interlaminares }\end{array}$} & DTg & 141 & n.d. & $116 ; 119 ; 134$ & n.d. \\
\hline & & ATD (endo) & 155 & n.d. & 142 & n.d. \\
\hline & Pérdida de masa total & $\mathrm{Tg}$ & 13,7 & 2,3 & 9,0 & 4,0 \\
\hline \multirow{7}{*}{$\begin{array}{l}\text { Región } \\
\text { II }\end{array}$} & \multicolumn{2}{|c|}{ Intervalo de $\mathrm{T}$} & $194-565$ & $151-383$ & $200-392$ & $211-390$ \\
\hline & \multirow{2}{*}{$\begin{array}{l}\text { 1er Paso de oxidación } \\
\text { de contenido orgánico. }\end{array}$} & DTg & n.d. & $260 ; 355(h)$ & 337 & 304 \\
\hline & & ATD (exo) & n.d. & $284 ; 351(\mathrm{~h})$ & 322 & 307 \\
\hline & \multicolumn{2}{|c|}{ Intervalo de $\mathrm{T}$} & n.d. & $383-492$ & n.d. & $390-487$ \\
\hline & \multirow{2}{*}{$\begin{array}{l}\text { 2do Paso de oxidación } \\
\text { de contenido orgánico. }\end{array}$} & DTg & n.d. & n.d. & n.d. & 440 \\
\hline & & ATD (exo) & n.d. & n.d. & n.d. & n.d. \\
\hline & Pérdida de masa total & $\mathrm{Tg}$ & 1,3 & 25,0 & 1,2 & 8,5 \\
\hline \multirow{6}{*}{$\begin{array}{l}\text { Región } \\
\text { III }\end{array}$} & \multicolumn{2}{|c|}{ Intervalo de $\mathrm{T}$} & $565-749$ & $492-874$ & $556-740$ & $487-831$ \\
\hline & \multirow[t]{2}{*}{ Deshidroxilación } & DTg & 664 & 580 & 659 & 603 \\
\hline & & ATD (endo) & 651 & 591 & 657 & 604 \\
\hline & \multirow{2}{*}{$\begin{array}{l}\text { Oxidación de restos } \\
\text { carbónicos }\end{array}$} & DTg & n.d. & 694 & n.d. & n.d. \\
\hline & & ATD (exo) & n.d. & 698 & n.d. & n.d. \\
\hline & Pérdida de masa total & $\mathrm{Tg}$ & 4,1 & 12,0 & 3,6 & 11,2 \\
\hline
\end{tabular}

n.d.: no determinado; (h): hombro
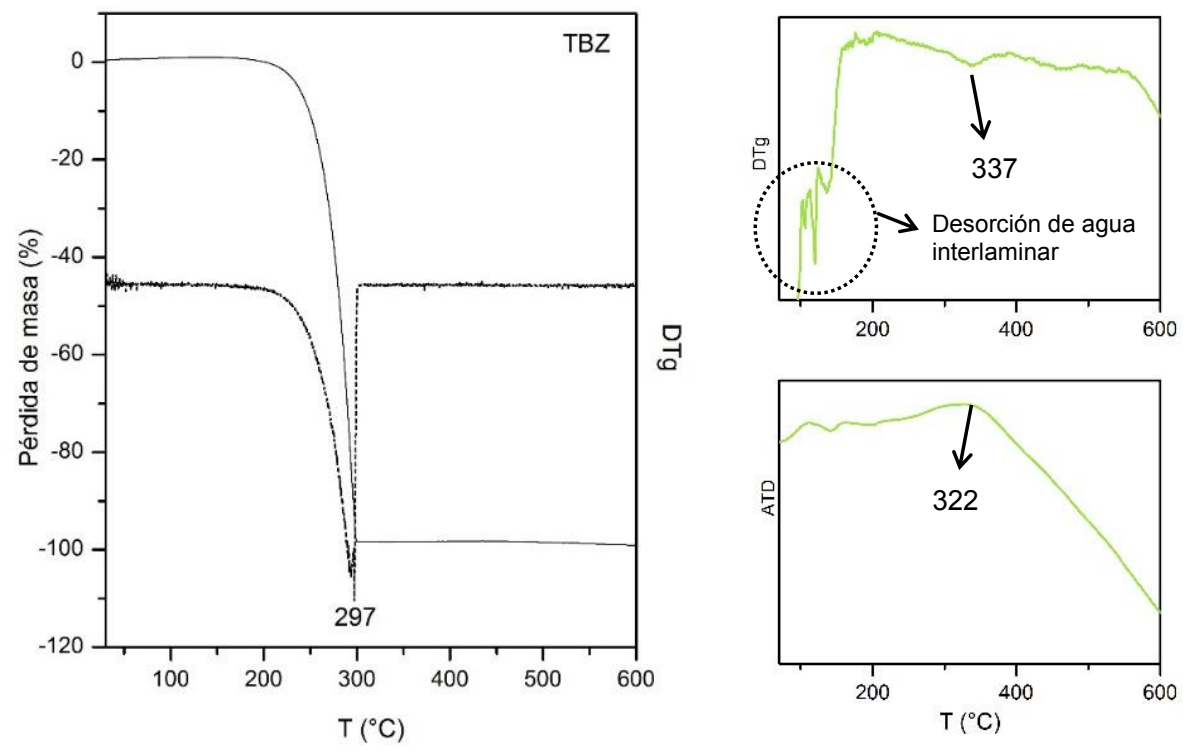

Figura 4.9 Curvas de análisis térmicos de $(-)$ TBZ y $(-)$ TBZ-Mt.

La presencia de TBZ en OWy y OMt55 condujo a la disminución de temperatura de los picos DTg y ATD del primer paso de deshidratación (Región I) evidenciando la mayor 
hidrofobicidad de estas muestras respecto a los materiales de partida por la unión del TBZ en la superficie externa. En OWy este efecto fue mayor ya que estuvo acompañado de la disminución en el porcentaje de pérdida de masa en esta Región (Tabla 4.5).

Es llamativo que en la Región II la adsorción de TBZ generó una disminución del porcentaje de pérdida de masa de 28 a 25\% en OWy y TBZ-OWy, respectivamente. Este fenómeno, producido durante la adsorción de un compuesto orgánico, sólo pudo explicarse por la desorción de moléculas de surfactante unidas como pares iónicos a través de fuerzas de van der Waals. En TBZ-OMt55, en cambio, el porcentaje de pérdida de masa aumentó en esta región respecto a OMt55, nuevamente indicando que las muestras OWy y OMt55 presentan mecanismos y sitios de adsorción de TBZ diferentes.

Tabla 4.6 Composición elemental de la superficie externa determinada por XPS (fracciones molares, \%).

\begin{tabular}{|c|c|c|}
\hline & TBZ & TBZ-OWy \\
\hline $\mathrm{C} 1 s(285 \mathrm{eV})$ & 47,3 & 41,8 \\
$\mathrm{C} 1 s(286,6 \mathrm{eV})$ & 25,0 & 7,9 \\
$\mathrm{C} 1 s(289 \mathrm{eV})$ & 2,6 & 2,5 \\
$\mathrm{C}_{\text {TотAL }}$ & 74,1 & 52,2 \\
$\mathrm{Br} 3 d$ & - & 0,2 \\
$\mathrm{Br} 3 d / \mathrm{Si} 2 p$ & - & 0,020 \\
$\mathrm{~N} 1 s(399 \mathrm{eV})$ & 11,8 & 0,5 \\
$\mathrm{~N} 1 s(402 \mathrm{eV})$ & - & 1,2 \\
$\mathrm{~N} 1 s(402 \mathrm{eV}) /(399 \mathrm{eV})$ & - & 2,4 \\
$\mathrm{~N}_{\text {TOTAL }}$ & 11,8 & 1,7 \\
$\mathrm{Si} 2 p$ & 0,6 & 10,0 \\
$\mathrm{Al} 2 p$ & - & 3,7 \\
$\mathrm{~N}_{1} 1 s$ & 1 & - \\
$\mathrm{Mg}_{\mathrm{KLLL}}$ & - & 1,5 \\
$\mathrm{O}_{\text {TотAL }}$ & 7,5 & 31,8 \\
$\mathrm{~S} 2 p$ & 5 & - \\
\hline
\end{tabular}

La adsorción de TBZ en la superficie de Wy condujo a un incremento de las fracciones molares de los picos correspondientes a C1s en la muestra TBZ-Wy respecto a Wy; 
mientras que el porcentaje de $\mathrm{C}_{\text {TOTAL }}$ disminuyó en TBZ-OWy respecto a OWy (Tablas 4.2 y 4.6). El cociente $\mathrm{Br} 3 d / \mathrm{Si} 2 p$, que para $\mathrm{OWy}$ presentó un valor de 0,076 , disminuyó a 0,020 luego de la adsorción de TBZ. Este comportamiento sugirió que hubo un desplazamiento de ODTMA-Br débilmente unidos cuando ocurre la adsorción del fungicida y apoya los resultados obtenidos por $\mathrm{Tg}$.

Además, la muestra TBZ-OWy, presentó una disminución del 40\% del cociente N1s (402)/(399) respecto a OWy. Esto, nuevamente sugiere un desplazamiento de moléculas ODTMA débilmente unidas en la superficie externa debido a la adsorción de TBZ (Tablas 4.2 y 4.6).

Las medidas de potencial zeta (Tabla 4.7) permitieron inferir que en las montmorillonitas naturales (Wy y Mt) los sitios de superficie externa también son sitios activos en la adsorción del fungicida, ya que en ambos casos los productos de adsorción presentaron valores de potencial zeta menos negativos que las muestras iniciales (Tabla 4.7, Figuras 3.3), indicando que la forma protonada del fungicida se une en las caras externas de las muestras. La muestra TBZ-OWy presentó, por el contrario, una disminución en la carga positiva desde $+40 \mathrm{a}+26 \mathrm{mV}$, que es coherente con la pérdida de cationes ODTMA ${ }^{+}$ durante la adsorción del fungicida. La carga eléctrica superficial de la muestra OMt55 no se modificó con la adsorción del fungicida (Tabla 4.6, Figuras 3.4 y 4.4) lo cual sugiere que no son estos sitios a los que se une el fungicida.

Tabla 4.7 Potencial zeta $(\mathrm{pH}=7)$ de productos de adsorción de TBZ $\left(C_{i}=25 \mathrm{mg} / \mathrm{L}\right)$.

\begin{tabular}{|c|c|}
\hline Muestra & Potencial zeta $[\mathrm{mV}]$ \\
\hline TBZ-Mt & $-18 \pm 1$ \\
TBZ-OMt55 & $-30,1 \pm 0,9$ \\
TBZ-Wy & $-19,8 \pm 0,5$ \\
TBZ-OWy & $26 \pm 1$ \\
\hline
\end{tabular}

El arreglo de TBZ como molécula neutra (TBZ) o como cation $\left(\mathrm{TBZH}^{+}\right)$en la superficie externa se investigó para Wy (los modelos se llamaron Wy-TBZ y Wy-TBZH ${ }^{+}$) y para OWy $\left(\mathrm{OWy}-\mathrm{TBZ}\right.$ y OWy- $\left.\mathrm{TBZH}^{+}\right)$.

Los sistemas donde se comparó el arreglo de la molécula en su forma neutra y en su forma catiónica, se investigaron comenzando con la misma configuración inicial de TBZ y $\mathrm{TBZH}^{+}$. Se calculó un valor promedio de energía de interacción entre las dos formas del 
fungicida y la superficie de Wy u OWy, empleando para ello las últimas 30 configuraciones de la simulación. El cálculo de la interacción entre el adsorbato y la superficie del adsorbente $\left(E_{i n t}\right)$ se determinó de la siguiente manera:

$$
E_{\text {int }}=E_{\text {total }}-E_{\text {superficie }}-E_{\mathrm{H}_{2} \mathrm{O}}-E_{\left(\text {superficie }+\mathrm{H}_{2} \mathrm{O}\right)}-E_{\left(\mathrm{TBZ}+\mathrm{H}_{2} \mathrm{O}\right)}-E_{\mathrm{TBZ}}
$$

(Ecuación 4.3)

donde $E_{\text {total }}$ es la energía potencial total del sistema; $E_{\text {superficie }}, E_{H 2 O}, E_{\text {(superficie }+H 2 O)}$ son las energías potenciales de las superficies de Wy u OWy, de las moléculas de agua (y su interacción mutua) y de la interacción de Wy con las moléculas de agua, respectivamente; $E\left({ }_{T B Z+H 2 O}\right)$ es la energía de interacción entre TBZ y moléculas de agua; y $E_{T B Z}$ es la energía potencial de TBZ.

En el caso de $\mathrm{Wy}_{\mathrm{y}}-\mathrm{TBZH}^{+}$, se obtuvieron interacciones electrostáticas de $-243 \mathrm{kcal} / \mathrm{mol}$ entre las láminas negativas y el catión $\mathrm{TBZH}^{+}$, llevando a un arreglo paralelo de la molécula respecto de la superficie (Figura 4.10). La energía de interacción repulsiva entre $\mathrm{TBZH}^{+}$y $\mathrm{Na}^{+}$fue de $+23 \mathrm{kcal} / \mathrm{mol}$. El TBZ neutro en el modelo Wy-TBZ exhibió diferente comportamiento en la superficie $\mathrm{Wy}$ debido a que la interacción atractiva entre la molécula y la superficie fue mucho más débil $(-10 \mathrm{kcal} / \mathrm{mol})$. Las interacciones dominantes fueron, en cambio, entre la molécula TBZ y el catión $\mathrm{Na}^{+}$, que formaron un complejo con $E_{\text {int }}=$ $41 \mathrm{kcal} / \mathrm{mol}$ (Figura 4.10). El Na${ }^{+}$se localizó entre los dos átomos de nitrógeno de la molécula de TBZ. El fungicida en el complejo se orientó de forma paralela o levemente inclinada respecto a la superficie siloxánica. Como durante la adsorción del TBZ se obtuvo un incremento en el $\mathrm{pH}$ y no se observó desorción, se propone que el modelo $\mathrm{Wy}_{\mathrm{y}} \mathrm{TBZH}^{+}$ es más realista, mientras que la formación de complejo es un caso hipotético.
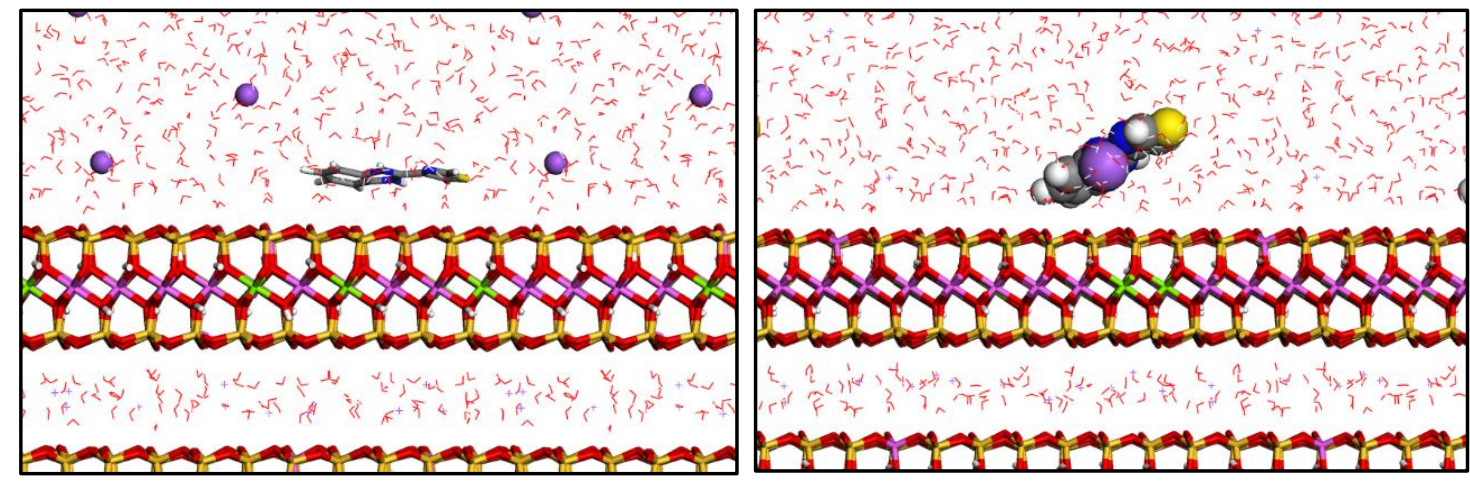

Figure 4.10 Izquierda: arreglo de $\mathrm{TBZH}^{+}$en la superficie de Wy. Derecha: arreglo del complejo TBZ-Na ${ }^{+}$en la superficie de Wy. 
En base a los resultados tanto de potencial zeta como de XPS, la caja de simulación de OWy se creó situando seis cationes ODTMA que compensaron la carga de capa y se orientaron en la superficie con sus átomos de nitrógeno cercanos a las cavidades hexagonales de la capa tetraédrica. Además, se ubicaron tres pares iónicos ODTMA-Br. Los cationes ODTMA unidos electrostáticamente, así como los pares iónicos ODTMA-Br, formaron un arreglo bilaminar desordenado en la superficie externa. Las cadenas alifáticas presentaron una orientación inclinada respecto de las láminas (Figura 4.11). Asimismo, en la dinámica se observó que los iones $\mathrm{Br}^{-}$se disociaban y quedaban disueltos en la fase acuosa. En total los nueve cationes ODTMA en la superficie externa presentaron una interacción repulsiva de $+840 \mathrm{kcal} / \mathrm{mol}$.
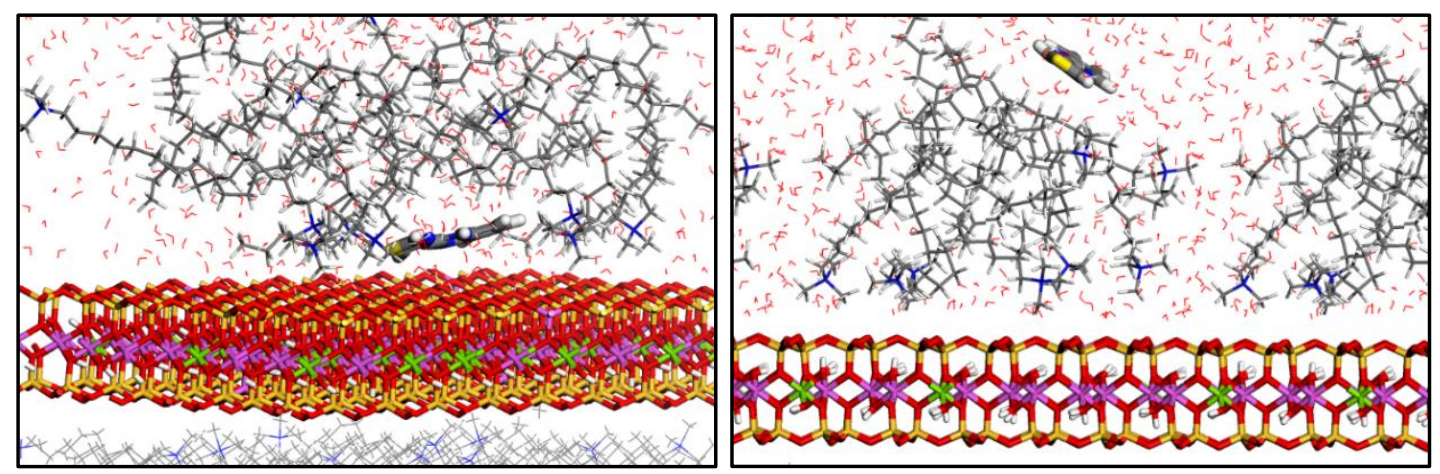

Figura 4.11 Izquierda: $\mathrm{TBZH}^{+}$unido a los sitios de superficie externa, ubicados debajo de los cationes ODTMA en el modelo OWy-TBZH ${ }^{+}$. Derecha: TBZ neutro situado en los sitios hidrofóbicos en el modelo OWy-TBZ.

Para estudiar el arreglo de TBZ en OWy, el TBZ se colocó entre las cadenas de los surfactantes en los modelos iniciales. El catión $\mathrm{TBZH}^{+}$se vio atraído por los sitios de superficie siloxánica y adoptó un arreglo paralelo o inclinado respecto de ella. Más aún, el catión $\mathrm{TBZH}^{+}$tendió a ubicarse por debajo de algunos cationes ODTMA y expulsarlos de la misma (Figura 4.11). Esto puede explicarse comparando las energías de interacción entre los cationes ODTMA y el catión $\mathrm{TBZH}^{+}$y la superficie siloxánica. La energía de interacción ODTMA-superficie siloxánica fue de $-44 \mathrm{kcal} / \mathrm{mol}$, mientras que la de $\mathrm{TBZH}^{+}$superficie siloxánica fue de $-190 \mathrm{kcal} / \mathrm{mol}$ (cercanas a las de $\mathrm{Wy}_{\mathrm{y}}-\mathrm{TBZH}^{+}$). Esta gran diferencia de energías de interacción condujo a una preferencia de $\mathrm{TBZH}^{+}$respecto de ODTMA en los sitios de superficie externa. Estos resultados están de acuerdo con los datos experimentales (XPS, Tg, potencial zeta) donde se observó una disminución de cationes ODTMA en la superficie externa luego de la adsorción de TBZ. Cabe destacar que $\mathrm{TBZH}^{+}$exhibió interacciones repulsivas con las aminas que fueron en valores absolutos, comparables con las interacciones atractivas $\mathrm{TBZH}^{+}$- superficie siloxánica. Esto indica que 
los cationes ODTMA pueden parcialmente evitar que el catión $\mathrm{TBZH}^{+}$interactúe con los sitios de la superficie externa, lo que explicaría los bajos valores de adsorción y altos valores de desorción encontrados experimentalmente. El TBZ en forma neutra interactuó principalmente mediante interacciones van der Waals con los sitios hidrofóbicos, es decir en la fase orgánica formada por las cadenas alquílicas a través del mecanismo de partición organofílica (Figura 4.10). La energía de interacción entre la molécula neutra TBZ y los cationes ODTMA o la superficie siloxánica fue $-7 \mathrm{kcal} / \mathrm{mol}$ en promedio.

\subsection{Conclusiones parciales}

En este capítulo se estudió la adsorción del fungicida tiabendazol en montmorillonitas de origen nacional e internacional y en organo-montmorillonitas obtenidas a partir de éstas, por intercambio catiónico con el surfactante catiónico bromuro de octadeciltrimetilamonio.

En base a los datos experimentales, y de acuerdo a lo indicado en bibliografía, se concluyó que el mecanismo que explica la adsorción de TBZ en las montmorillonitas naturales y en la OMt con ODTMA al $55 \% \mathrm{CIC}$, es el intercambio catiónico entre $\mathrm{TBZH}^{+}$ y los cationes $\mathrm{Na}^{+}$interlaminares, similar a lo encontrado para el fungicida IMZ, también de naturaleza básica en el Capítulo 3 de la presente tesis. Los sitios activos involucrados fueron principalmente los sitios de carga permanente. La presencia de ODTMA disminuyó la cantidad adsorbida del fungicida debido a que la amina cuaternaria y el $\mathrm{TBZH}^{+}$se unen a los mismos sitios activos en la montmorillonita. Estas muestras no presentaron desorción, inclusive después de tres días, en las condiciones estudiadas, debido a la fuerte interacción electrostática entre el adsorbato y el adsorbente que pudo ser calculada por dinámica molecular.

Las OMts con porcentaje de ODTMA superior al $100 \% \mathrm{CIC}$ mostraron que el mecanismo y los sitios de adsorción, así como las fuerzas de interacción entre el adsorbato y el adsorbente, son de otra naturaleza. En estas muestras el bloqueo de los sitios de carga permanente por parte de las aminas cuaternarias disminuye la capacidad adsorbente de las mismas. Las simulaciones computacionales permitieron evidenciar que el fungicida en su forma protonada es capaz de unirse a la superficie siloxánica externa y expulsar a cationes ODTMA. Las fuerzas motoras de esta reacción son la energía de repulsión electrostática, la cual es muy alta entre los cationes ODTMA en la superficie externa de estas muestras (y no así en otras con menor contenido de ODTMA, como OMt55, analizada anteriormente) y la energía de interacción electrostática atractiva entre el $\mathrm{TBZH}^{+}$y la lámina siloxánica negativa, que supera en magnitud a la energía de interacción entre dicha lámina y el cation 
$\mathrm{ODTMA}^{+}$. Este mecanismo -que finalmente es un intercambio catiónico entre el $\mathrm{TBZH}^{+} \mathrm{y}$ los cationes ODTMA ${ }^{+}$unidos a los sitios de superficie externa- explicarían la disminución de contenido orgánico observado en Tg, los cambios de composición química evidenciado por XPS, la disminución de carga eléctrica positiva obtenido por potencial zeta y la baja capacidad de adsorción de estas muestras, ya que la energías electrostáticas de repulsión entre el $\mathrm{TBZH}^{+}$y $\mathrm{ODTMA}^{+}$en la superficie externa, evitarían parcialmente la adsorción del compuesto. El 82\% de desorción obtenido para TBZ en las organo-montmorillonitas con ODTMA mayor al $100 \%$ CIC, podría deberse a dos comportamientos: el complejo formado es inestable por las fuerzas repulsivas intervinientes y por lo tanto desorbe $\mathrm{TBZH}^{+}$, o el TBZ se une en forma neutra a los sitios hidrofóbicos por mecanismo de partición organofílica en la fase orgánica formada por las cadenas alquílicas del surfactante a través de interacciones de van der Waals, débiles. 


\section{Capitulo 5. \\ Adsorción de imazalily tiabendazol en montmorillonitas intercambiadas con $\mathrm{Cu}^{2+}$.}

\subsection{Introducción y objetivos específicos}

Los Capítulos 3 y 4 mostraron que la incorporación de cadenas alquílicas en la estructura de montmorillonitas no generó nuevos sitios activos en la adsorción de TBZ e IMZ. En cambio, actuaron disminuyendo la cantidad de sitios activos disponibles para la unión de los fungicidas, efecto que fue más importante a altas cantidades de amina en el sólido.

En ese sentido, la hipótesis planteada -donde el agregado de una fase orgánica en el adsorbente mejoraría la adsorción de compuestos orgánicos- no se cumplió. En consecuencia, para mejorar la adsorción, se recurrió a otra estrategia.

Las simulaciones computacionales del sistema Wy-TBZ mostró que la molécula neutra tendía a coordinarse con el catión interlaminar inorgánico $\left(\mathrm{Na}^{+}\right)$. Aunque ese modelo fue descartado por no ajustarse a los datos experimentales, dio la pauta de que teóricamente ese proceso podría ocurrir. Más aún, muchos trabajos reportan la síntesis y caracterización de complejos de coordinación estables, donde el TBZ actúa como ligando bidentado, y el centro metálico corresponde a cationes metálicos alcalinos, alcalinotérreos y de transición [Kowala et al., 1971; Grevy et al., 2002; Sethuraman et al., 2002; Mothilal et al., 2003; Sączewski et al., 2006; Devereux et al., 2007; Xu et al., 2014]. Todos ellos son concluyentes en cuanto a que, tanto en solución como en estado sólido, el TBZ se coordina a los 
cationes metálicos a través de los átomos de nitrógeno de los anillos imidazol y tiazol (tal como se vio por DM en el Capítulo 4), independientemente de la naturaleza del catión metálico [Grevy et al., 2002].

Teniendo en cuenta estos antecedentes y que el imazalil también posee un grupo imidazol, en este capítulo se propuso generar un material donde los sitios activos para la unión de TBZ e IMZ fueran cationes metálicos interlaminares, mediante el intercambio de $\mathrm{Na}^{+}$por el metal de transición $\mathrm{Cu}^{2+}$. Se decidió trabajar con este metal por varias razones. Por un lado, la posibilidad de que la incorporación de un catión "blando" favorezca la unión de aminas aromáticas (según la teoría ABDB, Sección 2.2.4). Por otro lado, el $\mathrm{Cu}^{2+}$ está presente en aguas de escorrentía provenientes de las actividades minera e industrial. El Programa Internacional de Seguridad de las Sustancias Químicas de la Organización Mundial de la Salud establece que el límite máximo aceptable de ingesta oral diaria en personas adultas, aunque incierto, es probable que esté en el rango de los mg diarios [OMS, 2011] por lo que su remoción de efluentes es necesaria. Por lo tanto, la estrategia delineada permitiría utilizar la montmorillonita primero en la remoción de $\mathrm{Cu}^{2+}$ de efluentes mineros e industriales y luego en la remoción de fungicidas de efluentes agrícolas. Además, la utilización de montmorillonitas para la adsorción de $\mathrm{Cu}^{2+}$ ha sido ampliamente reportada [Madejová et al., 1999; Hyun et al., 2000; He et. al., 2001; Morton et al., 2001; Ding y Frost, 2004; Al-Qunaibit et al., 2005; Madejová et al., 2006; Brtáňová et al., 2014; Glatstein y Francisca, 2015; Zhu et al., 2015] y se conoce fehacientemente que el metal se une a los sitios de carga permanente y de superficie externa, desplazando a los cationes monovalentes y, dependiendo del $\mathrm{pH}$ de la reacción, también en los sitios de borde ${ }^{7}$. Este amplio bagaje de conocimientos sobre montmorillonitas intercambiadas con $\mathrm{Cu}^{2+}$ constituye un motivo más por el cual se eligió este metal para evaluar la adsorción de los fungicidas.

También en este capítulo se encaró el estudio de la influencia de la presencia de un segundo fungicida en la adsorción del otro. Para ello se estudió la adsorción de ambos fungicidas en un mismo adsorbente, tanto en forma simultánea como secuencial. A las isotermas de adsorción y caracterizaciones de adsorbente y productos se sumaron cinéticas de adsorción de los fungicidas por separado para dilucidar las afinidades relativas del

\footnotetext{
${ }^{7}$ Además, hay una discusión en la bibliografía acerca de la ocupación por parte de $\mathrm{Cu}^{2+}$ de las cavidades hexagonales de la capa siloxánica y de su penetración a las vacancias octaédricas [Madejová et al., 1999, 2006; He et al., 2001; Zhu et al., 2015].
} 
adsorbente por los adsorbatos y evaluar la existencia de competencias por los sitios de adsorción o efectos sinérgicos. Así, los objetivos específicos de este capítulo fueron:

(i) obtener una montmorillonita intercambiada con $\mathrm{Cu}^{2+}$;

(ii) estudiar su capacidad de remoción de tiabendazol e imazalil, tanto en sistemas individuales como en mezclas, determinar las leyes de velocidad que rigen la adsorción de los fungicidas por separado, evaluar afinidades relativas, sinergias y antagonismos en la adsorción de los fungicidas en simultáneo;

(iii) caracterizar el material de partida y los productos de adsorción, y determinar sitios de adsorción y mecanismos involucrados;

(iv) comparar su desempeño respecto a la muestra natural.

\subsection{Materiales y métodos}

\subsubsection{Materiales}

En este capítulo se prepararon soluciones madre (acuosas) de tiabendazol ( $25 \mathrm{mg} / \mathrm{L}$ ) e imazalil $(150 \mathrm{mg} / \mathrm{L})$ grado estándar provistos por Sigma-Aldrich. Las soluciones de concentración más bajas se prepararon mediante diluciones de éstas en agua destilada.

Se preparó una Mt intercambiada con $\mathrm{Cu}^{2+}$ (denominada $\mathrm{Mt}-\mathrm{Cu}$ ) agregando lentamente y bajo agitación constante $10 \mathrm{~g}$ de Mt (proporcionada por Castiglioni Pes y Cia, Sección 3.2.1) a $500 \mathrm{~mL}$ de una solución $\mathrm{CuSO}_{4} \cdot 5 \mathrm{H}_{2} \mathrm{O}$ 0,1 $\mathrm{M}$ y dejando en agitación durante toda la noche. El pH de la suspensión se mantuvo constante en 4,8. El producto se centrifugó y lavó con agua destilada hasta lograr la remoción del exceso de $\mathrm{Cu}^{2+}$ (determinado por espectrofotometría mediante la prueba del Dietilditiocarbamato de sodio [Kolthoff y Sandell, 1943]). El sólido obtenido se secó en estufa a $80{ }^{\circ} \mathrm{C}$ durante 72 h, se molió en mortero de ágata y se mantuvo en desecador para su caracterización y/o utilización posterior.

El contenido de $\mathrm{Cu}^{2+}$ en el sólido fue de 0,309 mmol/g equivalente a 74,9\% \% teniendo en cuenta la doble carga del $\mathrm{Cu}^{2+}$. Para determinarlo, $1 \mathrm{~g}$ de muestra fue digerida con $20 \mathrm{~mL}$ de $\mathrm{HNO}_{3}$ concentrado a $60{ }^{\circ} \mathrm{C}$ hasta la evaporación del líquido. Este procedimiento se repitió, luego el sólido fue resuspendido en una solución de $\mathrm{HNO}_{3}: \mathrm{H}_{2} \mathrm{O}$ (1:1) y la suspensión fue filtrada para recuperar el sobrenadante. La concentración de $\mathrm{Cu}^{2+}$ en el sobrenadante se determinó por absorción atómica usando un equipo SensAA dual GBC Sci. 


\subsubsection{Estudios de adsorción}

\subsubsection{Cinéticas de adsorción}

Los estudios cinéticos comprenden el estudio de la velocidad de los procesos químicos y de los factores que influyen en ésta. La determinación de la ley de velocidad que rige un proceso de adsorción proporciona información sobre el mecanismo y los diferentes estados de transición en el camino hacia la formación del complejo final adsorbato-adsorbente [Gupta y Bhattacharyya, 2011].

La velocidad de una reacción depende de la concentración de las especies involucradas en el proceso. La ley de velocidad en general obedece a la forma

$$
R=k[A]^{a}[B]^{b}
$$

(Ecuación 5.1)

donde $k$ es la constante de la velocidad y los coeficiente $a, b$, etc. representan el orden respecto a la concentración de las especies A, B, etc. La forma exacta de la ley de velocidad se determina experimentalmente, construyendo curvas de cantidad adsorbida por unidad de masa $\left(q_{t}\right)$ en función del tiempo $(t)$ y luego ajustando los datos a diferentes modelos.

En este trabajo se realizaron cinéticas de adsorción de los fungicidas individuales en la muestra Mt-Cu. Las cinéticas se realizaron en sistema batch a $25{ }^{\circ} \mathrm{C}$ en tubos de plástico empleando tiempos de contacto de $10 \mathrm{~min}$ a $72 \mathrm{~h}$. Una vez transcurrido el tiempo correspondiente se centrifugaron las suspensiones a $14.000 \mathrm{rpm}$ durante $15 \mathrm{~min}$. La concentración de los fungicidas en los sobrenadantes se determinó mediante cromatografía líquida de alta resolución (HPLC) en las condiciones descriptas en las Secciones 3.2.3 y 4.2.3.

Tabla 5.1 Condiciones y nomenclatura de las cinéticas de adsorción de TBZ e IMZ en Mt-Cu.

\begin{tabular}{|c|c|c|c|}
\hline Adsorbato & $C_{i}[\mathrm{mg} / \mathrm{L}]$ & Relación sólido/solución $[\mathrm{g} / \mathrm{L}]$ & Denominación \\
\hline TBZ & 15 & 1 & TBZ-1 \\
& 20 & 0,1 & TBZ-0,1 \\
\hline IMZ & 40 & 1 & IMZ-1 \\
& 100 & 0,1 & IMZ-0,1 \\
\hline
\end{tabular}

Las cinéticas se realizaron en distintas condiciones de concentración inicial y relación sólido/solución. Para facilitar la presentación de los resultados a cada curva $q_{t}$ vs. $t$ se la identificó con una nomenclatura (Tabla 5.1). En una primera instancia se trabajó con la 
relación sólido/solución $=1 \mathrm{~g} / \mathrm{L}$ y concentraciones iniciales de los fungicidas de: $C_{i}(\mathrm{TBZ})$ $=15 \mathrm{mg} / \mathrm{L}$ (cinética TBZ-1) y $C_{i}(\mathrm{IMZ})=40 \mathrm{mg} / \mathrm{L}$ (cinética IMZ-1). Debido a que con esta relación sólido/solución la cantidad adsorbida fue muy alta (y consecuentemente la concentración final de los fungicidas en la solución cayeron por debajo de los límites de cuantificación, lo cual otorgaría errores muy altos al ajustar los distintos modelos matemáticos a los datos experimentales), se decidió disminuir la relación sólido/solución a $0,1 \mathrm{~g} / \mathrm{L}$ y aumentar las concentraciones iniciales de los fungicidas a $C_{i}(\mathrm{TBZ})=20 \mathrm{mg} / \mathrm{L}$ (cinética TBZ-0,1) y $C_{i}(\mathrm{IMZ})=100 \mathrm{mg} / \mathrm{L}$ (cinética IMZ-0,1). Esta nomenclatura, junto a las condiciones de los ensayos, se muestra en Tabla 5.1.

Los modelos matemáticos que se evaluaron para determinar las leyes de velocidad que rigen en la adsorción de ambos fungicidas se describen a continuación.

(i) Lagergren o Pseudo primer orden (P1O)

Es una de las primeras ecuaciones conocidas para describir cinéticas de adsorción de sistemas sólido-líquido y está representada por la siguiente ecuación:

$$
\frac{\mathrm{d} q_{t}}{\mathrm{~d} t}=k_{1}\left(q_{e}-q_{t}\right)
$$

donde $k_{1}[1 / \mathrm{min}]$ es la constante de velocidad de P1O; $q_{e}$ y $q_{t}$ son las cantidades adsorbidas por unidad de masa en el equilibrio y a cualquier tiempo $t$, respectivamente. La forma integrada de la Ecuación 5.2 (con las condiciones de borde $q_{t}=0$ para $t=0, \mathrm{y} q_{t}=q_{t}$ para $t$ $=t)$ es

$$
\ln \left(q_{e}-q_{t}\right)=\ln q_{e}-k_{1} t
$$

El valor de $k_{1}$ se obtiene de la pendiente de la curva del gráfico $\ln \left(q_{e}-q_{t}\right)$ vs. $t$. Éste depende de la concentración inicial del adsorbato (usualmente, disminuye con el aumento de la concentración inicial) y varía de un sistema a otro.

Es necesario conocer el valor de $q_{e}$ para ajustar la Ecuación 5.3 a los datos experimentales. Esto puede obtenerse de la curva $q_{t}$ vs. $t$-extrapolándolo del valor de $q_{t}$ a tiempos largos- o de isotermas de adsorción realizadas a tiempos suficientemente largos.

Para muchos sistemas se observa que la Ecuación 5.3 describe adecuadamente los datos para los primeros 20 o 30 min de interacción y no para todo el intervalo de tiempo. Sin embargo, si no ajustan todos los puntos experimentales, entonces el mecanismo que sigue el proceso de adsorción es más complejo. Otra forma de validar este modelo es comparando el valor experimental de $q_{e}$ con aquel que se obtiene de la ordenada al origen de la curva $\ln \left(q_{e}-q_{t}\right)$ vs. $t$. 
(ii) Pseudo segundo orden (P2O)

Este modelo puede ser evaluado en términos de la Ecuación 5.4:

$$
\frac{\mathrm{d} q_{t}}{\mathrm{~d} t}=k_{2}\left(q_{e}-q_{t}\right)^{2}
$$

donde $k_{2}$ [g/(mg.min)] es la constante de velocidad de segundo orden. Ésta normalmente depende de las condiciones experimentales como la concentración inicial del adsorbato, el $\mathrm{pH}$ de la suspensión, la temperatura, etc.

La separación de variables, seguido de la integración de la Ecuación 5.4 (con las condiciones de borde $q_{t}=0$ para $t=0, \mathrm{y} q_{t}=q_{t}$ para $t=t$ ) da como resultado la expresión

$$
\frac{t}{q_{t}}=\frac{1}{k_{2} q_{e}^{2}}+\left(\frac{1}{q_{e}}\right) t
$$

donde se observa que la razón $t / q_{t}$ depende linealmente de $t$.

La velocidad inicial $(t \rightarrow 0)$ de adsorción de un proceso que sigue una cinética $\mathrm{P} 2 \mathrm{O}, b$ $[\mathrm{mg} /($ g.min) $]$, se define como

$$
h=k_{2} q_{e}^{2}
$$

(Ecuación 5.6)

Este parámetro, junto con la capacidad de adsorción en el equilibrio $q_{e}$ y la constante de velocidad de segundo orden $k_{2}$ pueden determinarse experimentalmente a partir de la ordenada al origen y la pendiente del gráfico $t / q_{t}$ vs. $t$. Nuevamente, conociendo la capacidad de adsorción en el equilibrio experimental puede compararse con el valor $q_{e}$ teórico que la aplicación de la Ecuación 5.5 devuelve.

(iii) Difusión intra-partícula (DIP)

Para materiales porosos, es necesario tener en cuenta la difusión de las moléculas de adsorbato en los poros como un posible proceso que controle la velocidad de adsorción. La expresión matemática que expresa este modelo cinético es

$$
\frac{q_{t}}{q_{e}}=1-\left(\frac{6}{\pi^{2}}\right) \sum\left(\frac{1}{n^{2}}\right) \exp \left(-n^{2} \pi^{2} D_{c} \frac{t}{r^{2}}\right)
$$

donde $D_{c}$ es la difusividad intra-cristalina; $r$ es el radio de la partícula; $t$ el tiempo de reacción y la sumatoria se efectúa entre $n=1$ y $n=\alpha$.

Acomodando términos y aplicando logaritmo natural, la Ecuación 5.7 puede ser reescrita en la forma simplificada

$$
\ln \left(1-\frac{q_{t}}{q_{e}}\right)=\left(-\frac{\pi^{2} D_{c}}{r^{2}}\right) t+\ln \left(\frac{6}{\pi^{2}}\right)
$$


por lo que el gráfico $\ln \left(1-q_{t} / q_{e}\right)$ vs. $t$ debería ser lineal con una pendiente $k^{\prime}$ ' $1 /$ min] que se conoce como constante de tiempo de difusión, y es inversamente proporcional al cuadrado del radio de la partícula, según la expresión:

$$
k^{\prime}=\frac{\pi^{2} D_{c}}{r^{2}}
$$

Esta ecuación ha sido utilizada por diferentes investigadores [Allen et al., 1997; Cheung et al., 2001; Choy et al., 2004], para determinar si la difusión intra-partícula es la etapa que controla la dinámica de bioadsorción de iones metálicos [Pinzón-Bedoya y Vera Villamizar, 2009].

(iv) Elovich

La ecuación de Elovich, de aplicación general en procesos de quimisorción, supone que la superficie del sólido es energéticamente heterogénea y por ello los sitios activos de adsorción presentan diferentes energías de activación. Se basa en un mecanismo de reacción de segundo orden para un proceso de reacción heterogénea, donde ni la desorción ni interacciones entre las especies adsorbidas pueden sustancialmente afectar la cinética [Gupta y Bhattacharyya, 2006].

La ecuación de Elovich ha sido utilizada en la forma

$$
\frac{\mathrm{d} q_{t}}{\mathrm{~d} t}=\alpha \exp \left(-\beta q_{t}\right)
$$

(Ecuación 5.10)

donde $\alpha$ y $\beta$ son los coeficientes de Elovich. Asumiendo que $\alpha \beta t>>1$ y las condiciones de borde $q_{t}=0$ para $t=0$ y $q_{t}=q_{t}$ para $t=t$, se puede escribir la ecuación de Elovich en su forma lineal según la Ecuación 5.11:

$$
q_{t}=\frac{1}{\beta} \ln (\alpha \beta)+\frac{1}{\beta} \ln t
$$

(Ecuación 5.11)

Se postula que el coeficiente $\alpha$ [mg/(g.min)] representa la velocidad de quimisorción inicial y $\beta[\mathrm{g} / \mathrm{mg}]$ está relacionado con la superficie cubierta y la energía de activación por quimisorción. Los coeficientes de Elovich pueden determinarse del gráfico $q_{t}$ vs. $t$.

Este modelo ha mostrado resultados satisfactorios en la identificación del mecanismo que controla procesos de adsorción de un soluto en fase líquida a partir de un sólido adsorbente [Cheung et al., 2000; Özacar y Şengil, 2005; Pérez-Marín et al., 2007; PinzónBedoya y Vera Villamizar, 2009]. 
Además del valor de $q_{e} \mathrm{y}$ de $\mathrm{R}^{2}$ que se determinan mediante ajustes de los modelos a los datos experimentales, otra forma de evaluar la validez de un modelo cinético es usando la desviación estándar porcentual $(\Delta q[\%])$ que se calcula mediante la siguiente ecuación:

$$
\Delta q[\%]=\sqrt{\frac{\sum\left[\left(q_{t}-q_{t, c a l}\right) / q_{t}\right]^{2}}{n-1}} \times 100
$$

donde $q_{t}$ y $q_{t, a l}$ son los valores de cantidad de fungicida adsorbido por unidad de masa de adsorbente a cada tiempo $t$ experimental y calculado, respectivamente; y $n$ es el número de puntos medidos. El modelo con $\Delta q[\%]$ más bajo es el que se considera que mejor describe el proceso [Saha et al., 2013].

\subsubsection{Isotermas de adsorción}

Se realizaron isotermas de adsorción de los fungicidas individuales y combinados en forma simultánea (Sim) y consecutiva (Con). Para facilitar la presentación de los resultados, las isotermas se identificaron con una nomenclatura, según lo especificado en la Tabla 5.2.

Tabla 5.2 Condiciones y nomenclatura de las isotermas de adsorción.

\begin{tabular}{|c|c|c|c|c|}
\hline Tipo de isoterma & Adsorbato & Adsorbente & $\begin{array}{c}\text { Relación } \\
\text { sólido/solución }[\mathrm{g} / \mathrm{L}]\end{array}$ & Denominación \\
\hline \multirow{8}{*}{$\begin{array}{l}\text { Fungicidas } \\
\text { individuales }\end{array}$} & \multirow[t]{4}{*}{ TBZ } & Mt & 1 & Mt-TBZ-1d \\
\hline & & \multirow{3}{*}{$\mathrm{Mt}-\mathrm{Cu}$} & 1 & Mt-Cu-TBZ-1 \\
\hline & & & 0,5 & Mt-Cu-TBZ-0,5 \\
\hline & & & 0,1 & Mt-Cu-TBZ-0,1 \\
\hline & \multirow[t]{4}{*}{ IMZ } & $\mathrm{Mt}$ & 1 & Mt-IMZ-1 \\
\hline & & & 1 & Mt-Cu-IMZ-1 \\
\hline & & $\mathrm{Mt}-\mathrm{Cu}$ & 0,5 & Mt-Cu-IMZ-0,5 \\
\hline & & & 0,1 & Mt-Cu-IMZ -0,1 \\
\hline \multirow{2}{*}{$\begin{array}{c}\text { Fungicidas } \\
\text { combinados en } \\
\text { forma simultánea }^{\text {a }}\end{array}$} & TBZ & $\mathrm{Mt}-\mathrm{Cu}$ & 0,1 & $\mathrm{Mt}-\mathrm{Cu}-\mathrm{TBZ}(\mathrm{Sim})$ \\
\hline & IMZ & $\mathrm{Mt}-\mathrm{Cu}$ & 0,1 & Mt-Cu-IMZ(Sim) \\
\hline \multirow{2}{*}{$\begin{array}{c}\text { Fungicidas } \\
\text { combinados en } \\
\text { forma consecutiva }\end{array}$} & $\mathrm{TBZ}^{\mathrm{b}}$ & $\mathrm{IMZ}-\mathrm{Mt}-\mathrm{Cu}$ & 0,1 & Mt-Cu-TBZ(Con) \\
\hline & $\mathrm{IMZ}^{\mathrm{c}}$ & TBZ-Mt-Cu & 0,1 & $\mathrm{Mt}-\mathrm{Cu}-\mathrm{IMZ}(\mathrm{Con})$ \\
\hline
\end{tabular}

a: En este ensayo, TBZ e IMZ se encontraban presentes en la misma solución; b: en este ensayo, en una primera instancia se generó el producto de adsorción de IMZ en Mt-Cu (IMZ-Mt-Cu) y luego se determinó la isoterma de adsorción de TBZ en dicho producto; c: en este ensayo, en una primera instancia se generó el producto de adsorción de TBZ en Mt-Cu (TBZ-Mt-Cu) y luego se determinó la isoterma de adsorción de IMZ en dicho producto; d: isoterma presentada en el Capítulo 4. 
(i) Isotermas de adsorción de los fungicidas individuales

Se realizaron isotermas tipo batch de TBZ e IMZ (por separado) en Mt-Cu empleando tres relaciones sólido/solución: 0,1; 0,5 y $1 \mathrm{~g} / \mathrm{L}$. Estas isotermas se compararon con la isoterma de TBZ en Mt a relación sólido/solución $=1 \mathrm{~g} / \mathrm{L}$ que se obtuvo en el Capítulo 4 y la isoterma de IMZ estándar en Mt a la misma relación sólido/solución (Tabla 5.2). En todos los casos, las isotermas se realizaron en tubos de plástico empleando un tiempo de contacto de $24 \mathrm{~h}$ a $25^{\circ} \mathrm{C}$ con agitación constante, en base a los datos cinéticos. El intervalo de concentraciones fue de 2,5 a $150 \mathrm{mg} / \mathrm{L}$ para IMZ y de 0,5 a $25 \mathrm{mg} / \mathrm{L}$ para TBZ. Todas las soluciones fueron preparadas en agua destilada.

Transcurrido el tiempo de contacto, se centrifugaron las suspensiones a $14.000 \mathrm{rpm}$ durante 15 min. Las concentraciones de TBZ e IMZ en los sobrenadantes se determinaron mediante cromatografía líquida de alta resolución (HPLC) acoplado a un detector UVvisible, usando el equipo y las condiciones previamente mencionadas (Sección 3.2.3 y 4.2.3).

El desarrollo de procesos ácido-base durante la adsorción se estudió dejando evolucionar naturalmente el $\mathrm{pH}$ de las suspensiones y midiéndolo al comienzo $(t=0)$ y al final $\left(t_{e q}=24 \mathrm{~h}\right)$. El pH en el punto de máxima concentración de fungicidas en las isotermas Mt-Cu-IMZ-1 $\left(C_{i}(\mathrm{IMZ})=150 \mathrm{mg} / \mathrm{L}\right)$ y Mt-Cu-TBZ-1 $\left(C_{i}(\mathrm{TBZ})=25 \mathrm{mg} / \mathrm{L}\right) \mathrm{se}$ mantuvo constante en 6,1 y 5,6 , respectivamente.

(ii) Isotermas de adsorción de los fungicidas combinados simultáneamente

Las condiciones elegidas para evaluar la adsorción de los fungicidas en simultáneo fueron teniendo en cuenta dos aspectos. Por un lado, el hecho de que para evaluar la "selectividad" del adsorbente respecto a los compuestos, es necesario que las concentraciones sean similares. En ese sentido la solubilidad del TBZ fue la limitante. Por otro lado, el hecho de que al utilizar la relación sólido solución de $1 \mathrm{~g} / \mathrm{L}$, más del $90 \%$ de TBZ e IMZ fueron removidos en las isotermas individuales. Por lo tanto, emplear esa relación sólido/solución afectaría la sensibilidad en la determinación de la concentración final de los fungicidas en el sobrenadante.

Es por ello que las isotermas de adsorción de los fungicidas combinados en simultáneo (isotermas Mt-Cu-TBZ(Sim) y Mt-Cu-IMZ(Sim), Tabla 5.2) se determinaron empleando soluciones equimolares de TBZ e IMZ. Se preparó una solución madre en agua destilada de $C_{i}(\mathrm{TBZ})=C_{i}(\mathrm{IMZ})=0,089 \mathrm{M}$ (equivalente a $18 \mathrm{mg} / \mathrm{L}$ para TBZ y 26,5 mg/L para IMZ). La relación sólido/solución fue de $0,1 \mathrm{~g} / \mathrm{L}$ y el procedimiento seguido fue el mismo que el descripto en (i). 
(iii) Isotermas de adsorción de los fungicidas combinados consecutivamente

Se realizaron isotermas de adsorción de los fungicidas individuales, usando como adsorbente, el producto de la adsorción previa del otro fungicida (productos obtenidos en (i), cuyos datos se muestran en la Tabla 5.3). Es decir, IMZ se adsorbió en el producto TBZ-Mt-Cu y TBZ se adsorbió en IMZ-Mt-Cu. Ambas isotermas, denominadas Mt-CuIMZ(Con) y Mt-Cu-TBZ(Con), respectivamente (Tabla 5.2), se realizaron siguiendo el procedimiento y las condiciones descriptas en (i).

En todas las isotermas, se evaluó el ajuste de los modelos Langmuir, Freundlich y Langmuir-Freundlich (LF) a los datos experimentales.

\subsubsection{Métodos de caracterización}

Se presentan las caracterizaciones del adsorbente $(\mathrm{Mt}-\mathrm{Cu})$ y productos de adsorción de los fungicidas individuales mediante DRX, potencial zeta y análisis térmicos en las condiciones señaladas en el Capítulo 3. La nomenclatura de los sólidos caracterizados, así como la cantidad de fungicida y $\mathrm{Cu}^{2+}$ determinadas en cada uno de ellos, se muestran en la Tabla 5.3.

Las muestras TBZ-Mt-Cu e IMZ-Mt-Cu (Tabla 5.3) se obtuvieron aislando los sólidos con mayor $Q$ en las isotermas Mt-Cu-TBZ-1 y Mt-Cu-IMZ-1, respectivamente, se lavaron con agua destilada, se secaron en estufa a $60^{\circ} \mathrm{C}$ y se conservaron en desecador.

Tabla 5.3 Concentraciones iniciales y cantidades adsorbidas por unidad de masa de fungicidas, y contenido de $\mathrm{Cu}^{2+}$ en los productos de adsorción caracterizados.

\begin{tabular}{|c|c|c|c|c|}
\hline Muestra & $C_{i}[\mathrm{mg} / \mathrm{L}]$ & $\mathcal{Q}[\mathrm{mg} / \mathrm{g}]$ & $\mathcal{Q}[\mathrm{mmol} / \mathrm{g}]$ & $\mathrm{Cu}^{2+}[\mathrm{mmol} / \mathrm{g}]$ \\
\hline TBZ-Mt-Cu & 25 & $24,5 \pm 0,5$ & $0,121 \pm 0,002$ & $0,28 \pm 0,04$ \\
IMZ-Mt-Cu & 150 & $148 \pm 2$ & $0,498 \pm 0,007$ & $0,27 \pm 0,03$ \\
\hline
\end{tabular}

\subsection{Resultados y discusión}

\subsubsection{Estudios de adsorción}

\subsubsection{Cinéticas de adsorción de IMZ y TBZ en Mt-Cu}

La identificación de las leyes de velocidad que rigen la adsorción de los fungicidas individuales en $\mathrm{Mt}-\mathrm{Cu}$, se realizó cuantificando su concentración en el sobrenadante a diferentes intervalos de tiempo de contacto. Cuando las condiciones experimentales fueron las correspondientes a TBZ-1 e IMZ-1 (Tabla 5.1), se obtuvieron las curvas que se 
muestran en la Figura 5.1. Los valores observados corresponden a la media de tres replicas. En ambos casos, a los primeros 10 min de contacto, más del $98 \%$ de fungicida presente inicialmente en la solución fue removido. Como consecuencia, las concentraciones finales de los fungicidas en el sobrenadante en todo el intervalo de tiempo estuvieron por debajo de 0,2 y 0,4 mg/L para TBZ e IMZ, respectivamente. Teniendo en cuenta que el límite de detección es $0,0045 \mathrm{mg} / \mathrm{L}$ para el primero y $0,117 \mathrm{mg} / \mathrm{L}$ para el segundo, y que los errores asociados a las mediciones superaron esos valores, se consideró que el error introducido en estas condiciones, no permitirían hacer un análisis matemático confiable de los datos, por lo que se procedió a evaluar otras condiciones experimentales.
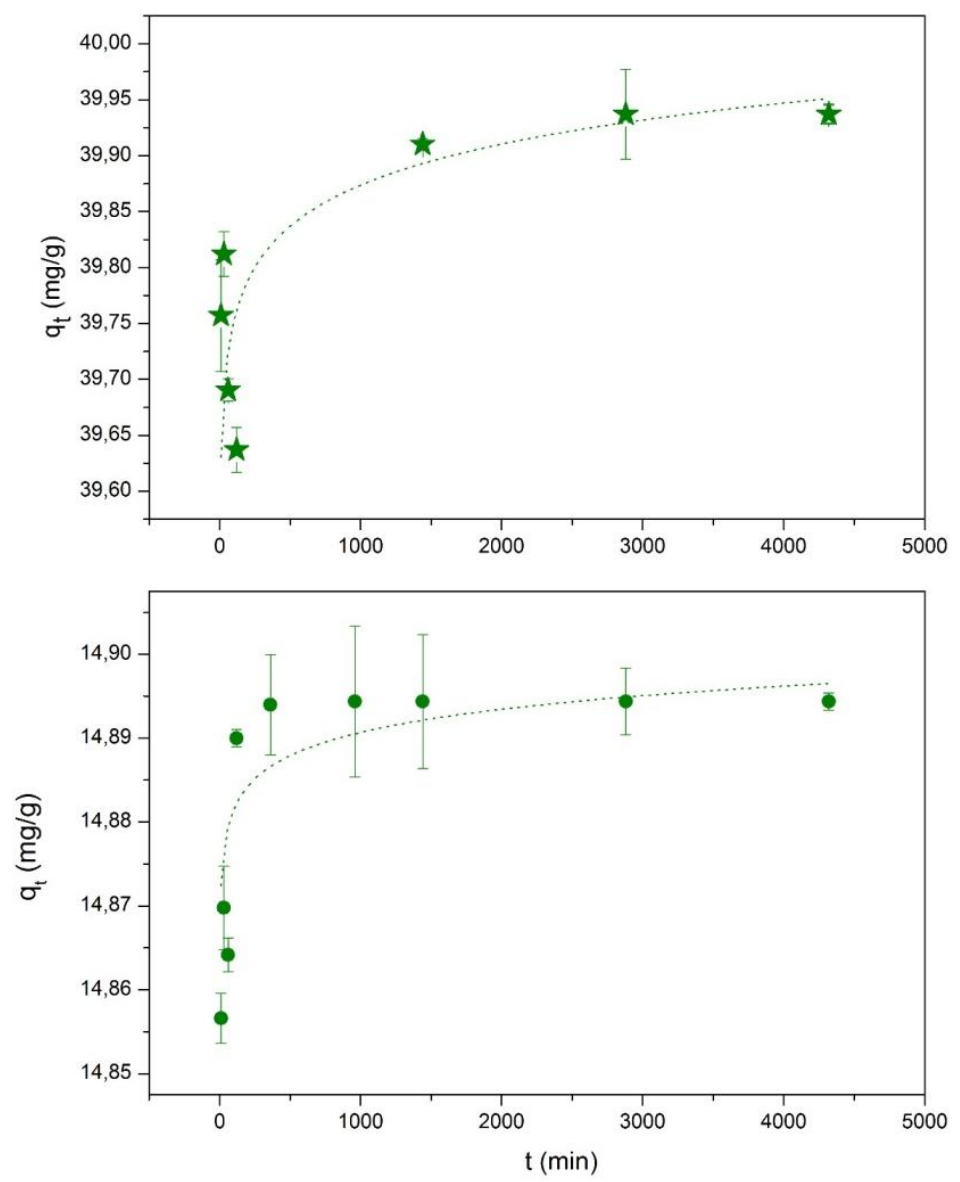

Figura 5.1 Cinéticas de adsorción de $(\star)$ IMZ y de $\bullet$ TBZ en Mt-Cu; relación sólido/solución = $1 \mathrm{~g} / \mathrm{L}$. Las líneas punteadas son un ajuste arbitrario.

Al disminuir la relación sólido/solución a $0,1 \mathrm{~g} / \mathrm{L}$ e incrementar la concentración inicial de los fungicidas (cinéticas TBZ-0,1 e IMZ-0,1; Tabla 5.1) se obtuvieron las gráficas de la Figura 5.2. En ella se observa que las curvas siguen una tendencia exponencial. Entre $t=0$ y $t=100$ min, la velocidad con la que transcurren los procesos (derivada de la curva $q_{t}$ vs. $t$ ) es muy elevada y disminuye posteriormente en forma paulatina hasta alcanzar el equilibrio 
$\left(\mathrm{d} q_{t} / \mathrm{d} t=0\right)$. El proceso de adsorción se puede dar por finalizado luego de 1200 min de contacto para ambos fungicidas, tiempo a partir del cual la concentración final de éstos en el sobrenadante, prácticamente permanece constante (Figura 5.2).
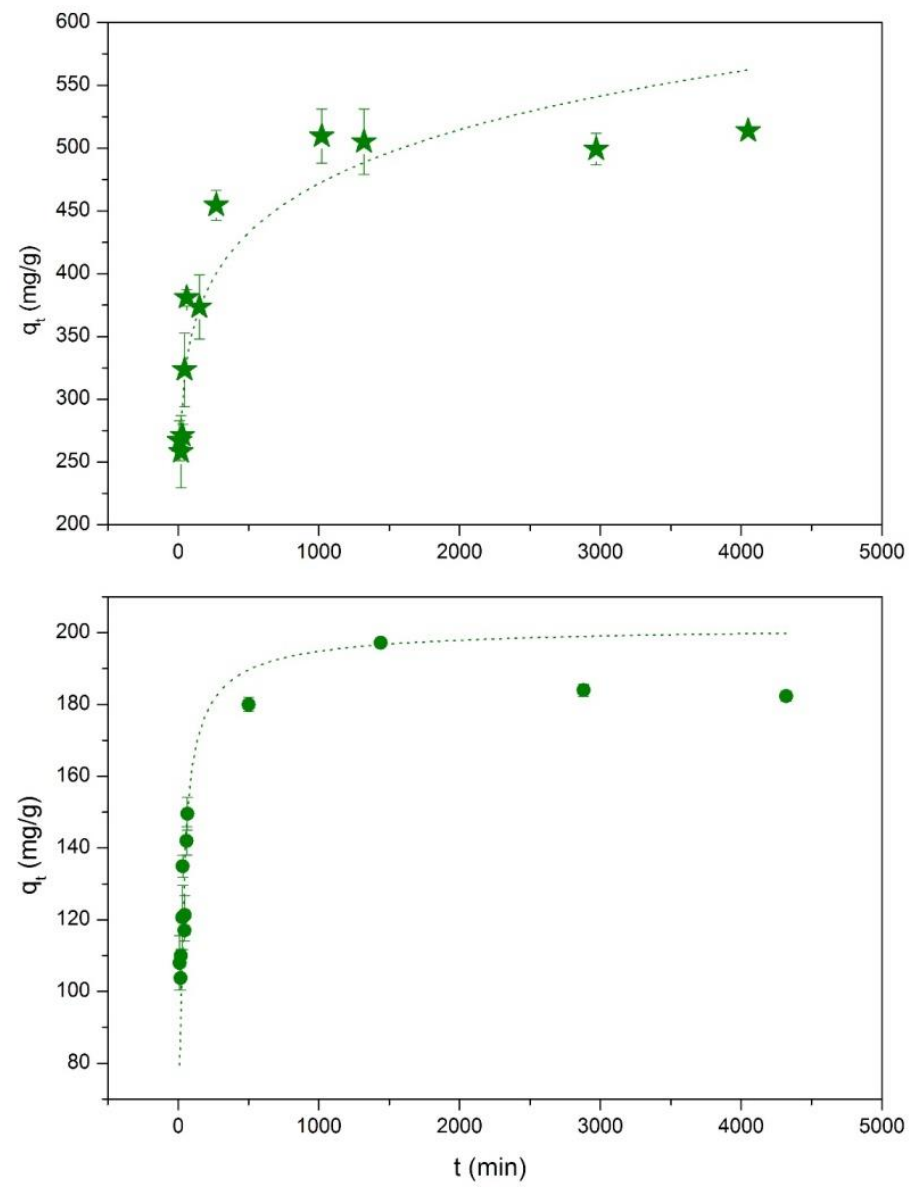

Figura 5.2 Cinéticas de adsorción de ( $\star$ IMZ y de $(\bullet)$ TBZ en Mt-Cu, relación sólido/solución = 0,1 g/L. Las líneas punteadas son un ajuste arbitrario

La Tabla 5.4 muestra los parámetros y el coeficiente de correlación $\mathrm{R}^{2}$ obtenidos tras el ajuste de los diferentes modelos cinéticos a los datos experimentales. Las capacidades de adsorción de los fungicidas individuales en el equilibrio, $q_{e}$, fueron determinadas experimentalmente mediante isotermas de adsorción (Mt-Cu-IMZ-0,1 y Mt-Cu-TBZ-0,1; Tabla 5.2) .

En la Tabla 5.4, puede observarse que el modelo P1O predijo capacidades de adsorción $\left(q_{e, c a l}\right)$ más bajas que las determinadas experimentalmente. Además, este modelo matemático sólo ajustó con los primeros puntos $(0<t<270$ min para IMZ y $0<t<60$ min para

${ }^{8}$ El tiempo que se eligió para llevar a cabo dichas isotermas fue 24 h (1440 min) para garantizar que los sistemas alcancen el equilibrio. 
TBZ). Estos resultados, sumados a los bajos coeficientes de correlación y elevados valores de desviación estándar porcentual obtenidos, indicaron que éste no es modelo adecuado para describir la cinética de adsorción de los fungicidas en Mt-Cu.

Tabla 5.4 Parámetros obtenidos mediante el ajuste de los modelos cinéticos indicados a los datos experimentales.

\begin{tabular}{|c|c|c|c|}
\hline \multirow[t]{2}{*}{ Modelo } & Parámetro & TBZ & IMZ \\
\hline & $q_{e}[\mathrm{mg} / \mathrm{g}]$ & $213 \pm 7$ & $536 \pm 5$ \\
\hline \multirow[t]{4}{*}{$\mathrm{P} 1 \mathrm{O}$} & $q_{e, c a l}[\mathrm{mg} / \mathrm{g}]$ & $119 \pm 3$ & $272 \pm 2$ \\
\hline & $k_{1}[1 / \mathrm{min}]$ & $(8,1 \pm 0,2) \cdot 10^{-3}$ & $(4,4 \pm 0,6) \cdot 10^{-3}$ \\
\hline & $\mathrm{R}^{2}$ & 0,781 & 0,910 \\
\hline & $\Delta q[\%]$ & 23,10 & 11,52 \\
\hline \multirow[t]{5}{*}{$\mathrm{P} 2 \mathrm{O}$} & $q_{e, c a l}[\mathrm{mg} / \mathrm{g}]$ & $184 \pm 6$ & $510 \pm 8$ \\
\hline & $k_{2}[\mathrm{~g} /(\mathrm{mg} \cdot \mathrm{min})]$ & $(1,5 \pm 0,2) 10^{-3}$ & $(6,9 \pm 0,1) \cdot 10^{-5}$ \\
\hline & $b[\mathrm{mg} /(\mathrm{g} \cdot \mathrm{min})]$ & $51 \pm 6$ & $18 \pm 2$ \\
\hline & $\mathrm{R}^{2}$ & 0,999 & 0,999 \\
\hline & $\Delta q[\%]$ & 30,36 & 19,11 \\
\hline \multirow[t]{4}{*}{ Elovich } & $\alpha[\mathrm{mg} /(\mathrm{g} \cdot \mathrm{min})]$ & $1730 \pm 8$ & $997 \pm 6$ \\
\hline & $\beta[\mathrm{g} / \mathrm{mg})]$ & $0,07 \pm 0,04$ & $0,030 \pm 0,002$ \\
\hline & $\mathrm{R}^{2}$ & 0,935 & 0,940 \\
\hline & $\Delta q[\%]$ & 8,46 & 10,34 \\
\hline \multirow[t]{3}{*}{ DIP } & $k^{\prime}[1 / \min ]$ & $(8,1 \pm 0,2) \cdot 10^{-3}$ & $(4,4 \pm 0,6) \cdot 10^{-3}$ \\
\hline & $\mathrm{R}^{2}$ & 0,781 & 0,910 \\
\hline & $\Delta q[\%]$ & 21,06 & 11,52 \\
\hline
\end{tabular}

Por otro lado, el modelo P2O ajustó bien con los datos experimentales en base al valor del coeficiente de correlación $\left(\mathrm{R}^{2}>0,999\right)$ y $q_{e, c a l}\left(\operatorname{cercanos}\right.$ a $q_{\boldsymbol{t}}$, Tabla 5.4). Este modelo fue utilizado satisfactoriamente en el estudio cinético de diversos sistemas sólido/líquido [Gök et al., 2008; Koswojo et al., 2010; Tong et al., 2010; Park et al., 2011; Fatimah y Huda, 2013] y supone que el paso determinante de la velocidad en el proceso es la formación de enlace entre el adsorbato y el adsorbente [Fatimah y Huda, 2013; Jing et al., 2013; Park et 
al., 2013; Wang et al., 2016]. También, el modelo P2O es interpretado por algunos autores como la cinética del modelo de Langmuir [Gupta y Bhattacharyya, 2006] asumiendo, entre otras cosas que el adsorbente presenta sitios de adsorción energéticamente homogéneos (Sección 3.2.3). Es llamativo, sin embargo, que en ambos sistemas dicho modelo presentó los mayores valores de desviación estándar porcentual $(\Delta q)$.

La ecuación de Elovich también ajustó bien a los datos experimentales, teniendo en cuenta el coeficiente de correlación $\mathrm{R}^{2}$ y, más aún, el hecho de que condujo a la desviación estándar porcentual más pequeña $(\Delta q)$. Este modelo, también presupone que el paso determinante es la quimisorción del adsorbato en el adsorbente. A diferencia del modelo $\mathrm{P} 2 \mathrm{O}$, contempla una superficie heterogénea del adsorbente y, por lo tanto, sitios de adsorción con energías de activación diferentes.

Por último, la ecuación de DIP no generó buenos ajustes con los datos experimentales teniendo en cuenta el $\mathrm{R}^{2}$ y debido a que sólo se pudo ajustar en un intervalo de tiempo acotado $(0<t<270$ min para IMZ y $0<t<60$ min para TBZ).

El hecho de que los modelos Elovich y $\mathrm{P} 2 \mathrm{O}$ hayan sido los que mejor se ajustaron con los datos experimentales, indica que los procesos de adsorción de TBZ e IMZ en Mt-Cu están controlados por procesos de quimisorción, es decir, sugiere que los adsorbatos se unen al sólido a través de la formación de enlace y no de procesos físicos. Esta reacción podría ser, basándonos en la hipótesis inicial de este capítulo, la transferencia de electrones desde los átomos de nitrógeno del anillo bencimidazol y tiazol en TBZ e imidazol en IMZ, hacia los orbitales desocupados "d" del cobre, en la formación de un complejo de coordinación.

Tanto en base al modelo de Elovich, como al modelo de P2O, se puede observar que la velocidad del proceso de adsorción de TBZ supera al de adsorción de IMZ, tanto a tiempos cortos (parámetros $h$ en $\mathrm{P} 2 \mathrm{O}$ y $\alpha$ en Elovich) como a tiempos largos (parámetros $k_{2}$ en $\left.\mathrm{P} 2 \mathrm{O}\right)$.

De los dos modelos cinéticos, el de Elovich fue el que condujo a la menor desviación estándar porcentual. Se debe tener en cuenta que, en caso de que la hipótesis fuera correcta y el paso determinante de la velocidad fuera la quelación del $\mathrm{Cu}^{2+}$ por parte de TBZ e IMZ, los resultados son consistentes con el modelo de Elovich que asume la presencia de sitios de adsorción energéticamente diferentes, ya que el metal puede estar unido tanto en los 
sitios de carga permanente, como en los sitios de superficie externa y en menor medida en los sitios de borde?

\subsubsection{Isotermas de adsorción de los sistemas individuales}

Las isotermas de adsorción de los fungicidas individuales en $\mathrm{Mt}$ y $\mathrm{Mt}-\mathrm{Cu}$ en las diferentes condiciones se muestran en las Figuras 5.3 y 5.4. El coeficiente de correlación $\mathrm{R}^{2}$, junto a los parámetros obtenidos mediante el ajuste de los modelos de Freundlich, Langmuir y LF a los datos experimentales, se resumen en las Tablas 5.5 y 5.6.
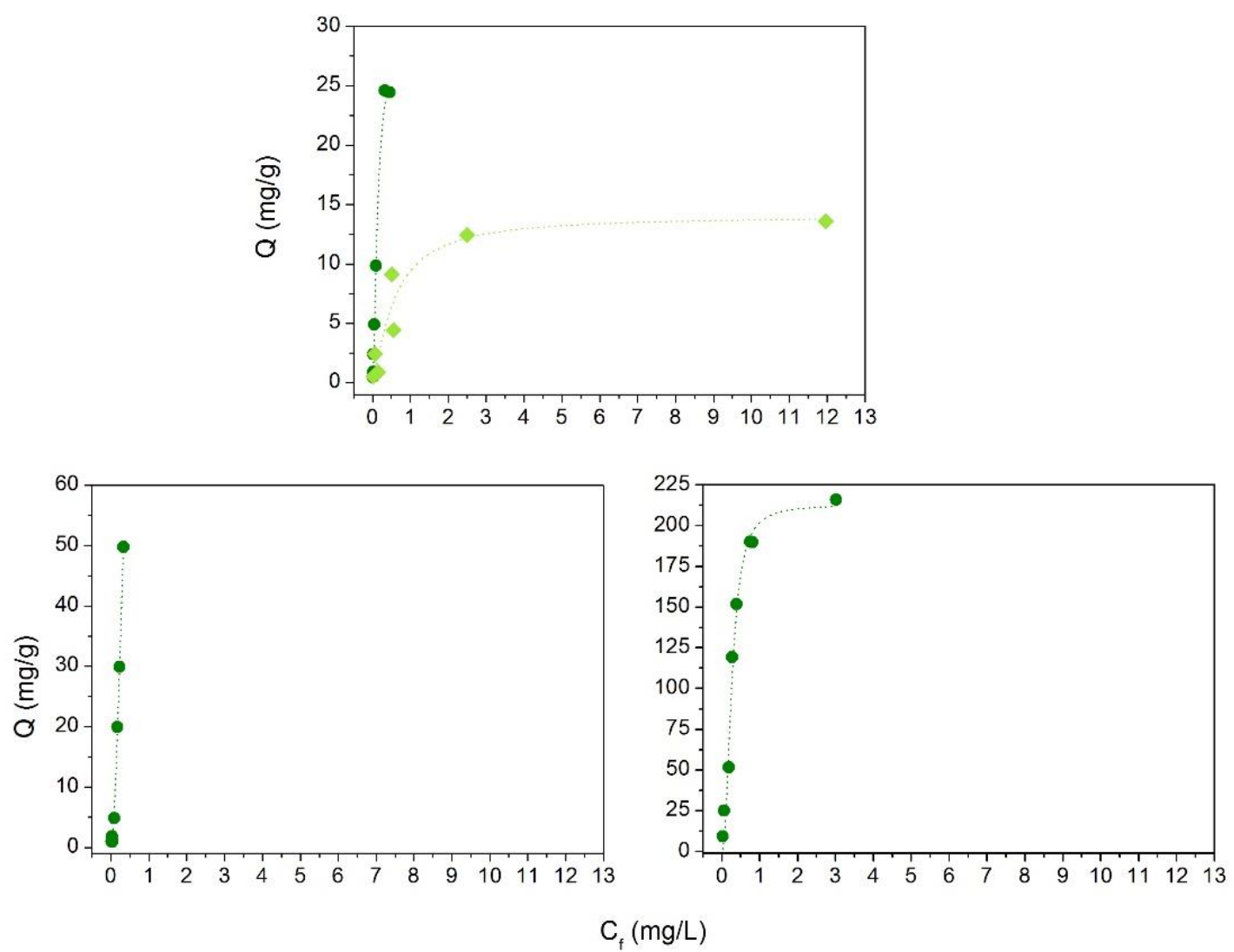

Figura 5.3 Isotermas de adsorción de TBZ en $(\diamond)$ Mt y $(\bullet)$ Mt-Cu. Relación sólido/solución = $1 \mathrm{~g} / \mathrm{L}$ (arriba); $0,5 \mathrm{~g} / \mathrm{L}$ (abajo, izquierda) y 0,1 g/L (abajo, derecha). Las líneas sólidas corresponden al ajuste de LangmuirFreundlich.

El valor de $\mathrm{R}^{2}$ indicó que ni Freundlich ni Langmuir describieron adecuadamente a todos los sistemas. Por un lado, las isotermas Mt-TBZ-1, Mt-Cu-TBZ-0,1 y Mt-Cu-IMZ0,5 presentaron $\mathrm{R}^{2}<0,8$ cuando fueron ajustadas al modelo de Freundlich (Tablas 5.5 y 5.6). Por otro lado, las isotermas Mt-TBZ-1 y Mt-Cu-IMZ-0,5 presentaron $\mathrm{R}^{2}<0,9$ cuando se ajustaron a Langmuir. Además, las isotermas Mt-Cu-TBZ-1 y Mt-Cu-TBZ-0,5

${ }^{9} \mathrm{La}$ síntesis de $\mathrm{Mt}-\mathrm{Cu}$ se llevó a cabo a $\mathrm{pH}=4,8$. Teniendo en cuenta que el $\mathrm{pH}_{\text {PZC,borde }}$ está entre 4,2 y 5,3 es probable que los bordes se encuentren parcialmente con carga positiva al $\mathrm{pH}$ de la síntesis. 
presentaron parámetros de Langmuir $\left(Q_{\max } \mathrm{y} k\right)$ con errores asociados del mismo orden de magnitud o superior cuando fueron ajustadas a este modelo (Tablas 5.5 y 5.6), lo cual también evidenció que estos sistemas no cumplen con las suposiciones del modelo monocapa y de sitios de adsorción homogéneos que asume esa ecuación.
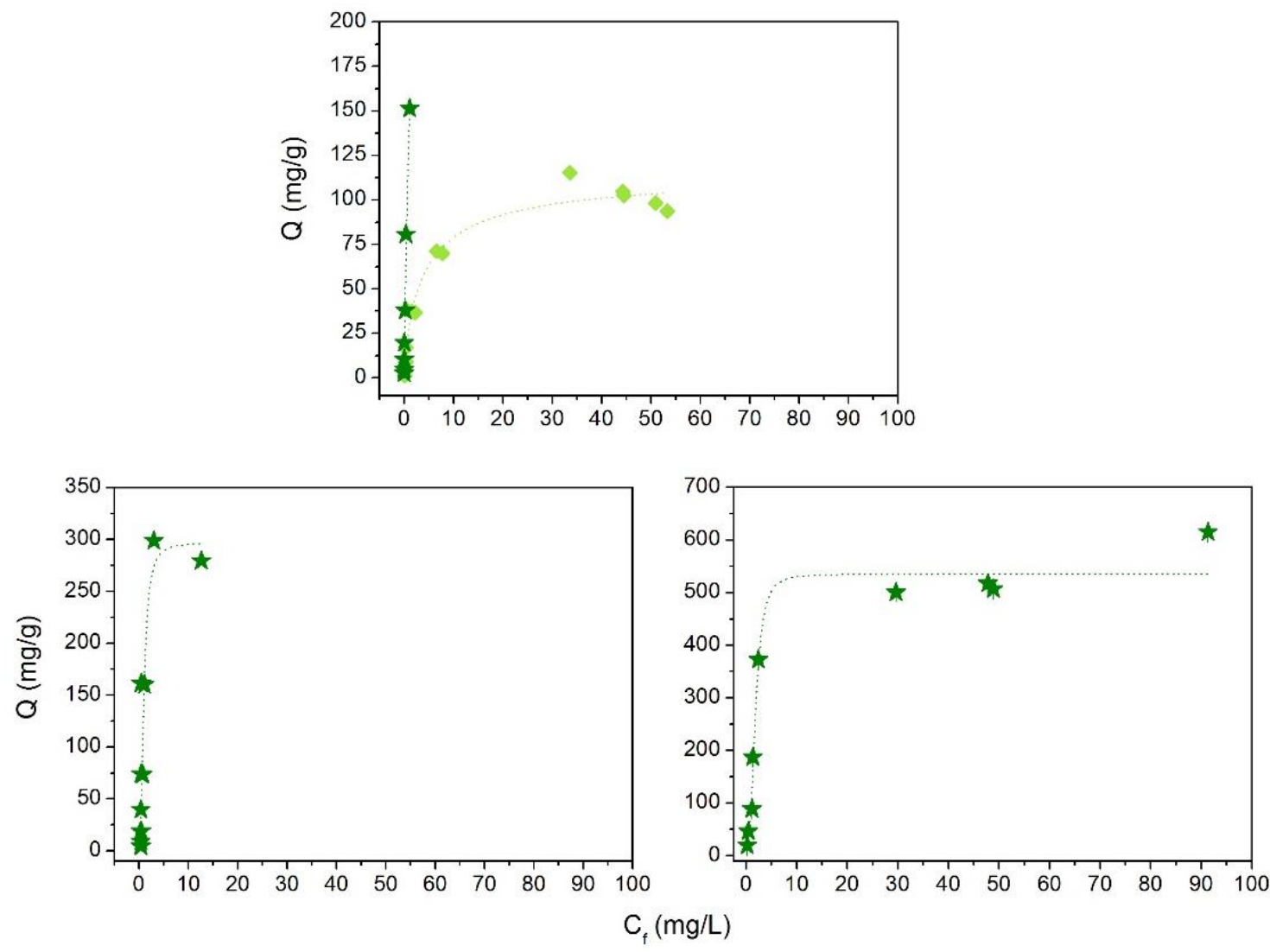

Figura 5.4 Isotermas de adsorción de IMZ en $(\star)$ Mt y ( $\star$ ) Mt-Cu. Relación sólido/solución = $1 \mathrm{~g} / \mathrm{L}$ (arriba); $0,5 \mathrm{~g} / \mathrm{L}$ (abajo, izquierda) y 0,1 g/L (abajo, derecha). Las líneas sólidas corresponden al ajuste de LangmuirFreundlich.

Estos resultados están en concordancia con los resultados de los estudios cinéticos, donde el modelo cinético de Langmuir -P2O- fue descartado por conducir a una desviación estándar porcentual muy elevada. Como se mencionó entonces, por las características del adsorbente $y$, en particular, la multiplicidad de sitios que presenta, la heterogeneidad de la superficie debe ser tenida en cuenta en los modelos matemáticos. En ese sentido la ecuación LF contiene un parámetro que da cuenta de ello $(1 / n)$. Este fue el único modelo que presentó $\mathrm{R}^{2}>0,9$ para todos los sistemas $\mathrm{y}$, por lo tanto, fue el elegido para comparar el desempeño de los adsorbentes con los fungicidas y/o sus mezclas.

La capacidad de adsorción en el equilibrio por unidad de masa $\left(Q_{\max , S}\right)$ de todas las muestras disminuyó considerablemente al aumentar la relación sólido/solución de acuerdo 
Tabla 5.5 Parámetros obtenidos mediante el ajuste de los modelos de Freundlich, Langmuir y LangmuirFreundlich a las isotermas de adsorción de TBZ en Mt-Cu.

\begin{tabular}{|c|c|c|c|c|c|}
\hline \multirow{2}{*}{\multicolumn{2}{|c|}{$\begin{array}{l}\text { Adsorbato } \\
\text { Isoterma }\end{array}$}} & \multicolumn{4}{|c|}{ TBZ } \\
\hline & & \multirow{2}{*}{$\frac{\text { Mt-TBZ-1 }}{7 \pm 1}$} & \multirow{2}{*}{$\frac{\text { Mt-Cu-TBZ-1 }}{47 \pm 7}$} & \multirow{2}{*}{$\frac{\text { Mt-Cu-TBZ-0,5 }}{(21 \pm 5) \cdot 10^{1}}$} & \multirow{2}{*}{$\frac{\text { Mt-Cu-TBZ-0,1 }}{(17 \pm 2) \cdot 10^{1}}$} \\
\hline \multirow{3}{*}{ Freundlich } & $K_{f}[\mathrm{~L} / \mathrm{mg}]$ & & & & \\
\hline & $1 / n$ & $0,31 \pm 0,09$ & $0,9 \pm 0,1$ & $1,3 \pm 0,2$ & $0,36 \pm 0,09$ \\
\hline & $\mathrm{R}^{2}$ & 0,789 & 0,965 & 0,974 & 0,757 \\
\hline \multirow{3}{*}{ Langmuir } & $Q_{\max }[\mathrm{mg} / \mathrm{g}]$ & $15 \pm 2$ & $(2 \pm 6) \cdot 10^{2}$ & $(1 \pm 2) \cdot 10^{6}$ & $(26 \pm 3) \cdot 10^{1}$ \\
\hline & $k[\mathrm{~L} / \mathrm{g}]$ & $1,5 \pm 0,7$ & $0,3 \pm 0,8$ & $(1 \pm 3) \cdot 10^{-3}$ & $2,7 \pm 0,8$ \\
\hline & $\mathrm{R}^{2}$ & 0,879 & 0,953 & 0,941 & 0,931 \\
\hline \multirow{6}{*}{ LF } & $Q_{\max , S}[\mathrm{mg} / \mathrm{g}]$ & $14 \pm 2$ & $28 \pm 2$ & $(15 \pm 8) \cdot 10^{1}$ & $(21 \pm 1) \cdot 10^{1}$ \\
\hline & $Q_{\max , S}[\mathrm{mmol} / \mathrm{g}]$ & $0,065 \pm 0,009$ & $0,099 \pm 0,007$ & $0,7 \pm 0,4$ & $0,104 \pm 0,005$ \\
\hline & $K_{s}[\mathrm{~L} / \mathrm{mg}]$ & $1,7 \pm 0,8$ & $8 \pm 1$ & $2,2 \pm 0,1$ & $3,7 \pm 0,3$ \\
\hline & $K_{s}[\mathrm{~L} / \mathrm{mmol}]$ & $(3 \pm 1) \cdot 10^{2}$ & $(14 \pm 2) \cdot 10^{2}$ & $(44 \pm 2) \cdot 10^{1}$ & $(74 \pm 6) \cdot 10^{1}$ \\
\hline & $1 / n$ & $1,2 \pm 0,7$ & $1,7 \pm 0,3$ & $1,8 \pm 0,3$ & $2,2 \pm 0,5$ \\
\hline & $\mathrm{R}^{2}$ & 0,920 & 0,993 & 0,997 & 0,982 \\
\hline
\end{tabular}

Tabla 5.6 Parámetros obtenidos mediante el ajuste de los modelos de Freundlich, Langmuir y LangmuirFreundlich a las isotermas de adsorción de IMZ en Mt-Cu.

\begin{tabular}{|c|c|c|c|c|c|}
\hline \multirow{2}{*}{\multicolumn{2}{|c|}{$\frac{\text { Adsorbato }}{\text { Isoterma }}$}} & \multicolumn{4}{|c|}{ IMZ } \\
\hline & & Mt-IMZ-1 & Mt-Cu-IMZ-1 & Mt-Cu-IMZ-0,5 & Mt-Cu-IMZ-0,1 \\
\hline \multirow{3}{*}{ Freundlich } & $K_{f}[\mathrm{~L} / \mathrm{mg}]$ & $28 \pm 4$ & $139 \pm 9$ & $87 \pm 24$ & $(54 \pm 2) \cdot 10^{1}$ \\
\hline & $1 / n$ & $0,3 \pm 0,3$ & $0,7 \pm 0,1$ & $0,6 \pm 0,1$ & $0,31 \pm 0,06$ \\
\hline & $\mathrm{R}^{2}$ & 0,922 & 0,962 & 0,782 & 0,874 \\
\hline \multirow{3}{*}{ Langmuir } & $Q_{\max }[\mathrm{mg} / \mathrm{g}]$ & $108 \pm 4$ & $(33 \pm 9) \cdot 10^{1}$ & $(38 \pm 9) \cdot 10^{1}$ & $(54 \pm 2) \cdot 10^{1}$ \\
\hline & $k[\mathrm{~L} / \mathrm{g}]$ & $0,33 \pm 0,06$ & $0,7 \pm 0,3$ & $0,4 \pm 0,2$ & $0,3 \pm 0,1$ \\
\hline & $\mathrm{R}^{2}$ & 0,961 & 0,974 & 0,826 & 0,936 \\
\hline \multirow{6}{*}{$\mathrm{LF}$} & $Q_{\max , S}[\mathrm{mg} / \mathrm{g}]$ & $(12 \pm 1) \cdot 10^{1}$ & $(29 \pm 1) \cdot 10^{1}$ & $(40 \pm 7) \cdot 10^{1}$ & $(54 \pm 2) \cdot 10^{1}$ \\
\hline & $Q_{\max , S}[\mathrm{mmol} / \mathrm{g}]$ & $0,40 \pm 0,03$ & $0,98 \pm 0,03$ & $1,3 \pm 0,2$ & $1,8 \pm 0,07$ \\
\hline & $K_{s}[\mathrm{~L} / \mathrm{mg}]$ & $0,25 \pm 0,09$ & $1,42 \pm 0,06$ & $0,52 \pm 0,07$ & $0,56 \pm 0,06$ \\
\hline & $K_{s}[\mathrm{~L} / \mathrm{mmol}]$ & $(7 \pm 3) \cdot 10^{1}$ & $(42 \pm 2) \cdot 10^{1}$ & $(15 \pm 2) \cdot 10^{1}$ & $(17 \pm 2) \cdot 10^{1}$ \\
\hline & $1 / n$ & $0,8 \pm 0,1$ & $4,0 \pm 0,5$ & $0,94 \pm 0,03$ & $2,7 \pm 0,7$ \\
\hline & $\mathrm{R}^{2}$ & 0,964 & 0,986 & 0,968 & 0,962 \\
\hline
\end{tabular}

con lo observado para otros sistemas [Fruhstorfer et al., 1993; Lombardi et al, 2003; Kaya y Oren, 2005] y este comportamiento se asignó a un incremento de la cantidad de sitios activos que permanecen insaturados durante la adsorción a cantidades altas de adsorbente en la suspensión [Akpomie y Dawodu, 2014] (una discusión más profunda al respecto se presentará en el Capítulo 6). A pesar de que este parámetro es una extrapolación, porque 
no siempre es posible alcanzar un plateau, puede ser utilizado con fines comparativos. Tanto para TBZ como para IMZ, la presencia de $\mathrm{Cu}^{2+}$ en la estructura llevó a un incremento de $Q_{\max , S}$ respecto a la adsorción en Mt (Tablas 5.5 y 5.6). Más aún, la constante de afinidad $K_{s}$ -una medida del nivel de interacciones entre el adsorbato y la superficie sólida- también fue mayor para las muestras con $\mathrm{Cu}^{2+}$ (Tablas 5.5 y 5.6). Este resultado indica que el metal está involucrado en la interacción con los fungicidas.

El intercambio catiónico que se propuso como mecanismos de adsorción de IMZ y TBZ en Mt (Secciones 3.3.2 y 4.3.2) se descarta por dos motivos. Por un lado, por la constancia de $\mathrm{pH}$ durante la adsorción: es necesario recordar que durante la adsorción de IMZ o TBZ en Mt, la protonación del fungicida en la superficie del adsorbente o en la solución, iba acompañada de la liberación de $\mathrm{OH}^{-}$(Figura 3.10, Ecuación 4.1) que se traducía en un incremento de $\mathrm{pH}$ en los experimentos de adsorción. El hecho de que el pH no cambió en el tiempo cuando se utilizó $\mathrm{Mt}-\mathrm{Cu}$ como adsorbente, indica que no se desarrollaron procesos ácidos-base durante las reacciones de adsorción. Por otro lado, los datos de absorción atómica: el contenido de $\mathrm{Cu}^{2+}$ que se liberó durante la adsorción en $\mathrm{meq} / \mathrm{g}$ es un orden de magnitud menor que la cantidad de fungicida adsorbida en $\mathrm{mmol} / \mathrm{g}$ (Tabla 5.3).

Para comparar el desempeño del adsorbente entre los fungicidas, es necesario expresar los parámetros $Q_{\max , S}$ y $K_{s}$ en $[\mathrm{mmol} / \mathrm{g}]$ y $[\mathrm{L} / \mathrm{mmol}]$. Al comparar estos valores (Tablas $5.5 \mathrm{y}$ 5.6), se observa que $Q_{\max , S}$ fue mayor para IMZ que para TBZ; mientras que $K_{s}$ fue superior en TBZ que en IMZ. Esto indica que la cantidad de sitios activos en Mt-Cu para la adsorción de IMZ es mayor que para la adsorción de TBZ. Al mismo tiempo, el adsorbente presenta mayor afinidad por el último que por el primero.

\subsubsection{Isotermas de adsorción múltiples}

En la Figura 5.5 se grafican las isotermas de adsorción de los fungicidas individuales (Mt-Cu-TBZ-0,1 y Mt-Cu-IMZ-0,1) y de los fungicidas combinados en forma simultánea (Mt-Cu-TBZ(Sim) y Mt-Cu-IMZ(Sim)) y consecutiva (Mt-Cu-TBZ(Con) y Mt-CuIMZ(Con)) con sus respectivos ajustes LF, con el objetivo de comparar el efecto de la presencia de un segundo compuesto en la adsorción. Luego, en la Tabla 5.7 se muestran los parámetros obtenidos mediante el ajuste matemático de LF a los datos experimentales.

La presencia de un segundo compuesto, ya sea previamente adsorbido o en la misma suspensión, generó una disminución de $Q$ para todo el intervalo de $C_{f}$, tanto en la adsorción de TBZ como de IMZ (Figuras 5.3, 5.4 y 5.5). Esto, a priori, permitiría descartar efectos 
sinérgicos, y por lo contrario plantearía la existencia de sitios de adsorción comunes para ambos fungicidas [Fan et al., 2014].

Una diferencia encontrada, es que en las isotermas de adsorción de IMZ, $Q$ y $Q_{\max , S}$ siguieron la tendencia: Mt-Cu-IMZ-0,1 > Mt-Cu-IMZ(Con) > Mt-Cu-IMZ(Sim) (es decir, la cantidad adsorbida fue mayor en la isoterma individual, luego en la combinada consecutivamente y luego en la combinada simultáneamente); mientras que en las isotermas
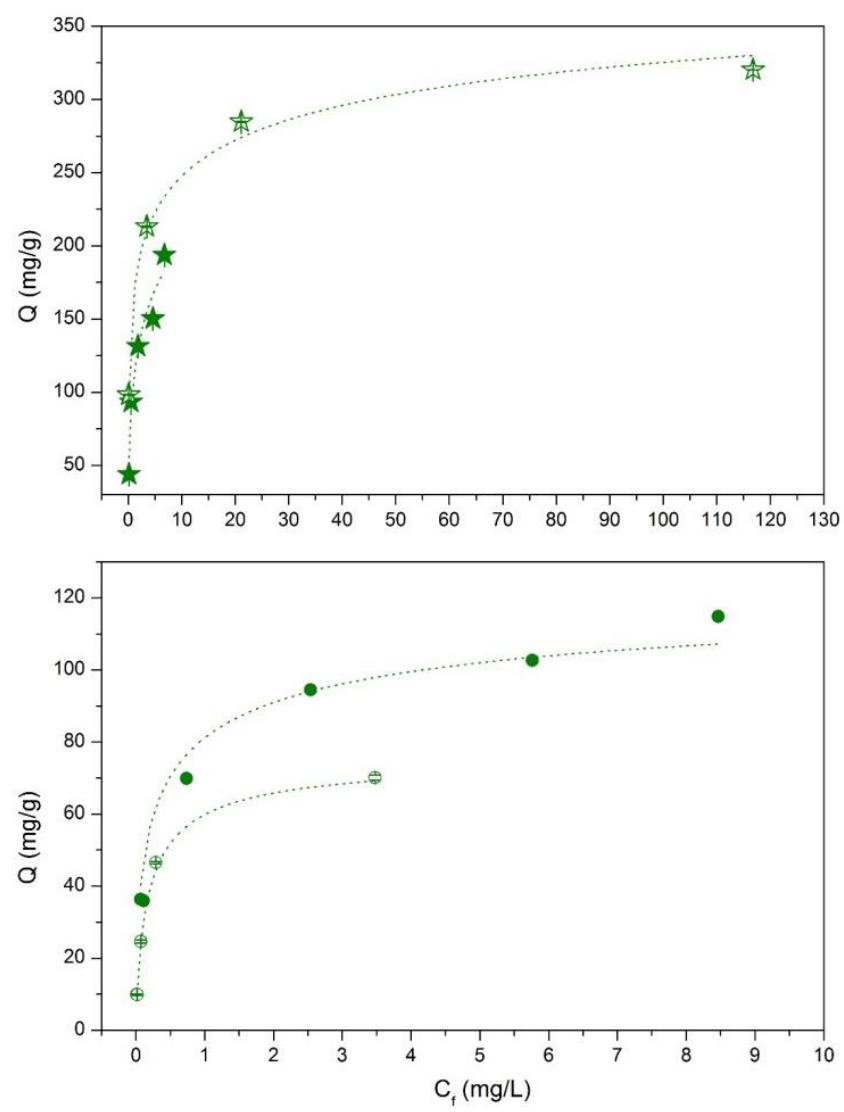

Figura 5.5 Isotermas de adsorción múltiples. Los símbolos indican: (放) Mt-Cu-IMZ(Con), ( $\star$ ) Mt-CuIMZ(Sim), (○) Mt-Cu-TBZ(Con) y (•) Mt-Cu-TBZ(Sim). Las líneas corresponden al ajuste de LF.

Tabla 5.7 Parámetros obtenidos mediante el ajuste de Langmuir-Freundlich a las isotermas de adsorción múltiples de TBZ e IMZ en Mt-Cu.

\begin{tabular}{|c|c|c|c|c|}
\hline Adsorbato & \multicolumn{2}{|c|}{ TBZ } & \multicolumn{2}{c|}{ IMZ } \\
\hline Isoterma & Mt-Cu-TBZ(Sim) & Mt-Cu-TBZ(Con) & Mt-Cu-IMZ(Sim) & Mt-Cu-IMZ(Con) \\
\hline$Q_{\text {max }, S}[\mathrm{mg} / \mathrm{g}]$ & $(13 \pm 5) \cdot 10^{1}$ & $77 \pm 3$ & $(35 \pm 5) \cdot 10^{1}$ & $(4,7 \pm 0,5) \cdot 10^{2}$ \\
$Q_{\text {max }, S}[\mathrm{mmol} / \mathrm{g}]$ & $0,06 \pm 0,02$ & $0,4 \pm 0,1$ & $0,12 \pm 0,02$ & $1,6 \pm 0,2$ \\
$K_{s}[\mathrm{~L} / \mathrm{g}]$ & $2,8 \pm 0,6$ & $5,2 \pm 0,8$ & $0,17 \pm 0,09$ & $0,14 \pm 0,03$ \\
$K_{s}[\mathrm{~L} / \mathrm{mmol}]$ & $(6 \pm 1) \cdot 10^{2}$ & $(10 \pm 1) \cdot 10^{2}$ & $(5 \pm 2) \cdot 10^{1}$ & $42 \pm 9$ \\
$1 / n$ & $0,48 \pm 0,03$ & $0,77 \pm 0,06$ & $0,47 \pm 0,03$ & $0,30 \pm 0,09$ \\
$\mathrm{R}^{2}$ & 0,902 & 0,995 & 0,960 & 0,969 \\
\hline
\end{tabular}


de adsorción de TBZ, $Q$ y $Q_{\max , S}$ siguieron el orden Mt-Cu-TBZ-0,1 > Mt-Cu-TBZ(Sim) > Mt-Cu-TBZ(Con) (es decir, la cantidad adsorbida fue mayor en la isoterma individual, luego en combinada simultáneamente y luego en la combinada consecutivamente). Para comprender este fenómeno, es necesario tener en cuenta que la relación fungicida/ $\mathrm{Cu}^{2+}$ (en mmoles) en los productos de adsorción fue 1,84 en Mt-Cu-IMZ y 0,43 en Mt-Cu-TBZ (Tabla 5.3). Es decir, idealmente todos los iones $\mathrm{Cu}^{2+}$ están ocupados en Mt-Cu-IMZ por lo que la adsorción posterior de TBZ está limitada. Por el contrario, sólo un 40\% de los sitios están ocupado en Mt-Cu-TBZ, con lo cual aún quedan disponibles el 60\% de los cationes como sitios activos para unirse al IMZ. La remoción de fungicida pre-adsorbido en cada caso, fue menor a $1,5.10^{-3} \mathrm{mmol} / \mathrm{g}$, postulando la posibilidad de utilizar un mismo adsorbente en el tratamiento consecutivo de efluente con distintos fungicidas.

Al comparar las isotermas obtenidas con los fungicidas combinados en simultáneo (isotermas $\mathrm{Mt}-\mathrm{Cu}-\mathrm{TBZ}(\mathrm{Sim})$ y $\mathrm{Mt}-\mathrm{Cu}-\mathrm{IMZ}(\mathrm{Sim})$ ) se observa que cuando ambos fungicidas están presentes en la solución inicial en relación equimolar, el adsorbente presenta mayor capacidad de adsorción de IMZ que de TBZ, lo cual condujo a mayores valores de $Q_{\max , S}$ (Tabla 5.7). Esto es llamativo ya que la adsorción de TBZ está favorecida respecto a la de IMZ desde el punto de vista cinético (presentó mayores constantes de velocidad, Tabla 5.4). Sin embargo, como se está trabajando en condiciones de equilibrio, poco importa la dependencia de la adsorción con el tiempo. La adsorción de los compuestos en el equilibrio dependen de las características del adsorbente y del adsorbato, de las interacciones adsorbato-adsorbente, solvente-adsorbato y solvente-adsorbente, así como de las condiciones del sistema ( $\mathrm{pH}$, temperatura, etc). La distribución diferencial de las moléculas de soluto o iones entre las fases líquida y sólida resulta de su afinidad relativa para cada fase que a su vez se relaciona con la naturaleza de las interacciones anteriormente mencionadas. La presencia del segundo compuesto en la solución evidentemente afecta esas afinidades relativas lo cual se expresa en el cambio del valor de $K_{s}$. La constante de afinidad en las isotermas combinadas, al igual que en las individuales, es un orden de magnitud mayor para TBZ que para IMZ. La tendencia que sigue $K_{s}$ para las isotermas de adsorción de TBZ es Mt-Cu-TBZ(Con) > Mt-Cu-TBZ-0,1 > Mt-Cu-TBZ(Sim); mientras que para IMZ es MtCu-IMZ-0,1> Mt-Cu-IMZ(Sim) $\approx$ Mt-Cu-IMZ(Con). Es decir, la presencia de TBZ hace disminuir la afinidad del adsorbente por IMZ, mientras que la presencia del IMZ previamente adsorbido en $\mathrm{Mt}-\mathrm{Cu}$ aumenta la afinidad del adsorbente por el TBZ. Esto no se cumple cuando el IMZ se encuentra en la misma suspensión lo que condujo a una disminución de $K_{s}$ para TBZ respecto a su isoterma individual. 
Resumiendo, se podría decir entonces, que el número de sitios disponibles para la adsorción de IMZ en Mt-Cu es mayor que para la adsorción de TBZ. Probablemente el IMZ actúa como ligando monodentado (al igual que su precursor, el imidazol [Holešová et al., 2009]). Si esto fuera así, teóricamente podrían unirse hasta seis moléculas por ion $\mathrm{Cu}^{2+}$. El TBZ, por el contrario es un ligando bidentado [Grevy et al., 2002], con lo cual el $\mathrm{Cu}^{2+}$ podría aceptar sólo tres moléculas. Otra posible explicación podría ser que, además de la coordinación entre el átomo de nitrógeno del imidazol y el metal, se establezcan interacciones hidrofóbicas o $\pi-\pi$ entre los anillos [Lelario et al., 2016].

\subsubsection{Caracterización de los materiales}

La incorporación de $\mathrm{Cu}^{2+}$ en la intercapa de Mt produjo un valor de espaciado basal correspondiente a 1,25 nm que se asignó a la presencia de complejos hexa-acuo $\left[\mathrm{Cu}\left(\mathrm{H}_{2} \mathrm{O}\right)_{6}\right]^{2+}[$ Brtáňová et al., 2014; Joseph-Ezra et al., 2014] (Figura 5.6). La adsorción de TBZ en Mt-Cu movió el pico de reflexión $\mathrm{d}_{001}$ a valores $2 \theta$ más bajos, lo que evidenció su entrada al espacio interlaminar. Como se indicó en el Capítulo 4, la adsorción de TBZ en Mt llevó a un espacio interlaminar de 1,51 nm que correspondió a una orientación paralela del compuesto respecto a las láminas T:O:T [Lombardi et al., 2003; Roca Jalil et al., 2013].

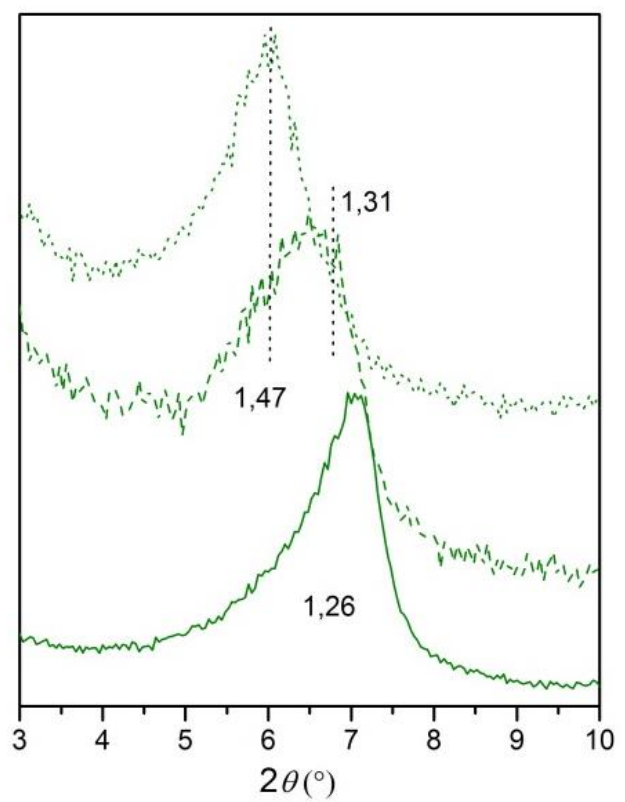

Figura 5.6 Difractogramas con el espaciado basal indicado (en nm) de (-) Mt-Cu, (---) TBZ-Mt-Cu y ( $\cdots$ ) IMZ-Mt-Cu.

Sin embargo, para la muestra $\mathrm{Mt}-\mathrm{Cu}$ se obtuvo un ensanchamiento del pico, cuya deconvolución sugirió la existencia de dos valores $\mathrm{d}_{001}$. El primero, de 1,47 nm, se atribuyó 
al complejo TBZ-Cu ${ }^{2+}$ que se formó con desplazamiento de moléculas de agua de la esfera de hidratación del metal en la intercapa, lo que provocó una contracción de la distancia interlaminar de 0,04 nm en Mt-Cu-TBZ respecto a la muestra Mt-TBZ. El segundo pico, correspondió a 1,31 nm y se asignó $\left[\mathrm{Cu}\left(\mathrm{H}_{2} \mathrm{O}\right)_{6}\right]^{2+}$ que permanecen en los sitios de carga permanente sin interactuar con el TBZ [Brtáňová et al., 2014; Joseph-Ezra et al., 2014]. Es importante notar que, como se mencionó en párrafos más arriba, la relación TBZ/ $\mathrm{Cu}^{2+}$ (en mmoles) en el producto de adsorción fue 0,43 (Tabla 5.3). Este valor indica que, si cada molécula de TBZ se encontrara unida a un catión $\mathrm{Cu}^{2+}$, quedarían aun 0,19 $\mathrm{mmol} / \mathrm{g}$ de $\mathrm{Cu}^{2+}$ en forma de complejos hexa-acuo provocando los dos valores $\mathrm{d}_{001}$ observados.

En el caso del fungicida IMZ, la adsorción en Mt, generó un pico $\mathrm{d}_{001}$ que correspondió a un espaciado basal de 1,82 nm (Sección 3.3.3), mientras que el espaciado que originó al intercalarse en $\mathrm{Mt}-\mathrm{Cu}$ fue de 1,47 $\mathrm{nm}$. Esta diferencia de 0,35 $\mathrm{nm}$ se asignó a la diferencia en cantidad adsorbida en un producto respecto del otro (en el Capítulo 3 se presentaron caracterizaciones de productos de adsorción obtenidos con $C_{i}(\mathrm{IMZ})=600 \mathrm{mg} / \mathrm{L}$, mientras que en el presente capítulo, se trabajó con $\left.C_{i}(\mathrm{IMZ})=150 \mathrm{mg} / \mathrm{L}\right)$. La relación $\mathrm{IMZ} / \mathrm{Cu}^{2+}$ en mmoles fue 1,84; esto significa que si todas las moléculas de IMZ se ubicaran en la intercapa, todos los cationes $\mathrm{Cu}^{2+}$ estarían enlazados al ligando (algunos hasta a dos moléculas IMZ). Así podría explicarse la desaparición del pico correspondiente al complejo hexa-acuo.

Las curvas de potencial zeta vs. $\mathrm{pH}$ del adsorbente $(\mathrm{Mt}-\mathrm{Cu})$ y de los productos de adsorción TBZ-Mt-Cu e IMZ-Mt-Cu, se muestran en la Figura 5.7. El intercambio catiónico con $\mathrm{Cu}^{2+}$, al igual que la adsorción de TBZ e IMZ en Mt (Figura 5.7, Secciones 3.3.2 y 4.3.2) promovieron un aumento de potencial zeta en todo el intervalo de $\mathrm{pH}$ lo que indicó su presencia en los sitios de superficie externa. Esto ya se explicó en secciones anteriores y está directamente relacionado con la unión electrostática de los cationes $\left(\mathrm{Cu}^{2+}\right.$, $\mathrm{IMZH}^{+}$o $\mathrm{TBZH}^{+}$) en la superficie externa de la Mt. Sin embargo, un comportamiento diferente mostraron los productos de adsorción de IMZ y TBZ en $\mathrm{Mt}-\mathrm{Cu}$, donde la curva de potencial zeta vs. pH se corrió hacia valores más negativos. Es probable que esto se deba a la desorción de $\mathrm{Cu}^{2+}$ de los sitios de superficie externa o de borde durante el ensayo de adsorción de los fungicidas, y es coherente con la disminución del contenido de $\mathrm{Cu}^{2+}$ en los productos de adsorción respecto al material de partida $(0,28 \mathrm{mmol} / \mathrm{g}$ en TBZ-Mt-Cu; $0,27 \mathrm{mmol} / \mathrm{g}$ en IMZ-Mt-Cu y 0,309 $\mathrm{mmol} / \mathrm{g}$ en $\mathrm{Mt}-\mathrm{Cu}$ ). Igualmente, la explicación a este fenómeno sigue siendo investigada. 


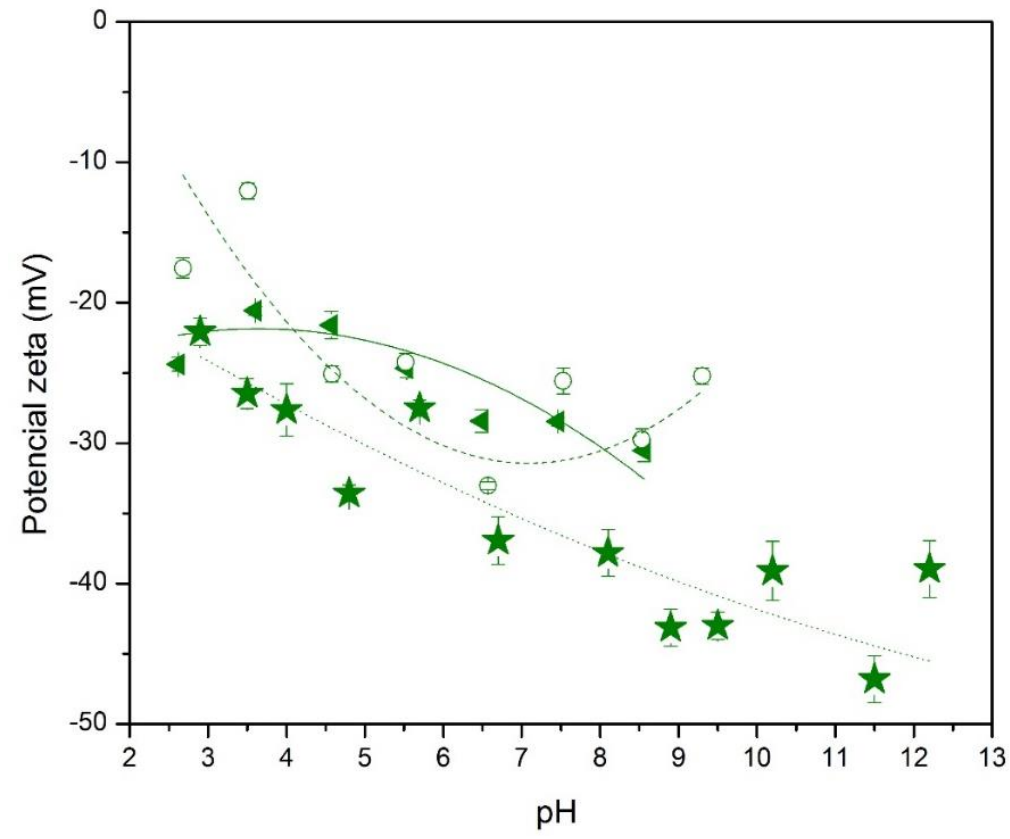

Figura 5.7 Curvas de potencial zeta vs. pH de $(<,-)$ Mt-Cu, $(\star, \cdots)$ IMZ-Mt-Cu y (०, ---) TBZ-Mt-Cu. Las curvas son regresiones lineales utilizando una ecuación polinomial, con el objetivo de facilitar el seguimiento de los puntos correspondientes a cada muestra.

La Figura 5.8 y la Tabla 5.8 presentan los datos de Tg, DTg y ATD del adsorbente y de los productos de adsorción.
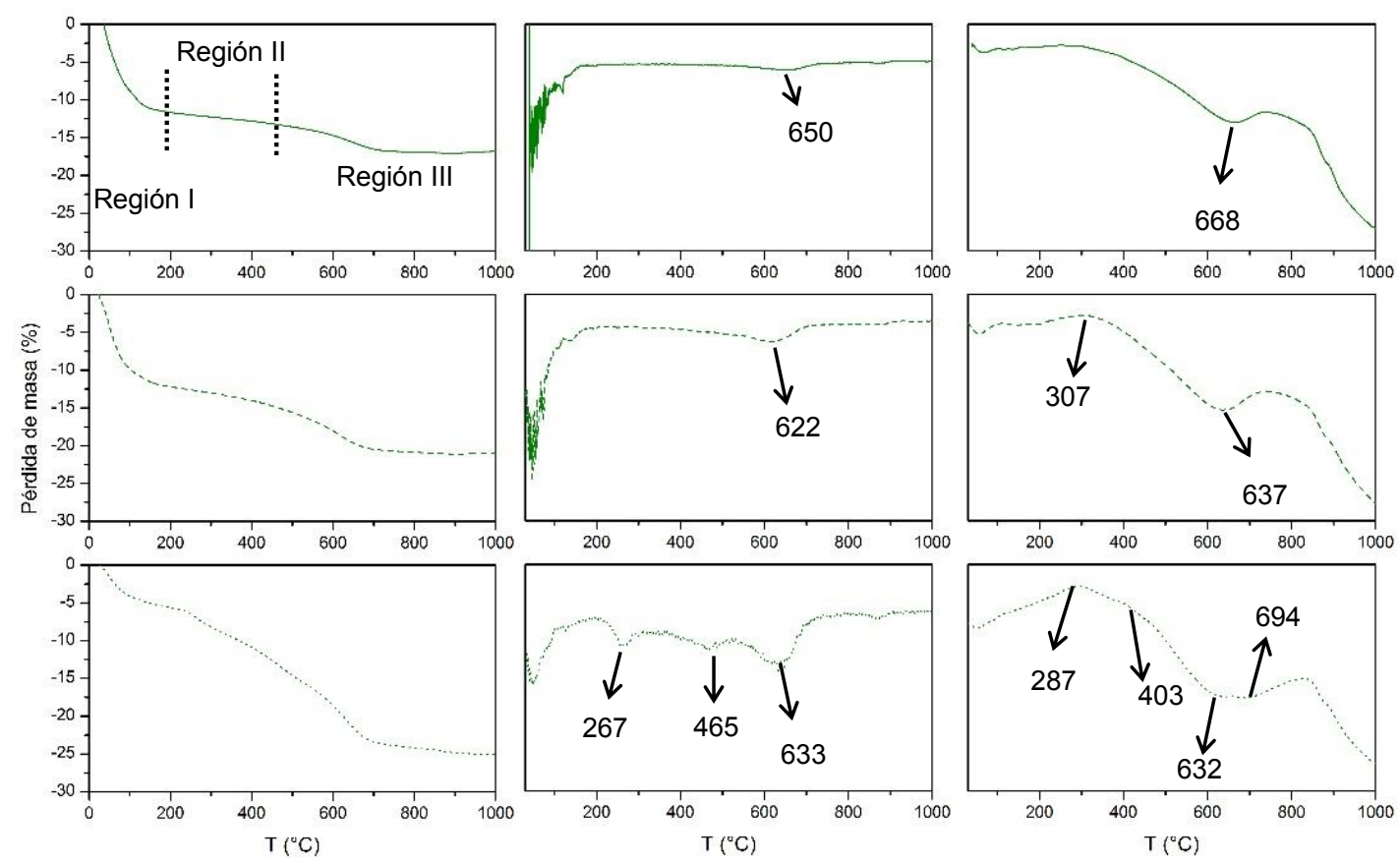

Figura 5.8 Curvas de análisis térmicos. De izquierda a derecha: Tg, DTg y ATD de (-) Mt-Cu, (--) TBZ-Mt$\mathrm{Cu}$ y $(\cdots)$ IMZ-Mt-Cu. 
Los análisis térmicos confirmaron la incorporación del metal y de los fungicidas en el material. En $\mathrm{Mt}-\mathrm{Cu}$, la coordinación del metal con los fungicidas generó una disminución en el contenido de agua por el intercambio de moléculas de agua de la esfera de hidratación del metal por los ligandos TBZ e IMZ. La disminución del contenido de agua fue más marcada en Mt-Cu-IMZ que en Mt-Cu-TBZ debido a que la primera presentó mayor fungicida adsorbido.

Tabla 5.8 Reacciones térmicas, picos DTg y ATD $\left[{ }^{\circ} \mathrm{C}\right]$ y porcentajes de pérdida de masa [\%] de Mt-Cu y productos de adsorción de TBZ e IMZ.

\begin{tabular}{|c|c|c|c|c|c|}
\hline & & & $\mathrm{Mt}-\mathrm{Cu}$ & TBZ-Mt-Cu & $\mathrm{IMZ}-\mathrm{Mt}-\mathrm{Cu}$ \\
\hline \multirow[t]{6}{*}{ Región I } & \multicolumn{2}{|c|}{ Intervalo de $\mathrm{T}$} & $30-200$ & $30-200$ & 20-194 \\
\hline & $\begin{array}{l}\text { Desorción de agua } \\
\text { fisisorbida }\end{array}$ & DTg & n.d. & 45 & 48 \\
\hline & & ATD (endo) & 70 & 57 & 54 \\
\hline & $\begin{array}{l}\text { Deshidratación de cationes } \\
\text { interlaminares }\end{array}$ & & & & 125 \\
\hline & & ATD (endo) & $118 ; 137$ & 142 & n.d. \\
\hline & Pérdida de masa total & $\mathrm{Tg}$ & 11,7 & 11,0 & 5,6 \\
\hline \multirow[t]{7}{*}{ Región II } & \multicolumn{2}{|c|}{ Intervalo de $\mathrm{T}$} & n.c. & $200-500$ & $194-371$ \\
\hline & \multirow[t]{2}{*}{$\begin{array}{l}\text { 1er Paso de oxidación de } \\
\text { contenido orgánico. }\end{array}$} & & n.c. & n.d. & 267 \\
\hline & & ATD (exo) & n.c. & 307 & 287 \\
\hline & \multicolumn{2}{|c|}{ Intervalo de $\mathrm{T}$} & n.c. & n.d. & $371-531$ \\
\hline & $\begin{array}{l}\text { 2do Paso de oxidación de } \\
\text { contenido orgánico. }\end{array}$ & & n.c. & n.d. & 465 \\
\hline & & ATD (exo) & n.c. & n.d. & 403 \\
\hline & Pérdida de masa total & $\mathrm{Tg}$ & 2,0 & 4,5 & 10,7 \\
\hline \multirow[t]{6}{*}{ Región III } & \multicolumn{2}{|c|}{ Intervalo de $\mathrm{T}$} & $500-800$ & $500-800$ & $371-531$ \\
\hline & Deshidroxilación & DTg & 650 & 622 & 633 \\
\hline & & ATD (endo) & 668 & 637 & 632 \\
\hline & $\begin{array}{l}\text { Oxidación de restos } \\
\text { carbónicos }\end{array}$ & & & & \\
\hline & & ATD (exo) & n.c. & n.d. & 694 \\
\hline & Pérdida de masa total & $\mathrm{Tg}$ & 2,9 & 4,2 & 7,8 \\
\hline
\end{tabular}

n.c.: no corresponde; n.d: no determinado

En las Regiones II y III, la presencia de los fungicidas provocó el incremento en el porcentaje de pérdida de masa, mayor en la muestra IMZ-Mt-Cu que en TBZ-Mt-Cu debido a que la primera presentó un mayor $Q$. Además se generaron nuevos picos tanto en 
la curva DTg, como en ATD (exotérmicos) asociados a la combustión de los fungicidas. La temperatura de oxidación del TBZ fue de $307^{\circ} \mathrm{C}$. El IMZ por su parte presentó dos picos DTg a 267 y $465{ }^{\circ} \mathrm{C}$, con sus correspondientes picos exotérmicos en ATD, indicando que el fungicida se descompone en dos etapas. En ambos casos las temperaturas de descomposición de los compuestos orgánicos fueron menores a las observados en los productos de adsorción en Mt (Secciones 3.3.3 y 4.3.3) lo cual se asignó a que el $\mathrm{Cu}^{2+}$ actúa como catalizador en la reacción de combustión de compuestos orgánicos [Yermiyahu et al., 2002; Wen-Chen Tsai y Jiang-Jen Lin, 2010; Nzihou y Stanmore, 2013; Azargohar et al., 2014]. Este tema será discutido con mayor profundidad en el Capítulo 6.

Por último, en la Región III, el incremento en la pérdida de masa; el corrimiento del pico de deshidroxilación hacia menores temperaturas y la aparición de una nueva componente exotérmica en la curva ATD del producto IMZ-Mt-Cu, permitieron terminar de confirmar la interacción de fungicida con el sólido.

\subsection{Conclusiones parciales}

En este capítulo se sintetizó un material por intercambio catiónico de una montmorillonita natural con el catión $\mathrm{Cu}^{2+}$ y se evaluó su capacidad de remoción de TBZ e IMZ, desde el punto de vista cinético y de equilibrio. Los estudios en equilibrio se realizaron tanto con los fungicidas por separado como de forma combinada, simultánea y consecutivamente.

Los resultados mostraron que en la muestra $\mathrm{Mt}-\mathrm{Cu}$, el $\mathrm{Cu}^{2+}$ se localiza en los sitios de carga permanente y de superficie externa en forma de complejo hexa-acuo, aunque también podría haber un porcentaje unido a los bordes, debido a las condiciones utilizadas en la síntesis.

El material mejoró de forma pronunciada la capacidad de adsorción de los fungicidas alcanzando porcentajes de remoción mayores al 98\% (para $C_{i}(\mathrm{TBZ})=15 \mathrm{mg} / \mathrm{L}$ y $C_{i}(\mathrm{IMZ})$ = $40 \mathrm{mg} / \mathrm{L}$ ) luego de $10 \mathrm{~min}$ de contacto, cuando se utilizó la relación sólido/solución $=1$ g/L. Por ese motivo, se evaluó la disminución de la relación sólido/solución que condujo al incremento en la cantidad de TBZ e IMZ adsorbidos por unidad de masa. Los modelos de Elovich y de Pseudo Segundo Orden -que admiten la formación de un enlace químico como paso determinante de la velocidad- fueron los que mejor ajustaron a las cinéticas de adsorción. Estos estudios revelaron que el proceso es más rápido para TBZ que para IMZ. En condiciones de equilibrio, el material presenta mayor capacidad de adsorción de IMZ 
que de TBZ. En los estudios de adsorción múltiple se observó que el sólido es capaz de remover ambos fungicidas en forma simultánea y en forma secuencial.

En base a los datos experimentales, se propone que los sitios de adsorción de los fungicidas sean los cationes $\mathrm{Cu}^{2+}$; el paso determinante de la velocidad la formación de complejos de coordinación; y el mecanismo el esquematizado en la Figura 5.9:

$$
\begin{aligned}
& (\equiv \mathrm{X})_{2} \mathrm{Cu}\left(\mathrm{H}_{2} \mathrm{O}\right)_{6}+n \mathrm{TBZ} \leftrightarrow 2(\equiv \mathrm{X})_{2} \mathrm{Cu}\left(\mathrm{H}_{2} \mathrm{O}\right)_{6-2 n} \mathrm{TBZ}_{n}+2 n \mathrm{H}_{2} \mathrm{O} \\
& (\equiv \mathrm{X})_{2} \mathrm{Cu}\left(\mathrm{H}_{2} \mathrm{O}\right)_{6}+m \mathrm{IMZ} \leftrightarrow 2(\equiv \mathrm{X})_{2} \mathrm{Cu}\left(\mathrm{H}_{2} \mathrm{O}\right)_{6-m} \mathrm{IMZ}_{m}+m \mathrm{H}_{2} \mathrm{O}
\end{aligned}
$$

Figura 5.9 Mecanismo de adsorción de TBZ e IMZ en Mt-Cu.

donde $\equiv \mathrm{X}$ son los sitios de carga permanente superficiales neutralizados por la presencia del complejo $\mathrm{Cu}\left(\mathrm{H}_{2} \mathrm{O}\right)_{6}{ }^{2+}$. Se propone que el mecanismo es una reacción de intercambio de ligando, donde la molécula TBZ, por ser un ligando bidentado puede desplazar a dos moléculas de $\mathrm{H}_{2} \mathrm{O}$, mientras que el ligando IMZ -posiblemente monodentado- sólo a una, con lo cual la capacidad de remoción de Mt-Cu de IMZ es mayor. Para confirmar este mecanismo, en el Capítulo 6 se ahondará en el análisis de estos complejos.

Los resultados de este capítulo permiten proponer la utilización de la montmorillonita en la remoción de efluentes industriales y mineros en una primera instancia, y su posterior uso en el tratamiento de efluentes conteniendo fungicidas. 


\subsection{Introducción y objetivos específicos}

El Capítulo 5 indicó una mejoría importante en la adsorción de los fungicidas cuando se incorporó $\mathrm{Cu}^{2+}$ en la estructura de la montmorillonita. Se propuso que el proceso ocurre vía reacción de intercambio de ligando, donde el TBZ o el IMZ desplazan moléculas de agua de la esfera de coordinación del metal. Sin embargo, la coordinación de los compuestos orgánicos con el centro metálico no fue determinada.

Teniendo en cuenta la menor adsorción, tanto de tiabendazol como de imazalil, en las organo-montmorillonitas evaluadas en relación a la montmorillonita natural, y la ventaja en la posterior separación del producto de adsorción de las organo-montmorillonitas, se decidió en este capítulo estudiar la adsorción de TBZ en una organo-montmorillonita

\footnotetext{
${ }^{10}$ Se agradece a Melisa Olvielli y a todo el grupo de Gustavo Curuchet y Roberto Candal de la Escuela de Ciencia y Tecnología e Instituto de Investigación e Ingeniería Ambiental de la Universidad Nacional de San Martín, por las determinaciones de $\mathrm{Cu}^{2+}$ en las muestras y la caracterización del efluente. Además, a Juan Manuel Lázaro del Instituto de Química y Fisicoquímica Biológicas (Universidad de Buenos Aires CONICET) por las determinaciones de EPR.
} 
intercambiada con $\mathrm{Cu}^{2+}$. Para ello, se recurrió a un método de adsorción secuencial: en un primer paso se adsorbió el cation $\mathrm{Cu}^{2+}$ en una organo-montmorillonita presentada en el Capítulo 3 y en segundo paso se evaluó la adsorción de tiabendazol.

La adsorción de metales pesados en organo-montmorillonitas ha sido estudiada profusamente [Lee y Tiwari, 2012]. En general, se busca intercalar compuestos orgánicos que posean nuevos sitios activos (incrementando los sitios propios de la montmorillonita) y mejorando así la afinidad entre el adsorbente y el adsorbato. Esto se logra empleando moléculas que presenten grupos funcionales que interactúan selectivamente con los cationes metálicos. Por ejemplo, los grupos tiol $(-\mathrm{SH})$, amino $\left(-\mathrm{NH}_{2}\right)$ y carboxilo $(-\mathrm{COOH})$ son agentes quelantes muy eficaces empleados en adsorbentes basados en montmorillonitas, para la remoción de metales pesados como $\mathrm{Hg}^{2+}, \mathrm{Pb}^{2+}, \mathrm{Cd}^{2+}[$ Mercier y Detellier, 1995; Szabo et al., 1998; Sheng et al., 1999; Celis et al., 2000; Lagadic et al., 2001; Tonle et al., 2003]. Stathi et al. [2007] obtuvieron organo-montmorillonitas a partir de distintas moléculas conteniendo diversos grupos funcionales $\left(-\mathrm{NH}_{2},-\mathrm{COOH},-\mathrm{SH} \mathrm{o}-\mathrm{CS}_{2}\right)$ que registraron mayores capacidades de adsorción de los cationes $\mathrm{Cd}^{2+}, \mathrm{Pb}^{2+}$ y $\mathrm{Zn}^{2+}$ que el material de partida. Este aumento de adsorción se atribuyó a (i) el aumento del espaciado interlaminar en el orden de 0,3-0,4 nm tras la intercalación de los compuestos orgánicos, facilitando la posterior incorporación del catión metálico a la zona interlaminar, y (ii) el desarrollo de nuevos sitios de adsorción específicos para los metales, con constantes de afinidad más grandes que las de la montmorillonita sin modificar. En el mismo sentido, Abou-El-Sherbini y Hassanien [2010] evaluaron la remoción de $\mathrm{Cu}^{2+}$ en una organomontmorillonita obtenida a partir de una sal derivada del cetilpiridinio. Ellos concluyeron que la amina se intercala en la intercapa de la montmorillonita, y posteriormente el metal se une a la amina a través de los átomos $\mathrm{O}$ y $\mathrm{N}$ de la forma enol del compuesto. Como resultado, observaron mayores niveles de adsorción respecto de la montmorillonita sin modificar en un amplio intervalo de $\mathrm{pH}$ y a tiempos muy cortos (10 min). Otro ejemplo de la remoción de $\mathrm{Cu}^{2+}$ por organo-montmorillonitas es el trabajo de Balomenou et al. [2008], donde se utilizó como agente de intercalación siloxanos amino-funcionalizados. La eficiencia del material en este caso radicó en la cantidad de grupos amino incorporados en la estructura del adsorbente, disponibles para unirse al metal. Además del incremento en la capacidad de remoción, otra ventaja que presentan las organo-montmorillonitas con agentes quelantes, es su inmovilización en la estructura del sólido por formación de complejos de coordinación estables [Brown et al., 2013]. 
El estudio de la adsorción de metales pesados en organo-montmorillonitas obtenidas con sales de alquilamonio ha sido limitado debido a que las cadenas alquílicas de las aminas cuaternarias no ofrecen sitios específicos para los primeros. Sin embargo, éstas actúan como pilares manteniendo las láminas separadas y facilitando el proceso de intercambio catiónico entre el metal pesado disuelto en la solución y los cationes presentes en la intercapa de la montmorillonita [Ouellet-Plamondon et al., 2012]. Oyanedel-Craver y Smith [2006] estudiaron la adsorción de cuatro metales pesados $\left(\mathrm{Pb}^{2+}, \mathrm{Cd}^{2+}, \mathrm{Zn}^{2+}\right.$ y $\left.\mathrm{Hg}^{2+}\right)$ en hexadeciltrimetilamonio- $\mathrm{y}$ benciltrietilamonio-bentonitas, en función del contenido orgánico. Mostraron que la remoción de $\mathrm{Cd}^{2+}, \mathrm{Pb}^{2+}$ y $\mathrm{Zn}^{2+}$ disminuía con el incremento del contenido orgánico lo que se atribuyó a la ocupación de los sitios de carga permanente por parte del surfactante, disminuyendo así los sitios disponibles para su unión con el catión inorgánico. Sin embargo, las capacidades de adsorción obtenidas permitieron que estos materiales sean propuestos como adsorbente múltiples, tanto de metales pesados como de compuestos orgánicos neutros [Oyanedel-Craver y Smith, 2006].

En ese sentido, existen varias publicaciones que reportan el uso de alquilamoniomontmorillonitas en la remoción simultánea o secuencial de metales pesados y compuestos orgánicos. Por ejemplo, Ma et al. [2016] estudiaron la adsorción en forma secuencial y simultánea de $\mathrm{Cu}^{2+}$ y fenol en hexadeciltrimetilamonio-montmorillonitas. Mostraron que la presencia de un contaminante no afectaba la adsorción del otro (ni en la adsorción secuencial, ni en la simultánea) y sugirieron que los sitios de unión eran diferentes para el compuesto orgánico y para el metal. Por su parte, Jin et al. [2014] estudiaron la posibilidad de utilizar hexadeciltrimetilamonio-montmorillonita en la remoción simultánea del antibiótico amoxicilina y $\mathrm{Cu}^{2+}$ de aguas residuales. Su conclusión indicó que mientras la amoxicilina se unía a la organo-montmorillonita a través de un mecanismo de partición, el $\mathrm{Cu}^{2+}$ lo hacía a través de intercambio catiónico.

Teniendo en cuenta estos antecedentes, sumado a los resultados previamente obtenidos en este trabajo de tesis, en este capítulo se generó un nuevo material mediante la adsorción de $\mathrm{Cu}^{2+}$ en la muestra OMt55 (octadeciltrimetilamonio-montmorillonita intercambiada al 55 \%CIC) para luego evaluar su capacidad de remoción de tiabendazol proveniente de efluentes acuosos. Se eligió esta organo-montmorillonita debido a que hay un $45 \%$ CIC de sitios de carga permanente que permanecen disponibles para la unión del $\mathrm{Cu}^{2+}$. La hipótesis es que el $\mathrm{Cu}^{2+}$ presente en la estructura actuará, en un paso subsiguiente, como sitio activo en la adsorción del fungicida. Por lo tanto, los objetivos específicos de este capítulo son los siguientes: 
(i) obtener una organo-montmorillonita (intercambiada con octadeciltrimetilamonio al $55 \% \mathrm{CIC}$ ) con $\mathrm{Cu}^{2+}$ en su estructura;

(ii) estudiar la adsorción y desorción de tiabendazol en el material obtenido en el punto (i);

(iii) caracterizar los productos de adsorción de tiabendazol en esta esta muestra y en la muestra Mt-Cu (obtenida en el Capítulo 5) mediante técnicas específicas que permitan determinar la presencia de complejos de coordinación;

(iv) identificar sitios activos involucrados en la adsorción del fungicida en todos los casos;

(v) comparar la capacidad de adsorción y desorción de la montmorillonita y organomontmorillonita y sus productos de intercambio con $\mathrm{Cu}^{2+}$ en la remoción de tiabendazol proveniente de un efluente real.

\subsection{Materiales y métodos}

\subsubsection{Materiales}

El adsorbato utilizado fue TBZ grado estándar en solución acuosa (preparada de igual manera que en el Capítulo 4) y TBZ presente en un efluente real. La muestra de efluente se extrajo de la pileta de descarte de una empresa abocada al procesamiento de frutas de pepita en la región del Alto Valle de Río Negro. En dicha pileta se acumulan los descartes tanto del drench como del módulo de lavado, según indica la propia empresa. La muestra se tomó en período de cosecha, temporada durante la cual las concentraciones utilizadas de TECTO ${ }^{\circledR} 50$ SC en el drench fue de $1,7 \mathrm{~cm}^{3} / \mathrm{L}$, y en la línea de empaque fue de $2,5 \mathrm{~cm}^{3} / \mathrm{L}$ (referido por la empresa). Teniendo en cuenta que el TECTO ${ }^{\circledR} 50$ SC se comercializa en forma de suspensión acuosa de concentración de 48,5\% m/v, las concentraciones de TBZ utilizadas en el drench y en la línea de empaque corresponden a 824 y $1210 \mathrm{mg} / \mathrm{L}$, respectivamente. Sin embargo, se espera que en la pileta de decantación la concentración de principio activo disuelta sea apreciablemente menor por varios motivos: los líquidos que circulan en el drench son reutilizados sucesivamente [Colodner, 2011]; las frutan retienen en sus cáscaras y semillas parte de los principios activos que se aplican [Coscollá, 2008]; se desarrollan procesos de descomposición y de precipitación del TBZ al estar en contacto con otras especies químicas y sólidos dispersos, bajo la acción del sol y de microorganismos. La concentración de TBZ fue determinada centrifugando el efluente (para separar los sólidos dispersos) e inyectando en HPLC acoplado a los detectores de UV y FL, según la técnica descripta en la Sección 4.2.3. El promedio de cinco muestras indicó 
que la concentración de TBZ disuelta es $(5,7 \pm 0,3) \mathrm{mg} / \mathrm{L}$. El pH del efluente (sin tratar) fue de $6,7 .^{11}$

Los adsorbentes que se utilizaron fueron $\mathrm{Mt}$, OMt55 y $\mathrm{Mt}-\mathrm{Cu}$ (caracterizados en los Capítulos 3 y 5) y el producto de adsorción de $\mathrm{Cu}^{2+}$ en OMt55 (OMt55-Cu). La última muestra, se obtuvo según lo descripto en la Sección 5.2.1, utilizando una suspensión de 10 $\mathrm{g}$ de OMt55 en $500 \mathrm{~mL}$ de agua destilada y agregando lentamente $\mathrm{CuSO}_{4} \cdot 5 \mathrm{H}_{2} \mathrm{O}$ equivalente al $150 \%$ CIC de Mt. La suspensión se mantuvo en agitación durante toda la noche, alcanzando un $\mathrm{pH}$ final de 4,7. El producto se lavó tres veces con agua destilada y se secó a $80{ }^{\circ} \mathrm{C}$ antes de su utilización. Para determinar el contenido de $\mathrm{Cu}^{2+}$ en OMt55-Cu, $1 \mathrm{~g}$ de muestra fue digerido con $20 \mathrm{~mL}$ de $\mathrm{HNO}_{3}$ concentrado a $60^{\circ} \mathrm{C}$ hasta la evaporación del líquido. Este procedimiento se repitió, luego el sólido fue resuspendido en una solución de $\mathrm{HNO}_{3}: \mathrm{H}_{2} \mathrm{O}$ (1:1) y la suspensión fue filtrada para recuperar el sobrenadante. La concentración de $\mathrm{Cu}^{2+}$ se determinó por absorción atómica usando un equipo SensAA dual GBC Sci, obteniéndose un valor de $0,124 \mathrm{mmol} / \mathrm{g}$.

Por último, se sintetizó un complejo $\mathrm{Cu}(\mathrm{TBZ})_{2} \mathrm{SO}_{4}$ para lo cual a una solución de 1,22 g de TBZ (6 mmol) en $20 \mathrm{~mL}$ de etanol, se agregó una solución de 0,75 g (3 mmol) de $\mathrm{CuSO}_{4} \cdot 5 \mathrm{H}_{2} \mathrm{O}$ en $5 \mathrm{~mL}$ de agua. Luego de tres días de agitación a temperatura ambiente, un precipitado verde se separó mediante filtración y recristalizó para su purificación [Ennajih et al., 2012]. Finalmente se secó a $60^{\circ} \mathrm{C}$ por 24 h y conservó en desecador hasta su uso. Tanto los resultados de DRX como de análisis térmicos se pudo comprobar que el material sintetizado correspondió al complejo buscado. Estos datos están disponibles en la Figura A2, en el Anexo 1. La muestra se denominó Cu-TBZ.

\subsubsection{Estudios de adsorción-desorción}

\subsubsection{Estudios de adsorción de TBZ grado estándar}

Las isotermas de adsorción de TBZ grado estándar en la muestra Mt-Cu a tres distintas relaciones sólido/solución $(1 ; 0,5$ y $0,1 \mathrm{~g} / \mathrm{L})$ y en Mt a relación sólido/solución $=1 \mathrm{~g} / \mathrm{L}$ fueron presentadas en el Capítulo 5; mientras que la isoterma de adsorción de TBZ en OMt55 a relación sólido/solución $=1 \mathrm{~g} / \mathrm{L}$ se introdujo en el Capítulo 4 . Para completar el

\footnotetext{
${ }^{11}$ Este efluente fue caracterizado por el grupo de investigación de Gustavo Curuchet y Roberto Candal, de la Escuela de Ciencia y Tecnología e Instituto de Investigación e Ingeniería Ambiental, de la Universidad Nacional de San Martín, Buenos Aires, determinándose los siguientes valores: Conductividad = 2,44 mS; DQO $=2020 \mathrm{mg} / \mathrm{L} ;$ sólidos totales $=4,0 \mathrm{~g} / \mathrm{L}$; sólidos volátiles $=2,3 \mathrm{~g} / \mathrm{L}$. Se les agradece por la disponibilidad de los datos.
} 
análisis, en este capítulo se muestran las isotermas de TBZ en Mt y OMt55 a relación sólido/solución $=0,5$ y 0,1 $\mathrm{g} / \mathrm{L} ;$ y en OMt55-Cu a relación sólido/solución $=1 ; 0,5$ y 0,1 g/L. Se usaron tubos de plástico y un tiempo de contacto de 24 h a $25{ }^{\circ} \mathrm{C}$ bajo agitación constante. El intervalo de concentraciones de TBZ utilizado fue de 0,5 a $25 \mathrm{mg} / \mathrm{L}$. Todas las soluciones fueron preparadas en agua. Transcurrido el tiempo de contacto, se centrifugaron las suspensiones a $14.000 \mathrm{rpm}$ durante $15 \mathrm{~min}$. La concentración de TBZ se determinó mediante cromatografía líquida de alta resolución (HPLC) acoplado con un detector UV-visible usando el equipo y las condiciones previamente mencionadas (Sección 4.2.3). A partir de los datos de $Q$ obtenidos de los datos experimentales para $C_{i}(\mathrm{TBZ})=25$ $\mathrm{mg} / \mathrm{L}$, se calculó la eficiencia de la remoción para todas las relaciones sólido/solución empleando la Ecuación 3.5.

Al igual que en los capítulo previos, el $\mathrm{pH}$ de las suspensiones se dejó evolucionar naturalmente, siendo 4,6 (constante, durante todo el tiempo de equilibrio) para la isoterma de adsorción de TBZ en OMt55-Cu y relación sólido/solución $=1 \mathrm{~g} / \mathrm{L}$. El sólido correspondiente al punto de máxima concentración de TBZ en dicha isoterma, luego de separarlo de la suspensión mediante centrifugación, se lavó con agua destilada, se secó en estufa a $60{ }^{\circ} \mathrm{C}$ y se conservó para posteriores caracterizaciones. Este producto se denominó TBZ-OMt55-Cu.

Teniendo en cuenta que en los Capítulos 4 y 5 el modelo que mejor ajustó a las isotermas de adsorción fue el LF, en este capítulo se utilizó únicamente este modelo para ajustar a los datos experimentales con el objetivo de poder comparar los parámetros obtenidos entre los distintos adsorbentes.

\subsubsection{Estudios de adsorción-desorción de TBZ comercial en un efluente real}

Debido a que el efluente real consiste en una suspensión, los ensayos de adsorción se realizaron empleando el efluente sin tratar (E) y el efluente centrifugado (EC). Se evaluaron distintas relaciones sólido/solución (1 y 0,1 g/L); un tiempo de contacto de $24 \mathrm{~h}$ con agitación constante a $25^{\circ} \mathrm{C}$. Las suspensiones, luego de transcurrido el tiempo de contacto, fueron centrifugadas a $14000 \mathrm{rpm}$ y el TBZ del sobrenadante fue determinado por HPLC (Sección 4.2.3). Los resultados se expresan como eficiencias de remoción (E, Ecuación 3.6) y el valor es el promedio de cinco ensayos.

Tras el ensayo de adsorción de TBZ, las suspensiones EC con relación sólido/solución $=1 \mathrm{~g} / \mathrm{L}$, se sometieron a un proceso de centrifugado, aislado, secado y se conservaron para realizar los experimentos de desorción y lixiviación. Las muestras se denominaron anteponiendo EC- al nombre de los adsorbentes. 
Teniendo en cuenta que en las condiciones de desorción evaluadas en el Capítulo 4 (agua, relación sólido/solución $=1 \mathrm{~g} / \mathrm{L}$ ) no se encontró desorción de TBZ de los adsorbentes Mt y OMt55, en este capítulo se decidió ensayar otras condiciones de desorción, con el objetivo de evaluar la posibilidad de desorber el TBZ en un volumen menor de líquido. Para ello los experimentos consistieron en el agregado de solución $[\mathrm{NaOH}]=0,1 \mathrm{M} \mathrm{o}[\mathrm{HCl}]=0,1 \mathrm{M}$ a los productos de adsorción del efluente centrifugado en una relación sólido/solución $=4 \mathrm{~g} / \mathrm{L}$, seguido de agitación durante toda la noche, centrifugado y análisis del sobrenadante por HPLC.

\subsubsection{Estudios de lixiviación}

Con el objetivo de evaluar la movilidad de TBZ y $\mathrm{Cu}^{2+}$ en los sólidos producidos tras la adsorción del metal y el fungicida (tanto proveniente de la solución estándar como del efluente real centrifugado) se ensayó, para cada sistema, el Método 1312 de la Agencia de Protección Ambiental de Estados Unidos [EPA, 1990]. Para ello, se formó una suspensión con los sólidos conteniendo $\mathrm{Cu}^{2+}$ y/o TBZ y una solución diluida de $\mathrm{H}_{2} \mathrm{SO}_{4} / \mathrm{HNO}_{3}(60 / 40$ $\mathrm{p} / \mathrm{p}$ ) a $\mathrm{pH}=4,2$. La relación sólido/solución utilizada fue $0,05 \mathrm{~g} / \mathrm{L}$. Esta suspensión se mantuvo en agitación durante toda la noche a $25^{\circ} \mathrm{C}$, luego se centrifugó y el $\mathrm{Cu}^{2+} \mathrm{y} / \mathrm{o}$ el TBZ se cuantificaron mediante absorción atómica y HPLC, respectivamente. Este procedimiento se repitió dos veces en cada muestra. Los valores obtenidos son valores promedios y se expresan como porcentajes respecto del contenido inicial de fungicida y de metal.

\subsubsection{Métodos de caracterización}

En este capítulo se muestran las caracterizaciones del adsorbente OMt55-Cu y el producto de adsorción TBZ-OMt55-Cu mediante DRX, potencial zeta y análisis térmicos que se desarrollaron en las condiciones indicadas en la Sección 3.2.2. Se midió el diámetro aparente (DAp) de las muestras Mt-Cu y OMt55-Cu en las condiciones descriptas en dicha sección. Además, las muestras $\mathrm{Mt}-\mathrm{Cu}, \mathrm{OMt} 55-\mathrm{Cu}$, sus productos de adsorción y el complejo de coordinación sintetizado $\mathrm{Cu}(\mathrm{TBZ})_{2}\left(\mathrm{SO}_{4}\right)$ fueron caracterizadas mediante resonancia paramagnética electrónica (EPR).

\subsubsection{Resonancia paramagnética electrónica (EPR)}

La resonancia del espín electrónico o espectroscopía de resonancia paramagnética electrónica (EPR, por sus siglas en inglés) consiste en inducir transiciones entre los subniveles Zeeman electrónicos de un sistema paramagnético (ion, radical, electrón libre, 
etc.) aplicando un campo electromagnético de frecuencia (normalmente en el rango de las microondas), polarización e intensidad adecuados.

En presencia de un campo magnético externo, $H$, la energía electrónica es

$$
E\left(m_{s}\right)=E_{o}+g \cdot \beta \cdot H \cdot m_{s}
$$

(Ecuación 6.1)

donde $E_{o}$ es la energía en ausencia del campo magnético; $g$ (constante adimensional) es el factor $g$ electrónico cuyo valor para un electrón libre es 2.002319; $\beta$ es el magnetón de Bohr y vale 9,274.10-24 $\mathrm{J} / \mathrm{T} ; H$ es el campo magnético en Gauss y $m_{s}$ es el número cuántico magnético, cuyo valor es $\pm 1 / 2$.

En un experimento de EPR las transiciones son inducidas entre dos estados Zeeman $\left(m_{s}\right.$ $=+1 / 2$ y $\left.m_{s}=-1 / 2\right)$ generados en un fuerte campo magnético, por medio de una radiación de microondas de frecuencia $v_{\mathrm{o}}$ determinada por la condición de resonancia:

$$
\Delta E=E\left(m_{s}=+1 / 2\right)-E\left(m_{s}=-1 / 2\right)=h v_{o}
$$

y sustituyendo en la Ecuación 6.1, resulta:

$$
h v_{o}=g \cdot \beta \cdot H
$$

(Ecuación 6.3)

Es decir que la situación de resonancia puede alcanzarse manteniendo constante el valor del campo magnético y variando la frecuencia de los fotones o viceversa, lo que cambia la separación de los niveles, según se muestra en la Figura 6.1.

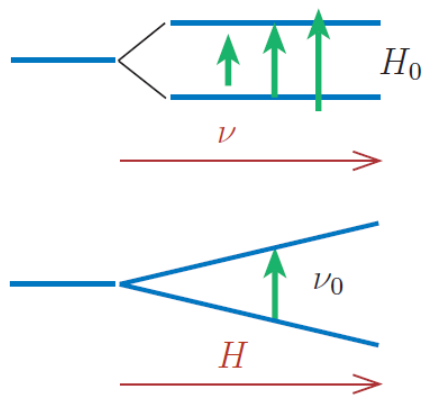

(a)

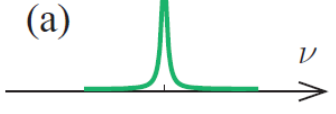

(b)

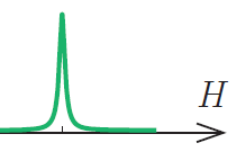

Figura 6.1 Maneras de obtener el espectro de resonancia magnética (a) barriendo la frecuencia de fotones y (b) barriendo el campo magnético. Fuente: [López, Espectroscopía de Resonancia Magnética].

Por razones experimentales, los equipos EPR convencionales trabajan a frecuencia fija y campo magnético variable. Se observa en la Figura 6.1 que la separación entre los niveles de energía es una función lineal del campo magnético aplicado (Ecuación 6.3). Al ir variando $H$ cuando se cumpla la Ecuación 6.3 se tendrá absorción por parte de la muestra, y el campo magnético correspondiente a la absorción se denomina campo de resonancia, $H_{\text {, }}$. 
En general debido al sistema de detección empleado en EPR, el espectro observado en el monitor corresponde a la primera derivada del espectro de absorción.

Una condición necesaria para que una muestra presente EPR es que contenga al menos un átomo con sus electrones desapareados (por ejemplo, radicales libres, iones de metales de transición, etc.). $\mathrm{El}$ ion $\mathrm{Cu}^{2+}$ cumple con esta condición, ya que es un metal con configuración d $\mathrm{d}^{9}$ (paramagnético). Ya que el espectro EPR de un complejo de coordinación puede brindar información valiosa con respecto a estados de oxidación del metal, tipo de ligando y simetría, se lo utilizó en este capítulo para caracterizar a las muestras intercambiadas con $\mathrm{Cu}^{2+}$ y determinar su complejación con el TBZ.

La información que aporta un espectro EPR específicamente para el metal en cuestión, es la siguiente:

(i) la señal permite discernir el estado de oxidación del metal en las muestras, ya que de sus dos formas estables $\left(\mathrm{Cu}^{2+} \mathrm{y} \mathrm{Cu}^{+}\right)$sólo la primera presenta señal en EPR (la segunda es diamagnética);

(ii) el valor del factor de desdoblamiento espectroscópico $(g)$ da información sobre la geometría del complejo, adquiriendo diferentes valores según su alineación

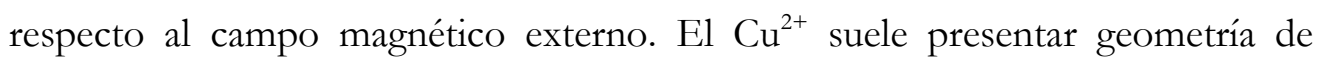
octaedro elongado por efecto Jahn-Teller. En este caso, cuando el eje ₹ se alinea con el campo magnético externo, el valor de $g$ se conoce como $g_{I I}$ o $g_{\vartheta}$ mientras que a los valores de $g$ en los ejes $x$ e $y$ se los conoce como $g_{x}$ y $g_{y}$ o $g_{\perp}$. En este entorno octaédrico distorsionado $g_{I I}>g_{\perp}>2,00$ ya que los cuatro ligandos dispuestos aproximadamente en un plano (que incluye al ion metálico) presentarán una mayor interacción, mientras que los otros dos ligandos dispuestos en una línea recta perpendicular al plano (disposición axial) estarán más alejados y más débilmente unidos al centro metálico, presentando una influencia menor sobre las propiedades magnéticas y ópticas del complejo. A su vez el valor de $g_{I I}$ está relacionado con el grado de covalencia en la interacción metal-ligando, de modo que generalmente se cumple que en un entorno iónico $g_{I I}>2,3$, mientras que en un entorno covalente, $g_{I I}<2,3$;

(iii) la constante de acoplamiento hiperfina $(A)$ originada en la interacción del electrón desapareado con su propio núcleo (si $I \neq 0$ ) brinda información sobre la identidad del núcleo del ion o átomo. Se manifiesta como una estructura hiperfina originada en el desdoblamiento de niveles energéticos. Para el caso de un complejo de $\mathrm{Cu}^{2+}(\mathrm{I}=3 / 2)$ con elongación tetragonal, de manera similar a lo 
que ocurre con los valores de $g$, la interacción hiperfina es anisotrópica $\left(A_{x}=A_{y}\right.$ $\left.\neq A_{2}\right)$.

Los experimentos EPR de las muestras Mt-Cu, TBZ-Mt-Cu, OMt55-Cu, TBZ-OMt55$\mathrm{Cu}$ y $\mathrm{Cu}-\mathrm{TBZ}$ fueron realizados con un Espectrómetro Bruker EMX Plus a $20{ }^{\circ} \mathrm{C}$. Las condiciones del ensayo fueron las siguientes: frecuencia de microondas de 9,87 GHz; campo de modulación de $100 \mathrm{kHz}$; amplitud de la modulación de $4 \mathrm{G}$; potencia de microondas de 0,2 mW; resolución de 2040 puntos. El número de escaneos en todos los experimentos fue 100 .

\subsection{Resultados y discusión}

\subsubsection{Estudios de adsorción}

\subsubsection{TBZ grado estándar}

La Figura 6.2 muestra las isotermas de adsorción de TBZ en las distintas relaciones sólido/solución evaluadas. Nótese que con el objetivo de su comparación se introdujeron aquí las isotermas de los Capítulos 3 y 4. Los resultados del ajuste de LF a los datos experimentales se enumeran en la Tabla 6.1.
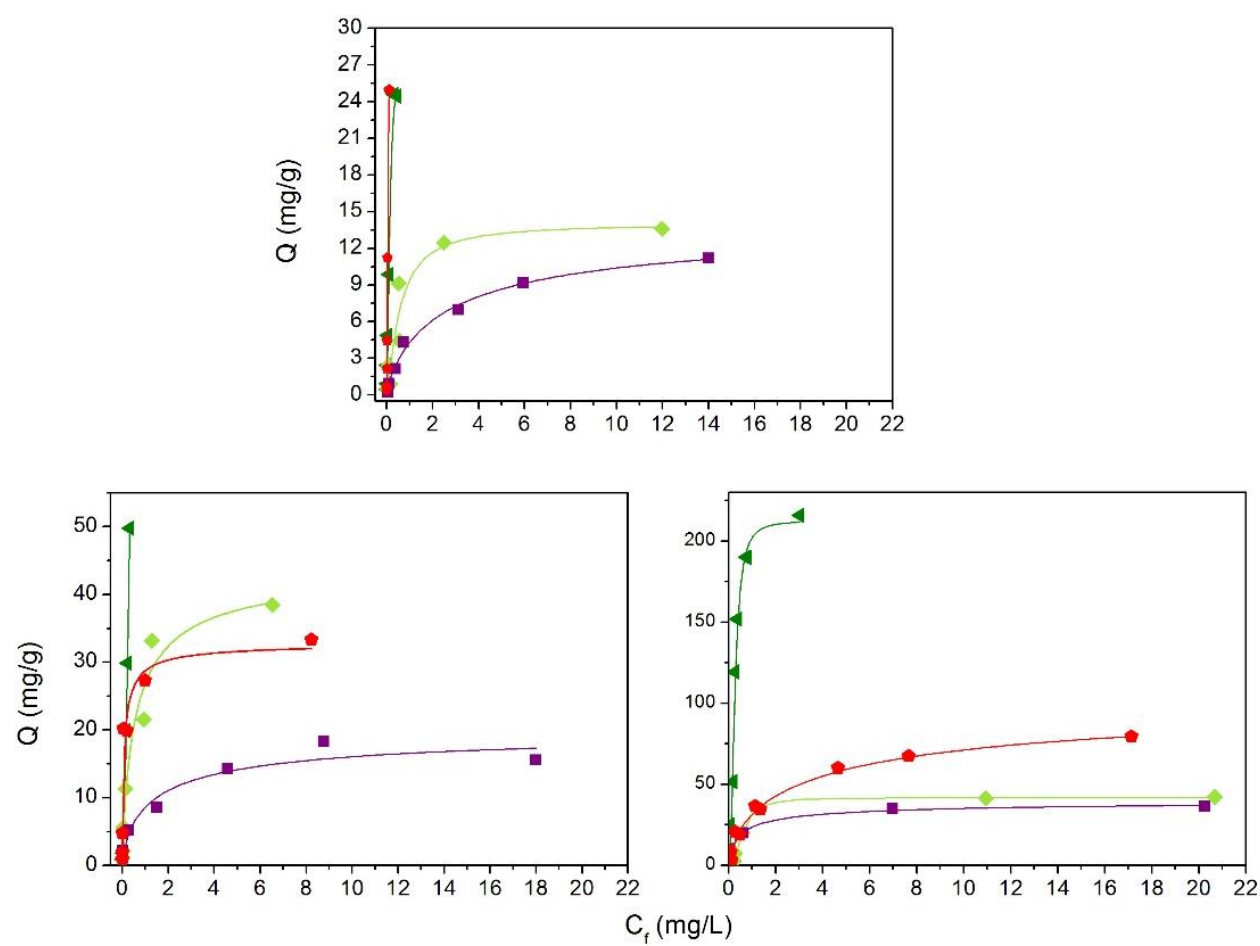

Figura 6.2 Isotermas de adsorción de TBZ en (») Mt, (४) Mt-Cu, (๘) OMt55 y ( $)$ OMt55-Cu. Relación sólido/solución = $1 \mathrm{~g} / \mathrm{L}$ (arriba); 0,5 g/L (abajo, izquierda) y 0,1 g/L (abajo, derecha). Las líneas sólidas corresponden al ajuste de Langmuir-Freundlich. 
Los resultados en todas las condiciones coinciden con lo observado a lo largo de esta tesis. En todos los casos, la muestra OMt55 mostró una curva de adsorción por debajo de la de Mt, confirmando que la incorporación de ODTMA, en lugar de generar nuevos sitios de adsorción para el fungicida, ocupa los sitios de carga permanente de la capa siloxánica y disminuye los sitios disponibles para la unión del TBZ. Esto es más evidente cuando se observa, en la Tabla 6.1, que el valor de $Q_{\max , S}$ y $K_{S}$ de la muestra OMt55 permanecen iguales o disminuyen respecto a los respectivos valores de Mt, cualquiera sea la relación sólido/solución. Por su parte, la incorporación de $\mathrm{Cu}^{2+}$ en $\mathrm{OMt} 55$ generó un incremento notorio en la cantidad adsorbida de TBZ, que se tradujo también en un aumento del valor de $Q_{\text {max }, S}$ en la muestra OMt55-Cu respecto a OMt55, en todas las condiciones (Tabla 6.1).

Tabla 6.1 Parámetros obtenidos mediante el ajuste del modelo Langmuir-Freundlich a las isotermas de adsorción de TBZ.

\begin{tabular}{|c|c|c|c|c|c|}
\hline $\begin{array}{c}\text { Relación } \\
\text { sólido/solución }\end{array}$ & Muestra & $\mathrm{R}^{2}$ & $Q_{\max , S}[\mathrm{mg} / \mathrm{g}]$ & $K_{s}[\mathrm{~L} / \mathrm{mg}]$ & $1 / n$ \\
\hline \multirow{4}{*}{$1 \mathrm{~g} / \mathrm{L}$} & Mt & 0,920 & $14 \pm 2$ & $1,7 \pm 0,8$ & $1,2 \pm 0,7$ \\
\hline & $\mathrm{Mt}-\mathrm{Cu}$ & 0,993 & $28 \pm 2$ & $8 \pm 1$ & $1,7 \pm 0,3$ \\
\hline & OMt55 & 0,989 & $14 \pm 2$ & $0,3 \pm 0,1$ & $0,8 \pm 0,1$ \\
\hline & $\mathrm{OMt} 55-\mathrm{Cu}$ & 0,970 & $27 \pm 2$ & $13 \pm 2$ & $1,7 \pm 0,4$ \\
\hline \multirow{4}{*}{$0,5 \mathrm{~g} / \mathrm{L}$} & Mt & 0,967 & $44 \pm 8$ & $2,0 \pm 0,5$ & $0,8 \pm 0,2$ \\
\hline & $\mathrm{Mt}-\mathrm{Cu}$ & 0,997 & $(15 \pm 8) \cdot 10^{1}$ & $2,2 \pm 0,1$ & $1,8 \pm 0,3$ \\
\hline & OMt55 & 0,928 & $21 \pm 6$ & $0,6 \pm 0,1$ & $0,7 \pm 0,3$ \\
\hline & OMt55-Cu & 0,952 & $33 \pm 3$ & $13 \pm 6$ & $0,7 \pm 0,2$ \\
\hline \multirow{4}{*}{$0,1 \mathrm{~g} / \mathrm{L}$} & Mt & 0,995 & $42 \pm 1$ & $1,6 \pm 0,1$ & $2,2 \pm 0,4$ \\
\hline & $\mathrm{Mt}-\mathrm{Cu}$ & 0,982 & $(21 \pm 1) \cdot 10^{1}$ & $3,7 \pm 0,3$ & $2,2 \pm 0,5$ \\
\hline & OMt55 & 0,993 & $41 \pm 4$ & $1,6 \pm 0,8$ & $0,6 \pm 0,1$ \\
\hline & $\mathrm{OMt} 55-\mathrm{Cu}$ & 0,983 & $(11 \pm 2) \cdot 10^{1}$ & $0,3 \pm 0,1$ & $0,7 \pm 0,1$ \\
\hline
\end{tabular}


Las muestras Mt-Cu y OMt55-Cu presentaron eficiencias de remoción (E) mayores al 95\% cuando la relación sólido/solución fue $1 \mathrm{~g} / \mathrm{L}$ (Figura 6.3), cercano al doble de la eficiencia de las muestras Mt y OMt55. Este buen desempeño de los adsorbentes fue lo que motivó a disminuir la relación sólido/solución, de manera de conocer el límite inferior de sólido que debería utilizarse para eliminar el TBZ presente en una solución acuosa. Al bajar el contenido de adsorbente por unidad de volumen a la mitad $(0,5 \mathrm{~g} / \mathrm{L})$, la eficiencia se mantuvo constante para $\mathrm{Mt}-\mathrm{Cu}$, mientras que disminuyó del 96 al 68\% para OMt55-Cu. Este comportamiento podría relacionarse -admitiendo que los sitios de unión de TBZ en esta muestra son los iones $\mathrm{Cu}^{2+}$ - con el contenido de metal en cada muestra. Una diminución mayor de la relación sólido/solución (a $0,1 \mathrm{~g} / \mathrm{L}$ ), promovió una caída en la eficiencia de OMt55-Cu al 32\%, mientras que la muestra Mt-Cu removió el 86\% del TBZ disuelto inicialmente. Nuevamente, estos resultados podrían relacionarse con el contenido de $\mathrm{Cu}^{2+}$ en las muestras aunque es llamativo que la caída de la eficiencia sea tanto más para OMt55-Cu que para Mt-Cu. De la misma forma, hay un aumento desigual de $Q$ en las isotermas de adsorción al disminuir la relación sólido/solución. En el caso extremo, para la relación sólido/solución más baja $(0,1 \mathrm{~g} / \mathrm{L})$, la muestra Mt-Cu presentó una Q que, para el intervalo de $C_{f}$ entre 1,5 y $2 \mathrm{mg} / \mathrm{L}$, superó en un orden de magnitud el $Q$ de las muestras Mt, OMt55 y OM55-Cu (Figura 6.2). Es decir, la disminución de la relación sólido/solución llevó a disminuir la eficiencia de remoción (E, Figura 6.3) y a aumentar la cantidad adsorbida por unidad de masa de adsorbente (Q, Figura 6.2, Tabla 6.1) en proporciones que fueron diferentes para cada muestra.

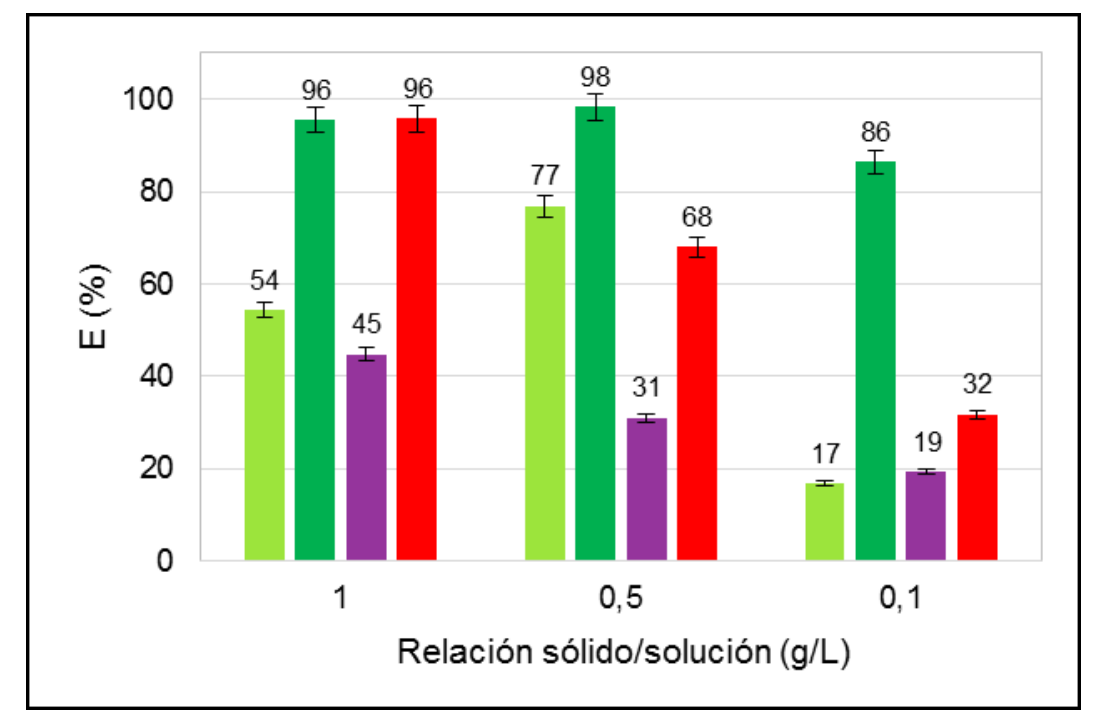

Figura 6.3 Eficiencia de remoción de TBZ $\left(C_{i}=25 \mathrm{mg} / \mathrm{L}\right)$ de ( $(\varpi) \mathrm{Mt},(\varpi) \mathrm{Mt}-\mathrm{Cu},(\varpi)$ OMt55 y (匹) OMt55-Cu. 
El fenómeno de aumento de $E$ y disminución de $Q$ al incrementar la dosis de sólido, aunque parece paradójico, puede explicarse al menos por dos efectos.

El primero tiene que ver con lo mencionado en la Sección 5.3.1 respecto a los sitios que permanecen insaturados a dosis altas de adsorbente. El incremento de la relación sólido/solución implica un aumento de sitios activos en el mismo volumen de solución. Esto provoca que una mayor cantidad de adsorbato se una a la superficie del sólido y se traduce en un incremento en el porcentaje de adsorbato removido de la solución (mayor E). Al mismo tiempo, si la cantidad de sólido es muy elevada respecto al adsorbato, un exceso de sitios activos permanecen insaturados llevando a valores más bajos de cantidad adsorbida por unidad de masa (menor Q) [Akpomie y Dawodu, 2014].

El segundo efecto está asociado a fenómenos físicos, interacciones intra-particulares que se tornan más importantes en cuanto la cantidad de sólido por unidad de volumen aumenta. Se tratan de interacciones electrostáticas que generan la agregación de las partículas, bloqueando sitios activos y disminuyendo el área efectiva disponible para el adsorbato. Además, estas interacciones electrostáticas modifican las cargas superficiales del sólido, lo cual puede a su vez modificar las energías de interacción entre el sólido y el adsorbato [Malamis y Katsou, 2013]. Por lo tanto, aunque el número de sitios activos por unidad de masa de un adsorbente no dependa de la masa de éste, el aumento de la cantidad de sólido en un volumen de líquido dado reduce el número de sitios activos por unidad de masa, ya que la superficie efectiva para la interacción con el adsorbato disminuye [Gupta y Bhattacharyya, 2006].

Conjuntamente con la cantidad de $\mathrm{Cu}^{2+}$ presente en la suspensión, posiblemente también la ubicación del metal en la estructura del adsorbente y los fenómenos interparticulares, determinen que el ion esté o no disponible para interactuar con el fungicida en la interfase sólido/solución y, por lo tanto, el valor que adquieren $Q$ y E. Los efectos de interacción inter-particulares que se nombraban más arriba no son los mismos en la organo-montmorillonita que en la montmorillonita natural, ya que las cargas superficiales son diferentes, su interacción con el solvente y consecuentemente su capacidad de agregación también lo son (Sección 3.3). De hecho, el diámetro aparente de las muestras Mt-Cu y OMt55-Cu fueron $772 \pm 50$ y $2015 \pm 75$ nm, respectivamente. El tamaño distinto de las partículas en la interfase sólido/solución es esperable que afecte de manera diferente el potencial químico en la superficie de los materiales y por lo tanto su interacción con el compuesto orgánico (TBZ) y que ello explique las diferencias entre las muestras Mt-Cu y OMt55-Cu. 


\subsubsection{Estudios de adsorción-desorción de TBZ comercial en un efluente real}

La Figura 6.4 muestra los resultados de eficiencia de remoción de TBZ presente en el efluente real $\left(C_{i}(\mathrm{TBZ})=5,7 \pm 0,3 \mathrm{mg} / \mathrm{L}\right)$ por parte de los distintos adsorbentes, que presentaron la misma tendencia analizada en la sección anterior. Puede notarse que el paso de centrifugación previo mejora los porcentajes de remoción, probablemente porque el sólido disperso en el efluente real contiene tiabendazol adsorbido. Es importante tener en cuenta que el efluente, además de contener TBZ, posiblemente contenga restos de detergentes y otros fungicidas utilizados tanto en la línea de empaque como en el drench, lo que explicaría los cambios en los porcentajes de remoción que fueron positivos en algunos casos (por ejemplo la Mt mejoró su desempeño) y negativos en otros (la OMt55-Cu empeoró, sobre todo a relación sólido/solución $=0,1 \mathrm{~g} / \mathrm{L}$ ). Sin embargo, la muestra Mt-Cu presentó un excelente rendimiento, independientemente de que el efluente sea centrifugado o no, cuando se utilizó la relación sólido $/$ solución $=1 \mathrm{~g} / \mathrm{L}$.

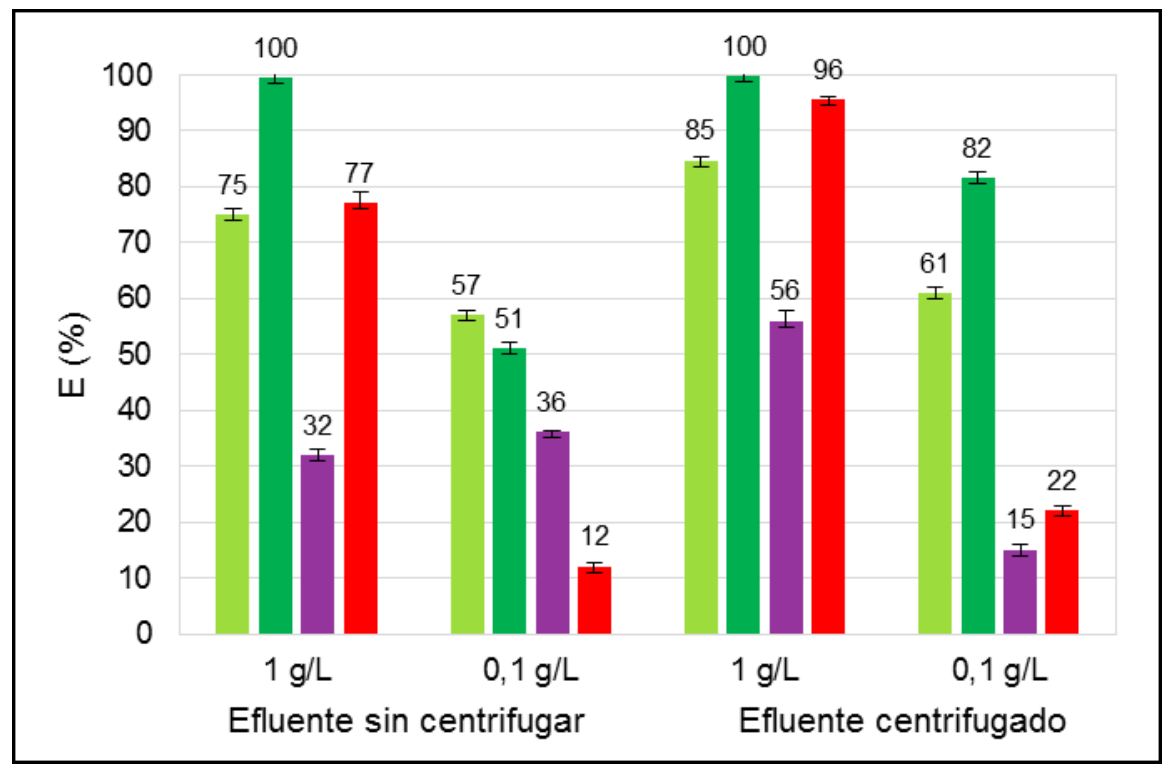

Figura 6.4 Eficiencia de remoción de TBZ $\left(C_{i}=5,7 \pm 0,3 \mathrm{mg} / \mathrm{L}\right)$ proveniente de un efluente real

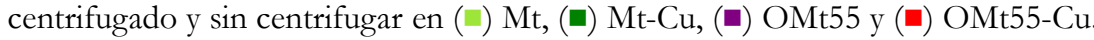

La Tabla 6.2 muestra los resultados de los ensayos de desorción de los productos de adsorción con el efluente centrifugado. El medio más efectivo para la desorción fue la utilización de $\mathrm{NaOH}$, posiblemente por dos efectos sinérgicos: el agregado de $\mathrm{OH}^{-}$ deprotona el $\mathrm{TBZH}^{+}$unido a la capa siloxánica de la Mt y de la OMt55, y el compuesto en su forma neutra interactúa con la superficie siloxánica con una energía de interacción 233 $\mathrm{kcal} / \mathrm{mol}$ menos intensa (Capítulo 4); por otro lado, el agregado de $\mathrm{Na}^{+}$a concentraciones elevadas respecto al $\mathrm{TBZH}^{+}$adsorbido, compite con éste por los sitios de carga 
permanente. Ambos procesos conducirían a la liberación de TBZ al medio acuoso en las muestras Mt y OMt55. Nótese que el agregado de ácido prácticamente no desorbe TBZ. Esto es coincidente con la dependencia de $Q$ con el $\mathrm{pH}$ estudiado y reportado en la bibliografía, donde se encontró que el TBZ es adsorbido a porcentajes de remoción elevados preferentemente en medios ácidos y neutros [Aharonson y Kafkafi, 1975b; Lombardi et al., 2003; 2006].

Tabla 6.2 Cantidad adsorbida $(Q)$ y porcentajes de desorción de TBZ en distintas condiciones.

\begin{tabular}{|c|c|c|c|}
\hline Muestra & $\mathcal{Q}[\mathrm{mg} / \mathrm{g}]$ & Desorción en $\mathrm{HCl}(0,1 \mathrm{M})[\%]$ & Desorción en $\mathrm{NaOH}(0,1 \mathrm{M})[\%]$ \\
\hline EC-Mt & $4,84 \pm 0,05$ & $0,12 \pm 0,05$ & $17 \pm 0,3$ \\
EC-Mt-Cu & $5,7 \pm 0,3$ & $0,004 \pm 0,001$ & $0,004 \pm 0,006$ \\
EC-OMt55 & $3,2 \pm 0,1$ & $0,22 \pm 0,03$ & $20 \pm 0,7$ \\
EC-OMt55-Cu & $5,47 \pm 0,03$ & $0,09 \pm 0,02$ & $0,31 \pm 0,06$ \\
\hline
\end{tabular}

La presencia de $\mathrm{Cu}^{2+}$ en la estructura de los materiales conllevó a una disminución importante en los porcentajes de desorción de TBZ, en más de un orden de magnitud en todas las muestras y condiciones, probablemente debido a que los sitios y fuerzas de interacción entre el TBZ y los materiales difieren en presencia de $\mathrm{Cu}^{2+}$ en la matriz. Esto será profundizado en la próxima sección.

\subsubsection{Estudios de lixiviación}

Con el objetivo de estudiar el efecto ambiental adverso de los sólidos generados, en vistas de su posible aplicación en el tratamiento de efluentes de la producción frutícola, se realizaron experimentos de lixiviación en muestras seleccionadas, cuyos resultados se enumeran en la Tabla 6.3. Las lixiviaciones, tanto del ion metálico como del fungicida, se expresaron como un porcentaje respecto del contenido inicial de dicha especie en el sólido. Es por ello que en la Tabla 6.3 se incluyeron también la cantidad de fungicida adsorbido y de $\mathrm{Cu}^{2+}$ por unidad de masa.

La Tabla 6.3 muestra que el porcentaje de metal lixiviado disminuyó del 24 al 9,7\% en $\mathrm{Mt}-\mathrm{Cu}$ y $\mathrm{OMt}-\mathrm{Cu}$, respectivamente. Esta disminución fue asignada al impedimento estérico que experimenta el $\mathrm{Cu}^{2+}$ presente en los sitios interlaminares de la organo-montmorillonita para difundir hacia la solución por la presencia de las cadenas alquílicas de la amina.

La presencia de TBZ en las muestras (ya sea en grado estándar o proveniente de un efluente real) llevó a una disminución aún más pronunciada del porcentaje de lixiviado del 
metal, de 24\% en Mt-Cu a 0,9 y 0,7\% en TBZ-Mt-Cu y E-Mt-Cu, respectivamente; y de 9,7\% en OMt55-Cu a 2,4 y 3,1\% en TBZ-OMt55-Cu y E-OMt55-Cu, respectivamente.

Tabla 6.3 Contenido de $\mathrm{Cu}^{2+}$, cantidad adsorbida de TBZ por unidad de masa $(Q)$ y porcentajes de lixiviación del metal y del TBZ.

\begin{tabular}{|c|c|c|c|c|}
\hline Muestra & $\begin{array}{c}\text { Contenido de } \mathrm{Cu}^{2+} \\
{[\mathrm{mmol} / \mathrm{g}]}\end{array}$ & $\mathcal{L}[\mathrm{mg} / \mathrm{g}]$ & $\mathrm{Cu}^{2+}$ lixiviado [\%] & TBZ lixiviado [\%] \\
\hline TBZ-Mt & n.c. & $13,6 \pm 0,7$ & n.c. & $8,3 \pm 0,2$ \\
TBZ-OMt55 & n.c. & $11,2 \pm 0,1$ & n.c. \\
Mt-Cu & $0,31 \pm 0,05$ & n.c. & $24 \pm 2$ & n.c. \\
TBZ-Mt-Cu & $0,28 \pm 0,04$ & $24,5 \pm 0,5$ & $0,9 \pm 0,1$ & $0,003^{*}$ \\
OMt55-Cu & $0,12 \pm 0,03$ & n.c. & $9,7 \pm 0,5$ & n.c. \\
TBZ-OMt55-Cu & $0,11 \pm 0,02$ & $24,5 \pm 0,5$ & $2,4 \pm 0,01$ & $0,005^{*}$ \\
EC-Mt-Cu & $0,24 \pm 0,03$ & $5,7 \pm 0,3$ & $0,7 \pm 0,1$ & $0,008^{*}$ \\
EC-OMt55-Cu & $0,09 \pm 0,01$ & $5,47 \pm 0,03$ & $3,1 \pm 0,4$ & $0,007^{*}$ \\
\hline
\end{tabular}

n.c.: no corresponde; $\left(^{*}\right)$ por debajo del límite de detección

Un comportamiento similar se encontró en la desorción de cisteína adsorbida en montmorillonitas saturadas con $\mathrm{Cu}^{2+}$ [Brigatti et al., 1999]. Allí los autores mostraron que la adsorción y desorción del aminoácido dependía del cation inorgánico interlaminar. Mientras que montmorillonitas saturadas con $\mathrm{Na}^{+}$y $\mathrm{Ca}^{2+}$ adsorbían y retenían pequeñas cantidades de cisteína y no daban lugar a la formación de complejos metal-cisteína, el aminoácido era adsorbido en cantidades mucho mayores y fuertemente retenido en montmorillonitas saturadas con $\mathrm{Cu}^{2+}$ [Brigatti et al., 1999]. En ese estudio se concluyó que la formación de complejos de coordinación estables entre el aminoácido y el metal de transición evitaba o retardaba la migración tanto del compuesto orgánico como del catión metálico. Este hecho no se observó en las muestras que contenían cationes interlaminares alcalino o alcalinotérreos.

El desarrollo de complejos estables $\mathrm{TBZ}^{-\mathrm{Cu}^{2+}}$ en la intercapa de los adsorbentes explicaría la disminución del porcentaje lixiviado del metal. Más aún, es probable que la distinta geometría que adopta el complejo en la intercapa de lugar a diferentes energías de interacción entre el metal y la matriz, lo que promovería diferentes porcentajes de lixiviado entre las muestras ( 0,9 y 3\% en Mt-Cu y OMt-Cu-TBZ, respectivamente).

Finalmente, la lixiviación de TBZ fue menor al límite de detección en todas las muestras $(0,0045 \mathrm{mg} / \mathrm{L})$, excepto para TBZ-Mt, sugiriendo que la modificación de la 
montmorillonita con surfactante y/o $\mathrm{Cu}^{2+}$ es recomendable para evitar la migración del fungicida desde el sólido a un medio acuoso.

\subsubsection{Caracterización de los materiales}

La Figura 6.5 muestra que luego de la adsorción de $\mathrm{Cu}^{2+}$ en OMt55 no hay cambios en el pico $\mathrm{d}_{001}$. Al igual que el material de partida, la deconvolución de este pico ancho, sugirió la existencia de dos espaciados basales, uno situado a 1,66 nm y el otro a 1,48 nm (Figura 4.8). Esto podría deberse a que el intercambio catiónico de $2 \mathrm{Na}^{+}$por $\mathrm{Cu}^{2+}$ ocurrió sin generar un ensanchamiento de la intercapa (nótese que el complejo $\mathrm{Cu}\left[\mathrm{H}_{2} \mathrm{O}\right]^{2+}$ en la muestra Mt-Cu da lugar a un espaciado basal de 1,26 nm, Sección 5.3.3). La presencia del metal en la intercapa, así como su interacción con TBZ, se confirmaron por la aparición de un nuevo componente en el pico $\mathrm{d}_{001}$ de la muestra TBZ-OMt55-Cu a 1,70 nm (Figura 6.5). $\mathrm{El}$ hecho de que el complejamiento del $\mathrm{Cu}^{2+}$ con el TBZ produzca un espaciado diferente al obtenido en la muestra TBZ-Mt-Cu (1,47 nm, Sección 5.3.3) se asignó a la diferencia de entorno químico de la intercapa (hidrofílico en Mt-Cu e hidrofóbico en OMt55-Cu) lo cual promovería diferentes geometrías en la esfera de coordinación de los complejos, induciendo los distintos espaciados basales obtenidos.

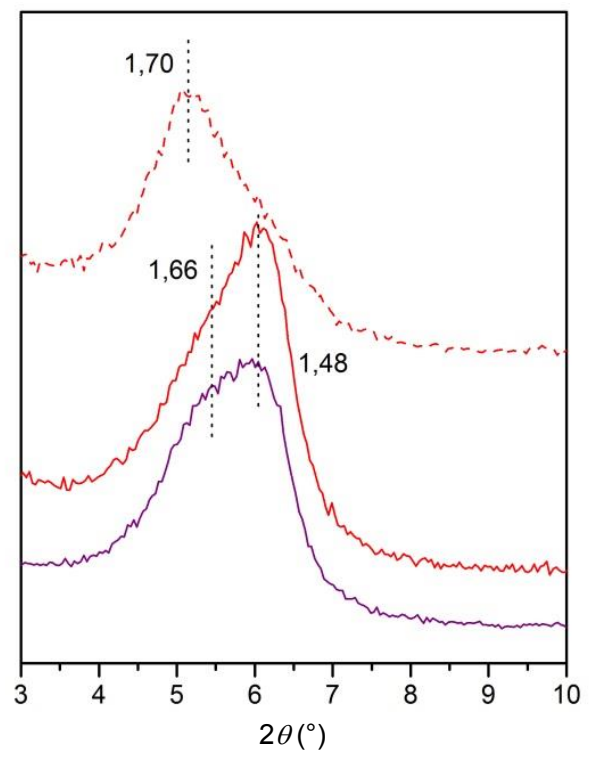

Figura 6.5 Difractogramas con el espaciado basal indicado (en nm) de (-) OMt55, (-) OMt55-Cu y (---) TBZ-OMt55-Cu.

La Figura 6.6 muestra las curvas de los ensayos térmicos, mientras que la Tabla 6.4 enumera las reacciones, temperaturas de los picos DTg y ATD, intervalos de temperaturas de los diferentes procesos así como también el porcentaje de masa perdida en cada uno. 

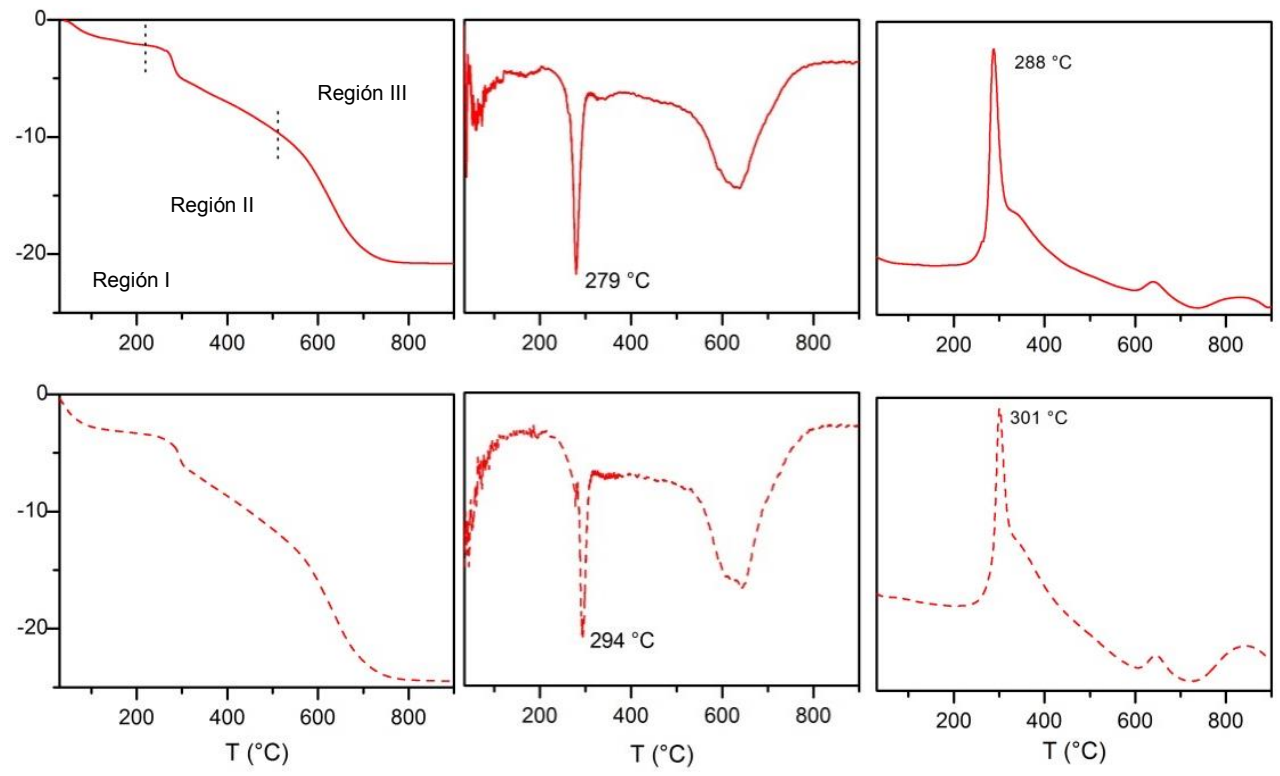

Figura 6.6 Curvas de análisis térmicos. De izquierda a derecha: Tg, DTg y ATD de (-) OMt55-Cu y (---) TBZ-OMt55-Cu.

En los Capítulos 3 y 4 se describió el comportamiento térmico de las muestras OMt55 y TBZ-OMt55. La muestra OMt55 presentó en la Región II, picos DTg y ATD (exotérmico) a 288 y $298{ }^{\circ} \mathrm{C}$, respectivamente (primer paso de oxidación); y a 419 y $411{ }^{\circ} \mathrm{C}$, respectivamente (segundo paso de oxidación) (Tabla 3.2). Algo similar ocurrió en el caso de la muestra TBZ-OMt55, cuya descomposición originó picos DTg y ATD (exotérmicos) a 304 y $307{ }^{\circ} \mathrm{C}$, respectivamente (primer paso de oxidación) y un pico DTg a $440{ }^{\circ} \mathrm{C}$ (segundo paso de oxidación) (Tabla 4.5). La destrucción del fungicida, ocurrió en forma solapada con la combustión de la amina que, al igual que en las otras organomontmorillonitas, ocurrió en dos etapas. Las muestras con $\mathrm{Cu}^{2+}$ en cambio, presentaron un único pico fino y bien pronunciado en la Región II, específicamente en la zona correspondiente a la primera etapa de oxidación, tanto en el DTg como en el ATD (Figura 6.6). Este pico, además se encontró a menor temperatura que el de las respectivas muestras sin $\mathrm{Cu}^{2+}$ : la OMt55-Cu, presentó sus picos DTg y ATD a 279 y $288{ }^{\circ} \mathrm{C}\left(9\right.$ y $10{ }^{\circ} \mathrm{C}$ más bajos que los de la OMt55, respectivamente, Tablas 3.2 y 6.4) y la TBZ-OMt55-Cu a 294 y $301{ }^{\circ} \mathrm{C}\left(10\right.$ y $6{ }^{\circ} \mathrm{C}$ más bajos que los de OMt55-TBZ, respectivamente, Tablas 4.5 y 6.4). Un comportamiento similar se encontró en el Capítulo 5, donde el producto de adsorción de IMZ en Mt-Cu presentó picos DTg y ATD correspondientes a la quema del imazalil, a temperaturas más bajas que los correspondientes picos en IMZ-Mt (Tablas 3.4 y 5.8). 
Tabla 6.4 Reacciones térmicas, picos DTg y ATD $\left[{ }^{\circ} \mathrm{C}\right]$ y porcentajes de pérdida de masa [\%] de OMt55-Cu y producto de adsorción de TBZ.

\begin{tabular}{|c|c|c|c|c|}
\hline & & & $\mathrm{OMt} 55-\mathrm{Cu}$ & TBZ-OMt55-Cu \\
\hline \multirow[t]{6}{*}{ Región I } & \multicolumn{2}{|l|}{ Intervalo de $\mathrm{T}$} & $30-210$ & $30-204$ \\
\hline & \multirow[t]{2}{*}{ Desorción de agua fisisorbida } & DTg & 63 & n.d. \\
\hline & & ATD (endo) & n.d. & n.d. \\
\hline & \multirow{2}{*}{$\begin{array}{l}\text { Deshidratación de cationes } \\
\text { interlaminares }\end{array}$} & DTg & n.d. & n.d. \\
\hline & & ATD (endo) & n.d. & n.d. \\
\hline & Pérdida de masa total & $\mathrm{Tg}$ & 2,0 & 3,3 \\
\hline \multirow[t]{7}{*}{ Región II } & \multicolumn{2}{|l|}{ Intervalo de $\mathrm{T}$} & $210-380$ & $204-405$ \\
\hline & \multirow{2}{*}{$\begin{array}{l}\text { 1er Paso de oxidación de contenido } \\
\text { orgánico. }\end{array}$} & DTg & $279 ; 334(\mathrm{~h})$ & 294 \\
\hline & & ATD (exo) & $288 ; 334(h)$ & $301 ; 335$ (h) \\
\hline & \multicolumn{2}{|l|}{ Intervalo de $\mathrm{T}$} & $380-501$ & $405-500$ \\
\hline & \multirow{2}{*}{$\begin{array}{l}2 \text { do Paso de oxidación de contenido } \\
\text { orgánico. }\end{array}$} & DTg & n.d. & n.d. \\
\hline & & ATD (exo) & n.d. & n.d. \\
\hline & Pérdida de masa total & $\operatorname{Tg}$ & 7,2 & 8,2 \\
\hline \multirow[t]{6}{*}{ Región III } & \multicolumn{2}{|l|}{ Intervalo de $\mathrm{T}$} & $501-800$ & $500-843$ \\
\hline & \multirow[t]{2}{*}{ Deshidroxilación } & DTg & 631 & $604(h) ; 642$ \\
\hline & & ATD (endo) & 641 & 646 \\
\hline & \multirow[t]{2}{*}{ Oxidación de restos carbónicos } & DTg & n.d. & n.d. \\
\hline & & ATD (exo) & n.d. & n.d. \\
\hline & Pérdida de masa total & $\mathrm{Tg}$ & 11,3 & 12,4 \\
\hline
\end{tabular}

n.d.: no determinado; (h) hombro

Aunque la pérdida de masa continúe a mayores temperaturas (Figura 6.6) la combustión del contenido orgánico (ODTMA y TBZ, ya que no es posible distinguirlos), en presencia del metal, ocurre principalmente en el primer intervalo de temperaturas de la Región II. Este fenómeno se puede explicar por la capacidad del ion $\mathrm{Cu}^{2+}$ de actuar como catalizador en la reacción de oxidación de compuestos orgánicos [Yermiyahu et al., 2002; Wen-Chen Tsai y Jiang-Jen Lin, 2010; Nzihou y Stanmore, 2013; Azargohar et al., 2014]. Yermiyahu et al. [2002] sugirieron que el tratamiento térmico del producto de adsorción del colorante aniónico congo-red en una montmorillonita saturada con $\mathrm{Cu}^{2+}$, conduce a la formación de radicales cationes que catalizan la oxidación del colorante, la volatilización de los productos 
y colapso de la intercapa a temperaturas menores a los $500{ }^{\circ} \mathrm{C}$, diferenciándose del comportamiento térmico de los productos de adsorción del colorante en montmorillonitas saturadas con otros cationes inorgánicos $\left(\mathrm{Na}^{+}, \mathrm{Cs}^{+}, \mathrm{Mg}^{2+}, \mathrm{Al}^{3+}\right.$ y $\left.\mathrm{Fe}^{3+}\right)$ donde se observó la formación de diferentes estructuras de carbón en la intercapa. Esta disminución en el contenido de carbón debido a la oxidación y volatilización del ODTMA y TBZ catalizada por el ion $\mathrm{Cu}^{2+}$, explicaría también el corrimiento hacia mayores temperaturas de los picos de deshidroxilación (DTg y ATD) de las muestras con $\mathrm{Cu}^{2+}$ respecto a las mismas sin el metal (por ejemplo, el pico DTg se corre de 600 a $631{ }^{\circ} \mathrm{C}$ en OMt55 y OMt55-Cu, respectivamente; y de 603 a $642{ }^{\circ} \mathrm{C}$ en TBZ-OMt55 y TBZ-OMt55-Cu, respectivamente, Tablas 3.2, 4.5 y 6.4).

A partir de los análisis térmicos y termogravimétricos, no es posible extraer información acerca de los sitios de adsorción de TBZ. Para ello, se recurrió a una técnica específica que permite caracterizar el entorno químico del $\mathrm{Cu}^{2+}$ en las muestras, como es la EPR. Los espectros EPR en banda X se determinaron con el objeto de obtener información acerca de la coordinación de los iones $\mathrm{Cu}^{2+}$ en la estructura de la montmorillonita y de la organomontmorillonita en primera instancia, y de establecer si la complejación del TBZ con el metal efectivamente tiene lugar, en segunda instancia.

Los resultados EPR se muestran en la Figura 6.7. En primera medida, el hecho de que las muestras presenten señal, indica que el metal se encuentra con estado de oxidación 2+ ya que el ion $\mathrm{Cu}^{+}$no genera señal EPR por ser diamagnético. Esto permite descartar reacciones oxido-reducción tanto durante el intercambio del mismo al preparar los adsorbentes, como durante la adsorción del fungicida.

$\mathrm{El} \mathrm{Cu}^{2+}$ presente en $\mathrm{Mt}-\mathrm{Cu}$ y $\mathrm{OMt} 55-\mathrm{Cu}$ y en sus correspondientes productos de adsorción, presentan dos estructuras finas alrededor de valores de $g$ de 2,04 y 2,12 junto a otra región hiperfina no resuelta para valores de $g$ entre 2,2-2,7 de diferente intensidad según la muestra.

La señal a $g=2,12$ se asoció a iones $\mathrm{Cu}^{2+}$ hidratados o coordinados con grupos hidroxilo en la intercapa de la Mt-Cu [Heller-Kallai y Mosser 1995; He et al., 2001]. En el caso de la muestra OMt55-Cu, la contribución de $g=2,13$ no pudo ser bien resuelta debido al ensanchamiento de la señal de EPR asociado a la presencia de diferentes sitios a los cuales los iones paramagnéticos pueden unirse, teniendo en cuenta que la intercalación del surfactante generó un entorno químico heterogéneo (que inclusive se tradujo en dos espaciados interlaminares diferentes). La presencia de los cationes ODTMA en los sitios de carga permanente afectó el número de sitios disponibles e impidió, tanto desde el punto de 
vista cinético como termodinámico, el subsiguiente intercambio de $\mathrm{Na}^{+}$por $\mathrm{Cu}^{2+}$. Esto se tradujo en un menor contenido de $\mathrm{Cu}^{2+}$ de la muestra OMt55-Cu $(0,124 \mathrm{mmol} / \mathrm{g})$ en comparación con el de $\mathrm{Mt}-\mathrm{Cu}(0,309 \mathrm{mmol} / \mathrm{g})$. Más aún, es esperable que algunos cationes $\mathrm{Cu}^{2+}$ estén unidos a los sitios de carga variable (bordes) [Ma et al., 2016], aunque este comportamiento no pudo confirmarse.

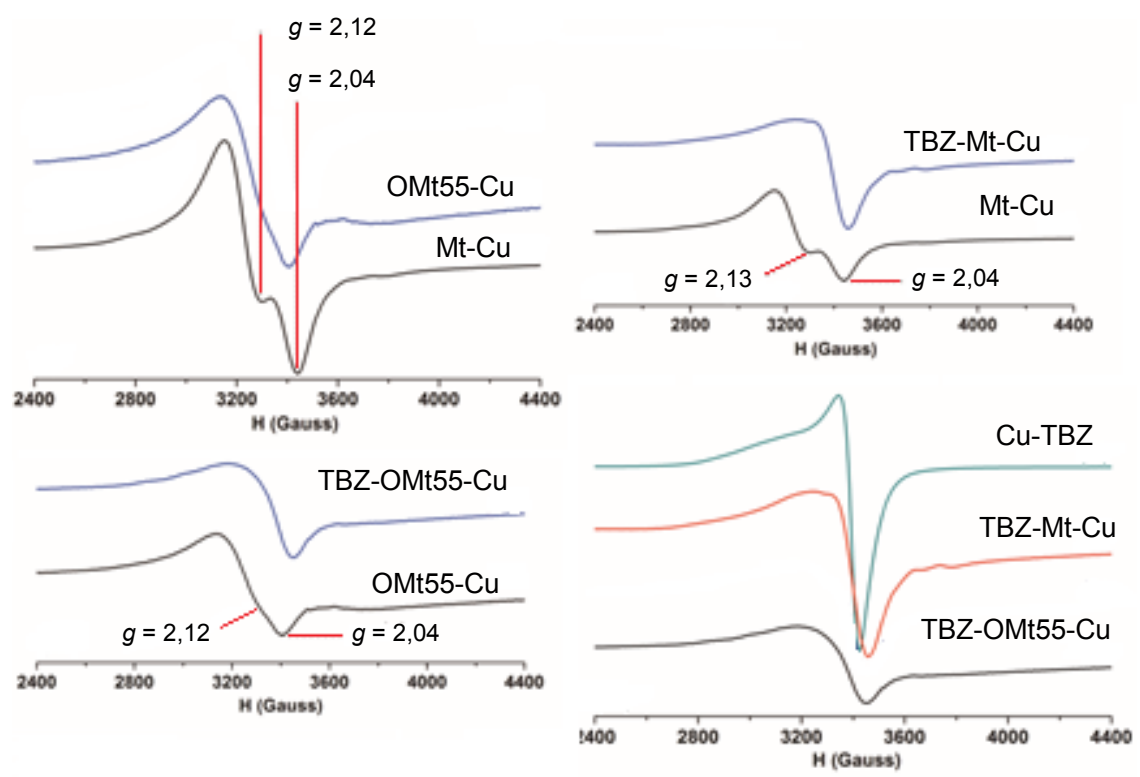

Figura 6.7 Espectros EPR de las muestras indicadas.

La otra señal, a $g=2,04$, se atribuyó a la adsorción específica de $\mathrm{Cu}^{2+}$ en las cavidades hexagonales de las capas siloxánicas, de acuerdo a resultados reportados en [He et al., 2001].

Los cambios que originó la adsorción de TBZ en los espectros EPR fueron los mismos independientemente del adsorbente. El incremento de la componente hiperfina en el espectro, indica que efectivamente la molécula se coordina con el ion metálico. Esto significa que durante la adsorción tuvo lugar una reacción de intercambio de ligando, donde algunas moléculas de agua o grupos hidroxilo presentes en la esfera de coordinación del ion fueron reemplazados por el ligando TBZ [J.M. Lázaro-Martínez et al., 2015] -de acuerdo con la contracción del espaciado basal que se observó para Mt-Cu-TBZ en relación a TBZMt-. Además, puede afirmarse que la quelación del metal se establece a través de los átomos de nitrógeno de los grupos imidazol y tiazol, ya que los espectros EPR de los productos de adsorción presentaron un corrimiento a mayores campos de las líneas. Esto se explica por el hecho de que el nitrógeno tiende a incrementar los valores de $A_{\text {II }}$ y reducir los de $g_{I I}$ en comparación a la esfera de coordinación del $\mathrm{Cu}^{2+}$ en los adsorbentes, donde los 
iones paramagnéticos se encuentran coordinados a los átomos de oxígeno [Donoso et al., 2013; Lázaro-Martínez et al., 2013]. Sin embargo, la gran densidad de iones $\mathrm{Cu}^{2+}$ afectan la resolución de la estructura hiperfina haciendo imposible la obtención de mayor información relacionada a los ligandos de los centros metálicos. La proximidad entre centros paramagnéticos $\mathrm{Cu}^{2+}$ (distancias menores a 0,1-0,15 nm) [Rizzi et al., 2016] ensancha la señal EPR por las interacciones spin-orbital y de intercambio. Un ejemplo de este fenómeno se observó en el espectro EPR del complejo sintetizado Cu-TBZ (Figura 6.7).

\subsection{Conclusiones parciales}

En este capítulo se obtuvo una montmorillonita intercambiada con ODTMA al 55 \%CIC y luego con $\mathrm{Cu}^{2+}$ al 30 \%CIC. Su caracterización indicó que la incorporación del metal ocurre en los sitios de carga permanente por intercambio entre los iones $\mathrm{Na}^{+}$ remanentes y el $\mathrm{Cu}^{2+}$.

Se evaluó la capacidad de remover TBZ en solución acuosa tanto en estado puro como en un efluente real. En el primer caso, se realizaron isotermas de adsorción reduciendo la relación sólido/solución hasta $0,1 \mathrm{~g} / \mathrm{L}$. Esto llevó al aumento de la cantidad adsorbida por unidad de masa (en las muestras con $\mathrm{Cu}^{2+}$ hasta cientos de $\mathrm{mg} / \mathrm{g}$ ) pero fue acompañado de una disminución de la eficiencia de la remoción. Se observó que hay una dependencia de la cantidad adsorbida con la cantidad de centros $\mathrm{Cu}^{2+}$ en la suspensión. Sin embargo, no sólo la cantidad sino también la disponibilidad de los metales para interactuar con el TBZ definieron el desempeño de los materiales como adsorbentes. En ese sentido, el hecho de que la muestra $\mathrm{Mt}-\mathrm{Cu}$ presentara mayor contenido de $\mathrm{Cu}^{2+}$ por unidad de masa, y capacidad de dispersarse en agua, condujo a un mejor desempeño respecto a OMt55-Cu y eficiencias de remoción elevadas, aun a dosis bajas de sólido (86\% para la relación sólido/solución $=0,1 \mathrm{~g} / \mathrm{L}$ ). Al realizar estudios de adsorción-desorción de TBZ presente en un efluente real, se obtuvieron resultados semejantes, donde la muestra Mt-Cu fue la que presentó mayor capacidad de remoción del fungicida. Se evaluaron distintas condiciones para promover la desorción del fungicida, y se observó que en medio básico se favorecía, para las muestras Mt y OMt55, debido a la deprotonación del $\mathrm{TBZH}^{+}$unido a la superficie y a la competencia de $\mathrm{Na}^{+}$por $\mathrm{TBZH}^{+}$en los sitios de carga permanente; mientras que en las muestras con $\mathrm{Cu}^{2+}$ la desorción fue muy baja en todas las condiciones ( $0,31 \%$ en OMt55-Cu y $0,004 \%$ en Mt-Cu). 
La caracterización de los materiales y productos de adsorción mediante DRX, EPR y análisis térmicos, permitieron confirmar que los sitios activos de adsorción del TBZ en Mt$\mathrm{Cu}$ y $\mathrm{OMt} 55-\mathrm{Cu}$ corresponden a los centros metálicos, y homologa el mecanismo propuesto en el Capítulo 5.

Además los datos de lixiviación tanto del metal como de TBZ en las muestras con $\mathrm{Cu}^{2+}$ permiten proponer a estas muestras para el tratamiento de efluentes de la producción frutícola. 
En este trabajo de tesis se obtuvieron y caracterizaron, en una primera parte organomontmorillonitas (OMts) mediante intercambio catiónico con octadeciltrimetil amonio (ODTMA) -una amina cuaternaria con una cadena alquílica de 18 carbonos- tanto en una montmorillonita nacional $(\mathrm{Mt})$ como en una de referencia internacional. Los materiales obtenidos, con diferentes contenidos de ODTMA, se caracterizaron mediante DRX, potencial zeta, análisis térmico, FTIR, XPS y simulaciones computacionales. Estas técnicas permitieron dar cuenta de que, a porcentajes menores al $100 \% \mathrm{CIC}$, el ODTMA se une preferentemente a sitios de carga permanente (intercapa), mientras que a porcentajes por encima del $100 \%$ CIC, las cadenas alquílicas recubren la superficie externa de la montmorillonita. En todos los casos, el tamaño de las partículas -medido como diámetro aparente- crece en un orden de magnitud respecto a la montmorillonita natural, lo cual permite su separación posterior de la solución acuosa.

El mecanismo de adsorción de los fungicidas tiabendazol (TBZ) e imazalil (IMZ) ambos de carácter básico- es el intercambio catiónico de la forma protonada de los fungicidas por $\mathrm{Na}^{+}$, siendo los sitios de carga permanente y de superficie externa los sitios activos de unión de éstos. En el caso de IMZ, cuando la isoterma se realizó ajustando el pH a 7, la cantidad de $\mathrm{IMZH}^{+}$superó el 100 \%CIC. En estas condiciones se comprobó que los sitios de borde también participaron en la adsorción del fungicida. 
El estudio de adsorción de TBZ e IMZ en organo-montmorillonitas reveló que la incorporación de cadenas alquílicas no es beneficiosa en términos de generación de nuevos sitios activos para la unión de los compuestos, ya que la amina no estuvo involucrada directamente en la unión de éstos.

En el caso del IMZ, a concentraciones bajas de fungicida, la presencia de ODTMA significó la disminución de $\mathrm{Na}^{+}$y sus esferas de hidratación y consecuentemente, una disminución de $\mathrm{IMZH}^{+}$en la superficie del sólido con respecto a Mt. En estos casos, la muestra Mt presentó un mejor desempeño respecto a las OMts. Sin embargo, a concentraciones altas de IMZ, el mecanismo de intercambio catiónico no puede explicar la cantidad adsorbida de IMZ, y es otro mecanismo el que tiene lugar. Al evidenciarse que las cadenas alquílicas no interaccionan con el IMZ, se propuso que el sitio de adsorción es la superficie siloxánica (tanto de la intercapa como de la superficie externa), que en ausencia de cationes inorgánicos y en presencia de cadenas alquílicas es de naturaleza hidrofóbica. Así, las cadenas alquílicas actúan como pilares formando una galería a la cual el IMZ en su forma neutra puede ingresar y unirse mediante interacciones de van der Waals con la superficie siloxánica. Este efecto fue positivo a concentraciones altas de IMZ y cantidades bajas de ODTMA, donde la cantidad adsorbida de IMZ en las OMts (particularmente aquellas intercambiadas entre 39 y 75 \% CIC) fue mayor que en Mt. Cuando la cantidad de ODTMA es alta (porcentajes mayores al 100\%CIC) la superficie siloxánica está totalmente cubierta y consecuentemente, los sitios disponibles para la adsorción del IMZ disminuyen. El mecanismo de adsorción de IMZ neutro en la superficie siloxánica estuvo favorecido a $\mathrm{pH}$ básicos, donde esta es la especie mayoritaria del fungicida en solución.

La dependencia de la cantidad de IMZ adsorbida con el pH y con la concentración de IMZ, sugiere que el uso de montmorillonitas u organo-montmorillonitas en el tratamiento de efluentes es recomendable en función de estos parámetros.

En el caso del TBZ, la incorporación de ODTMA no mejoró la capacidad de adsorción de las organo-montmorillonitas respecto a la montmorillonita natural. La presencia de los cationes ODTMA en los sitios de carga permanente disminuyó el número de sitios disponibles para la unión del fungicida, y por ello la capacidad de remoción de TBZ es menor cuanto mayor es la cantidad de ODTMA en el sólido. La presencia de ODTMA en porcentajes mayores al $100 \% \mathrm{CIC}$, al igual que en el IMZ, generó un mecanismo diferente. En este caso, se comprobó que se establece un intercambio catiónico entre $\mathrm{TBZH}^{+}$y $\mathrm{ODTMA}^{+}$en la superficie externa. Este mecanismo estuvo favorecido por las fuertes energías de interacción entre la superficie siloxánica negativa y la especie protonada del 
fungicida, y por las fuerzas repulsivas entre los cationes ODTMA $^{+}$en la superficie externa. En este último caso el producto de adsorción es inestable, presentando porcentajes de desorción elevados en agua.

Estos resultados permitieron rectificar la hipótesis del trabajo de investigación que sugería que la incorporación de cadenas alquílicas mejoraría la capacidad de adsorción de compuestos orgánicos, en las organo-montmorillonitas respecto a las montmorillonitas naturales, y buscar nuevas estrategias para aumentar la capacidad de adsorción del material.

Las simulaciones computacionales de TBZ en la superficie externa de Mt planteó la posible -aunque teórica- existencia de un complejo formado por la coordinación de TBZ con el cation $\mathrm{Na}^{+}$. Este resultado, sumado a los reportes bibliográficos de complejos estables donde el TBZ actúa como ligando bidentado, sugirieron que la incorporación de un metal de transición en la estructura de la Mt, mejoraría la adsorción del fungicida. Es por ello que, en la segunda parte de esta tesis, se obtuvieron materiales por intercambio catiónico, de la montmorillonita natural y de una organomontmorillonita con $\mathrm{Cu}^{2+}(\mathrm{Mt}-\mathrm{Cu}$ y OMt55-Cu, respectivamente) y se evaluó la adsorción de TBZ e IMZ.

Los experimentos cinéticos, revelaron que en los primeros 10 min de contacto, más del $98 \%$ de TBZ $\left(C_{i}=15 \mathrm{mg} / \mathrm{L}\right)$ o IMZ $\left(C_{i}=40 \mathrm{mg} / \mathrm{L}\right)$ fue removido cuando se utilizó una relación sólido/solución de $1 \mathrm{~g} / \mathrm{L}$ de Mt-Cu. Entre los modelos matemáticos evaluados, aquellos que suponen la quimisorción del adsorbato como paso determinante de la velocidad fueron los que mejor se ajustaron a los datos experimentales. Desde el punto de vista cinético el TBZ estuvo favorecido respecto al IMZ, aunque en equilibrio ocurrió lo contrario.

Las isotermas de adsorción revelaron que los centros metálicos en estas muestras, actuaron como sitios activos en la unión de los fungicidas, donde el mecanismo implicó el desplazamiento de moléculas de agua o grupos hidroxilos de la esfera de coordinación del metal, por parte del TBZ o IMZ. En el mecanismo propuesto, los fungicidas se coordinan a través del átomo de nitrógeno de los anillos bencimidazol y tiazol para el TBZ e imidazol para el segundo. Los fungicidas fueron removidos en más de un $90 \%$ en todo el intervalo de concentraciones analizado, cuando se utilizó una relación sólido/solución $=1 \mathrm{~g} / \mathrm{L}$, lo que promovió el uso de una menor relación sólido/solución. La disminución de esta relación, aunque mejoró la cantidad adsorbida por unidad de masa llevó a una disminución en la eficiencia de remoción. El material Mt-Cu mostró ser eficiente inclusive a dosis de adsorbente de 0,1 g/L y es posible su utilización en efluentes conteniendo ambos fungicidas, ya sea en forma secuencial o simultánea. 
A partir de ensayos de EPR se pudo comprobar que el mecanismo propuesto fue correcto, tanto para la adsorción de TBZ en la montmorillonita como en la organomontmorillonita intercambiadas con $\mathrm{Cu}^{2+}$. La formación de complejos estables en la intercapa de las muestras llevó a porcentajes de lixiviación muy bajos tanto del metal como del fungicida.

Además, estas muestras presentaron porcentajes de remoción de TBZ importantes cuando este proviene de un efluente real. Aunque es necesario seguir investigando y realizando ensayos, estas muestras presentan un gran potencial para su aplicación tecnológica en el tratamiento de efluentes acuosos provenientes de la producción frutícola. 


\section{Referencias}

Abou-El-Sherbini, K. S., \& Hassanien, M. M. (2010). Study of organically-modified montmorillonite clay for the removal of copper (II). Journal of hazardous materials, 184(1), 654-661.

Aharonson N. \& Kafkafi U. (1975a). Adsorption, mobility, and persistente of thiabendazole and metil 2benzimidazolecarbamate in soils, Journal of Agricultural and Food Chemistry 23720.

Aharonson, N., \& Kafkafi, U. (1975b). Adsorption of benzimidazole fungicides on montmorillonite and kaolinite clay surfaces. Journal of agricultural and food chemistry, 23(3), 434-437.

Akpomie, K. G., \& Dawodu, F. A. (2014). Efficient abstraction of nickel (II) and manganese (II) ions from solution onto an alkaline-modified montmorillonite. Journal of Taibah University for Science, 8(4), 343356.

Allen, S. J., Whitten, L. J., Murray, M., Duggan, O., \& Brown, P. (1997). The adsorption of pollutants by peat, lignite and activated chars. Journal of Chemical Technology and Biotechnology, 68(4), 442-452.

Al-Qunaibit, M. H., Mekhemer, W. K., \& Zaghloul, A. A. (2005). The adsorption of Cu (II) ions on bentonite—a kinetic study. Journal of colloid and interface science, 283(2), 316-321.

Altamirano J.E., Franco R. y Bovi Mitre M.G. (2004). Modelo epidemiológico para el diagnóstico de intoxicación aguda por plaguicidas. Rev. Toxicol. 21, 98-102.

Anderson, S.J., \& Sposito, G. (1991). Cesium-adsorption method for measuring accessible structural surface charge. Soil Science Society of America Journal 55, 1569-1576.

Annabi-Bergaya, F., Cruz, I.M., Gatineau, L., Fripiat, J.J., (1981). Adsorption of alcohols by smectites. Clay Minerals 16, 115-122.

Azargohar, R., Nanda, S., Kozinski, J. A., Dalai, A. K., \& Sutarto, R. (2014). Effects of temperature on the physicochemical characteristics of fast pyrolysis bio-chars derived from Canadian waste biomass. Fuel, 125, 90-100.

Baldassari, S., Komarneni, S., Mariani, E., \& Villa, C. (2006). Microwave versus conventional preparation of organoclays from natural and synthetic clays. Applied Clay Science, 31(1), 134-141. 
Balomenou, G., Stathi, P., Enotiadis, A., Gournis, D., \& Deligiannakis, Y. (2008). Physicochemical study of amino-functionalized organosilicon cubes intercalated in montmorillonite clay: H-binding and metal uptake. Journal of colloid and interface science, 325(1), 74-83.

Barreto, A. S., Aquino, A., Silva, S. C., de Mesquita, M. E., Calhorda, M. J., Saraiva, M. S., \& Navickiene, S. (2011). A novel application of mesoporous silica material for extraction of pesticides. Materials Letters, 65(9), 1357-1359.

Bergaya, F., Lagaly, G., (2001). Surface modifications of clay minerals. Applied Clay Science, 19, 1-3.

Bianchi, A. E., Fernández, M., Pantanetti, M., Viña, R., Torriani, I., Sánchez, R. T., \& Punte, G. (2013). ODTMA+ and HDTMA+ organo-montmorillonites characterization: New insight by WAXS, SAXS and surface charge. Applied Clay Science, 83, 280-285.

Bishop, J. L., Pieters, C. M., \& Edwards, J. O. (1994). Infrared spectroscopic analyses on the nature of water in montmorillonite. Clays and Clay Minerals, 42(6), 702-716.

Boeva, N. M., Bocharnikova, Y. I., Nasedkin, V. V., Belousov, P. E., \& Demidenok, K. V. (2013). Thermal analysis as an express method for assessing the quality and quantity of natural and synthesized organoclays. Nanotechnologies in Russia, 8(3-4), 205-208.

Bovi Mitre, M. G., Wierna, N. R., Retamozo, Z., \& Ruggeri, M. A. (2004). Curva de Degradación del Plaguicida Imazalil en Frutos Maduros de Pomelos durante el Almacenamiento en Frio. Información tecnológica, 15(3), 67-70.

Breakwell, I.K., Homer, J., Lawrence, M.A.M., McWhinnie, W.R., (1995). Studies of organophilic clays: the distribution of quaternary ammonium compounds on clay surfaces and the role of impurities. Polyhedron $14,2511-2518$.

Breen, C., \& Loughlin, H. (1994). The competitive adsorption of methylene blue on to $\mathrm{Na}$ montmorillonite from binary solution with n-alkylytrimethyldammonium surfactants. Clay Minerals, 29(5), 775-783.

Breen, C., \& Rock, B. (1994). The Competitive Adsorption of Methylene Blue on to Montmorillonite from Binary Solution wih Thioflavin T, Proflavine and Acridine Yellow. Steady-State and Dynamic Studies. Clay Minerals, 29(17), 189.;

Brigatti, M. F., Lugli, C., Montorsi, S., \& Poppi, L. (1999). Effects of exchange cations and layer-charge location on cysteine retention by smectites. Clays and Clay Minerals, 47(5), 664-671. 
Briggs, D., \& Beamson, G. (1992). Primary and secondary oxygen-induced C1s binding energy shifts in Xray photoelectron spectroscopy of polymers. Analytical chemistry, 64(15), 1729-1736.

Brindley, G. W., \& Moll, W. F. (1965). Complexes of natural and synthetic ca-montmorillonites with fatty acids (CLAY-ORGANIC STUDIES-9). American Mineralogist, 50(9), 1355.

Brown, L., Seaton, K., Mohseni, R., \& Vasiliev, A. (2013). Immobilization of heavy metals on pillared montmorillonite with a grafted chelate ligand. Journal of hazardous materials, 261, 181-187.

Brtáňová, A., Madejová, J., Bizovská, V., \& Komadel, P. (2014). Utilization of near infrared spectroscopy for studying solvation properties of Cu-montmorillonites. Spectrochimica Acta Part A: Molecular and Biomolecular Spectroscopy, 123, 385-391.

CAFI, Cámara Argentina de Fruticultores, Producción de peras y manzanas en Argentina 19 de mayo de 2014, disponible en: http:/ /www.cafi.org.ar/produccion-de-peras-y-manzanas-en-argentina/-

Carra, I., Malato, S., Jiménez, M., Maldonado, M. I., \& Pérez, J. S. (2014). Microcontaminant removal by solar photo-Fenton at natural $\mathrm{pH}$ run with sequential and continuous iron additions. Chemical Engineering Journal, 235, 132-140.

Castillo, L. E., Ruepert, C., \& Solis, E. (2000). Pesticide residues in the aquatic environment of banana plantation areas in the north Atlantic zone of Costa Rica. Environmental Toxicology and Chemistry, 19(8), 1942-1950.

Celis, R., Hermosin, M.C., Cornejo, J., (2000). Heavy metal adsorption by functionalized clays. Environmental Science \& Technology 34, 4593-4599.

Cheung, C. W., Chan, C. K., Porter, J. F., \& McKay, G. (2001). Film-pore diffusion control for the batch sorption of cadmium ions from effluent onto bone char. Journal of colloid and interface science, 234(2), 328336.

Cheung, C. W., Porter, J. F., \& McKay, G. (2001). Sorption kinetic analysis for the removal of cadmium ions from effluents using bone char. Water Research, 35(3), 605-612.

Choy, K. K., Ko, D. C., Cheung, C. W., Porter, J. F., \& McKay, G. (2004). Film and intraparticle mass transfer during the adsorption of metal ions onto bone char.Journal of colloid and interface science, 271(2), 284-295.

Chun Hui Zhou, Li Zhi Zhao, Ai Qin Wang, Tian Hu Chen, Hong Ping He. (2016) Current fundamental and applied research into clay minerals in China. Applied Clay Science 119, 3-7; 
CIAFA, Cámara de la Industria Argentina de Fertilizantes y Agroquímicos, Manual Fitosanitario, disponible en: http://www.manualfitosanitario.com/producto_comercial.php

Codex Alimentarius, disponible en: http://www.fao.org/fao-whocodexalimentarius/standards/pestres/es/

Colodner, A. (2011). Optimización de las técnicas de aplicación de productos fitosanitarios en la línea de empaque de manzanas (Malus domestica, Borkh), Tesis para optar al grado academico de Master of Scientia de Fruticultura de clima templado-frío, Universita degli Studi di Bologna, INTA, Alto Valle, General Roca.

Coscolla, R., (2008). Residuos de productos fitosanitarios. Servicio de sanidad vegetal y proteccion fitosanitaria. Direccion general de investigacion y tecnologia agroalimentaria. Generalitat Valenciana.

Craviotti, C.; Palacios P.; Soleno R.. (2010) Territorios y mercados globales: Las firmas agroexportadoras citrícolas del noreste argentino; Mundo Agrario, vol. 10, n 20, primer semestre de 2010. ISSN 1515-5994

Czímerová, A., Bujdák, J., \& Dohrmann, R. (2006). Traditional and novel methods for estimating the layer charge of smectites. Applied Clay Science, 34(1), 2-13.

de Paiva, L. B., Morales, A. R., \& Díaz, F. R. V. (2008). Organoclays: properties, preparation and applications. Applied Clay Science, 42(1), 8-24.

COFECyT, 2008. Consejo Federal de Ciencia y Tecnología, Debilidades y desafios tecnologicos del sector productivo frutas cítricas (limón, mandarina y naranja), Corrientes, Entre Ríos y Tucumán, disponible en: http://www.cofecyt.mincyt.gov.ar/pcias_pdfs/corrientes/UIA_frutas_cit_08.pdf

Devereux, M., Shea, D. O., Kellett, A., McCann, M., Walsh, M., Egan, D., \& Müller-Bunz, H. (2007). Synthesis, X-ray crystal structures and biomimetic and anticancer activities of novel copper (II) benzoate complexes incorporating 2-(4'-thiazolyl) benzimidazole (thiabendazole), 2-(2-pyridyl) benzimidazole and 1, 10-phenanthroline as chelating nitrogen donor ligands. Journal of inorganic biochemistry, 101(6), 881-892.

Ding, Z., \& Frost, R. L. (2004). Study of copper adsorption on montmorillonites using thermal analysis methods. Journal of colloid and interface science, 269(2), 296-302.

Dirección de Minería de San Juan, Minería Extractiva, Bentonitas, disponible en: http://www.mineria.sanjuan.gov.ar/recursos/min_extract_pdf/Bentonitas.PDF

Domínguez, J.M., \& Schifter, I.; (2013) Las arcillas: el barro noble; Colección La ciencia para todos; Editor: Fondo de Cultura Económica; Instituto Latinoamericano de la Comunicación Educativa, España; http://bibliotecadigital.ilce.edu.mx/sites/ciencia/volumen3/ciencia3/109/html/arcillas.html 
Doner, H.E., Mortland, M.M., (1969). Benzene complexes with Cu(II)-montmorillonite. Science 66, $1406-1407$.

Donoso, J. P., Magon, C. J., Lima, J. F., Nascimento, O. R., Benavente, E., Moreno, M., \& Gonzalez, G. (2013). Electron Paramagnetic Resonance Study of Copper-Ethylenediamine Complex Ion Intercalated in Bentonite. The Journal of Physical Chemistry C, 117(45), 24042-24055.

Draoui, K., Denoyel, R., Chgoura, M., \& Rouquerol, J. (1998). Adsorption of Pesticides on Soil Components. Journal of thermal analysis and calorimetry, 51(3), 831-839.

Dultz, S., Riebe, B., \& Bunnenberg, C. (2005). Temperature effects on iodine adsorption on organo-clay minerals: II. Structural effects. Applied Clay Science, 28(1), 17-30.

Dunia, E., Elisenda Pulido, M., Cristina Fernández, R., Ortega, J. A., Sebastián, O., \& José, M. (2011). Degradation and detoxification of banana postharvest treatment water using advanced oxidation techniques. Green and Sustainable Chemistry.

Dweck, J. (2008). Qualitative and quantitative characterization of Brazilian natural and organophilic clays by thermal analysis. Journal of Thermal Analysis and Calorimetry, 92(1), 129-135.

El Cronista, Gracias a los transgénicos, la Argentina ya está entre los primeros 10 países agrícolas, 17 de enero de 2012, disponible en: http://www.cronista.com/negocios/Gracias-a-los-transgenicos-la-Argentinaya-esta-entre-los-primeros-10-paises-agricolas-20120117-0059.html.

Emmerich, K.; Plöze, M.; Kahr, G. (2001) Reversible collapse and Mg2+ release of de- and rehydroxylated homoionic cis-vacant montmorillonites. Applied Clay Science, 19, 143-154.

Ennajih, H., Gueddar, H., El Kadib, A., Bouhfid, R., Bousmina, M. \& Essassi, E. (2012) Intercalation of nickel and cobalt thiabendazole complexes into montmorillonite. Applied Clay Science 65-66, 139-142.

EPA, Environmental Protection Agency (1990), Method 1312: Synthetic precipitation leaching procedure, disponible en: https://www.epa.gov/sites/production/files/2015-12/documents/1312.pdf

Escudey, M., Galindo, G., \& Ervin, J. (1986). Effect of iron oxide dissolution treatment on the isoelectric point of allophanic soils. Clays and Clay Minerals, 34(1), 108-110.

EU Pesticides database, disponible en: http://ec.europa.eu/food/plant/pesticides/eu-pesticidesdatabase/public/?event=pesticide.residue.CurrentMRL\&language $=\mathrm{EN}$

EUROPEAN COMMISSION, Review report for de active substance thiabendazole. 7603/VI/97-final, 1-41 (2001). 
F. Annabi-Bergaya, M. I. Cruz, L. Gatineau and J. J. Fripiat, (1979) Adsorption of alcohols by smectites: i. Distinction between internal and external surfaces, Clay minerals, 14, 249.

Fabiani A., Mika R., Larocca L. Y Anderson C.; (2012) Manual Para Productores De Naranja Y Mandarina De La Region Del Rio Uruguay; Capítulo 13. Cosecha, Transporte Y Postcosecha; Diversificion Productiva Manual Serie A No 2; 2da Ed. Isbn: 950-9853-72-0; PRODIP - Proyecto de Diversificación Productiva INTA - Estación Experimental Agropecuaria Concordia

Fan, H., Zhou, L., Jiang, X., Huang, Q., \& Lang, W. (2014). Adsorption of Cu 2+ and methylene blue on dodecyl sulfobetaine surfactant-modified montmorillonite. Applied Clay Science, 95, 150-158.

Farmer, V.C., Mortland, M.M., (1966). An infrared study of the coordination of pyridine and water to exchangeable cations in montmorillonite and saponite. Journal of the Chemical Society A, 344-351.

Fatimah, I., \& Huda, T. (2013). Preparation of cetyltrimethylammonium intercalated Indonesian montmorillonite for adsorption of toluene. Applied Clay Science, 74, 115-120.

Favre, H., \& Lagaly, G. (1991). Organo-bentonites with quaternary alkylammonium ions. Clay Minerals, 26(1), 19-32.

Feldkamp, J.R., White, J.L., (1979). Acid-base equilibria in clay suspension. Journal of Colloid and Interface Science 69, 97-106.

Foo, K. Y., \& Hameed, B. H. (2010). Detoxification of pesticide waste via activated carbon adsorption process. Journal of hazardous materials, 175(1), 1-11.

Fruhstorfer, P., Schneider, R. J., Weil, L., \& Niessner, R. (1993). Factors influencing the adsorption of atrazine on montmorillonitic and kaolinitic clays. Science of the total environment, 138(1-3), 317-328.

Gamba, M., Flores, F. M., Madejová, J., \& Torres Sánchez, R. M. (2015). Comparison of imazalil removal onto montmorillonite and nanomontmorillonite and adsorption surface sites involved: an approach for agricultural wastewater treatment. Industrial \& Engineering Chemistry Research, 54(5), 1529-1538.

Gast, R.G., (1977). Surface and colloid chemistry. In: Dixon, J.B., Weed, S.B. (Eds.), Minerals in Soil Environments. Soil Science Society of America, Madison, WI, pp. 27-73.

Genena, A. K., Luiz, D. B., Gebhardt, W., Moreira, R. F., José, H. J., \& Schröder, H. F. (2011). Imazalil degradation upon applying ozone-transformation products, kinetics, and toxicity of treated aqueous solutions. Ozone: Science \& Engineering, 33(4), 308-328. 
Glatstein, D. A., \& Francisca, F. M. (2015). Influence of $\mathrm{pH}$ and ionic strength on $\mathrm{Cd}, \mathrm{Cu}$ and $\mathrm{Pb}$ removal from water by adsorption in Na-bentonite. Applied Clay Science, 118, 61-67.

Gök, Ö., Özcan, A. S., \& Özcan, A. (2008). Adsorption kinetics of naphthalene onto organo-sepiolite from aqueous solutions. Desalination, 220(1-3), 96-107.

González López, M.. (2000) Las arcillas como minerales industriales: caolines, bentonitas y arcillas especiales. Discurso de ingreso leido por el academico electo, ilmo. Sr. D. José.

Grevy, J. M., Tellez, F., Bernés, S., Nöth, H., Contreras, R., \& Barba-Behrens, N. (2002). Coordination compounds of thiabendazole with main group and transition metal ions. Inorganica Chimica Acta, 339, 532542.

Groh, M.; Blanche, P.; Calmus, Y.; Guillevin, L. (2012) Thiabendazole-induced acute liver failure requiring transplantation and subsequent diagnosis of polyarteritis nodosa. Clinical and Experimental Rheumatology., 30, S107-S109.

Guimarães, V., Rodríguez-Castellón, E., Algarra, M., Rocha, F., \& Bobos, I. (2016). Influence of pH, layer charge location and crystal thickness distribution on U (VI) sorption onto heterogeneous dioctahedral smectite. Journal of hazardous materials, 317, 246-258.

Guo, T., Cao, S., Su, R., Li, Z., Hu, P., \& Xu, Z. (2011). Adsorptive property of Cu2+-loaded montmorillonite clays for Escherichia coli K88in vitro. Journal of Environmental Sciences, 23(11), 18081815.

Gupta, S. S., \& Bhattacharyya, K. G. (2011). Kinetics of adsorption of metal ions on inorganic materials: a review. Advances in Colloid and Interface Science, 162(1), 39-58.

Hameed, B. H., Salman, J. M., \& Ahmad, A. L. (2009). Adsorption isotherm and kinetic modeling of 2, 4D pesticide on activated carbon derived from date stones. Journal of Hazardous Materials, 163(1), 121-126.

Hazime, R., Ferronato, C., Fine, L., Salvador, A., Jaber, F., \& Chovelon, J. M. (2012). Photocatalytic degradation of imazalil in an aqueous suspension of $\mathrm{TiO}_{2}$ and influence of alcohols on the degradation. Applied Catalysis B: Environmental, 126, 90-99.

Hazime, R., Nguyen, Q. H., Ferronato, C., Salvador, A., Jaber, F., \& Chovelon, J. M. (2014). Comparative study of imazalil degradation in three systems: $\mathrm{UV} / \mathrm{TiO}{ }_{2}, \mathrm{UV} / \mathrm{K}_{2} \mathrm{~S}_{2} \mathrm{O}_{8}$ and $\mathrm{UV} / \mathrm{TiO}_{2} / \mathrm{K}_{2} \mathrm{~S}_{2} \mathrm{O}_{8}$. Applied Catalysis B: Environmental, 144, 286-291. 
He, H. P., Guo, J. G., Xie, X. D., \& Peng, J. L. (2001). Location and migration of cations in Cu 2+adsorbed montmorillonite. Environment International, 26(5), 347-352.

He, H., Frost, R. L., Bostrom, T., Yuan, P., Duong, L., Yang, D., \& Kloprogge, J. T. (2006). Changes in the morphology of organoclays with HDTMA+ surfactant loading. Applied Clay Science, 31(3), 262-271.

Hedley, C. B., Yuan, G., \& Theng, B. (2007). Thermal analysis of montmorillonites modified with quaternary phosphonium and ammonium surfactants. Applied Clay Science, 35(3), 180-188.

Heinz, H., Lin, T.-J., Kishore Mishra, R. \& Emami, F.S., (2013). Thermodynamically Consistent Force Fields for the Assembly of Inorganic, Organic, and Biological Nanostructures: The INTERFACE Force Field. Langmuir 29, 1754-1765.

Heller-Kallai, L., \& Mosser, C. (1995). Migration of $\mathrm{Cu}$ ions in $\mathrm{Cu}$ montmorillonite heated with and without alkali halides. Clays and Clay Minerals, 43(6), 738-743.

Heller-Kallai, L., Bergaya, F., Theng, B. K. G., \& Lagaly, G. (2006). Handbook of Clay Science. Developments in Clay Science.

Hermosin, M. C., Roldán, I., \& Cornejo, J. (1992). Binding mechanisms of maleic hydrazide to homoionic montmorillonites. Science of the total environment, 123, 109-119.

Hjorth K., Johansen K., Holen B., Andersson A., Christensen H.B., Siivinen K. y Toome M. (2011). Pesticide. residues in fruits.and vegetables from South America. A Nordic project. Food Control 22, 1701 1706.

Holešová, S., Kulhánková, L., Martynková, G. S., Kukutschová, J., \& Čapková, P. (2009). An effective route to montmorillonite intercalation with imidazole complexes: Experiment and theory. Journal of Molecular Structure, 923(1), 85-89.

Hu, J. Y., Aizawa, T., Ookubo, Y., Morita, T., \& Magara, Y. (1998). Adsorptive characteristics of ionogenic aromatic pesticides in water on powdered activated carbon. Water Research, 32(9), 2593-2600.

Hunter, R. J. (2013). Zeta potential in colloid science: principles and applications, (Vol. 2). Academic press.

Hyun, S. P., Cho, Y. H., Kim, S. J., \& Hahn, P. S. (2000). Cu (II) sorption mechanism on montmorillonite: an electron paramagnetic resonance study. Journal of colloid and interface science, 222(2), 254-261. 
Idigoras G., (2014), Producción y procesamiento de productos frutihortícolas Documento de referencia, Argentina Innovadora 2020, Plan Nacional de Ciencia, Tecnología e Innovación Productiva, Ministerior de Ciencia, Tecnología e Innovación Productiva de la Nación. http://www.argentinainnovadora2020.mincyt.gob.ar/?wpfb_dl=80

INDEC, 2009. Encuesta Nacional Minera y el Censo Nacional Económico, 2004/2005. http://www.indec.gov.ar/nivel4_default.asp?id_tema_1=3\&id_tema_2=7\&id_tema_3=105

INTA (2016) Productos fitosanitarios para poscosecha de peras y manzanas - Temporada 2015/2016, disponible $\quad$ en: http://inta.gob.ar/sites/default/files/inta_productosfitosanitarios_poscosecha_pera_manzana_2015-2016.pdf

Iñíguez Rodríguez, A.M.. (1972) Estudio Geológico Económico de los yacimientos de bentonitas del lago Pellegrini, Río Negro. Dir. Gral. Min. Río negro. Inédito.

Jaber, M., Komarneni, S., \& Zhou, C. H. (2013). Synthesis of clay minerals. Handbook of Clay Science, 5, 223.

Jaber, M., Miehé-Brendlé, J., Michelin, L., \& Delmotte, L. (2005). Heavy metal retention by organoclays: synthesis, applications, and retention mechanism. Chemistry of materials, 17(21), 5275-5281.

Janisiewicz, W.; Peterson, D.; Yoder, K.; Miller, S. (2005). Experimental bin drenching system for testing biocontrol agents to control postharvest decay of apples. Plant Disease 89: 487-490.

Jasmund, K., Lagaly, G., (1993). Tonminerale und Tone. Struktur, Eigenschaften, Anwendung und Einsatz in Industrie und Umwelt, Steinkopff Verlag, Darmstadt.

Jaynes, W. F., \& Boyd, S. A. (1991). Hydrophobicity of siloxane surfaces in smectites as revealed by aromatic hydrocarbon adsorption from water. Clays and Clay Minerals, 39(4), 428-436.

Jiang, J., Ai, L., \& Li, L. (2009). Synthesis and characterization of polyaniline-based nanocomposites containing magnetic Li-Ni-La ferrite. Journal of Non-Crystalline Solids, 355(34), 1733-1736.

Jiménez-Tototzintle, M., Oller, I., Hernández-Ramírez, A., Malato, S., \& Maldonado, M. I. (2015). Remediation of agro-food industry effluents by biotreatment combined with supported $\mathrm{TiO}_{2} / \mathrm{H}_{2} \mathrm{O}_{2}$ solar photocatalysis. Chemical Engineering Journal, 273, 205-213.

Jin, X., Zha, S., Li, S., \& Chen, Z. (2014). Simultaneous removal of mixed contaminants by organoclaysamoxicillin and $\mathrm{Cu}$ (II) from aqueous solution. Applied Clay Science, 102, 196-201. 
Jing, P., Hou, M., Zhao, P., Tang, X., \& Wan, H. (2013). Adsorption of 2-mercaptobenzothiazole from aqueous solution by organo-bentonite. Journal of Environmental Sciences, 25(6), 1139-1144.

Johnston C.T. (1996), Sorption of organic compunds on clay minerals: A surface functional group approach. Agronomy Departmnt. Crop, Soil and Environmental Sciences 1150 Lilli Hall, Prudue University.

Joseph-Ezra, H., Nasser, A., \& Mingelgrin, U. (2014). Surface interactions of pyrene and phenanthrene on Cu-montmorillonite. Applied Clay Science, 95, 348-356.

Karickhoff, S.W., Bailey, G.W., (1976). Protonation of organic bases in clay-water systems. Clays and Clay Minerals 24, 170-176.

Kaya, A., \& Ören, A. H. (2005). Adsorption of zinc from aqueous solutions to bentonite. Journal of Hazardous Materials, 125(1), 183-189.

Keidar, O., Lapides, I., Shoval, S., \& Yariv, S. (2015). Thermogravimetry and differential thermal analysis of montmorillonite treated with 1, 4-diaminoanthraquinone. Journal of Thermal Analysis and Calorimetry, 120(1), 33-43.

Kinniburgh, D. G. (1986). General purpose adsorption isotherms. Environmental Science \& Technology, 20(9), 895-904.

Kolthoff I. M. \& Sander E.B. (1948) Textbook of Quantitative Inorganic Analysis. 3 Ed. Cap. VI, pp. 72-105. Macmillan,

Kolthoff, I. M., \& Sandell, E. B. (1943). Textbook of quantitative inorganic analysis.

Koswojo, R., Utomo, R. P., Ju, Y. H., Ayucitra, A., Soetaredjo, F. E., Sunarso, J., \& Ismadji, S. (2010). Acid Green 25 removal from wastewater by organo-bentonite from Pacitan. Applied clay science, 48(1), 8186.

Kowala, C., Murray, K. S., Swan, J. M., \& West, B. O. (1971). Transition metal complexes of thiabendazole. Australian Journal of Chemistry, 24(7), 1369-1375.

Kowalska, M.; Guler, H.; Cocke, D. L. (1994) Interactions of clay minerals with organic pollutants. Sci. Total Environ., 141, 223-240.

Ladd, M.; Palmer, R. (2003) Structure determination by X-ray crystallography. Kluwer Academic. New York. USA. 
Lagadic, I.L., Mitchell, M.K., Payne, B.D., (2001). Highly effective adsorption of heavy metal ions by a thiol-functionalized magnesium phyllosilicate clay. Environmental Science \& Technology 35, 984-990.

Lagaly, G., \& Dékany, I. (2005). Adsorption on hydrophobized surfaces: clusters and selforganization. Advances in Colloid and Interface Science, 114, 189-204.

Lagaly, G., (1984). Clay-organic interactions. Philosophical Transactions of the Royal Society of London 311, 315-332.

Lázaro-Martínez, J. M., Monti, G. A., \& Chattah, A. K. (2013). Insights into the coordination sphere of copper ion in polymers containing carboxylic acid and azole groups. Polymer, 54(19), 5214-5221.

Lázaro-Martínez, J. M., Rodríguez-Castellón, E., Vega, D., Monti, G. A., \& Chattah, A. K. (2015). Solidstate Studies of the Crystalline/Amorphous Character in Linear Poly (ethylenimine hydrochloride)(PEI $\cdot \mathrm{HCl}$ ) Polymers and Their Copper Complexes. Macromolecules, 48(4), 1115-1125.

Leach, A. R. (2001). Molecular modelling: principles and applications. Pearson education.

Lee, S. M., \& Tiwari, D. (2012). Organo and inorgano-organo-modified clays in the remediation of aqueous solutions: an overview. Applied Clay Science, 59, 84-102.

Lee, S.Y., Cho, W.J., Hahn, P.S., Lee, M., Lee, Y.B., \& Kim, K.J., (2005). Microstructural changes of reference montmorillonites by cationic surfactants. Appl. Clay Sci. 30, 174-180.

Lelario, F., Gardi, I., Mishael, Y., Dolev, N., Undabeytia, T., Nir, S., \& Bufo, S. A. (2016). Pairing micropollutants and clay-composite sorbents for efficient water treatment: Filtration and modeling at a pilot scale. Applied Clay Science.

Li, S., Leroy, P., Heberling, F., Devau, N., Jougnot, D., Chiaberge, C. (2016) Influence of surface conductivity on the apparent zeta potential of calcite Journal of Colloid and Interface Science, 468, pp. 262275.

Li, Y.; Ishida, H. (2003) Concentration-dependent conformation of alkyl tail in the nanoconfined space: Hexadecylamine in the silicate galleries. Langmuir, 19, 2479-2484.

Li, Z.-Y., Xu, R.-K., Li, J.-Y., Hong, Z.-N. (2016) Effect of clay colloids on the zeta potential of Fe/Al oxide-coated quartz: a streaming potential study Journal of Soils and Sediments, pp. 1-11.

Lifshin, E. (1999). X-Ray Characterization of materials. Wiley. VCH. 
Liu, R., Frost, R.L., Martens, W.N., Yuan, Y., (2008). Synthesis, characterization of mono, di and tri alkyl surfactant intercalated Wyoming montmorillonite for the removal of phenol from aqueous systems. Jorunal of Colloid Interface Science, 327, 287-294.

Lombardi, B., Baschini, M., \& Sánchez, R. T. (2003). Optimization of parameters and adsorption mechanism of thiabendazole fungicide by a montmorillonite of North Patagonia, Argentina. Applied clay science, $24(1)$, 43-50.

Lopez, F. Espectroscopía de Resonancia Magnética, disponible en: https://www.uam.es/personal_pdi/ciencias/rega/descargas_docentes/Espectroscopia\%20de\%20Resonancia $\% 20$ Magnetica.pdf

Lützenkirchen, J., Abdelmonem, A., Weerasooriya, R., Heberling, F., Metz, V., Marsac, R. (2014) Adsorption of dissolved aluminum on sapphire-c and kaolinite: Implications for points of zero charge of clay minerals Geochemical Transactions, 15 (1), art. no. 9.

Ma, L., Chen, Q., Zhu, J., Xi, Y., He, H., Zhu, R., \& Ayoko, G. A. (2016). Adsorption of phenol and Cu (II) onto cationic and zwitterionic surfactant modified montmorillonite in single and binary systems. Chemical Engineering Journal, 283, 880-888.

Ma, Y. L., Xu, Z. R., Guo, T., \& You, P. (2004). Adsorption of methylene blue on Cu (II)-exchanged montmorillonite. Journal of Colloid and Interface Science, 280(2), 283-288.

Mackenzie, R.C. (1970). Differential Thermal analyses. Fundamental aspects. Vol. I \& II. Academic Press. London \& New York.

Madejová, J., \& Komadel, P. (2001). Baseline studies of the clay minerals society source clays: infrared methods. Clays and clay minerals, 49(5), 410-432.

Madejová, J., Arvaiová, B., \& Komadel, P. (1999). FTIR spectroscopic characterization of thermally treated $\mathrm{Cu} 2+, \mathrm{Cd} 2+$, and $\mathrm{Li}+$ montmorillonites. Spectrochimica Acta Part A: Molecular and Biomolecular Spectroscopy, 55(12), 2467-2476.

Madejová, J., Jankovič, L., Pentrák, M., \& Komadel, P. (2011). Benefits of near-infrared spectroscopy for characterization of selected organo-montmorillonites. Vibrational Spectroscopy, 57(1), 8-14.

Madejová, J., Pálková, H., \& Komadel, P. (2006). Behaviour of Li+ and Cu 2+ in heated montmorillonite: Evidence from far-, mid-, and near-IR regions. Vibrational Spectroscopy, 40(1), 80-88. 
Magnoli, A. P., Tallone, L., Rosa, C. A., Dalcero, A. M., Chiacchiera, S. M., \& Sanchez, R. M. T. (2008). Commercial bentonites as detoxifier of broiler feed contaminated with aflatoxin. Applied Clay Science, 40(1), 63-71.

Malamis, S., \& Katsou, E. (2013). A review on zinc and nickel adsorption on natural and modified zeolite, bentonite and vermiculite: Examination of process parameters, kinetics and isotherms. Journal of hazardous materials, 252, 428-461.

Maltas, E., \& Ozmen, M. (2015). Spectrofluorometric and thermal gravimetric study on binding interaction of thiabendazole with hemoglobin on epoxy-functionalized magnetic nanoparticles. Materials Science and Engineering: C, 54, 43-49.

Marco Brown, J. L. (2011) Tesis para obtener el titulo de Doctor de la Universidad de Buenos Aires, tema: Adsorción de de Picloran sobre minerales arcillosos. FECN- UBA.

Marco-Brown, J. L., Areco, M. M., Sánchez, R. M. T., \& dos Santos Afonso, M. (2014). Adsorption of picloram herbicide on montmorillonite: kinetic and equilibrium studies. Colloids and Surfaces A: Physicochemical and Engineering Aspects, 449, 121-128.

Markarian, J. (2005). Automotive and packaging offer growth opportunities for nanocomposites. Plastics, Additives and Compounding, 7(6), 18-21.

Martín-González, M. A., González-Díaz, O., Susial, P., Araña, J., Herrera-Melián, J. A., Doña-Rodríguez, J. M., \& Pérez-Peña, J. (2014). Reuse of Phoenix canariensis palm frond mulch as biosorbent and as precursor of activated carbons for the adsorption of Imazalil in aqueous phase. Chemical Engineering Journal, 245, 348-358.

Masiá, A.; Campo, J.; Navarro-Ortega, A.; Barceló, D.; Picó, Y. (2014) Pesticide monitoring in the basin of Llobregat River (Catalonia, Spain) and comparison with historical data. Sciience of the total Environment., $503-504,58-68$.

Mercier, L., Detellier, C., (1995). Preparation, characterization and application as heavy metal sorbents of covalently grafted thiol functionalities on the interlamellar surface of montmorillonite. Environmental Science \& Technology 29, 1318-1323.

Morton, J. D., Semrau, J. D., \& Hayes, K. F. (2001). An X-ray absorption spectroscopy study of the structure and reversibility of copper adsorbed to montmorillonite clay. Geochimica et Cosmochimica Acta, 65(16), 2709-2722. 
Mothilal, K. K., Karunakaran, C., Rao, P. S., \& Murugesan, R. (2003). Single crystal EPR of Cu (II) doped [Co (tbz) 2 (NO 3)(H 2 O)] NO 3: probe into copper-thiabendazole interaction. Spectrochimica Acta Part A: Molecular and Biomolecular Spectroscopy, 59(14), 3337-3345.

Naranjo, P.M., Sham, E.L., Castellón, E.R., Torres Sánchez, R.M., \& Farfán Torres, E.M., (2013). Identification and quantification of the interaction mechanisms between the cationic surfactant HDTMA-Br and montmorillonite. Clays \& Clay Minerals. 61, 98-106.

Narine D.R., Guy R.D. (1981) Interactions of some large organic cations with bentonite in dilute aqueous systems. Clays and Clays Minerals, Vol. 29, No. 3, 205-212, 1981.

Neumann, M. G., Gessner, F., Schmitt, C. C., \& Sartori, R. (2002). Influence of the layer charge and clay particle size on the interactions between the cationic dye methylene blue and clays in an aqueous suspension. Journal of Colloid and Interface Science, 255(2), 254-259

Nzihou, A., \& Stanmore, B. (2013). The fate of heavy metals during combustion and gasification of contaminated biomass — a brief review. Journal of hazardous materials, 256, 56-66.

Omirou, M., Dalias, P., Costa, C., Papastefanou, C., Dados, A., Ehaliotis, C., \& Karpouzas, D. G. (2012). Exploring the potential of biobeds for the depuration of pesticide-contaminated wastewaters from the citrus production chain: Laboratory, column and field studies. Environmental Pollution, 166, 31-39.

OMS, Organización Mundial de la Salud, (2011), Directrices sobre la calidad del agua potable, 4ta Ed., Ginebra.

Osman, M. A., Ploetze, M., \& Skrabal, P. (2004). Structure and properties of alkylammonium monolayers self-assembled on montmorillonite platelets. The Journal of Physical Chemistry B, 108(8), 2580-2588.

Ouellet-Plamondon, C. M., Stasiak, J., \& Al-Tabbaa, A. (2014). The effect of cationic, non-ionic and amphiphilic surfactants on the intercalation of bentonite. Colloids and Surfaces A: Physicochemical and Engineering Aspects, 444, 330-337.

Ouellet-Plamondon, C., Lynch, R. J., \& Al-Tabbaa, A. (2012). Comparison between granular pillared, organo-and inorgano-organo-bentonites for hydrocarbon and metal ion adsorption. Applied Clay Science, 67, 91-98.

Oyanedel-Craver, V. A., \& Smith, J. A. (2006). Effect of quaternary ammonium cation loading and $\mathrm{pH}$ on heavy metal sorption to Ca bentonite and two organobentonites. Journal of Hazardous Materials, 137(2), 1102-1114. 
Özacar, M., \& Şengil, İ. A. (2005). A kinetic study of metal complex dye sorption onto pine sawdust. Process Biochemistry, 40(2), 565-572.

Park, Y., Ayoko, G. A., \& Frost, R. L. (2011). Application of organoclays for the adsorption of recalcitrant organic molecules from aqueous media. Journal of colloid and interface science, 354(1), 292-305.

Park, Y., Ayoko, G. A., Kurdi, R., Horváth, E., Kristóf, J., \& Frost, R. L. (2013). Adsorption of phenolic compounds by organoclays: Implications for the removal of organic pollutants from aqueous media. Journal of colloid and interface science, 406, 196-208.
Pearson
R.
G
(1998)
disponible
en:

http://depa.fquim.unam.mx/amyd/archivero/AcidosDurosyBlandos2_31310.pdf

Pearson, R. G. (1963). Hard and soft acids and bases. Journal of the American Chemical Society, 85(22), 3533-3539.

Pecini, E. M., \& Avena, M. J. (2013). Measuring the isoelectric point of the edges of clay mineral particles: The case of montmorillonite. Langmuir, 29(48), 14926-14934.

Pérez-Marín, A. B., Zapata, V. M., Ortuno, J. F., Aguilar, M., Sáez, J., \& Lloréns, M. (2007). Removal of cadmium from aqueous solutions by adsorption onto orange waste. Journal of hazardous materials, 139(1), 122-131.

Pinnavaia, T.J., Hall, P.L., Cady, S.S., Mortland, M.M., (1974). Aromatic radical cation formation on the intracrystal surfaces of transition metal layer lattice silicates. The Journal of Physical Chemistry 78, 994-999.

Pinnavaia, T.J., Mortland, M.M., (1971). Interlamellar metal complexes of layer silicates. I. Copper(II)arene complexes on montmorillonite. The Journal of Physical Chemistry 75, 3957-3962.

Pinzón-Bedoya, M. L., \& Vera Villamizar, L. E. (2009). Kinetc modeling biosorption of Cr (III) using orange shell. Dyna, 76(160), 95-106.

Plimpton, S. (1995). Fast parallel algorithms for short-range molecular dynamics. Journal of computational physics, 117(1), 1-19.

Pórfido O. D., (2014) Los Plaguicidas en la República Argentina; 1a ed. - Buenos Aires : Ministerio de Salud de la Nación. ISBN 978-950-38-0160-4.

PPDB, Pesticide Properties Data Base (2011) University of Hertfordshire: Hatfield, Hertfordshire, UK, disponible en: http://sitem.herts.ac.uk/aeru/footprint/index2.htm. 
Price, R.J.; Scott, M.P.; Walters, D.G.; Stierum, R.H.; Groten, J.P.; Meredith, C.; Lake, B.G (2004). Effect of thiabendazole on some rat hepatic xenobiotic metabolising enzymes. Food and Chemical Toxicology., 42, 899-908.

Resolución 335/02. Uso del agua en establecimientos de empaque y frigorificos de fruta. Codigo de Aguas Ley Q 2952, Libro III. Río Negro.

Richerson D.W. (1992). Modern Ceramic Engineering - Second Edition, Revised and Expanded (ed. P. Hilton), Marcel Dekker Inc./NY.

Rizzi, A. C., Neuman, N. I., González, P. J., \& Brondino, C. D. (2016). EPR as a Tool for Study of Isolated and Coupled Paramagnetic Centers in Coordination Compounds and Macromolecules of Biological Interest. European Journal of Inorganic Chemistry, 2016(2), 192-207.

Roca Jalil, M. E.; Baschini, M.; Rodríguez-Castellón, E.; Infantes-Molina, A.; Sapag, K. (2014) Effect of the $\mathrm{Al} /$ clay ratio on the thiabendazol removal by aluminum pillared clays. Applied Clay Science, 87, 245-253.

Rong, M., Muir, M. M., Cadiz, M. E., \& Muir, J. A. (1991). Structure of a platinum (II) complex with a ligand containing thiazole and benzimidazole. Acta Crystallographica Section C: Crystal Structure Communications, 47(7), 1539-1541.

Rosenberg, D.A. (2010). Low-volume prestorage drenching is attractive. Revista Good Fruit Grower.

Rupert, J.P., (1973). Electron spin resonance spectra of interlamellar copper(II)-arene complexes on montmorillonite. The Journal of Physical Chemistry 77, 784-790.

Sączewski, F., Dziemidowicz-Borys, E., Bednarski, P. J., Grünert, R., Gdaniec, M., \& Tabin, P. (2006). Synthesis, crystal structure and biological activities of copper (II) complexes with chelating bidentate 2substituted benzimidazole ligands. Journal of inorganic biochemistry, 100(8), 1389-1398.

Saha, A., Tp, A. S., Gajbhiye, V. T., Gupta, S., \& Kumar, R. (2013). Removal of mixed pesticides from aqueous solutions using organoclays: evaluation of equilibrium and kinetic model. Bulletin of environmental contamination and toxicology, 91(1), 111-116.

Sánchez, R. T., Okumura, M., \& Mercader, R. C. (2001). Charge properties of red Argentine soils as an indicator of iron oxide/clay associations. Soil Research, 39(2), 423-434.

Santiago, D. E., Doña-Rodríguez, J. M., Araña, J., Fernández-Rodríguez, C., González-Díaz, O., PérezPeña, J., \& Silva, A. M. (2013). Optimization of the degradation of imazalil by photocatalysis: comparison between commercial and lab-made photocatalysts. Applied Catalysis B: Environmental, 138, 391-400. 
Santiago, D. E., Espino-Estévez, M. R., González, G. V., Araña, J., González-Díaz, O., \& DoñaRodriguez, J. M. (2015). Photocatalytic treatment of water containing imazalil using an immobilized $\mathrm{TiO}_{2}$ photoreactor. Applied Catalysis A: General, 498, 1-9.

SEGEMAR, Anales (2004). Disponible en: http://segemar-intemin-biblioteca.blogspot.com.ar/

Sempere, Emili (2006). Orígenes de la vida y mitología cerámica». Historia y Arte en la cerámica de España y Portugal. Barcelona: Les Punxes. ISBN 978-84-611-3612-4.

SENASA, Servicio Nacional de Sanidad y Calidad Agroalimentaria, Res. No 256/03.

SENASA, 2014, Cítricos argentinos de excelencia, Informe Estadístico para la Coordinación General de Desarrollo y Planificación Estratégica, Ministerio de Agricutlrua, Ganadería y PescA, http://www.senasa.gov.ar/sites/default/files/ARBOL_SENASA/INFORMACION/INFORMES $\% 20 Y \% 2$ 0ESTADISTICAS/Informes $\% 20 \mathrm{y} \% 20$ estadisticas\%20Vegetal/FRUTALES/citricos_argentinos_de_excelen cia.pdf.

Sethuraman, V., Stanley, N., Thomas Muthiah, P., \& Karunakaran, C. (2002). Supramolecular selfassembly via inter-ligands hydrogen bonds in $[\mathrm{Cu}(\mathrm{H} 2 \mathrm{O}) 2$ (NO3) 2 (tb)](tb is thiabendazole). Acta Crystallographica Section E: Structure Reports Online, 58(8), m392-m395.

Seyama, H., \& Soma, M. (1985). Bonding-state characterization of the constitutent elements of silicate minerals by X-ray photoelectron spectroscopy. Journal of the Chemical Society, Faraday Transactions 1: Physical Chemistry in Condensed Phases, 81(2), 485-495.

Sheng, G., Xu, S.H., Boyd, S.A., (1999). A dualfunction organoclay sorbent for lead and chlorobenzene. Soil Science Society of America Journal 63, 73-78.

Shmuel Yariv, The role of charcoal on DTA curves of organo-clay complexes: an overview, Applied Clay Science 24 (2004) 225- 236

Skoog, D. A. (2001) Principios de Análisis Instrumental. McGrawHill.

Socias-Viciana, M. M., Hermosin, M. C., \& Cornejo, J. (1998). Removing prometrone from water by clays and organic clays. Chemosphere, 37(2), 289-300.

Souza Casadinho J. (2008). Alternativas al endosulfán en la soja: El caso de Argentina. En “El endosulfán y sus alternativas en América Latina. Oficina para América Latina y el Caribe del Pesticide Action Network (PAN) Internacional disponible en: http://www.rapaluruguay.org/ endosulfan/ Alternativas_endosulfan pdf $27 / 4 / 2013$. 
Souza Casadinho J. (2009). La problemática del uso de plaguicidas en argentina. Modelos productivos e impacto en el ambiente. Red de Acción en Plaguicidas y sus Alternativas de América latina RAPAL, disponible en: http://www.rap-al.org/articulos_files/Plaguicidas_Argentina.pdf 15/4/2013.

Souza Casadinho J., Weber J.M., Guadarrama Zugasti C., Escamilla Prado E., Beristáin Ruiz B., Acosta M., Cárcamo M. I., Ramírez Muñoz F. (2008). El endosulfán y sus alternativas en América Latina, disponible en: http:// www.rapaluruguay.org/ endosulfan/Alternativas_endosulfan.pdf. 27/4/2013.

Stathi, P., Litina, K., Gournis, D., Giannopoulos, T.S., Deligiannakis, Y., (2007). Physicochemical study of novel organoclays as heavy metal ion adsorbents for environmental remediation. Journal of Colloid and Interface Science 316, 298-309.

Suárez Pérez, Y., Izquierdo Castro, A., \& Milián Sánchez, J. M. (2009). Validación de un método cromatográfico para la cuantificación de mefenesina en tabletas de producción nacional. Revista Cubana de Farmacia, 43(2), 0-0. Disponible en: http://www.bvs.sld.cu/revistas/far/vol43_2_09/far05209.htm

Szabo, A., Gournis, D., Karakassides, M.A., Petridis, D., (1998). Clay-aminopropylsiloxane compositions. Chemistry of Materials 10, 639-645.

Tabak, A., Afsin, B., Aygun, S. F., \& Koksal, E. (2006). Structural characteristics of organo-modified bentonites of different origin. Journal of thermal analysis and calorimetry, 87(2), 377-382.

Tada, Y.; Fujitani, T.; Yano, N.; Yuzawa, K.; Nagasawa, A.; Yoneyama; M. (2001) Thiabendazole induces urinary tract toxicity in male ICR mice. Toxicology., 162, 1-10.

Tada, Y.; Fujitani, T.; Yoneyama, M. (1992) Acute renal toxicity of thiabendazole (TBZ) in ICR mice. Food and Chemical Toxicology. 30, 1021-1030.

Theng, B. K. G. (1974). The Chemistry of Clay-Organic Reactions. The Chemistry of Clay-Organic Reactions.

Thomas, F., Michot, L. J., Vantelon, D., Montarges, E., Prelot, B., Cruchaudet, M., \& Delon, J. F. (1999). Layer charge and electrophoretic mobility of smectites. Colloids and Surfaces A: Physicochemical and Engineering Aspects, 159(2), 351-358.

Tong, D. S., Zhou, C. H. C., Lu, Y., Yu, H., Zhang, G. F., \& Yu, W. H. (2010). Adsorption of acid red G dye on octadecyl trimethylammonium montmorillonite. Applied Clay Science, 50(3), 427-431. 
Tonle, I.K., Ngameni, E., Njopwouo, D., Carteret, C., Walcarius, A., (2003). Functionalization of natural smectite-type clays by grafting with organosilanes: physicochemical characterization and application to mercury(II) uptake. Physical Chemistry Chemical Physics 5, 4951-4961.

Torres Sánchez, R. M., Genet, M. J., Gaigneaux, E. M., dos Santos Afonso, M., Yunes, S., (2011). Benzimidazole adsorption on the external and interlayer surfaces of raw and treated montmorillonite. Applied Clay Science. 53, 366-373.

Tsai, W. C., \& Lin, J. J. (2010). Copper-Ion-Assisted Self-Assembly of Silicate Clays in Rod-and Disklike Morphologies. Langmuir, 26(12), 10177-10182.

Tschapek, M., Sanchez, R. M., \& Wasowski, C. (1989). Handy methods for determining the isoelectric point of soils. Journal of Plant Nutrition and Soil Science, 152(1), 73-76.

Tsipursky, S. J. \& Drits, V. A., (1984). The distribution of octahedral cations in 2:1 layers of dioctahedral smectites studied by oblique-texture electron diffraction. Clay Minerals 19, 177-193.

Uclés, A., López, S. H., Hernando, M. D., Rosal, R., Ferrer, C., \& Fernández-Alba, A. R. (2015). Application of zirconium dioxide nanoparticle sorbent for the clean-up step in post-harvest pesticide residue analysis. Talanta, 144, 51-61.

Union Europea, COUNCIL DIRECTIVE 98/83/EC (1998) disponible en: http://eurlex.europa.eu/LexUriServ/LexUriServ.do?uri=OJ:L:1998:330:0032:0054:EN:PDF

Vazquez, A.; López, M.; Kortaberria, G.; Martín, L.; Mondragon, I. (2008) Modification of montmorillonite with cationic surfactants. Thermal and chemical analysis including CEC determination. Applied Clay Science, 41, 24-36.

Villaamil Lepori E.C, Bovi Mitre G. y Nassetta M.., Situación actual de la contaminación por plaguicidas en argentina, Revista Internacional de Contaminación Ambiental, 29 (Número especial sobre plaguicidas), pp $25-43$.

Wang, G., Su, X., Hua, Y., Ma, S., Wang, J., Xue, X., \& Komarneni, S. (2016). Kinetics and thermodynamic analysis of the adsorption of hydroxy-Al cations by montmorillonite. Applied Clay Science, 129, 79-87.

Weiss, A. (1963). Organische derivate der glimmerartigen Schichtsilicate. Angewandte Chemie, 75(2), 113122.

Wellmann M. (1999) Pedanii Dioscuridis Anazarbei, I, II y III, Berlín pp.1906-1914. 
Wicklein, B., Darder, M., Aranda, P., \& Ruiz-Hitzky, E., (2010). Bio-organoclays based on phospholipids as immobilization hosts for biological species. Langmuir 26, 5217-5225.

Workman Jr, J., \& Weyer, L. (2007). Practical guide to interpretive near-infrared spectroscopy. CRC press.

Wu, Y., Zhang, L., Gao, C., Ma, J., Ma, X., \& Han, R. (2009). Adsorption of copper ions and methylene blue in a single and binary system on wheat straw. Journal of Chemical \& Engineering Data, 54(12), 32293234.

Xi, Y. (2006). Synthesis, characterization and application of organoclays. Thesis Doctor of Philosophy. Queensland University of Technology. School of Physical and Chemical Sciences. Inorganic Material Research Group.

Xi, Y., Frost, R.L., He, H., (2007). Modification of the surfaces of Wyoming montmorillonite by the cationic surfactants alkyl trimethyl, dialkyl dimethyl, and trialkyl methyl ammonium bromides. Jorunal of Colloid Interface Science 305, 150-158.

Xu, S., \& Boyd, S. A. (1995). Cationic surfactant adsorption by swelling and nonswelling layer silicates. Langmuir, 11(7), 2508-2514.

Xu, W. J., Xue, Q. J., Liang, P., Zhang, L. Y., Huang, Y. F., \& Feng, Y. (2014). A Series of Transitionmetal Coordination Complexes Assembled from 3-Nitrophthalic Acid and Thiabendazole: Synthesis, Structure and Properties. Bulletin of the Korean Chemical Society, 35(1), 218-224.

Yariv, S. (2001) IR spectroscopy and Thermo-IR spectroscopy in the study of the fine structure of organo-clay complexes. In Organo-Clay Complexes and Interactions; Yariv, S.; Cross, H., Eds.; Marcel Dekker, Inc: New York, pp 345-462.

Yariv, S., Cross, H. (Eds.), (2002). Organo-Clay Complexes and Interactions. Marcel Dekker, New York.

Yoshioka, N., Akiyama, Y., \& Teranishi, K. (2004). Rapid simultaneous determination of o-phenylphenol, diphenyl, thiabendazole, imazalil and its major metabolite in citrus fruits by liquid chromatography-mass spectrometry using atmospheric pressure photoionization. Journal of Chromatography A, 1022(1), 145-150.

Zhang, Y., Zhao, Y., Zhu, Y., Wu, H., Wang, H., \& Lu, W. (2012). Adsorption of mixed cationic-nonionic surfactant and its effect on bentonite structure. Journal of Environmental Sciences, 24(8), 1525-1532.

Zhu, H., Njuguna, J. Nanolayered silicates/clay minerals: Uses and effects on health (2014). IN: Health and Environmental Safety of Nanomaterials: Polymer Nancomposites and Other Materials Containing Nanoparticles, pp. 133-146. 
Zhu, J., He, H., Guo, J., Yang, D., \& Xie, X. (2003). Arrangement models of alkylammonium cations in the interlayer of HDTMA+ pillared montmorillonites. Chinese Science Bulletin, 48(4), 368-372.

Zhu, R., Chen, Q., Zhu, R., Xu, Y., Ge, F., Zhu, J., \& He, H. (2015). Sequestration of heavy metal cations on montmorillonite by thermal treatment. Applied Clay Science, 107, 90-97. 


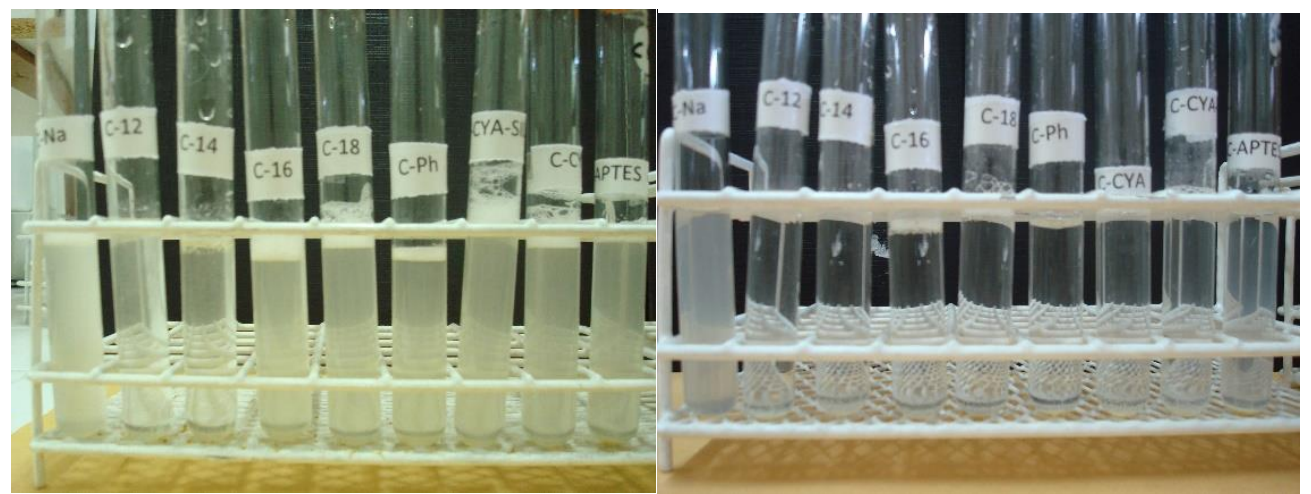

Figura A1. Foto de la suspensión de montmorillonita y de organo-montmorillonitas en agua. A la izquierda $t$ $=0$, y a la derecha, $t=24 \mathrm{~h}$. El primer tubo desde la izquierda corresponde a la muestra Mt; y el quinto a la muestra OMt137.
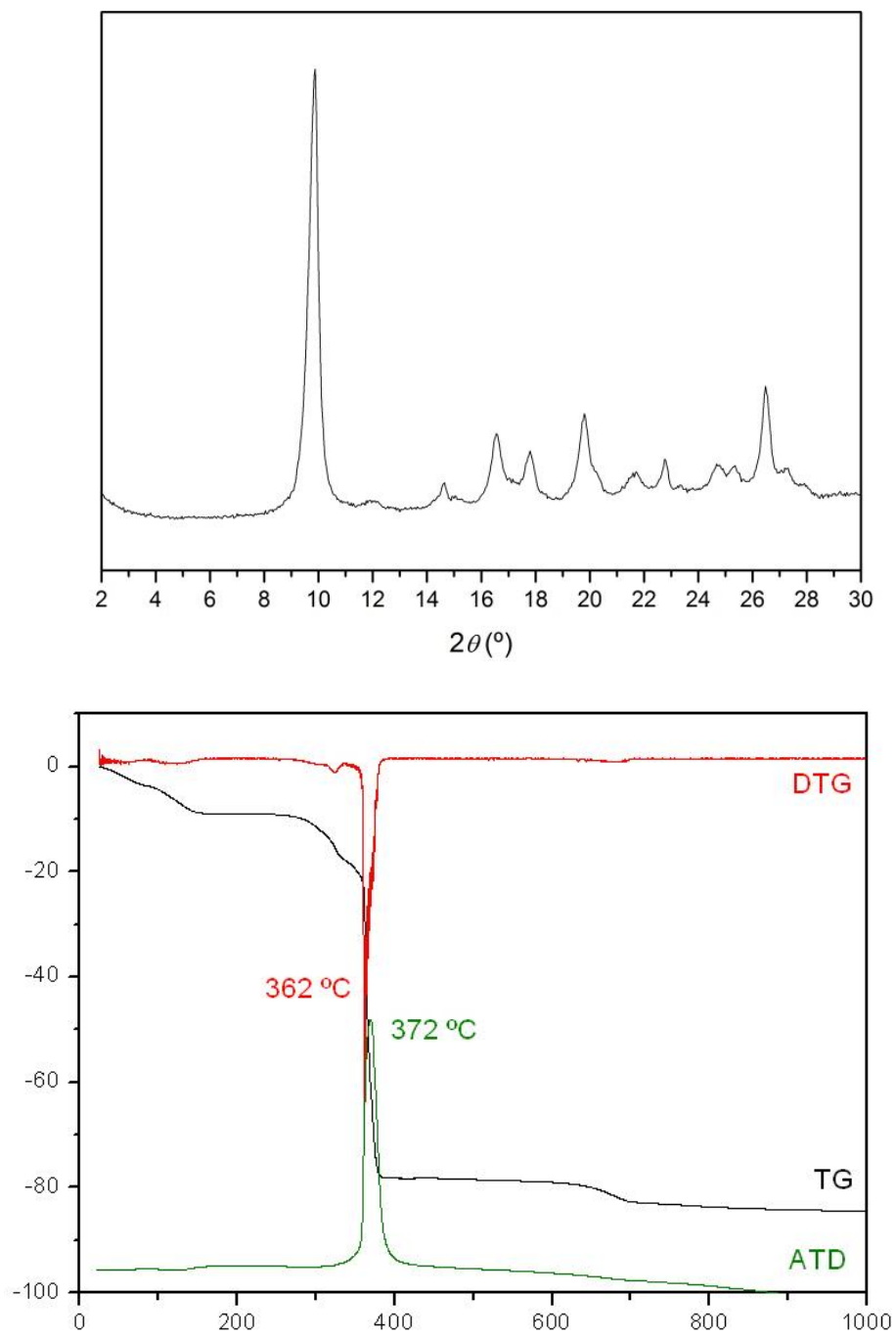

Figura A2. DRX y análisis térmicos del complejo Cu-TBZ. 
Tabla A1. Número de onda $\left[\mathrm{cm}^{-1}\right]$ y asignación de las bandas observadas en el espectro MIR de las muestras indicadas

\begin{tabular}{|c|c|c|c|c|c|c|c|c|c|}
\hline $\mathrm{Mt}$ & OMt39 & OMt55 & OMt75 & OMt137 & IMZ & IMZ-Mt & IMZ-OMt39 & IMZ-OMt55 & Asignación \\
\hline 3631 & 3633 & 3631 & 3633 & 3627 & & 3628 & 3628 & 3628 & $v(\mathrm{OH})_{\mathrm{m}}$ \\
\hline \multirow[t]{8}{*}{3421} & 3402 & 3395 & 3400 & 3395 & 3422 & 3367 & 3422 & 3401 & $v(\mathrm{OH})_{\mathrm{w}}$ \\
\hline & & & & & & 3156 & 3155 & 3155 & $v \mathrm{~N}-\mathrm{H}^{+}$ \\
\hline & & & & 3009 & & & & & $v \mathrm{CH}_{3}-\mathrm{N}$ \\
\hline & & & 2925 & 2919 & 3093 & 3089 & 3087 & 3088 & $v \mathrm{C}-\mathrm{H}(\mathrm{Ar}), v=\mathrm{CH}_{2}$ \\
\hline & 2927 & 2927 & 2853 & 2850 & 2927 & 2928 & 2925 & 2925 & $v_{\mathrm{as}} \mathrm{CH}_{2}$ \\
\hline & 2855 & 2854 & & & 2860 & 2871 & 2854 & 2854 & $v_{\mathrm{s}} \mathrm{CH}_{2}$ \\
\hline & & & & & & 1730 & 1730 & 1730 & $v \mathrm{C}=\mathrm{O}$ \\
\hline & & & & & 1647 & & & & $v \mathrm{C}=\mathrm{CH}_{2}$ \\
\hline \multirow[t]{10}{*}{1629} & 1630 & 1626 & 1627 & 1622 & & & & & $\delta(\mathrm{OH})_{\mathrm{w}}$ \\
\hline & & & & & 1589 & 1590 & 1590 & 1590 & $v \mathrm{C}=\mathrm{C}(\mathrm{Ar})$ \\
\hline & & & & & 1561 & 1563 & 1562 & 1562 & $v \mathrm{C}=\mathrm{C}(\mathrm{Ar})$ \\
\hline & & & & & & 1550 & & & $\delta \mathrm{N}-\mathrm{H}^{+}$ \\
\hline & & & & & 1505 & 1509 & 1510 & 1507 & $v \mathrm{C}=\mathrm{C}(\mathrm{Ar})$ \\
\hline & 1488 & 1488 & 1488 & 1488 & & & & & $\delta_{\text {as }} \mathrm{CH}_{3}-\mathrm{N}$ \\
\hline & 1473 & 1475 & 1475 & 1469 & 1470 & 1471 & 1470 & 1470 & $v \mathrm{C}=\mathrm{C}(\mathrm{Ar}), v \mathrm{C}-\mathrm{N}$ \\
\hline & & & & & & & & & $\mathrm{CH}_{2}$ \\
\hline & & & 1417 & 1418 & & & & & $\delta_{\mathrm{as}} \mathrm{C}-\mathrm{H}$ \\
\hline & & & & 1383 & 1382 & 1380 & 1381 & 1380 & $\nu \mathrm{C}=\mathrm{C}(\mathrm{Ar}), \delta_{\mathrm{s}} \mathrm{C}-\mathrm{H}$ \\
\hline
\end{tabular}




\begin{tabular}{|c|c|c|c|c|c|c|c|c|c|}
\hline & & & & & 1340 & 1345 & 1348 & 1348 & $\delta \mathrm{CH}(\mathrm{Ar})$ \\
\hline & & & & & 1285 & 1289 & 1287 & 1286 & $v \mathrm{C}=\mathrm{C}(\mathrm{Ar}), \delta \mathrm{C}-\mathrm{H}$ \\
\hline 1043 & 1046 & 1047 & 1047 & 1044 & & 1046 & 1046 & 1045 & $v \mathrm{Si}-\mathrm{O}$ \\
\hline 917 & 918 & 917 & 916 & 914 & & 918 & 918 & 917 & $\delta \mathrm{AlAlOH}$ \\
\hline 884 & 884 & 885 & 884 & 887 & & 885 & 886 & 886 & $\delta \mathrm{AlFeOH}$ \\
\hline 844 & 847 & 846 & 844 & 843 & & 840 & 841 & 841 & $\delta \mathrm{AlMgOH}$ \\
\hline \multirow[t]{3}{*}{798} & 798 & 797 & 796 & 797 & & 790 & 792 & 791 & $v \mathrm{Si}-\mathrm{O}$ \\
\hline & & 721 & 721 & 721 & & & 728 & 727 & rocking $\mathrm{CH}_{2}$ \\
\hline & & & 623 & 624 & 662 & & & 661 & $\delta_{\text {fuera del plano }} \mathrm{CH}(\mathrm{Ar})$ \\
\hline \multirow[t]{2}{*}{523} & 523 & 522 & 521 & 521 & & 521 & 521 & 521 & $\delta A 1 O S i$ \\
\hline & 465 & 465 & 464 & 464 & & 464 & 464 & 464 & $\delta \mathrm{SiOSi}$ \\
\hline
\end{tabular}

s: simétrico; as: antisimétrico 
Tabla A2. Número de onda $\left[\mathrm{cm}^{-1}\right]$ y asignación de las bandas observadas en el espectro NIR de las muestras indicadas.

\begin{tabular}{|c|c|c|c|c|c|c|c|c|c|}
\hline $\mathrm{Mt}$ & OMt39 & OMt55 & OMt75 & OMt137 & IMZ & IMZ-Mt & IMZ-OMt39 & IMZ-OMt55 & Asignación \\
\hline & & & & & & 7170 & 7170 & 7170 & $2 v \mathrm{OH}$ \\
\hline 7080 & 7080 & 7080 & 7079 & 7077 & & 7080 & 7078 & 7078 & $2 v(\mathrm{OH})_{\mathrm{m}}, 2 v(\mathrm{OH})_{\mathrm{w}}$ \\
\hline \multirow[t]{7}{*}{6800} & 6800 & 6800 & & & & & & & $2 v(\mathrm{OH})_{\mathrm{m}}$ \\
\hline & & & & & & 6200 & & & $2 v \mathrm{NH}^{+}$ \\
\hline & & & & & 6120 & 6137 & 6131 & 6133 & $2 v \mathrm{CH}_{2}=\mathrm{C}$ \\
\hline & 6012 & 6006 & 6002 & 5999 & 5991 & 6003 & 6003 & 6006 & $2 v \mathrm{CH}_{3}-\mathrm{N}, 2 v \mathrm{C}-\mathrm{H}(\mathrm{Ar})$ \\
\hline & & & & & 5874 & 5897 & & & $2 v \mathrm{C}-\mathrm{H}_{2}$ \\
\hline & 5788 & 5786 & 5785 & 5785 & 5746 & 5785 & 5785 & 5785 & $2 v_{\mathrm{as}} \mathrm{C}-\mathrm{H}_{2}$ \\
\hline & 5692 & 5698 & 5698 & 5697 & & & 5679 & 5679 & $2 v_{\mathrm{s}} \mathrm{C}-\mathrm{H}_{2}$ \\
\hline \multirow[t]{3}{*}{5253} & 5240 & 5240 & 5249 & 5258 & & 5254 & 5248 & 5253 & $(v+\delta)(\mathrm{OH})_{\mathrm{w}}$ \\
\hline & & & & & 4722 & 4732 & 4734 & 4734 & $3 \delta \mathrm{CH}_{2}=\mathrm{C}$ \\
\hline & & & & & 4641 & 4647 & 4648 & 4648 & $(v \mathrm{CH}+v \mathrm{CC}) \mathrm{Ar}$ \\
\hline \multirow[t]{5}{*}{4528} & 4526 & 4524 & 4524 & 4520 & & 4532 & 4526 & 4522 & $(v+\delta)(\mathrm{OH})_{\mathrm{m}}$ \\
\hline & & & & & 4491 & 4483 & & & $(v \mathrm{CH}+v \mathrm{CC}) \mathrm{Ar}$ \\
\hline & 4467 & 4466 & 4465 & 4446 & & & 4467 & 4450 & $(v+\delta) \mathrm{CH}_{3}-\mathrm{N}$ \\
\hline & 4334 & 4332 & 4331 & 4330 & 4344 & 4340 & 4333 & 4333 & $(v+\delta)_{\mathrm{as}} \mathrm{CH}_{2}$ \\
\hline & 4257 & 4255 & 4253 & 4255 & & & 4259 & 4259 & $(v+\delta) \mathrm{CH}_{3}-\mathrm{N}$ \\
\hline
\end{tabular}

s: simétrico; as: asimétrico 
Trabajos publicados en revistas periódicas

Gamba, M., Olivelli, M., Lázaro-Martínez, J. M., Gaddi, G., Curutchet, G., \& Sánchez, R. M. T. (2017). Thiabendazole adsorption on montmorillonite, octadecyltrimethylammonium-and Acremonium sp.-loaded products and their copper complexes. Chemical Engineering Journal, 320, 11-21.

Melánová, K., Kováŕ, P., Gamba, M., Pospíšil, M., Beneš, L., Zima, V., \& Knotek, P. (2017). Structural Arrangement of 4-[4-(Dimethylamino) phenylazo] pyridine Push-Pull Molecules in Acidic Layered Hosts Solved by Experimental and Calculation Methods. European Journal of Inorganic Chemistry, (1), 115-123.

Gamba, M., Kovář, P., Pospíšil, M., \& Sánchez, R. M. T. (2017). Insight into thiabendazole interaction with montmorillonite and organically modified montmorillonites. Applied Clay Science, 137, 59-68.

Flores, F. M., Gamba, M., Sánchez, R. T., Brendlé, E., \& Brendlé, J. (2016). Microelectrophoresis and inverse gas chromatography as tools to study the surface interactions between a fluorinated fungicide and raw or organically modified Patagonian montmorillonite. Applied Clay Science, 134, 83-88.

Gamba, M., Flores, F. M., Madejová, J., \& Torres Sánchez, R. M. (2015). Comparison of imazalil removal onto montmorillonite and nanomontmorillonite and adsorption surface sites involved: an approach for agricultural wastewater treatment. Industrial \& Engineering Chemistry Research, 54(5), 1529-1538. 